\title{
Tele-Substitution Reactions in the Synthesis of a Promising Class of Antimalarials
}

Marat Korsik, Edwin Tse, David Smith, William Lewis, Peter J. Rutledge, Matthew Todd

Submitted date: 05/05/2020 - Posted date: 06/05/2020

Licence: CC BY-NC-ND 4.0

Citation information: Korsik, Marat; Tse, Edwin; Smith, David; Lewis, William; Rutledge, Peter J.; Todd, Matthew (2020): Tele-Substitution Reactions in the Synthesis of a Promising Class of Antimalarials.

ChemRxiv. Preprint. https://doi.org/10.26434/chemrxiv.12212927.v1

We have discovered and studied a telesubstitution reaction in a biologically important heterocyclic ring system. Conditions that favour the tele-substitution pathway were identified: the use of increased equivalents of the nucleophile or decreased equivalents of base, or the use of softer nucleophiles, less polar solvents and larger halogens on the electrophile. Using results from X-ray crystallography and isotope labelling experiments a mechanism for this unusual transformation is proposed. We focused on this triazolopyrazine as it is the core structure of the in vivo active anti-plasmodium compounds of Series 4 of the Open Source Malaria consortium. Archive of the electronic laboratory notebook with the description of all conducted experiments and raw NMR data could be accessed via following link https://ses.library.usyd.edu.au/handle/2123/21890 . For navigation between entries of laboratory notebook please use file "Strings for compounds in the article.pdf" that works as a reference between article codes and notebook codes, also this file contain SMILES for these compounds.

File list (5)

Tele-substitution_article_Revised.pdf (2.55 MiB)

view on ChemRxiv - download file

Tele-substitution_SI_Revised.pdf (16.05 MiB) view on ChemRxiv • download file

Strings for compounds in the article.pdf ( $49.73 \mathrm{KiB})$ view on ChemRxiv • download file KinomeScan_data_SI.pdf (482.21 KiB) view on ChemRxiv - download file view on ChemRxiv • download file 


\title{
Tele-substitution Reactions in the Synthesis of a Promising Class of Antimalarials
}

\author{
Marat Korsik, ${ }^{\dagger}$ Edwin G. Tse, ${ }^{\dagger} \ddagger$ David G. Smith, "William Lewis, ${ }^{\dagger}$ Peter J. \\ Rutledge, ${ }^{*, \dagger}$ and Matthew H. Todd $*, \ddagger$ \\ $\dagger$ School of Chemistry, The University of Sydney, Sydney, NSW 2006, Australia \\ $\ddagger$ School of Pharmacy, University College London, 29-39 Brunswick Square, London WC1N \\ $1 A X, U . K$. \\ \School of Health and Life Sciences, Federation University, Gippsland Campus, VIC 3842, \\ Australia \\ E-mail: peter.rutledge@sydney.edu.au; matthew.todd@ucl.ac.uk \\ Phone: +61 29351 5020; +44 2077535568
}

\section{Abstract}

We have discovered and studied a telesubstitution reaction in a biologically important heterocyclic ring system. Conditions that favour the tele-substitution pathway were identified: the use of increased equivalents of the nucleophile or decreased equivalents of base, or the use of softer nucleophiles, less polar solvents and larger halogens on the electrophile. Using results from X-ray crystallography and isotope labelling experiments a mechanism for this unusual transformation is proposed. We focused on this triazolopyrazine as it is the core structure of the in vivo active anti-plasmodium compounds of Series 4 of the Open Source Malaria consortium.

\section{Introduction}

Nucleophilic substitution is a widely employed method for functionalising electron deficient aromatic systems. Most commonly, a halide or other leaving group is simply displaced by an incoming nucleophile, known as direct or ipsosubstitution. ${ }^{1}$ Under some circumstances however, a leaving group may be displaced from an aromatic system by a nucleophile entering at a different position on the ring, for example at the carbon adjacent to the leaving group (cine-substitution $^{2}$ ) or even further away (telesubstitution, ${ }^{3}$ Figure 1A). We report here our discovery, and mechanistic studies, of a telesubstitution reaction in a $[1,2,4]$ triazolo $[4,3-$ a]pyrazine system, which is at the core of a series of molecules with significant potential for the future treatment of malaria. ${ }^{4}$

The first example of a tele-substitution reaction was reported in 1930 (Figure 1B). ${ }^{5}$ In this case, the reaction of 2 -(chloromethyl)furan (1) with $\mathrm{NaCN}$ resulted in the attachment of the nitrile group not in place of the chlorine atom, but instead distant from the expected electrophilic site on the opposite side of the furan ring (2). Other examples of tele-substitution reactions have since been reported for a variety of aromatic systems ranging from simple pyrazine rings ${ }^{6}$ (Figure 1C) to more complex triazolopyrazine ring systems ${ }^{7,8}$ (Figures $1 \mathrm{D}$ and $1 \mathrm{E}$ ), the latter of which are of particular relevance to the present work. Despite these and other reports, ${ }^{9-12}$ tele-substitution reactions are not well understood; they remain hard to predict and appear to be strongly substrate dependent. Interestingly, many of the known examples of tele-substitution involve aza-aromatic ring systems which are common in medicinal chemistry 
A<smiles>[X]c1ccccc1O</smiles>

B

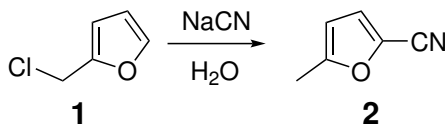

C

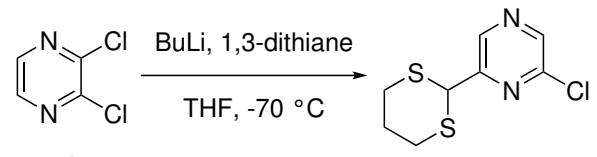

3

4

D<smiles>Brc1cncc2ncnn12</smiles><smiles>CCOc1nccn2ncnc12</smiles>

E

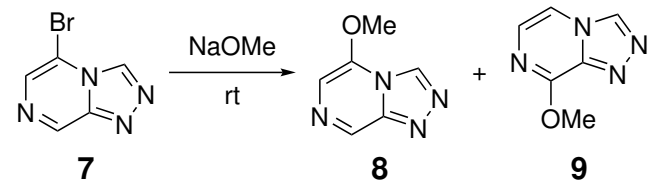

Figure 1: A) Possible positions for nucleophilic aromatic substitution of X. B) First reported case of a tele-substitution reaction in 1930. Further reports of tele-substitution in C) pyrazine, D) $[1,2,4]$ triazolo[1,5-a]pyrazine and E) $[1,2,4]$ triazolo[4,3-a]pyrazine ring systems.

and drug discovery campaigns. Given the isomeric nature of the ipso- and tele-substituted products, and the sometimes cursory level of characterisation in medicinal chemistry articles (where compound identity may be demonstrated using only a ${ }^{1} \mathrm{H}$ NMR spectrum and an LCMS trace) it is important, as we have discovered, to be aware of the possibility of this underappreciated reaction in order to avoid drawing conclusions from erroneous SAR data.

Here, we illustrate this with our studies on the tele-substitution reactions of the $[1,2,4]$ triazolo[4,3-a]pyrazine (hereafter referred to as 'triazolopyrazine') heterocyclic system. These nitrogen-rich, electron-deficient heterocycles are important building blocks for the development of new medicines and have shown a wide variety of biological activities (Figure 2). We have an interest in this motif because it forms the core of Series 4 of the Open Source Malaria (OSM) consortium, ${ }^{13}$ represented here by compound $\mathbf{1 0}$ which possesses in vitro ${ }^{14}$ $\left(\mathrm{IC}_{50}=38 \mathrm{nM}\right)$ and in vivo ${ }^{15}$ antimalarial activity. Compound $\mathbf{1 1}$ has been reported to have nanomolar potency as an inhibitor of the kidney urea transporter UT-A1. ${ }^{16}$ Compound
12 was recently patented in 2016 as a renal outer medullary potassium channel (ROMK) inhibitor. ${ }^{17}$ Sitagliptin (13) was approved by the FDA in 2006 as an antidiabetic drug (dipeptidyl peptidase (DPP)-IV inhibitor). ${ }^{18}$ Compound 14 is a lead molecule $\left(\mathrm{IC}_{50}<100 \mathrm{nM}\right)$, that acts as an inhibitor of bromodomain and extra-terminal motif (BET) proteins for cancer treatment. ${ }^{19}$ Compound $\mathbf{1 5}$ is patented as an $N$-methyl-D-aspartate subtype 2B (NMDAR2B) receptor antagonist. ${ }^{20}$

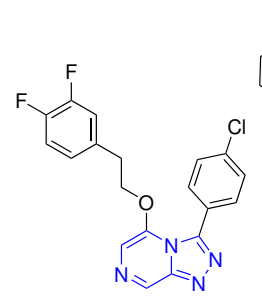

10

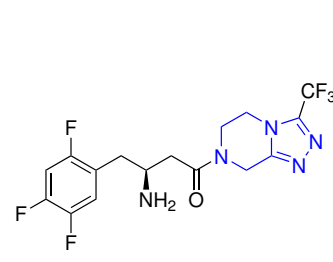

Sitagliptin, 13

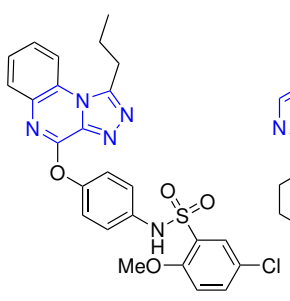

11

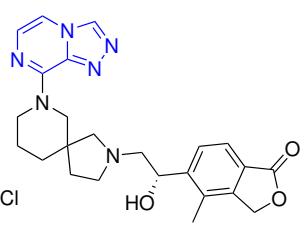

12
Figure 2: Examples of bioactive molecules that include a triazolopyrazine motif or close derivative. 10 is an example compound from OSM Series 4; $\mathbf{1 1}$ is an inhibitor of the UT-A1 transporter; 12 is a ROMK inhibitor; Sitagliptin (13) is an FDA approved antidiabetic drug; $\mathbf{1 4}$ is a BET inhibitor with potential in cancer treatment; $\mathbf{1 5}$ is an NMDAR2B receptor antagonist.

\section{Results and discussion}

The synthesis of members of OSM Series 4 relies on a routine $S_{N} A r$ reaction involving the nucleophilic displacement of a chlorine atom from a triazolopyrazine core (e.g. 16). When the synthesis of thioether analogue $\mathbf{1 7}$ was attempted using the standard conditions for this reaction (Figure $3 \mathrm{~A}$ ), in addition to this expected product, a compound with a significantly lower TLC retention factor was observed and isolated. This was later identified to be 


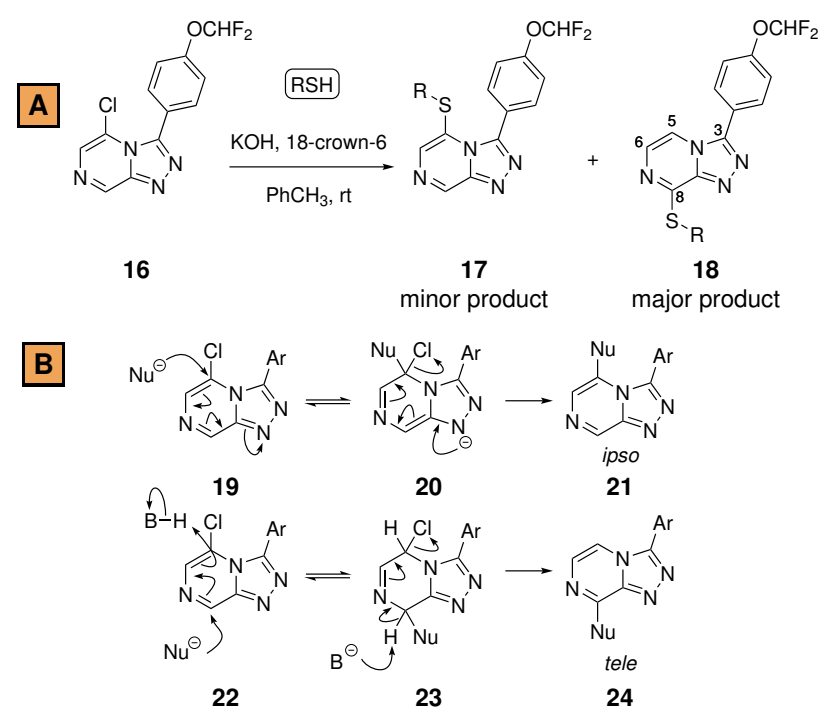

Figure 3: A) Reaction used to make an OSM Series 4 compound $\mathbf{1 7}$, and its tele-substituted isomer 18. B) Proposed mechanisms for ipso- and tele-substitution. ${ }^{21} \mathrm{R}=\mathrm{CH}_{2} \mathrm{CH}_{2} \mathrm{Ph}$.

the tele-substituted isomer $\mathbf{1 8}$. Since the 8isomer $\mathbf{1 8}$ is a main product that was formed in $83 \%$ yield and due to the similarity of the ${ }^{1} \mathrm{H}$ NMR spectra of these two isomers (Figure 4), the tele-substituted isomer $\mathbf{1 8}$ was initially misassigned as the desired product 17. After the reaction had been repeated and examined more thoroughly compound $\mathbf{1 7}$ was successfully isolated as a minor product with $8 \%$ yield. The diagnostic spectroscopic difference between these isomers lies in the peaks arising from the hydrogen atoms at positions 5 and 8 on the triazolopyrazine ring; the correspondence between the NMR spectra and the structures was confirmed using X-ray crystallography (vide infra) and deuteration experiments. In a medicinal chemistry context, this spectroscopic similarity is a hazard for the understanding of structure activity relationships: the original evaluation of this synthetic product had concluded that 17 was inactive $\left(\mathrm{IC}_{50}>10 \mu \mathrm{M}\right)$ in a malaria parasite killing assay (in vitro against $P$. falciparum 3D7 strain), when in fact it was $\mathbf{1 8}$ that had been evaluated in its place. Compound $\mathbf{1 7}$ was later tested and found to have reasonable potency $\left(\mathrm{IC}_{50}=1.04 \mu \mathrm{M}\right)$.

According to the generally accepted ipsosubstitution reaction mechanism, the first step is nucleophile attack on the carbon atom to which halogen is attached (19, Figure 3B). The resulting intermediate (20) expels chloride, leading to the ipso-substituted product (21). On the other hand, a plausible mechanism for the tele-substitution reaction could involve the initial attack of the nucleophile at the 8-position (22, Figure 3B), followed by loss of the 8-position proton as part of the elimination of the chloride (23). Since mechanistic studies on tele-substitution reactions are scarce in the literature, we sought better understanding of the process operating in this case.

To better define the scope of tele-substitution in this triazolopyrazine system, 8- and 6halogenated variants of the triazolopyrazine core were synthesised from the corresponding dihalopyrazines following literature procedures $^{22}$ and subjected to the same reaction conditions as the original 5-chloro triazolopyrazine. The 8-halogenated cores (25-27, Figure 5A) reacted to give the expected ipso-substituted products only (28-36), while the 6-halogenated analogues (37 and $\mathbf{3 8}$, Figure 5B) resulted only in degradation of starting material without formation of any substituted product. While there is limited literature precedence, dihalopyrazines (e.g. 39-41, Figure 5C) have been shown to give exclusively ipso-substituted products (4244 respectively). With these experiments showing that the tele-substitution reaction is observed only with the 5-halogenated cores (Figure 3A), the following mechanistic discussion will focus on that system.

Factors influencing ipso- vs. telesubstitution.

A) Influence of triazolopyrazine structure and nucleophile. ${ }^{\mathrm{a}}$ The nature of the nucleophile plays a crucial role in the outcome of the reaction (Table 1). When compared to reactions with alcohols, the use of more nucleophilic amines and thiols led to significantly more tele-substituted products (Entries 1-6, 1217 and 21-26). This trend may explain why tele-substituted isomers were apparently not seen in the literature synthesis of related structures $^{23}$ in which the incoming nucleophile was restricted to alcohols.

${ }^{a}$ When the conditions employed with alcohols and thiols (KOH, 18-crown-6) were used with amine nucle- 


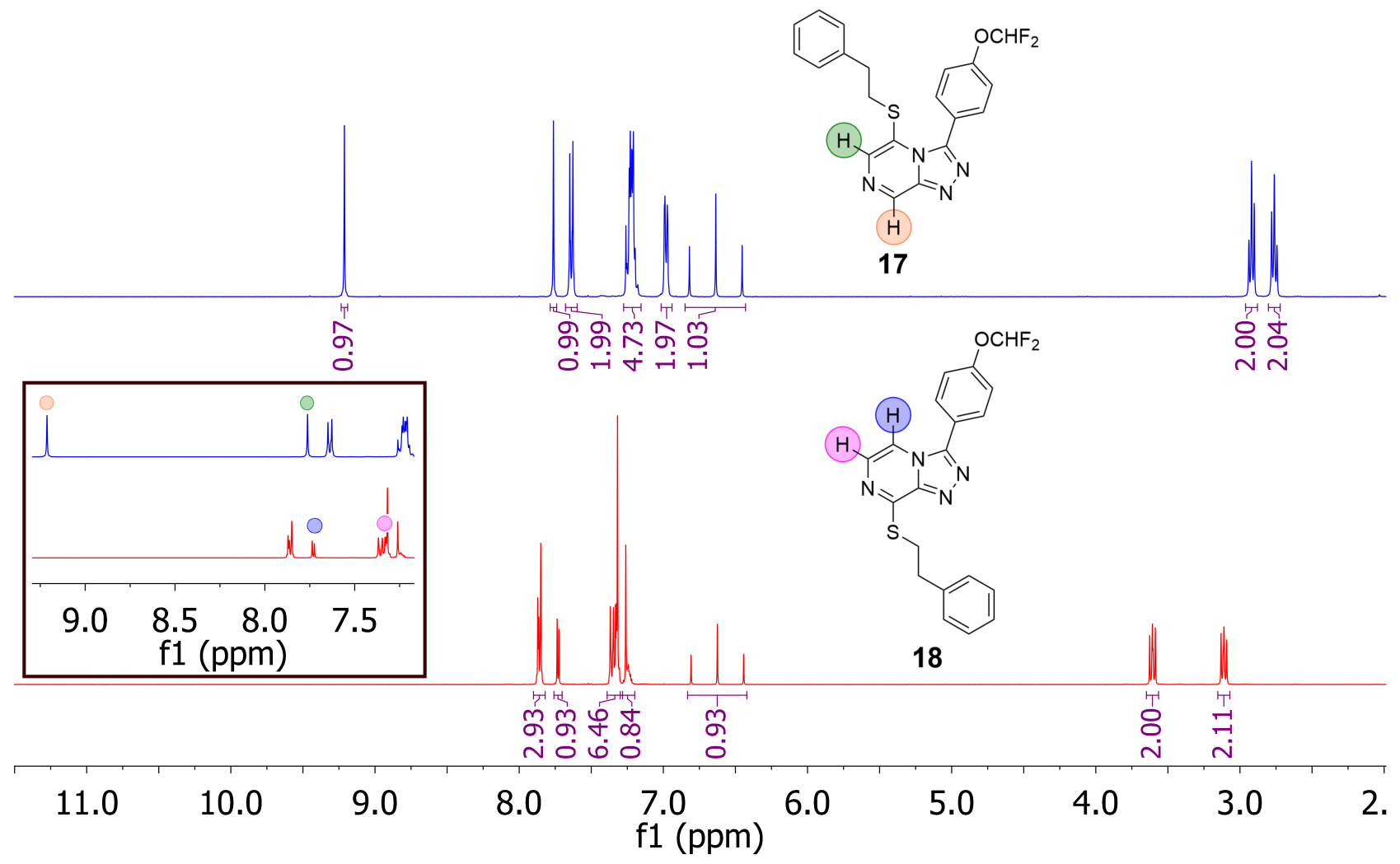

Figure 4: ${ }^{1} \mathrm{H}$ NMR spectra of 17 and 18 in $\mathrm{CDCl}_{3}$. The hydrogen atoms on the pyrazine ring for the 5 -substituted isomers (highlighted in green and orange in 17) give rise to sharp singlets $(\delta \sim 7.5$ and $\sim 9.0 \mathrm{ppm}$ ), while those in the 8-substituted isomers (highlighted in pink and blue in 18) give well-defined doublets $(\delta \sim 7.2-7.7 \mathrm{ppm}, J=4.6 \mathrm{~Hz})$.

The nature of the leaving halogen also influences the outcome, with tele-substitution favoured in the order $\mathrm{I}>\mathrm{Br}>\mathrm{Cl}$ (compare ratio in Entries 4, 15 and 24).

In cases where a larger substituent is in position 3 of the triazolopyrazine core (e.g. a (4$\mathrm{OMe}) \mathrm{Ph}$ group compared to a hydrogen atom), and the leaving halogen is either a Br or I atom, the distribution of ipso- to tele-substituted products is favoured towards the latter (compare Entries 12 and 15 or 21 and 24). Similar experiments in which the leaving halogen is a $\mathrm{Cl}$ atom show little to no change in distribution of products (compare Entries 1 and 4). Further investigation of the substituent at the

ophiles, the reaction progress was comparatively slow so the base was replaced with silica, which gave better conversion; for convenience the rate was made comparable to those seen with the other nucleophiles by raising the reaction temperature, as the reaction at room temperature was not complete after 2 weeks. 3-position led to the conclusion that bulkiness does not affect the reaction (i.e. substitution with (4-OMe) $\mathrm{Ph}$ is comparable to that of the larger $(3,5-t \mathrm{Bu}) \mathrm{Ph}$ or 9-anthracene; Entries 4, 10 and 11 respectively).

Substrates with electron donating (EDG) and electron withdrawing (EWG) groups on the phenyl ring at the 3-position of the core were studied in order to evaluate the influence of electronic effects on the distribution of products. Experiments on bromotriazolopyrazines showed that EDGs tend to promote the tele-substitution pathway of the reaction, while EWGs lead to ipso-products only (Entries 15 and 18-20). Interestingly, chloro-triazolopyrazines do not follow this pattern and show no dependence on the electronic effects from the substituent in the 3-position (Entries 4, 7, 8 and 9).

From the experiments summarised in Table 1 , two gave surprising results. The reaction be- 
Table 1: Influence of triazolopyrazine structure, leaving halogen $\mathrm{X}$ and nucleophile on the reaction outcome. Conditions: ${ }^{\mathrm{a}} \mathrm{KOH}, 18$-crown-6, toluene, room temperature; ${ }^{\mathrm{b}}$ silica, toluene, reflux. ${ }^{\mathrm{c}}$ Dehalogenation by-product 49 was isolated as well in $74 \%$ yield. ${ }^{\mathrm{d}}$ Dehalogenation by-product 49 was isolated in $11 \%$ yield along with ring opening product 50 in $17 \%$ yield (refer to Figure 6 for details). $\mathrm{R}_{1}=\mathrm{CH}_{2} \mathrm{CH}_{2} \mathrm{Ph}$. ND: not determined.
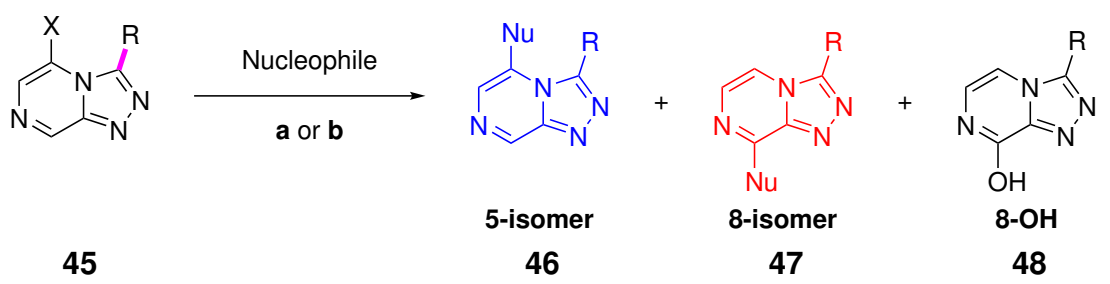

\begin{tabular}{|c|c|c|c|c|c|c|c|}
\hline Entry & $\mathrm{X}$ & $\mathrm{R}$ & 45 & Nucleophile & 46 yield $[\%]$ & 47 yield $[\%]$ & 48 yield $[\%]$ \\
\hline 1 & $\mathrm{Cl}$ & $\mathrm{H}$ & $45 a$ & $\mathrm{R}_{1} \mathrm{OH}$ & $46 \mathbf{a}, 75$ & ND & - \\
\hline 2 & $\mathrm{Cl}$ & $\mathrm{H}$ & $45 a$ & $\mathrm{R}_{1} \mathrm{SH}$ & $46 \mathrm{~b}, 51$ & ND & - \\
\hline 3 & $\mathrm{Cl}$ & $\mathrm{H}$ & $45 a$ & $\mathrm{R}_{1} \mathrm{NH}_{2}^{\mathrm{b}}$ & ND & 30,65 & - \\
\hline 4 & $\mathrm{Cl}$ & $(4-\mathrm{OMe}) \mathrm{Ph}$ & $45 \mathrm{~b}$ & $\mathrm{R}_{1} \mathrm{OH}$ & $46 c, 70$ & $47 a, 3$ & - \\
\hline 5 & $\mathrm{Cl}$ & $(4-\mathrm{OMe}) \mathrm{Ph}$ & $45 \mathrm{~b}$ & $\mathrm{R}_{1} \mathrm{SH}$ & 46d, 8 & $47 \mathrm{~b}, 83$ & - \\
\hline 6 & $\mathrm{Cl}$ & $(4-\mathrm{OMe}) \mathrm{Ph}$ & $45 \mathrm{~b}$ & $\mathrm{R}_{1} \mathrm{NH}_{2}{ }^{\mathrm{b}}$ & ND & $47 c, 89$ & - \\
\hline 7 & $\mathrm{Cl}$ & $\left(4-\mathrm{NO}_{2}\right) \mathrm{Ph}$ & $45 \mathrm{c}$ & $\mathrm{R}_{1} \mathrm{OH}$ & 46e, 77 & 34,2 & - \\
\hline 8 & $\mathrm{Cl}$ & $(2-\mathrm{OMe}) \mathrm{Ph}$ & $45 d$ & $\mathrm{R}_{1} \mathrm{OH}$ & 46f, 58 & ND & - \\
\hline 9 & $\mathrm{Cl}$ & $\left(2-\mathrm{NO}_{2}\right) \mathrm{Ph}$ & $45 \mathrm{e}$ & $\mathrm{R}_{1} \mathrm{OH}$ & $46 \mathrm{~g}, 65$ & ND & - \\
\hline 10 & $\mathrm{Cl}$ & $(3,5-t \mathrm{Bu}) \mathrm{Ph}$ & $45 \mathrm{f}$ & $\mathrm{R}_{1} \mathrm{OH}$ & 46h, 82 & $47 \mathrm{~d}, 3$ & - \\
\hline 11 & $\mathrm{Cl}$ & 9-anthracenyl & $45 \mathrm{~g}$ & $\mathrm{R}_{1} \mathrm{OH}$ & $\mathbf{4 6} \mathbf{i}, 66$ & $47 \mathbf{e}, 2$ & - \\
\hline 12 & $\mathrm{Br}$ & $\mathrm{H}$ & $45 \mathrm{~h}$ & $\mathrm{R}_{1} \mathrm{OH}$ & $\mathbf{4 6 a}, 66$ & ND & - \\
\hline 13 & $\mathrm{Br}$ & $\mathrm{H}$ & $45 \mathrm{~h}$ & $\mathrm{R}_{1} \mathrm{SH}$ & $46 \mathrm{~b}, 34$ & $\mathbf{2 9}, 17$ & - \\
\hline 14 & $\mathrm{Br}$ & $\mathrm{H}$ & $45 \mathrm{~h}$ & $\mathrm{R}_{1} \mathrm{NH}_{2}^{\mathrm{b}}$ & ND & $\mathbf{3 0}, 72$ & - \\
\hline 15 & $\mathrm{Br}$ & $(4-\mathrm{OMe}) \mathrm{Ph}$ & $45 \mathrm{i}$ & $\mathrm{R}_{1} \mathrm{OH}$ & $\mathbf{4 6 c}, 32$ & $47 \mathbf{a}, 10$ & $48 \mathbf{a}, 20$ \\
\hline 16 & $\mathrm{Br}$ & $(4-\mathrm{OMe}) \mathrm{Ph}$ & $45 \mathrm{i}$ & $\mathrm{R}_{1} \mathrm{SH}$ & ND & $47 \mathrm{~b}, 93$ & - \\
\hline 17 & $\mathrm{Br}$ & $(4-\mathrm{OMe}) \mathrm{Ph}$ & $45 \mathrm{i}$ & $\mathrm{R}_{1} \mathrm{NH}_{2}{ }^{\mathrm{b}}$ & ND & $47 c, 66$ & - \\
\hline 18 & $\mathrm{Br}$ & $\left(4-\mathrm{NO}_{2}\right) \mathrm{Ph}$ & $45 \mathrm{j}$ & $\mathrm{R}_{1} \mathrm{OH}$ & $\mathbf{4 6 e}, 60$ & ND & $48 \mathrm{~b}, 30$ \\
\hline 19 & $\mathrm{Br}$ & $(2-\mathrm{OMe}) \mathrm{Ph}$ & $45 \mathrm{k}$ & $\mathrm{R}_{1} \mathrm{OH}$ & $\mathbf{4 6 f}, 26$ & $47 f, 9$ & $\mathbf{4 8 c}, 49$ \\
\hline 20 & $\mathrm{Br}$ & $\left(2-\mathrm{NO}_{2}\right) \mathrm{Ph}$ & 451 & $\mathrm{R}_{1} \mathrm{OH}$ & $46 \mathrm{~g}, 76$ & ND & $\mathbf{4 8 d}, 12$ \\
\hline 21 & I & $\mathrm{H}$ & $45 \mathrm{~m}$ & $\mathrm{R}_{1} \mathrm{OH}$ & $\mathbf{4 6 a}, 34$ & ND & - \\
\hline 22 & I & $\mathrm{H}$ & $45 \mathrm{~m}$ & $\mathrm{R}_{1} \mathrm{SH}$ & $46 \mathrm{~b}, 7$ & 29,7 & - \\
\hline 23 & $\mathrm{I}$ & $\mathrm{H}$ & $45 \mathrm{~m}$ & $\mathrm{R}_{1} \mathrm{NH}_{2}{ }^{\mathrm{b}}$ & ND & $\mathbf{3 0}, 40$ & - \\
\hline 24 & I & $(4-\mathrm{OMe}) \mathrm{Ph}$ & $45 n$ & $\mathrm{R}_{1} \mathrm{OH}$ & $\mathbf{4 6 c}, 23$ & $47 \mathbf{a}, 53$ & - \\
\hline $25^{\mathrm{c}}$ & I & $(4-\mathrm{OMe}) \mathrm{Ph}$ & $45 n$ & $\mathrm{R}_{1} \mathrm{SH}$ & ND & $47 \mathrm{~b}, 13$ & - \\
\hline $26^{\mathrm{d}}$ & I & $(4-\mathrm{OMe}) \mathrm{Ph}$ & $45 n$ & $\mathrm{R}_{1} \mathrm{NH}_{2}{ }^{\mathrm{b}}$ & ND & $47 c, 58$ & - \\
\hline
\end{tabular}

tween the iodo-triazolopyrazine core $45 \mathrm{n}$ and the thiol nucleophile (Entry 25) in addition to the 8 -substituted compound $\mathbf{4 7 b}$, isolated in $13 \%$ yield, gave dehalogenated product 49 in $74 \%$ yield. This product was not observed for any other reaction substrates bearing a chlorine or bromine atom. This type of the dehalogenation reaction has not previously been reported in the literature. The other unexpected product was isolated from the reaction between the iodo-triazolopyrazine core $\mathbf{4 5 n}$ and the amine nucleophile (Entry 26). In addition to the isolation of the major tele-substituted isomer $47 \mathbf{c}$ and dehalogenation product 49, a minor byproduct was obtained in $17 \%$ yield, the structure was determined by single crystal X-ray 


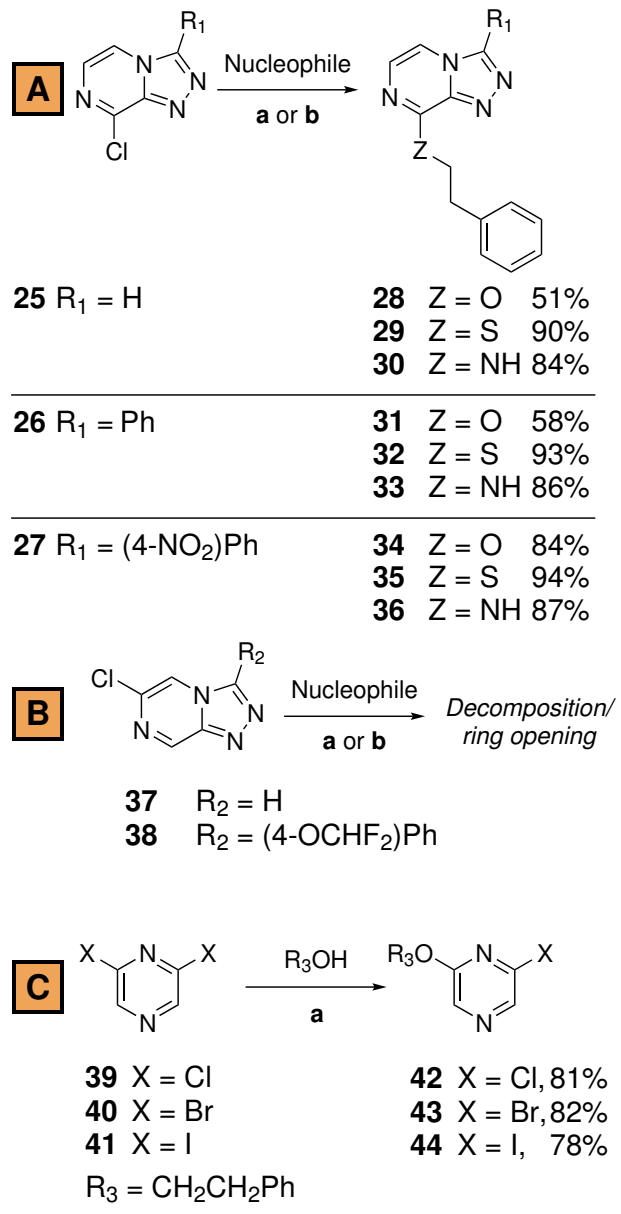

Figure 5: Reactions of halogenated triazolopyrazine isomers and pyrazines. A) 8-Isomer; B) 6Isomer; C) Pyrazine; Conditions: ${ }^{a} \mathrm{KOH}, 18$-crown6 , toluene, room temperature (reactions involve measuring small amounts of hygroscopic $\mathrm{KOH}$, which can contribute to reproducibility challenges, thus experiments were performed in duplicate and are reported as average values); ${ }^{\mathrm{b}}$ silica, toluene, reflux (more details in Table 1).

diffraction to be based on a 5-(1H-imidazol-2yl)-1H-1,2,4-triazole core instead of triazolopyrazine (50, Figure 6). It is possible that compound 50 could be formed via initial nucleophile attack at the 8-position of the pyrazine ring (51), followed by the pyrazine ring opening (52) and rearrangement (53) leading to 50. While the analogous reaction utilising the chlorine-substituted triazolopyrazine (Entry 6) did not lead to this rearranged product, it was formed in trace amounts when the bromosubstituted triazolopyrazine was employed (Entry 17). This trend may either be due to a suboptimal bond geometry (i.e. pseudo-equatorial
I atom) arising from the larger halogen atom or from a better match of orbital energies for elimination (in the case of the chlorine leaving group).

B) Influence of solvent. With the reaction between 45i and the alcohol nucleophile (Table 1, Entry 1) giving significant quantities of both isomers, this was used as the model reaction to investigate further the influence of solvent on the reaction outcome (Table 2). A screen of aprotic solvents clearly showed that solvents with higher dielectric constants lead to less telesubstitution and also lowers the overall yield of the reaction. Protic solvents are inherently unsuitable for this reaction as they can easily themselves react with the halogenated triazolopyrazine. This was demonstrated when water was used as the solvent, giving the product $48 \mathbf{a}$ in $94 \%$ yield, by result of tele-substitution with $\mathrm{H}_{2} \mathrm{O}$.

C) Influence of excess alcohol and base. By using the same model reaction above, the effect of alcohol and base equivalents was investigated. It was found that the use of an excess of nucleophile resulted in a shift of the reaction outcome drastically towards the formation of the 8-isomer (47a, Figure 7A). These observations suggest that the use of a softer nucleophile (here one in which the anion is surrounded by a "solvent shell" of $\mathrm{OH}$ bonds arising from excess nucleophile) leads to greater formation of the 8-isomer. Similarly, when fewer equivalents of base were used, a higher proportion of telesubstitution was again observed (Figure 7B).

D) Influence of water and temperature. In order to evaluate the impact of the level of water present on tele-substitution, the reaction between 45a (unsubstituted on the triazole ring) and piperidine was conducted in toluene with various levels of water, as well as in water itself $\left(\mathrm{H}_{2} \mathrm{O}\right.$ and $\left.\mathrm{D}_{2} \mathrm{O}\right)$. The isolated yields of the 5- (55) and 8-isomer (56) were identical for experiments in both wet and dry toluene (Table 3, Entries 1 and 3). At room temperature the reaction took 14 days to complete (Entry 2), but the outcome was comparable to that when heating under reflux conditions. When molecular sieves were included in the reaction mixture (using dried toluene) the ratio of products 


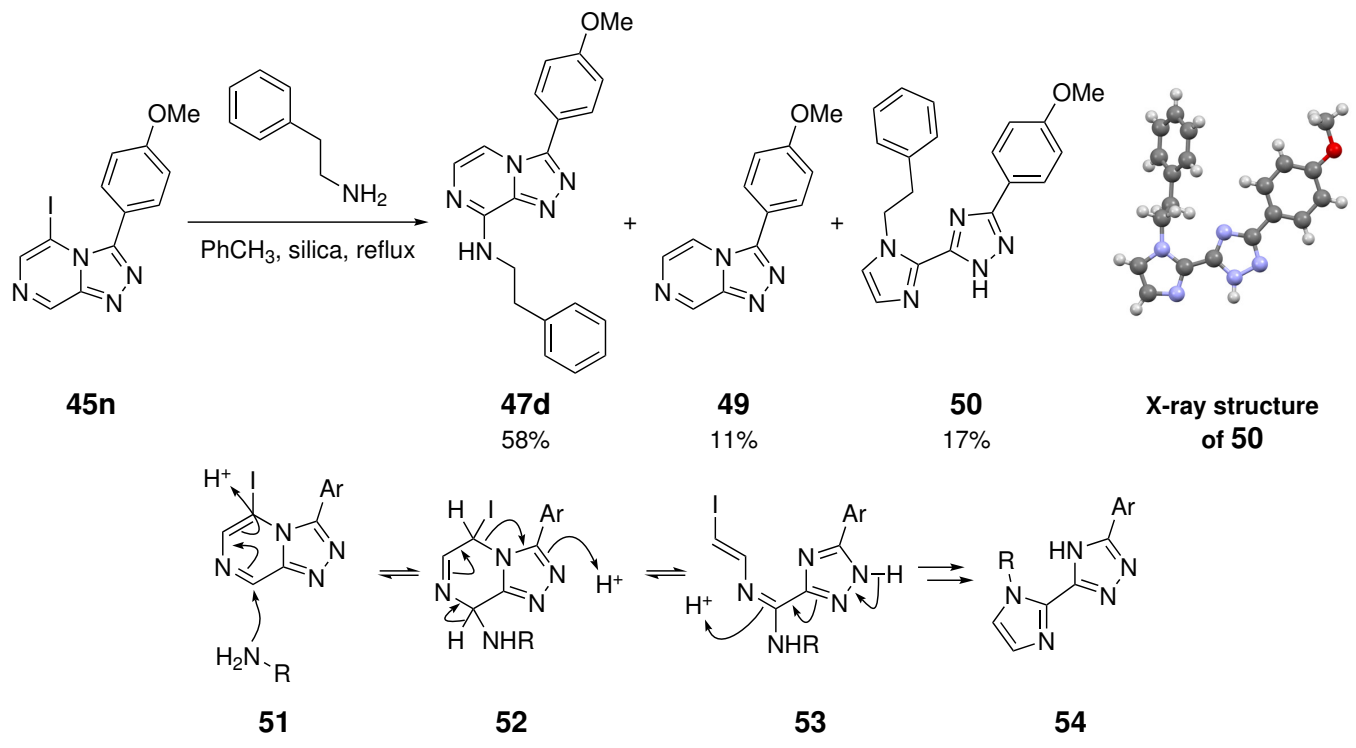

Figure 6: Unexpected product $\mathbf{5 0}$ of ring opening and rearrangement from the reaction of iodotriazolopyrazine and an amine nucleophlie, with an X-ray structure and proposed mechanism for this product (see SI for more details, Figure S2.

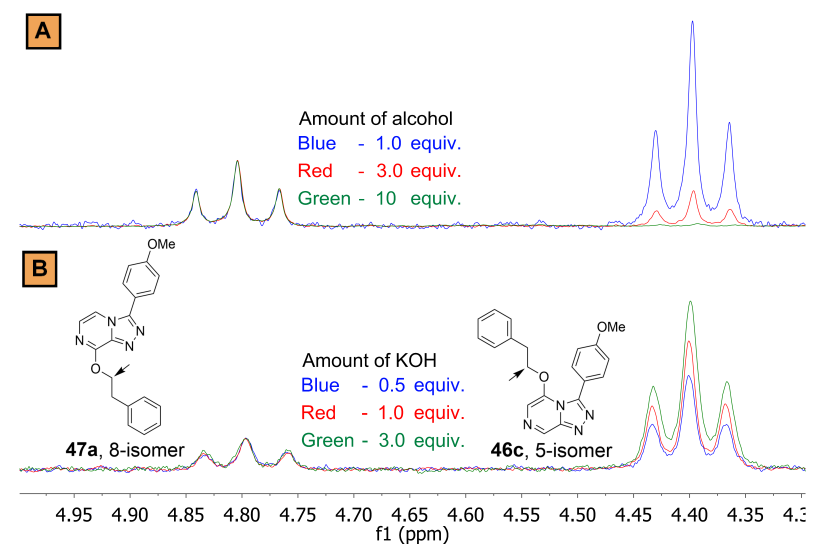

Figure 7: Crude ${ }^{1} \mathrm{H}$ NMR comparison of the reaction outcome depending on A) amount of alcohol; B) amount of base. Structures of isomers placed next to corresponding signals from $\mathrm{CH}_{2}$ groups which are indicated by arrow.

changed, though it is possible that this could arise from catalytic activity at the zeolite surface itself (Entry 4). ${ }^{24,25}$ Performing the reaction in $\mathrm{H}_{2} \mathrm{O}$ (Entry 5) gave a comparable result to that in wet toluene. This is counter to the example where the alcohol nucelophile was out-competed by the solvent water to give the tele-substitution product (vide supra). It could be concluded that the presence of water in the solvent and the reaction temperature do not al- ter the distribution of products in the studied reaction.

E) Isotope labeling experiments. Following the observation that no hydroxy-substituted product was identified in the reaction between the halogenated triazolopyrazine core $\mathbf{4 5 a}$ and an amine nucleophile in the presence of water, deuteration experiments were peformed to gain insight into the reaction mechanism. This reaction was carried out in $\mathrm{D}_{2} \mathrm{O}$ giving two compounds, 57 and 58 (Figure 8A). The examination of products with ${ }^{1} \mathrm{H}$ NMR and ${ }^{2} \mathrm{H}$ NMR spectroscopy showed incorporation of one $\mathrm{D}$ atom in $\mathbf{5 7}$ and two in 58. Both molecules underwent deuterium exchange of the triazole $\mathrm{H}$ atom. The deuteration of triazole rings has been reported in a handful of cases, ${ }^{26,27}$ but not for the triazolopyrazine system investigated here. In order to prove that deuteration at the 3-position occurs as a parallel reaction to the main substitution, compounds $45 \mathrm{a}$, 55 and 56 were heated under reflux in $\mathrm{D}_{2} \mathrm{O}$ without piperidine to give corresponding monodeuterated products $\mathbf{5 9}, \mathbf{5 7}$ and $\mathbf{6 0}$ respectively (Figure 8B). The second $\mathrm{D}$ atom in 58 was at the 5-position, thus confirming that the proton which takes the place of the leaving group in the tele-substitution reaction comes from the 
Table 2: A) Reaction used to study the influence of solvent; B) Product isolated when $\mathrm{H}_{2} \mathrm{O}$ was employed as a solvent. Results of the reaction in different solvents (reactions performed in duplicate). All solvents were dried over molecular sieves $(3 \AA)$ for $48 \mathrm{~h}$ before application. All reactions proceed to complete consumption of bromo-triazolopyrazine as indicated by TLC. Total yield reported is the sum of both isomers. Product 48 a typically observed to form in $\sim 15 \%$ yield but was not isolated in these reactions. $\mathrm{R}$ $=\mathrm{CH}_{2} \mathrm{CH}_{2} \mathrm{Ph}$.

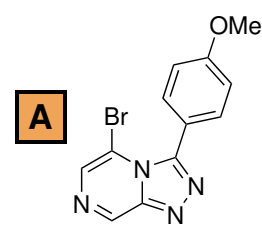

$45 \mathrm{~m}$

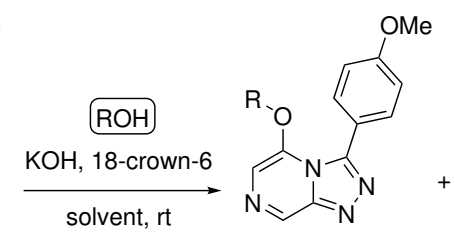

$46 c$

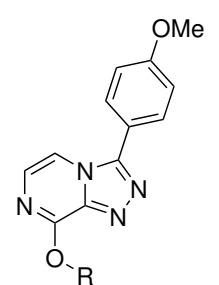

$47 a$

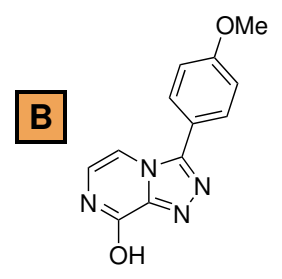

48a

\begin{tabular}{llcccc}
\hline Entry & Solvent & $\begin{array}{c}\text { 46c (5-isomer) } \\
\text { yield [\%] }\end{array}$ & $\begin{array}{c}\text { 47a (8-isomer) } \\
\text { yield [\%] }\end{array}$ & $\begin{array}{c}\text { Total } \\
\text { yield [\%] }\end{array}$ & $\begin{array}{c}\text { Dielectric } \\
\text { constant }\end{array}$ \\
\hline 1 & Cyclohexane & 35 & 24 & 58 & 2.02 \\
2 & Toluene & 31 & 8 & 40 & 2.38 \\
3 & Dioxane & 20 & 9 & 29 & 2.25 \\
4 & THF & 19 & 3 & 22 & 7.58 \\
5 & Acetonitrile & 43 & 3 & 45 & 37.5 \\
6 & DMF & 25 & 2 & 27 & 36.7 \\
\hline
\end{tabular}

solvent and not from the substrate (see the proposed mechanism for 19 in Figure 3B).

Importantly, the amine products 55 and 56 were found to be not interconvertible when each product separately was subjected to the reaction conditions for 3 days, as no conversion of one isomer into another could be detected by TLC. Thus the ratios of products observed in these telesubstitution reactions arise from a kinetic difference rather than one that has a thermodynamic origin.

\section{Biological activity}

As mentioned above, 5-substituted triazolopyrazines (e.g. 17) showed antiplasmodium activity, while an 8-substituted isomer (18) proved to be inactive. Based on the structural similarity of these triazolopyrazines to kinase inhibitors, ${ }^{28}$ we evaluated several compounds in the preliminary KINOMEscan ${ }^{\circledR}$ assay (at 1 $\mu \mathrm{M}$ concentration). The results revealed complementary activity of ipso- and tele-isomers, for example $\mathbf{4 7} \mathrm{b}$ has higher potency against serine/threonine-protein kinase 3 (STK3) com- pared to 46d (Figure 9, see SI for full screening results). Thus the occurrence of this telesubstitution reaction allows the generation of two biologically active compounds with complentary activities from a single reaction.

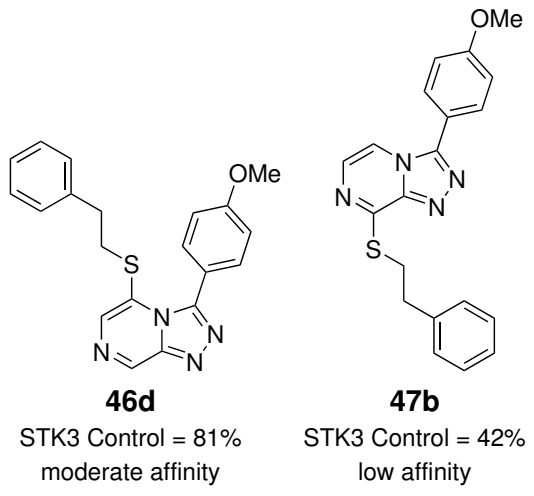

Figure 9: Compounds evaluated in KINOMEscan ${ }^{\circledR}$ assay.

\section{Conclusion}

Tele-substitution reactions are simple to achieve in the triazolopyrazine ring system, and it is important to be aware of the possi- 
Table 3: Results of the reaction with wet and dry solvent. $3 \AA$ molecular sieves were used to dry the toluene. Water levels were measured with a Karl-Fischer titration apparatus immediately before the experiment.

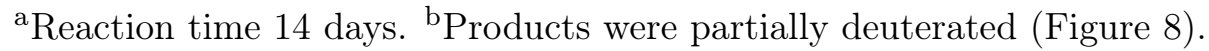



A

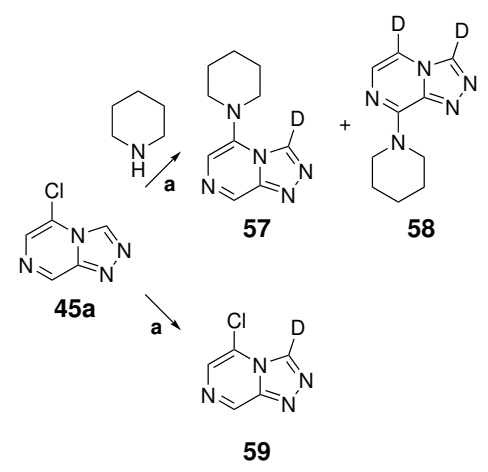

B

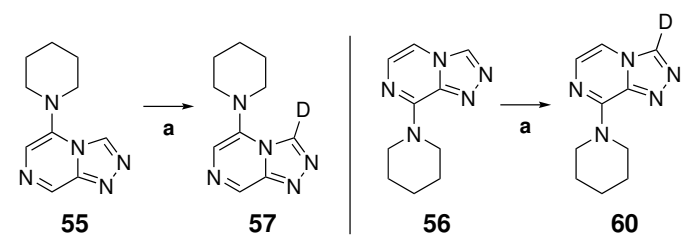

Figure 8: A) Reaction between simplified chlorosubstituted core $\mathbf{4 5 a}$ and piperidine, performed in $\mathrm{D}_{2} \mathrm{O}$ as the solvent; B) Verification that $\mathrm{H} / \mathrm{D}$ exchange on the triazole, but not the pyrazine, is a parallel reaction to the main substitution reaction. ${ }^{a} \mathrm{D}_{2} \mathrm{O}$, heating at reflux.

bility of such isomers forming, given the wide biological relevance of many of these structures. The tele-substitution reaction occurs only in 5-halogenated triazolopyrazine cores, while 8- or 6-halogenated cores tend to give ipso-substitution or degradation respectively. The tele-substitution pathway of the reaction is also made more likely by the use of stronger nu- cleophiles, triazolopyrazines with bulkier halogens and the use of less polar solvents. As concluded from the isotope labeling experiments, the hydrogen atom that takes the place of the halogen derives from solvent and not from substrate. The product ratios arise from a kinetic difference in the reactions rather than a thermodynamic difference in product energies, where, broadly, a combination of hard nucleophile and hard electrophile promotes ipso-substitution while a softer combination promotes tele-substitution.

\section{Experimental}

\subsection{General Procedures}

General Procedure A. Preparation of halogenhydrazinylpyrazines

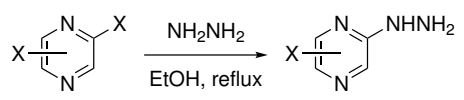

Mono or dihalogenopyrazine (70 mmol, 1 equiv.) was dissolved in ethanol $(100 \mathrm{~mL})$, then hydrazine monohydrate was added (140 mmol, 2 equiv.) and the mixture was heated at reflux overnight. The solvent was removed under reduced pressure. Equal amounts of EtOAc (100 $\mathrm{mL})$ and $\mathrm{H}_{2} \mathrm{O}(100 \mathrm{~mL})$ were added, the EtOAc 
layer was separated and the aqueous layer was washed with EtOAc $(30 \mathrm{~mL} \times 3)$. The combined organic phases were washed with brine $(30 \mathrm{~mL})$, dried over $\mathrm{Na}_{2} \mathrm{SO}_{4}$ and evaporated under reduced pressure to give the desired compound, which was used in the subsequent reaction without further purification.

General Procedure B. Preparation of halogeno-[1,2,4]triazolo[4,3-a]pyrazine

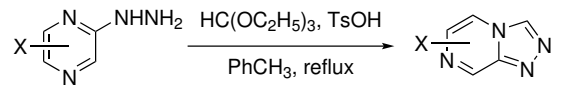

To a suspension of halogen-hydrazinylpyrazine (70.0 mmol, 1.0 equiv.) in toluene $(200 \mathrm{~mL})$ triethyl orthoformate or trimethyl orthoformate (140 mmol, 2.0 equiv.) was added followed by $p$-toluenesulfonic acid monohydrate (14.0 mmol, 0.2 equiv.). The mixture was heated at reflux for $5 \mathrm{~h}$. The solvent was removed under reduced pressure and the residue purified by flash column chromatography (FCC) on silica using a gradient of EtOAc (20\% to $100 \%)$ in hexanes to give the desired product.

General Procedure C. Preparation of halogeno-3-aryl-[1,2,4]triazolo[4,3-a]pyrazine

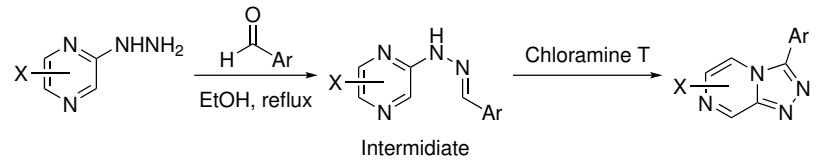

Adopted from the literature procedures. ${ }^{22}$ To a stirred suspension of halogeno-hydrazinylpyrazine $(7.0 \mathrm{mmol}, 1.0$ equiv.) in ethanol $(100 \mathrm{~mL})$ was added aldehyde (7.7 mmol, 1.1 equiv.) and the mixture heated at reflux overnight. After the full consumption of starting material as indicated by TLC, the reaction was cooled in an ice bath and chloramine $\mathrm{T}$ trihydrate (9.1 mmol, 1.3 equiv.) was added portionwise while stirring over $1 \mathrm{~h}$. After consumption of the intermediate was confirmed by TLC, cold $\mathrm{H}_{2} \mathrm{O}(100 \mathrm{~mL})$ was added to the reaction mixture. The solution was stirred for $10 \mathrm{~min}$, then filtered through a sintered glass filter ( $\mathrm{P} 3$ porosity) and washed with $\mathrm{H}_{2} \mathrm{O}(30$ $\mathrm{mL} \times 3)$ followed by $\mathrm{Et}_{2} \mathrm{O}(30 \mathrm{~mL})$. The solid was dried in vacuo to give desired product that was used without further purification.
General Procedure D. Coupling of alcohol or thiol with halogen-heterocycle

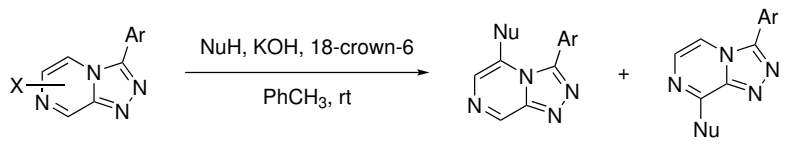

To a suspension of halogen-heterocycle (0.40 mmol, 1 equiv.) in toluene $(10 \mathrm{~mL})$ was added 18-crown-6 (0.032 mmol, 0.08 equiv.) and alcohol or thiol $(0.40 \mathrm{mmol}, 1$ equiv.) followed by $\mathrm{KOH}$ (1.20 mmol, 3.0 equiv.). The reaction mixture stirred for 2-24 h at room temperature. Upon completion as indicated by TLC, the reaction mixture was directly subjected to the purification by FCC on silica and flushed at the beginning with hexanes (in order to wash out toluene from the column) followed by a gradient of EtOAc (30\% to 100\%) in hexanes (unless specified in the compound preparation) to give the desired product.

General Procedure E. Coupling of amine with halogen-heterocycle

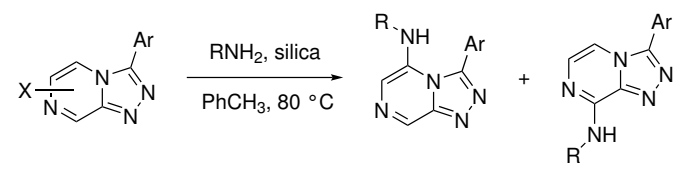

To a suspension of halogen-heterocycle $(0.40$ mmol, 1.0 equiv.) in toluene $(10 \mathrm{~mL})$ was added amine (1.20 mmol, 3.0 equiv.) followed by silica $(0.5 \mathrm{~g})$. The reaction was heated at $80{ }^{\circ} \mathrm{C}$ overnight. Upon completion of the reaction as indicated by TLC, the solvent was evaporated in vacuo and the mixture purified by FCC on silica using a gradient of EtOAc EtOAc (30\% to $100 \%$ ) in hexanes (unless specified in the compound preparation) to give the desired product.

\subsection{Synthesis}

2-Chloro-6-hydrazinylpyrazine (S1). General Procedure A was applied using 2,6dichloropyrazine $(35.0 \mathrm{~g}, 235 \mathrm{mmol})$ to give $\mathbf{S} 1$ as a yellow solid (29.2 g, $202 \mathrm{mmol}, 86 \%)$. mp 137-139 ${ }^{\circ} \mathrm{C}$ (lit. $\left.{ }^{8} 136-139{ }^{\circ} \mathrm{C}\right) .{ }^{1} \mathrm{H}$ NMR (300 $\left.\mathrm{MHz}, \mathrm{DMSO}-d_{6}\right): \delta 8.42(\mathrm{~s}, 1 \mathrm{H}), 8.05(\mathrm{~s}, 1 \mathrm{H})$, $7.70(\mathrm{~s}, 1 \mathrm{H}), 4.37$ (s, 2H). ${ }^{13} \mathrm{C}\left\{{ }^{1} \mathrm{H}\right\}$ NMR (50 $\left.\mathrm{MHz}, \mathrm{DMSO}-d_{6}\right): \delta 157.1,145.7,129.0,128.6$. 
The spectroscopic data and melting point were in agreement with those in the literature. ${ }^{8,29}$

2-Bromo-6-hydrazinylpyrazine (S2). General Procedure A was applied using 2,6dibromopyrazine $(8.09 \mathrm{~g}, 34.0 \mathrm{mmol})$ to give S2 as an orange solid $(5.45 \mathrm{~g}, 28.9 \mathrm{mmol}$, 85\%). mp $142-144{ }^{\circ} \mathrm{C} .{ }^{1} \mathrm{H}$ NMR (500 MHz, $\left.\mathrm{CDCl}_{3}\right): \quad \delta 8.16(\mathrm{~s}, 1 \mathrm{H}), 7.98(\mathrm{~s}, 1 \mathrm{H}), 6.28$ (s, 1H), $3.72(\mathrm{~s}, \quad 2 \mathrm{H}) .{ }^{13} \mathrm{C}\left\{{ }^{1} \mathrm{H}\right\}$ NMR (126 $\left.\mathrm{MHz}, \mathrm{CDCl}_{3}\right): \delta 156.8,138.1,135.4,129.2$. HRMS (ESI/FTICR) $m / z:[\mathrm{M}+\mathrm{H}]^{+}$calcd for $\mathrm{C}_{4} \mathrm{H}_{6}{ }^{79} \mathrm{BrN}_{4}$ 188.97704; found 188.97728.

2-Iodo-6-hydrazinylpyrazine (S3). General Procedure A was applied using 41 (8.37 g, 25.2 mmol) to give $\mathbf{S 3}$ as a yellow solid (4.87 g, 20.7 mmol, 82\%). $\operatorname{mp~} 154-156{ }^{\circ} \mathrm{C} .{ }^{1} \mathrm{H}$ NMR (300 $\left.\mathrm{MHz}, \mathrm{DMSO}-d_{6}\right): \delta 8.31(\mathrm{~s}, 1 \mathrm{H}), 8.05(\mathrm{~s}, 1 \mathrm{H})$, $7.91(\mathrm{~s}, 1 \mathrm{H}), 4.33(\mathrm{~s}, 2 \mathrm{H}) \cdot{ }^{13} \mathrm{C}\left\{{ }^{1} \mathrm{H}\right\} \mathrm{NMR}(75$ MHz, DMSO- $\left.d_{6}\right): \delta 157.8,137.8,128.9,115.9$. HRMS (ESI/FTICR) $m / z:[\mathrm{M}+\mathrm{H}]^{+}$calcd for $\mathrm{C}_{4} \mathrm{H}_{6} \mathrm{IN}_{4} 236.96317$; found 236.96297 .

2-Chloro-3-hydrazinylpyrazine (S4). General Procedure A was applied using 2,3dichloropyrazine (10.2 g, $68.3 \mathrm{mmol})$ to give S4 as a yellow solid (6.61 g, $45.7 \mathrm{mmol}, 67 \%)$. $\operatorname{mp} 156-158{ }^{\circ} \mathrm{C}$ (lit. ${ }^{30} \mathrm{mp} 154{ }^{\circ} \mathrm{C}$ ). ${ }^{1} \mathrm{H}$ NMR $\left(200 \mathrm{MHz}\right.$, DMSO- $\left.d_{6}\right): \delta 8.23(\mathrm{~s}, 1 \mathrm{H}), 8.04(\mathrm{~d}$, $J=2.7 \mathrm{~Hz}, 1 \mathrm{H}), 7.55(\mathrm{~d}, J=2.8 \mathrm{~Hz}, 1 \mathrm{H}), 4.34$ $(\mathrm{s}, 2 \mathrm{H}) \cdot{ }^{13} \mathrm{C}\left\{{ }^{1} \mathrm{H}\right\}$ NMR $\left(50 \mathrm{MHz}, \mathrm{DMSO}-d_{6}\right)$ : $\delta 152.6,140.6,132.6,130.0$. The spectroscopic data and melting point were in agreement with those in the literature. ${ }^{22,30}$

2-Chloro-5-hydrazinylpyrazine (S5). Compound was prepared following literature procedures. ${ }^{31}$ 2,5-Dichloropyrazine (2.00 g, 13.4 mmol, 1.0 equiv.) was added to $\mathrm{H}_{2} \mathrm{O}$ (12.5 $\mathrm{mL}$ ) followed by $28 \%$ aq. ammonia solution (2.63 mL, 38.9 mmol, 2.9 equiv.) and hydrazine monohydrate $(1.57 \mathrm{~mL}, 1.61 \mathrm{~g}, 32.2$ mmol, 2.4 equiv.). The mixture was heated at reflux overnight, then cooled in an ice bath for $15 \mathrm{~min}$, filtered through a sintered funnel and washed with cold $\mathrm{H}_{2} \mathrm{O}(25 \mathrm{~mL} \times 3)$, then dried in vacuo to give $\mathbf{S 5}$ as a pale yellow solid (1.62 g, $11.2 \mathrm{mmol}, 83 \%) . \operatorname{mp~} 168-170{ }^{\circ} \mathrm{C}$. ${ }^{1} \mathrm{H}$ NMR $\left(500 \mathrm{MHz}, \mathrm{DMSO}-d_{6}\right): \delta 8.14(\mathrm{~s}$, $1 \mathrm{H}), 8.02(\mathrm{~d}, J=1.4 \mathrm{~Hz}, 1 \mathrm{H}), 7.93(\mathrm{~d}, J$ $=1.4 \mathrm{~Hz}, 1 \mathrm{H}), 4.32(\mathrm{~s}, 2 \mathrm{H}) \cdot{ }^{13} \mathrm{C}\left\{{ }^{1} \mathrm{H}\right\} \mathrm{NMR}$ $\left(126 \mathrm{MHz}, \mathrm{DMSO}-d_{6}\right)$ : $\delta$ 156.6, 140.3, 133.9,
129.5. HRMS (ESI/FTICR) $m / z:[\mathrm{M}+\mathrm{H}]^{+}$ calcd for $\mathrm{C}_{4} \mathrm{H}_{6} \mathrm{ClN}_{4}$ 145.02755; found 145.02749. The spectroscopic data were in agreement with those in the literature. ${ }^{32}$

\section{5-Chloro-3-(4-(difluoromethoxy)phenyl)-}

[1,2,4]triazolo/4,3-a]pyrazine (16). General Procedure $\mathrm{C}$ was applied using S1 (1.51 g, 10.4 mmol, 1.0 equiv.) and 4(difluoromethoxy)benzaldehyde (1.98 g, 11.5 mmol, 1.1 equiv.) to give $\mathbf{1 6}$ as a brown solid (2.26 g, $7.62 \mathrm{mmol}, 73 \%) . \quad \mathrm{mp} 124-126{ }^{\circ} \mathrm{C}$. ${ }^{1} \mathrm{H}$ NMR $\left(500 \mathrm{MHz}, \mathrm{DMSO}-d_{6}\right): \delta 9.47(\mathrm{~s}, 1 \mathrm{H})$, $8.08(\mathrm{~s}, 1 \mathrm{H}), 7.79(\mathrm{~d}, J=8.2 \mathrm{~Hz}, 2 \mathrm{H}), 7.41$ $(\mathrm{t}, J=73.6 \mathrm{~Hz}, 1 \mathrm{H}), 7.36(\mathrm{~d}, J=8.2 \mathrm{~Hz}$, 2H). ${ }^{13} \mathrm{C}\left\{{ }^{1} \mathrm{H}\right\}$ NMR $\left(126 \mathrm{MHz}\right.$, DMSO- $\left.d_{6}\right): \delta$ $153.3-152.1(\mathrm{~m}), 147.0,146.7,142.7,133.3$, $129.2,124.0,121.8,117.4,116.2$ (t, $J=258.0$ $\mathrm{Hz})\left(\mathrm{OCHF}_{2}\right)$. HRMS (ESI/FTICR) $m / z$ : $[\mathrm{M}+\mathrm{H}]^{+}$calcd for $\mathrm{C}_{12} \mathrm{H}_{8} \mathrm{ClF}_{2} \mathrm{~N}_{4} \mathrm{O}$ 297.03492; found 297.03460.

3-(4-(Difluoromethoxy)phenyl)-5-(phenethylthio)-[1,2,4]triazolo[4,3-a]pyrazine (17). General Procedure D was applied using 16 (101 mg, $0.341 \mathrm{mmol}, 1.0$ equiv.) and 2-phenylethane-1thiol (47.1 mg, $0.341 \mathrm{mmol}, 1.0$ equiv.). Fractions corresponding to the second peak were evaporated to give $\mathbf{1 7}$ as a yellow solid (11.0 $\mathrm{mg}, 0.0276 \mathrm{mmol}, 8 \%$ ). $\mathrm{mp} 78-83{ }^{\circ} \mathrm{C} .{ }^{1} \mathrm{H} \mathrm{NMR}$ $\left(400 \mathrm{MHz} \mathrm{CDCl}_{3}\right): \delta 9.21(\mathrm{~s}, 1 \mathrm{H}), 7.76(\mathrm{~s}, 1 \mathrm{H})$, $7.68-7.60(\mathrm{~m}, 2 \mathrm{H}), 7.28-7.15(\mathrm{~m}, 5 \mathrm{H}), 7.02$ $-6.94(\mathrm{~m}, 2 \mathrm{H}), 6.64(\mathrm{t}, J=73.1 \mathrm{~Hz}, 1 \mathrm{H}), 2.92$ (t, $J=7.5 \mathrm{~Hz}, 2 \mathrm{H}), 2.76(\mathrm{t}, J=7.4 \mathrm{~Hz}, 2 \mathrm{H})$. ${ }^{13} \mathrm{C}\left\{{ }^{1} \mathrm{H}\right\}$ NMR $\left(101 \mathrm{MHz}, \mathrm{CDCl}_{3}\right): \delta 153.10$ $(\mathrm{t}, J=2.8 \mathrm{~Hz}), 147.6,146.4,142.3,138.3$, $133.5,131.3,128.8,128.6,128.4,127.1,124.1$, 118.3, 115.65 (t, $J=261.3 \mathrm{~Hz}), 35.8,34.6$. HRMS (ESI/FTICR) $m / z:[\mathrm{M}+\mathrm{H}]^{+}$calcd for $\mathrm{C}_{20} \mathrm{H}_{17} \mathrm{~F}_{2} \mathrm{~N}_{4} \mathrm{OS}$ 399.10856; found 399.10801.

3-(4-(Difluoromethoxy)phenyl)-8-(phenethylthio)-[1,2,4]triazolo[4,3-a]-pyrazine (18). Isolated from the same reaction as for 17. Fractions corresponding to the first peak were evaporated to give to give $\mathbf{1 8}$ as an off-white solid (113 mg, $0.284 \mathrm{mmol}, 83 \%$ ). $\operatorname{mp~} 156-158{ }^{\circ} \mathrm{C}$. ${ }^{1} \mathrm{H}$ NMR $\left(500 \mathrm{MHz}\right.$, DMSO- $\left.d_{6}\right): \delta 8.32(\mathrm{~d}, J$ $=4.8 \mathrm{~Hz}, 1 \mathrm{H}), 8.04-7.96(\mathrm{~m}, 2 \mathrm{H}), 7.83(\mathrm{~d}$, $J=4.8 \mathrm{~Hz}, 1 \mathrm{H}), 7.58-7.39(\mathrm{~m}, 5 \mathrm{H}), 7.37-$ $7.29(\mathrm{~m}, 4 \mathrm{H}), 7.30-7.20(\mathrm{~m}, 1 \mathrm{H}), 3.59(\mathrm{dd}$, $J=8.4,6.7 \mathrm{~Hz}, 2 \mathrm{H}), 3.05(\mathrm{dd}, J=8.4,6.7$ 
$\mathrm{Hz}, 2 \mathrm{H}) \cdot{ }^{13} \mathrm{C}\left\{{ }^{1} \mathrm{H}\right\}$ NMR $\left(126 \mathrm{MHz}\right.$, DMSO- $\left.d_{6}\right)$ : $\delta$ 153.0, $152.4(\mathrm{t}, J=3.3 \mathrm{~Hz}), 146.9,143.8$, $139.9,130.2,129.5,128.6,128.4,126.4,122.5$, $119.2,116.1$ (t, $J=258.5 \mathrm{~Hz}), 113.2,34.4$, 29.4. ${ }^{19} \mathrm{~F}$ NMR (471 MHz, DMSO- $\left.d_{6}\right): \delta-82.8$. HRMS (ESI/FTICR) $m / z$ : $[\mathrm{M}+\mathrm{H}]^{+}$calcd for $\mathrm{C}_{20} \mathrm{H}_{17} \mathrm{~F}_{2} \mathrm{~N}_{4} \mathrm{OS}$ 399.10856; found 399.10827.

8-Chloro-[1,2,4]triazolo[4,3-a]pyrazine (25). General Procedure B was applied using $\mathbf{S 4}$ ( $2.71 \mathrm{~g}, 18.8 \mathrm{mmol})$ to give $\mathbf{2 5}$ as a yellow solid (0.870 g, $5.63 \mathrm{mmol}, 30 \%$ ). $\operatorname{mp} 203-206{ }^{\circ} \mathrm{C}$ (lit. $\left.{ }^{30} \mathrm{mp} 205{ }^{\circ} \mathrm{C}\right) .{ }^{1} \mathrm{H} \mathrm{NMR}\left(200 \mathrm{MHz}, \mathrm{CDCl}_{3}\right)$ : $\delta 9.00(\mathrm{~s}, 1 \mathrm{H}), 8.05(\mathrm{~d}, J=4.7 \mathrm{~Hz}, 1 \mathrm{H}), 7.74(\mathrm{~d}$, $J=4.7 \mathrm{~Hz}, 1 \mathrm{H}$ ). HRMS (ESI/FTICR) $\mathrm{m} / z$ : $[\mathrm{M}+\mathrm{Na}]^{+}$calcd for $\mathrm{C}_{5} \mathrm{H}_{3} \mathrm{ClN}_{4} \mathrm{Na} 176.99384$; found 176.99367. The spectroscopic data and melting point were in agreement with those in the literature. ${ }^{30,33}$

\section{8-Chloro-3-phenyl-[1,2,4]triazolo[4,3-a]pyra-} zine (26). General Procedure $\mathrm{C}$ was applied using S4 (0.768 g, $5.31 \mathrm{mmol}, 1.0$ equiv.) and benzaldehyde $(0.620 \mathrm{~g}, 5.84 \mathrm{mmol}, 1.1$ equiv.) to give $\mathbf{2 6}$ as a white solid (0.976 g, $4.23 \mathrm{mmol}$, $80 \%$ ). $\operatorname{mp~} 192-195{ }^{\circ} \mathrm{C}$ (lit. ${ }^{34} \mathrm{mp} 193-195{ }^{\circ} \mathrm{C}$ ). ${ }^{1} \mathrm{H} \mathrm{NMR}\left(200 \mathrm{MHz}, \mathrm{CDCl}_{3}\right): \delta 8.16(\mathrm{~d}, J$ $=4.8 \mathrm{~Hz}, 1 \mathrm{H}), 7.92-7.78(\mathrm{~m}, 2 \mathrm{H}), 7.72(\mathrm{~d}$, $J=4.8 \mathrm{~Hz}, 1 \mathrm{H}), 7.64$ (q, $J=3.1 \mathrm{~Hz}, 3 \mathrm{H})$. HRMS (ESI/FTICR) $m / z:[\mathrm{M}+\mathrm{Na}]^{+}$calcd for $\mathrm{C}_{11} \mathrm{H}_{7} \mathrm{ClN}_{4} \mathrm{Na} 253.02514$; found 253.02522 . The spectroscopic data and melting point were in agreement with those in the literature. ${ }^{34}$

8-Chloro-3-(4-nitrophenyl)-[1,2,4]triazolo-

[4,3-a]pyrazine (27). General Procedure C was applied using $\mathbf{S} 4(0.655 \mathrm{~g}, 4.53 \mathrm{mmol}$, 1.0 equiv.) and 4-nitrobenzaldehyde (0.754 g, 4.99 mmol, 1.1 equiv.) to give $\mathbf{2 7}$ as a yellow solid (1.15 g, $4.16 \mathrm{mmol}, 92 \%)$. m.p. 231-234 ${ }^{\circ} \mathrm{C}$ (decomp.) (lit. ${ }^{22} \mathrm{mp} 201-204{ }^{\circ} \mathrm{C}$ ). ${ }^{1} \mathrm{H}$ NMR $\left(500 \mathrm{MHz}\right.$, DMSO- $\left.d_{6}\right): \delta 8.77(\mathrm{~d}$, $J=4.8 \mathrm{~Hz}, 1 \mathrm{H}), 8.47(\mathrm{~d}, J=8.6 \mathrm{~Hz}, 2 \mathrm{H})$, $8.26(\mathrm{~d}, J=8.6 \mathrm{~Hz}, 2 \mathrm{H}), 7.89(\mathrm{~d}, J=4.8$ $\mathrm{Hz}, 1 \mathrm{H}) .{ }^{13} \mathrm{C}\left\{{ }^{1} \mathrm{H}\right\}$ NMR $(126 \mathrm{MHz}$, DMSO$\left.d_{6}\right): \delta 148.9,147.6,144.6,142.5,132.0,130.1$, 129.6, 124.8, 118.4. HRMS (ESI/FTICR) $m / z$ : $[\mathrm{M}+\mathrm{H}]^{+}$calcd for $\mathrm{C}_{11} \mathrm{H}_{7} \mathrm{ClN}_{5} \mathrm{O}_{2}$ 298.01022; found 298.01025. The spectroscopic data were in agreement with the literature, but the melting point was significantly higher. ${ }^{22}$

8-Phenethoxy-[1,2,4]triazolo[4,3-a]pyrazine
(28). General Procedure D was applied using 25 (104 mg, $0.673 \mathrm{mmol}, 1.0$ equiv.) and 2-phenylethan-1-ol ( $82.2 \mathrm{mg}, 0.673 \mathrm{mmol}, 1.0$ equiv.) to give $\mathbf{2 8}$ as an off-white solid (83.0 $\mathrm{mg}, \quad 0.345 \mathrm{mmol}, 51 \%)$. $\mathrm{mp} 161-162{ }^{\circ} \mathrm{C}$. ${ }^{1} \mathrm{H}$ NMR (500 MHz, DMSO- $\left.d_{6}\right): \delta 9.36$ (s, $1 \mathrm{H}), 8.19(\mathrm{~d}, J=4.7 \mathrm{~Hz}, 1 \mathrm{H}), 7.42(\mathrm{~d}, J=$ $4.7 \mathrm{~Hz}, 1 \mathrm{H}), 7.38-7.27(\mathrm{~m}, 4 \mathrm{H}), 7.26-7.19$ $(\mathrm{m}, 1 \mathrm{H}), 4.72(\mathrm{t}, J=6.9 \mathrm{~Hz}, 2 \mathrm{H}), 3.16(\mathrm{t}$, $J=6.9 \mathrm{~Hz}, 2 \mathrm{H}) .{ }^{13} \mathrm{C}\left\{{ }^{1} \mathrm{H}\right\} \mathrm{NMR}(126 \mathrm{MHz}$, DMSO- $\left.d_{6}\right): \delta 152.6,138.7,138.4,137.9,128.9$, $128.4,126.6,126.4,113.2,113.2,67.2,34.2$. HRMS (ESI/FTICR) $m / z:[\mathrm{M}+\mathrm{Na}]^{+}$calcd for $\mathrm{C}_{13} \mathrm{H}_{12} \mathrm{~N}_{4} \mathrm{ONa}$ 263.09033; found 263.08997.

8-(Phenethylthio)-[1,2,4]triazolo[4,3-a]pyrazine (29). General Procedure D was applied using 25 (104 mg, $0.673 \mathrm{mmol}, 1.0$ equiv.) and 2-phenylethane-1-thiol (93.0 mg, $0.673 \mathrm{mmol}$, 1.0 equiv.) to give $\mathbf{2 9}$ as an off-white solid (154 mg, $0.602 \mathrm{mmol}, 90 \%$ ). $\operatorname{mp~} 148-150{ }^{\circ} \mathrm{C}$. ${ }^{1} \mathrm{H}$ NMR (500 MHz, DMSO- $\left.d_{6}\right): \delta 9.38(\mathrm{~d}, J=$ $0.8 \mathrm{~Hz}, 1 \mathrm{H}), 8.33(\mathrm{dd}, J=4.6,0.8 \mathrm{~Hz}, 1 \mathrm{H}), 7.79$ $(\mathrm{dd}, J=4.7,0.8 \mathrm{~Hz}, 1 \mathrm{H}), 7.31(\mathrm{~d}, J=4.4 \mathrm{~Hz}$, $4 \mathrm{H}), 7.23(\mathrm{~h}, J=4.0 \mathrm{~Hz}, 1 \mathrm{H}), 3.59-3.53(\mathrm{~m}$, 2H), $3.06-3.00(\mathrm{~m}, 2 \mathrm{H}) .{ }^{13} \mathrm{C}\left\{{ }^{1} \mathrm{H}\right\}$ NMR (126 MHz, DMSO-d $\left.d_{6}\right): \delta 152.2,142.7,139.9,138.1$, 128.7, 128.6, 128.4, 126.4, 114.3, 34.4, 29.4. HRMS (ESI/FTICR) $m / z:[\mathrm{M}+\mathrm{Na}]^{+}$calcd for $\mathrm{C}_{13} \mathrm{H}_{12} \mathrm{~N}_{4} \mathrm{SNa} 279.06749$; found 279.06714 .

N-Phenethyl-[1,2,4]triazolo[4,3-a]pyrazin-8amine (30). Preparation 1: General Procedure E was applied using 25 (104 mg, 0.654 mmol) and 2-phenylethan-1-amine (244 mg, 2.01 mmol, 3.0 equiv.) to give $\mathbf{3 0}$ as an offwhite solid (135 mg, $0.564 \mathrm{mmol}, 84 \%$ ). Preparation 2: General Procedure E was applied using 45a (100 mg, $0.649 \mathrm{mmol}, 1.0$ equiv.) and 2-phenylethan-1-amine (235 mg, $1.95 \mathrm{mmol}, 3.0$ equiv.) to give $\mathbf{3 0}$ as an off-white solid (102 mg, $0.424 \mathrm{mmol}, 65 \%)$. mp $191-193{ }^{\circ} \mathrm{C}$ (decomp.). ${ }^{1} \mathrm{H}$ NMR $\left(500 \mathrm{MHz}, \mathrm{DMSO}-d_{6}\right): \delta 9.19(\mathrm{~s}, 1 \mathrm{H})$, $8.16(\mathrm{t}, J=5.8 \mathrm{~Hz}, 1 \mathrm{H}), 7.74(\mathrm{~d}, J=4.7 \mathrm{~Hz}$, $1 \mathrm{H}), 7.32-7.23(\mathrm{~m}, 5 \mathrm{H}), 7.23-7.15(\mathrm{~m}, 1 \mathrm{H})$, $3.71(\mathrm{q}, J=6.9 \mathrm{~Hz}, 2 \mathrm{H}), 2.99-2.92(\mathrm{~m}, 2 \mathrm{H})$. ${ }^{13} \mathrm{C}\left\{{ }^{1} \mathrm{H}\right\}$ NMR $\left(126 \mathrm{MHz}, \mathrm{DMSO}-d_{6}\right): \delta 147.4$, $139.5,138.6,138.1,129.1,128.6,128.3,126.0$, 107.2, 41.6, 34.5. HRMS (ESI/FTICR) $\mathrm{m} / z$ : $[\mathrm{M}+\mathrm{H}]^{+}$calcd for $\mathrm{C}_{13} \mathrm{H}_{14} \mathrm{~N}_{5}$ 240.12437; found 240.12406 . 
8-Phenethoxy-3-phenyl-[1,2,4]triazolo[4,3-

alpyrazine (31). General Procedure D was applied using 26 (115 mg, 0.499 mmol, 1.0 equiv.) and 2-phenylethan-1-ol (60.9 mg, 0.499 mmol, 1.0 equiv.) to give $\mathbf{3 1}$ as a white solid (91.0 mg, $0.288 \mathrm{mmol}, 58 \%$ ). $\operatorname{mp~} 145-147{ }^{\circ} \mathrm{C}$. ${ }^{1} \mathrm{H}$ NMR $\left(500 \mathrm{MHz}\right.$, DMSO- $\left.d_{6}\right): \delta 8.19(\mathrm{~d}, J$ $=4.9 \mathrm{~Hz}, 1 \mathrm{H}), 7.94-7.88(\mathrm{~m}, 2 \mathrm{H}), 7.68-$ $7.59(\mathrm{~m}, 3 \mathrm{H}), 7.47(\mathrm{~d}, J=4.9 \mathrm{~Hz}, 1 \mathrm{H}), 7.41$ $-7.29(\mathrm{~m}, 4 \mathrm{H}), 7.24(\mathrm{t}, J=7.3 \mathrm{~Hz}, 1 \mathrm{H}), 4.76$ (t, $J=6.8 \mathrm{~Hz}, 2 \mathrm{H}), 3.19(\mathrm{t}, J=6.9 \mathrm{~Hz}, 2 \mathrm{H})$. ${ }^{13} \mathrm{C}\left\{{ }^{1} \mathrm{H}\right\} \mathrm{NMR}\left(126 \mathrm{MHz}, \mathrm{DMSO}-d_{6}\right): \delta 153.2$, 148.0, 139.7, 138.0, 130.5, 129.3, 129.0, 128.4, 128.1, 127.4, 126.4, 125.9, 112.1, 67.4, 34.2. HRMS (ESI/FTICR) $m / z:[\mathrm{M}+\mathrm{Na}]^{+}$calcd for $\mathrm{C}_{19} \mathrm{H}_{16} \mathrm{~N}_{4} \mathrm{ONa} 339.12163$; found 339.12165.

8-(Phenethylthio)-3-phenyl-[1,2,4]triazolo[4,3-a]pyrazine (32). General Procedure D was applied using $\mathbf{2 6}$ (107 $\mathrm{mg}, 0.464 \mathrm{mmol}$ ) and 2-phenylethane-1-thiol (65.1 mg, $0.464 \mathrm{mmol}$, 1.0 equiv.) to give 32 as an off-white solid (145 mg, $0.440 \mathrm{mmol}, 94 \%$ ). $\operatorname{mp~} 154-156{ }^{\circ} \mathrm{C}$. ${ }^{1} \mathrm{H}$ NMR $\left(500 \mathrm{MHz}\right.$, DMSO- $\left.d_{6}\right): \delta 8.33(\mathrm{~d}, J$ $=4.8 \mathrm{~Hz}, 1 \mathrm{H}), 7.97-7.88(\mathrm{~m}, 2 \mathrm{H}), 7.83(\mathrm{~d}, J$ $=4.8 \mathrm{~Hz}, 1 \mathrm{H}), 7.69-7.59(\mathrm{~m}, 3 \mathrm{H}), 7.33(\mathrm{~d}, J$ $=5.0 \mathrm{~Hz}, 4 \mathrm{H}), 7.24(\mathrm{ddd}, J=8.8,5.3,3.5 \mathrm{~Hz}$, $1 \mathrm{H}), 3.63-3.56(\mathrm{~m}, 2 \mathrm{H}), 3.09-3.02(\mathrm{~m}, 2 \mathrm{H})$. ${ }^{13} \mathrm{C}\left\{{ }^{1} \mathrm{H}\right\} \mathrm{NMR}\left(126 \mathrm{MHz}, \mathrm{DMSO}-d_{6}\right): \delta 153.0$, 147.6, 143.8, 140.0, 130.5, 129.5, 129.3, 128.6, 128.4, 128.2, 126.4, 125.7, 113.2, 34.4, 29.4. HRMS (ESI/FTICR) $m / z:[\mathrm{M}+\mathrm{H}]^{+}$calcd for $\mathrm{C}_{19} \mathrm{H}_{17} \mathrm{~N}_{4} \mathrm{~S}$ 333.11684; found 333.11641.

N-Phenethyl-3-phenyl-[1,2,4]triazolo/4,3-

alpyrazin-8-amine (33). General Procedure E was applied using 26 (102 $\mathrm{mg}, 0.442 \mathrm{mmol}$ ) and 2-phenylethan-1-amine (161 mg, $1.33 \mathrm{mmol}, 3.0$ equiv.) to give 33 (120 mg, $0.381 \mathrm{mmol}, 86 \%)$. mp 206-209 ${ }^{\circ} \mathrm{C} .{ }^{1} \mathrm{H}$ NMR $(500 \mathrm{MHz}$, DMSO$\left.d_{6}\right): \delta 8.27(\mathrm{t}, J=5.7 \mathrm{~Hz}, 1 \mathrm{H}), 7.92-7.86(\mathrm{~m}$, $2 \mathrm{H}), 7.75(\mathrm{~d}, J=4.8 \mathrm{~Hz}, 1 \mathrm{H}), 7.67-7.57(\mathrm{~m}$, $3 \mathrm{H}), 7.36(\mathrm{~d}, J=4.8 \mathrm{~Hz}, 1 \mathrm{H}), 7.34-7.25(\mathrm{~m}$, $4 \mathrm{H}), 7.25-7.17(\mathrm{~m}, 1 \mathrm{H}), 3.79-3.71(\mathrm{~m}, 2 \mathrm{H})$, $3.02-2.95(\mathrm{~m}, 2 \mathrm{H}) \cdot{ }^{13} \mathrm{C}\left\{{ }^{1} \mathrm{H}\right\} \mathrm{NMR}(126 \mathrm{MHz}$, DMSO- $\left.d_{6}\right): \delta 147.9,147.7,139.6,139.5,130.2$, 130.2, 129.3, 128.7, 128.3, 128.0, 126.3, 126.1, 106.0, 41.6, 34.5. HRMS (ESI/FTICR) $m / z$ : $[\mathrm{M}+\mathrm{H}]^{+}$calcd for $\mathrm{C}_{19} \mathrm{H}_{18} \mathrm{~N}_{5} 316.15567$; found 316.15526 .

3-(4-Nitrophenyl)-8-phenethoxy-[1,2,4]tria- zolo[4,3-a]pyrazine (34). Preparation 1: General Procedure D was applied using 27 (113 mg, $0.410 \mathrm{mmol}, 1.0$ equiv.) and 2-phenylethan-1-ol (50.1 mg, $0.410 \mathrm{mmol}, 1.0$ equiv.) to give $\mathbf{3 4}$ as a yellow solid (125 mg, $0.346 \mathrm{mmol}, 84 \%$ ). Preparation 2: isolated from the same reaction as for 46e preparation 1: fractions correspond to the first peak were evaporated to give $\mathbf{3 4}$ as a yellow solid (2.05 mg, $5.51 \mu \mathrm{mol}, 2 \%)$. mp 238 $240{ }^{\circ} \mathrm{C}$ (decomp.). ${ }^{1} \mathrm{H}$ NMR $(500 \mathrm{MHz}$, DMSO$\left.d_{6}\right): \delta 8.45(\mathrm{~d}, J=8.3 \mathrm{~Hz}, 2 \mathrm{H}), 8.32(\mathrm{~d}, J=4.9$ $\mathrm{Hz}, 1 \mathrm{H}), 8.24(\mathrm{~d}, J=8.4 \mathrm{~Hz}, 2 \mathrm{H}), 7.56(\mathrm{~d}, J=$ $4.9 \mathrm{~Hz}, 1 \mathrm{H}), 7.38(\mathrm{~d}, J=7.6 \mathrm{~Hz}, 2 \mathrm{H}), 7.33$ (t, $J=7.5 \mathrm{~Hz}, 2 \mathrm{H}), 7.24(\mathrm{t}, J=7.2 \mathrm{~Hz}, 1 \mathrm{H}), 4.79$ $(\mathrm{t}, J=6.8 \mathrm{~Hz}, 2 \mathrm{H}), 3.20(\mathrm{t}, J=6.8 \mathrm{~Hz}, 2 \mathrm{H})$. ${ }^{13} \mathrm{C}\left\{{ }^{1} \mathrm{H}\right\} \mathrm{NMR}\left(126 \mathrm{MHz}, \mathrm{DMSO}-d_{6}\right): \delta 153.2$, $148.2,146.5,140.1,137.9,132.0,129.4,128.9$, $128.4,127.9,126.4,124.3,112.4,67.5,34.2$. HRMS (ESI/FTICR) $m / z:[\mathrm{M}+\mathrm{H}]^{+}$calcd for $\mathrm{C}_{19} \mathrm{H}_{16} \mathrm{~N}_{5} \mathrm{O}_{3} 362.12477$; found 362.12462 .

3-(4-Nitrophenyl)-8-(phenethylthio)-[1,2,4]triazolo[4,3-a]pyrazine (35). Preparation 1: General Procedure D was applied using $\mathbf{2 7}$ (107 mg, $0.390 \mathrm{mmol}$ ) and 2-phenylethane-1thiol (65.7 mg, $0.390 \mathrm{mmol}, 1.0$ equiv.) to give 35 as a yellow solid (103 mg, $0.273 \mathrm{mmol}$, $70 \%$ ). Preparation 2: Isolated from the same reaction as for $\mathbf{4 6 \mathbf { j }}$. Fractions corresponding to the first peak were evaporated to give $\mathbf{3 5}$ as a yellow solid (66.2 mg, $0.175 \mathrm{mmol}, 44 \%$ ). mp 236-238 ${ }^{\circ} \mathrm{C}$ (decomp.). ${ }^{1} \mathrm{H}$ NMR (500 MHz, DMSO- $\left.d_{6}\right): \delta 8.46(\mathrm{dd}, J=6.9,2.0 \mathrm{~Hz}, 3 \mathrm{H})$, $8.28-8.22(\mathrm{~m}, 2 \mathrm{H}), 7.92(\mathrm{~d}, J=4.8 \mathrm{~Hz}$, $1 \mathrm{H}), 7.37-7.30(\mathrm{~m}, 4 \mathrm{H}), 7.29-7.21(\mathrm{~m}, 1 \mathrm{H})$, $3.61(\mathrm{dd}, J=8.4,6.7 \mathrm{~Hz}, 2 \mathrm{H}), 3.07$ (dd, $J$ $=8.4,6.7 \mathrm{~Hz}, 2 \mathrm{H}) .{ }^{13} \mathrm{C}\left\{{ }^{1} \mathrm{H}\right\} \mathrm{NMR}(126 \mathrm{MHz}$, DMSO- $\left.d_{6}\right): \delta 153.1,148.3,146.2,144.2,139.9$, 131.8, 130.0, 129.5, 128.6, 128.4, 126.4, 124.3, 113.5, 34.4, 29.5. HRMS (ESI/FTICR) $m / z$ : $[\mathrm{M}+\mathrm{H}]^{+}$calcd for $\mathrm{C}_{19} \mathrm{H}_{16} \mathrm{~N}_{5} \mathrm{O}_{2} \mathrm{~S}$ 378.10192; found 378.10180 .

3-(4-Nitrophenyl)-N-phenethyl-[1,2,4]triazolo[4,3-a]pyrazin-8-amine (36). Preparation 1: General Procedure E was applied using 27 (112 mg, $0.406 \mathrm{mmol}, 1.0$ equiv.) and 2-phenylethan-1-amine (148 mg, $1.22 \mathrm{mmol}$, 3.0 equiv.) to give 36 (127 mg, $0.352 \mathrm{mmol}$, 87\%). Preparation 2: General Procedure E was applied using 45c (103 mg, $0.374 \mathrm{mmol}$, 
1.0 equiv.) and 2-phenylethan-1-amine (136 $\mathrm{mg}, 1.12 \mathrm{mmol}, 3.0$ equiv.) to give 36 as a yellow solid (133 mg, $0.369 \mathrm{mmol}, 99 \%$ ). mp 236-238 ${ }^{\circ} \mathrm{C}$ (decomp.). ${ }^{1} \mathrm{H}$ NMR (500 MHz, DMSO- $\left.d_{6}\right): \delta 8.47-8.41(\mathrm{~m}, 2 \mathrm{H}), 8.38(\mathrm{t}, J=$ $5.8 \mathrm{~Hz}, 1 \mathrm{H}), 8.29-8.20(\mathrm{~m}, 2 \mathrm{H}), 7.88(\mathrm{~d}, J=$ $4.8 \mathrm{~Hz}, 1 \mathrm{H}), 7.45$ (d, $J=4.8 \mathrm{~Hz}, 1 \mathrm{H}), 7.30$ (h, $J=5.9 \mathrm{~Hz}, 4 \mathrm{H}), 7.21$ (tt, $J=5.9,2.1 \mathrm{~Hz}, 1 \mathrm{H})$, $3.76(\mathrm{q}, J=6.8 \mathrm{~Hz}, 2 \mathrm{H}), 3.02-2.96(\mathrm{~m}, 2 \mathrm{H})$. ${ }^{13} \mathrm{C}\left\{{ }^{1} \mathrm{H}\right\}$ NMR $\left(126 \mathrm{MHz}, \mathrm{DMSO}-d_{6}\right): \delta$ 148.0, $147.9,146.1,139.9,139.5,132.4,130.8,129.1$, 128.7, 128.3, 126.1, 124.3, 106.2, 41.6, 34.4. HRMS (ESI/FTICR) $m / z:[\mathrm{M}+\mathrm{H}]^{+}$calcd for $\mathrm{C}_{19} \mathrm{H}_{17} \mathrm{~N}_{6} \mathrm{O}_{2}$ 361.14075; found 361.14041.

6-Chloro-[1,2,4]triazolo[4,3-a]pyrazine (37). General Procedure B was applied using S5 (1.53 g, $10.6 \mathrm{mmol})$ to give 37 as an orange solid (0.800 g, $5.18 \mathrm{mmol}, 49 \%)$. $\mathrm{mp}$ 215-217 ${ }^{\circ} \mathrm{C}$ (decomp.). ${ }^{1} \mathrm{H}$ NMR $(500 \mathrm{MHz}$, DMSO- $\left.d_{6}\right): \quad \delta 9.41(\mathrm{~d}, J=0.7 \mathrm{~Hz}, 1 \mathrm{H})$, $9.36(\mathrm{dd}, J=1.5,0.7 \mathrm{~Hz}, 1 \mathrm{H}), 8.90(\mathrm{~d}, J$ $=1.5 \mathrm{~Hz}, 1 \mathrm{H}) .{ }^{13} \mathrm{C}\left\{{ }^{1} \mathrm{H}\right\} \mathrm{NMR}(126 \mathrm{MHz}$, DMSO- $\left.d_{6}\right): \delta 143.9,143.0,137.3,133.4,116.3$. HRMS (ESI/FTICR) $m / z:[\mathrm{M}+\mathrm{H}]^{+}$calcd for $\mathrm{C}_{5} \mathrm{H}_{4} \mathrm{ClN}_{4}$ 155.01190; found 155.01178.

6-Chloro-3-(4-(difluoromethoxy)phenyl)-

[1,2,4]triazolo[4,3-a]pyrazine (38). General Procedure $\mathrm{C}$ was applied using S5 (1.33 g, $9.23 \mathrm{mmol}, 1.0$ equiv.) and 4(difluoromethoxy)benzaldehyde (1.22 mL, 1.59 g, 9.23 mmol, 1.1 equiv.) to give $\mathbf{2 7}$ as a pale brown solid (1.75 g, $5.89 \mathrm{mmol}, 64 \%)$. mp 159$161{ }^{\circ} \mathrm{C} .{ }^{1} \mathrm{H}$ NMR $\left(500 \mathrm{MHz}, \mathrm{DMSO}-d_{6}\right): \delta 9.41$ $(\mathrm{s}, 1 \mathrm{H}), 8.85(\mathrm{~s}, 1 \mathrm{H}), 8.05(\mathrm{~d}, J=8.6 \mathrm{~Hz}, 2 \mathrm{H})$, $7.43(\mathrm{~d}, J=8.4 \mathrm{~Hz}, 2 \mathrm{H}), 7.41$ (t, $J=73.5$ $\mathrm{Hz}, 1 \mathrm{H}) .{ }^{13} \mathrm{C}\left\{{ }^{1} \mathrm{H}\right\}$ NMR $\left(126 \mathrm{MHz}, \mathrm{DMSO}-d_{6}\right)$ : $\delta 152.5(\mathrm{t}, J=3.3 \mathrm{~Hz}), 146.2,145.2,143.4$, $134.6,130.3,122.1,119.2,116.1$ (t, $J=258.6$ $\mathrm{Hz}), 115.2 .{ }^{19} \mathrm{~F}$ NMR $\left(471 \mathrm{MHz}, \mathrm{DMSO}-d_{6}\right): \delta$ -82.8. HRMS (ESI/FTICR) $m / z:[\mathrm{M}+\mathrm{Na}]^{+}$ calcd for $\mathrm{C}_{12} \mathrm{H}_{7} \mathrm{ClF}_{2} \mathrm{~N}_{4} \mathrm{ONa} 319.01687$; found 319.01690.

2,6-Diiodopyrazine (41). Compounds was prepared following literature procedures. ${ }^{35} \mathrm{Hy}-$ droiodic acid ( $50 \%$ solution, $25 \mathrm{~mL}, 5.0$ equiv.) was added to 2,6-dichloropyrazine (5.07 g, 34.0 mmol, 1.0 equiv.) and $\mathrm{NaI}$ (6.63 g, $44.2 \mathrm{mmol}$, 1.3 equiv.) in a sealed tube and heated at $100{ }^{\circ} \mathrm{C}$ for $3 \mathrm{~h}$. The reaction was cooled to room temperature and diluted with $\mathrm{Et}_{2} \mathrm{O}(200 \mathrm{~mL})$. The solution was washed with $\mathrm{H}_{2} \mathrm{O}(100 \mathrm{~mL} \times$ 2), sat. aq. $\mathrm{NaHCO}_{3}(50 \mathrm{~mL})$, sat. aq. $\mathrm{Na}_{2} \mathrm{~S}_{2} \mathrm{O}_{3}$ $(50 \mathrm{~mL})$, brine $(30 \mathrm{~mL})$, dried $\left(\mathrm{Na}_{2} \mathrm{SO}_{4}\right)$, filtered and concentrated under reduced pressure to give $\mathbf{4 1}$ as a white solid (9.91 g, $29.9 \mathrm{mmol}$, $88 \%) . \quad m p \quad 90-92{ }^{\circ} \mathrm{C} .{ }^{1} \mathrm{H}$ NMR $(300 \mathrm{MHz}$, $\left.\mathrm{CDCl}_{3}\right): \quad \delta 8.74(\mathrm{~s}, 2 \mathrm{H}) \cdot{ }^{13} \mathrm{C}\left\{{ }^{1} \mathrm{H}\right\} \operatorname{NMR}(75$ $\left.\mathrm{MHz}, \mathrm{CDCl}_{3}\right): \delta 151.2,116.8$. The spectroscopic data were in agreement with those in the literature. $^{35}$

2-Chloro-6-phenethoxypyrazine (42). General Procedure D was applied using 2,6dichloropyrazine (107 mg, $0.718 \mathrm{mmol}, 1.0$ equiv.) and 2-phenylethan-1-ol (87.8 mg, $0.718 \mathrm{mmol}, 1.0$ equiv.). The reaction mixture was purified by FCC on silica using a gradient of EtOAc $(0 \%$ to $6 \%)$ in hexanes to give 42 as a colourless oil (137 mg, $0.582 \mathrm{mmol}$, 81\%). ${ }^{1} \mathrm{H} \mathrm{NMR}\left(500 \mathrm{MHz}, \mathrm{CDCl}_{3}\right): \delta 8.13$ $(\mathrm{s}, 1 \mathrm{H}), 8.11(\mathrm{~s}, 1 \mathrm{H}), 7.36-7.20(\mathrm{~m}, 5 \mathrm{H})$, $4.56(\mathrm{t}, J=7.0 \mathrm{~Hz}, 2 \mathrm{H}), 3.11(\mathrm{t}, J=7.0$ $\mathrm{Hz}, 2 \mathrm{H}) \cdot{ }^{13} \mathrm{C}\left\{{ }^{1} \mathrm{H}\right\} \mathrm{NMR}\left(126 \mathrm{MHz}, \mathrm{CDCl}_{3}\right): \delta$ $159.3,145.5,137.8,135.3,133.3,129.1,128.7$, 126.8, 67.8, 35.2. HRMS (ESI/FTICR) $\mathrm{m} / z$ : $[\mathrm{M}+\mathrm{Na}]^{+}$calcd for $\mathrm{C}_{12} \mathrm{H}_{11} \mathrm{ClN}_{2} \mathrm{ONa} 257.04521$; found 257.04508 .

2-Bromo-6-phenethoxypyrazine (43). General Procedure D was applied using 2,6dibromopyrazine (127 mg, $0.534 \mathrm{mmol}, 1.0$ equiv.) and 2-phenylethan-1-ol (65.2 mg, $0.534 \mathrm{mmol}, 1.0$ equiv.). The reaction mixture was purified by FCC on silica using a gradient of EtOAc $(0 \%$ to $6 \%)$ in hexanes to give 43 as a colourless oil (122 mg, $0.436 \mathrm{mmol}$, $82 \%) .{ }^{1} \mathrm{H} \mathrm{NMR}\left(500 \mathrm{MHz}, \mathrm{CDCl}_{3}\right): \delta 8.21$ $(\mathrm{s}, 1 \mathrm{H}), 8.12(\mathrm{~s}, 1 \mathrm{H}), 7.35-7.20(\mathrm{~m}, 5 \mathrm{H})$, $4.55(\mathrm{t}, J=7.0 \mathrm{~Hz}, 2 \mathrm{H}), 3.09(\mathrm{t}, J=7.0$ $\mathrm{Hz}, 2 \mathrm{H}) .{ }^{13} \mathrm{C}\left\{{ }^{1} \mathrm{H}\right\} \mathrm{NMR}\left(126 \mathrm{MHz}, \mathrm{CDCl}_{3}\right)$ : $\delta$ 159.4, 138.3, 137.8, 136.5, 133.5, 129.1, 128.7, 126.8, 68.0, 35.2. HRMS (ESI/FTICR) $m / z: \quad[\mathrm{M}+\mathrm{Na}]^{+}$calcd for $\mathrm{C}_{12} \mathrm{H}_{11}{ }^{79} \mathrm{BrN}_{2} \mathrm{ONa}$ 300.99470 ; found 300.99465 .

2-Iodo-6-phenethoxypyrazine (44). General Procedure D was applied using 41 (108 mg, $0.325 \mathrm{mmol}, 1.0$ equiv.) and 2-phenylethan-1-ol (39.8 mg, $0.325 \mathrm{mmol}, 1.0$ equiv.). The reaction mixture was purified by FCC on silica using a gradient of EtOAc $(0 \%$ to $6 \%)$ in hexanes 
to give 44 as a colourless oil $(83.0 \mathrm{mg}, 0.254$ mmol, 78\%). ${ }^{1} \mathrm{H}$ NMR (500 $\left.\mathrm{MHz}, \mathrm{CDCl}_{3}\right)$ : $\delta 8.38(\mathrm{~s}, 1 \mathrm{H}), 8.11(\mathrm{~s}, 1 \mathrm{H}), 7.37-7.21(\mathrm{~m}$, $5 \mathrm{H}), 4.54(\mathrm{t}, J=7.0 \mathrm{~Hz}, 2 \mathrm{H}), 3.09(\mathrm{t}, J=7.0$ $\mathrm{Hz}, 2 \mathrm{H}) .{ }^{13} \mathrm{C}\left\{{ }^{1} \mathrm{H}\right\} \mathrm{NMR}\left(126 \mathrm{MHz}, \mathrm{CDCl}_{3}\right): \delta$ $159.5,144.2,137.8,133.7,129.1,128.7,126.8$, 112.7, 68.0, 35.2. HRMS (ESI/FTICR) $m / z$ : $[\mathrm{M}+\mathrm{Na}]^{+}$calcd for $\mathrm{C}_{12} \mathrm{H}_{11} \mathrm{IN}_{2} \mathrm{ONa} 348.98083$; found 348.98065 .

5-Chloro-[1,2,4]triazolo[4,3-a]pyrazine (45a ). General Procedure B was applied using S1 $(25.4 \mathrm{~g}, 176 \mathrm{mmol})$ to give $\mathbf{4 5 a}$ as a yellow solid (12.3 g, $79.8 \mathrm{mmol}, 45 \%) \cdot \operatorname{mp~} 169-171{ }^{\circ} \mathrm{C}$ (lit. ${ }^{8}$ 167-172 $\left.{ }^{\circ} \mathrm{C}\right) .{ }^{1} \mathrm{H}$ NMR $\left(500 \mathrm{MHz}, \mathrm{CDCl}_{3}\right)$ : $\delta 9.27(\mathrm{~s}, 1 \mathrm{H}), 9.04(\mathrm{~s}, 1 \mathrm{H}), 7.93(\mathrm{~s}, 1 \mathrm{H})$. ${ }^{13} \mathrm{C}\left\{{ }^{1} \mathrm{H}\right\} \mathrm{NMR}\left(126 \mathrm{MHz}, \mathrm{CDCl}_{3}\right): \delta$ 145.8, 141.9, 134.7, 128.3, 121.3. The spectroscopic data and melting point were in agreement with those in the literature. ${ }^{8} \mathrm{X}$-ray single crystal data can be found in the supporting information.

5-Chloro-3-(4-methoxyphenyl)-[1,2,4]triazolo/4,3-a]pyrazine (45b). General Procedure $\mathrm{C}$ was applied using S1 (1.01 g, $6.97 \mathrm{mmol}$, 1.0 equiv.) and 4-methoxybenzaldehyde (1.04 g, $7.66 \mathrm{mmol}, 1.1$ equiv.) to give $45 \mathrm{~b}$ as an off-white solid (1.34 g, $5.16 \mathrm{mmol}, 74 \%$ ). mp 145-147 ${ }^{\circ} \mathrm{C}$ (decomp.). ${ }^{1} \mathrm{H}$ NMR (200 MHz, $\left.\mathrm{CDCl}_{3}\right): \delta 9.31(\mathrm{~s}, 1 \mathrm{H}), 7.84(\mathrm{~s}, 1 \mathrm{H}), 7.63-$ $7.47(\mathrm{~m}, 2 \mathrm{H}), 7.11-6.95(\mathrm{~m}, 2 \mathrm{H}), 3.91(\mathrm{~s}$, $3 \mathrm{H}) .{ }^{13} \mathrm{C}\left\{{ }^{1} \mathrm{H}\right\}$ NMR $\left(126 \mathrm{MHz}\right.$, DMSO- $\left.d_{6}\right): \delta$ 160.8, 147.4, 146.9, 142.7, 132.8, 129.1, 121.8, 119.1, 113.1, 55.3. HRMS (ESI/FTICR) $m / z$ : $[\mathrm{M}+\mathrm{H}]^{+}$calcd for $\mathrm{C}_{12} \mathrm{H}_{10} \mathrm{ClN}_{4} \mathrm{O} 261.05377$; found 261.05348 .

5-Chloro-3-(4-nitrophenyl)-[1,2,4]triazolo-

[4,3-a]pyrazine (45c). General Procedure C was applied using S1 (1.06 g, $7.33 \mathrm{mmol}$, 1.0 equiv.) and 4-nitrobenzaldehyde (1.21 g, $8.07 \mathrm{mmol}, 1.1$ equiv.) to give $45 \mathrm{c}$ as an offwhite solid (1.91 g, $6.93 \mathrm{mmol}, 95 \%)$. mp 238-240 ${ }^{\circ} \mathrm{C}$ (decomp.). ${ }^{1} \mathrm{H}$ NMR (500 MHz, DMSO- $\left.d_{6}\right): \quad \delta 9.53(\mathrm{~s}, 1 \mathrm{H}), 8.41(\mathrm{~d}, J=$ $8.8 \mathrm{~Hz}, 2 \mathrm{H}), 8.15(\mathrm{~s}, 1 \mathrm{H}), 8.05(\mathrm{~d}, J=8.7$ $\mathrm{Hz}, 2 \mathrm{H}) .{ }^{13} \mathrm{C}\left\{{ }^{1} \mathrm{H}\right\}$ NMR $(126 \mathrm{MHz}$, DMSO$\left.d_{6}\right): \delta 148.6,147.2,145.8,142.7,133.7,132.9$, 129.4, 122.7, 121.9. HRMS (ESI/FTICR) $m / z$ : $[\mathrm{M}+\mathrm{H}]^{+}$calcd for $\mathrm{C}_{11} \mathrm{H}_{7} \mathrm{ClN}_{5} \mathrm{O}_{2}$ 276.02828; found 276.02784 .
5-Chloro-3-(2-methoxyphenyl)-[1,2,4/triazolo[4,3-a]pyrazine (45d). General Procedure C was applied using S1 (400 mg, $2.77 \mathrm{mmol}$, 1.0 equiv.) and 2-methoxybenzaldehyde (414 $\mathrm{mg}, 3.04 \mathrm{mmol}, 1.1$ equiv.) to give $45 \mathrm{~d}$ as an off-white solid (430 mg, $1.65 \mathrm{mmol}, 60 \%$ ); m.p. $142-145{ }^{\circ} \mathrm{C} .{ }^{1} \mathrm{H}$ NMR $(500 \mathrm{MHz}$, DMSO$\left.d_{6}\right): \delta 9.47(\mathrm{~s}, 1 \mathrm{H}), 8.08(\mathrm{~s}, 1 \mathrm{H}), 7.63(\mathrm{ddd}$, $J=8.3,7.5,1.7 \mathrm{~Hz}, 1 \mathrm{H}), 7.54(\mathrm{dd}, J=$ $7.5,1.7 \mathrm{~Hz}, 1 \mathrm{H}), 7.20(\mathrm{dd}, J=8.5,1.0 \mathrm{~Hz}$, $1 \mathrm{H}), 7.13(\mathrm{td}, \quad J=7.5,1.0 \mathrm{~Hz}, 1 \mathrm{H}), 3.73$ $(\mathrm{s}, 3 \mathrm{H}) ;{ }^{13} \mathrm{C}\left\{{ }^{1} \mathrm{H}\right\}$ NMR $(126 \mathrm{MHz}$, DMSO$\left.d_{6}\right) \delta 158.4,146.9,144.7,142.8,132.7,132.0$, 129.0, 121.8, 120.1, 116.3, 111.0, 55.4; HRMS $(\mathrm{ESI} / \mathrm{FTICR}+) \mathrm{m} / z:[\mathrm{M}+\mathrm{H}]^{+}$calcd for $\mathrm{C}_{12} \mathrm{H}_{10} \mathrm{ClN}_{4} \mathrm{O}$ 261.05377; found 261.05389.

5-Chloro-3-(2-nitrophenyl)-[1,2,4]triazolo-

[4,3-a/pyrazine (45e). General Procedure C was applied using S1 (1.04 g, $7.20 \mathrm{mmol}, 1.0$ equiv.) and 2-nitrobenzaldehyde (1.20 g, 7.92 mmol, 1.1 equiv.) to give $45 \mathrm{e}$ as a grey solid (1.74 g, $6.29 \mathrm{mmol}, 87 \%$ ). $\mathrm{mp} 224-228{ }^{\circ} \mathrm{C}$. ${ }^{1} \mathrm{H}$ NMR (500 MHz, DMSO- $\left.d_{6}\right): \delta 9.57(\mathrm{~s}, 1 \mathrm{H})$, $8.44(\mathrm{dd}, J=7.9,1.6 \mathrm{~Hz}, 1 \mathrm{H}), 8.17(\mathrm{~s}, 1 \mathrm{H}), 8.06$ $-7.95(\mathrm{~m}, 2 \mathrm{H}), 7.93$ (dd, $J=7.1,2.0 \mathrm{~Hz}, 1 \mathrm{H})$. ${ }^{13} \mathrm{C}\left\{{ }^{1} \mathrm{H}\right\}$ NMR $\left(126 \mathrm{MHz}, \mathrm{DMSO}-d_{6}\right): \delta 148.0$, 146.8, 143.8, 143.0, 134.5, 134.3, 132.9, 129.2, 125.0, 122.4, 121.4. HRMS (ESI/FTICR) $m / z$ : $[\mathrm{M}+\mathrm{Na}]^{+}$calcd for $\mathrm{C}_{11} \mathrm{H}_{6} \mathrm{ClN}_{5} \mathrm{O}_{2} \mathrm{Na} 298.01022$; found 298.01092.

5-Chloro-3-(3,5-di-tert-butylphenyl)-[1,2,4]triazolo/4,3-a]pyrazine (45f). General Procedure $\mathrm{C}$ was applied using $\mathbf{S 1}$ (1.05 g, $7.26 \mathrm{mmol}$, 1.0 equiv.) and 3,5-di-tert-butylbenzaldehyde (1.74 g, 7.99 mmol, 1.1 equiv.) to give $\mathbf{4 5 f}$ as a grey solid (1.68 g, $4.90 \mathrm{mmol}, 67 \%)$. mp 133-135 ${ }^{\circ} \mathrm{C} .{ }^{1} \mathrm{H}$ NMR (500 MHz, DMSO- $\left.d_{6}\right)$ : $\delta 9.46(\mathrm{~s}, 1 \mathrm{H}), 8.06(\mathrm{~s}, 1 \mathrm{H}), 7.61(\mathrm{t}, J=1.8$ $\mathrm{Hz}, 1 \mathrm{H}), 7.56(\mathrm{~d}, J=1.8 \mathrm{~Hz}, 2 \mathrm{H}), 1.34(\mathrm{~s}$, $18 \mathrm{H}) .{ }^{13} \mathrm{C}\left\{{ }^{1} \mathrm{H}\right\}$ NMR $\left(126 \mathrm{MHz}, \mathrm{DMSO}-d_{6}\right): \delta$ 149.6, 148.1, 147.0, 142.7, 129.2, 126.3, 125.8, 123.6, 121.8, 34.6, 31.1. HRMS (ESI/FTICR) $m / z:[\mathrm{M}+\mathrm{H}]^{+}$calcd for $\mathrm{C}_{19} \mathrm{H}_{24} \mathrm{ClN}_{4} 343.16840$; found 343.16865.

\section{3-(Anthracen-9-yl)-5-chloro-[1,2,4]triazolo-}

[4,3-a]pyrazine (45g). General Procedure C was applied using S1 (1.08 g, $7.47 \mathrm{mmol}, 1.0$ equiv.) and anthracene-9-carbaldehyde (1.69 g, $8.22 \mathrm{mmol}, 1.1$ equiv.) to give $\mathbf{4 5} \mathrm{g}$ as a bright 
yellow solid (1.62 g, $4.90 \mathrm{mmol}, 66 \%)$ mp $218-221{ }^{\circ} \mathrm{C} .{ }^{1} \mathrm{H}$ NMR $\left(500 \mathrm{MHz}, \mathrm{DMSO}-d_{6}\right): \delta$ $9.64(\mathrm{~s}, 1 \mathrm{H}), 8.96(\mathrm{~s}, 1 \mathrm{H}), 8.24(\mathrm{~d}, J=8.4 \mathrm{~Hz}$, $2 \mathrm{H}), 8.02(\mathrm{~s}, 1 \mathrm{H}), 7.58$ (ddd, $J=8.2,6.6,1.1$ $\mathrm{Hz}, 2 \mathrm{H}), 7.49$ (ddd, $J=8.8,6.5,1.2 \mathrm{~Hz}, 2 \mathrm{H}$ ), $7.34(\mathrm{dd}, J=8.7,1.1 \mathrm{~Hz}, 2 \mathrm{H}) .{ }^{13} \mathrm{C}\left\{{ }^{1} \mathrm{H}\right\} \mathrm{NMR}$ $\left(126 \mathrm{MHz}, \mathrm{DMSO}-d_{6}\right): \delta 147.8,144.0,143.4$, $132.4,130.6,130.3,129.2,128.6,127.5,125.8$, 125.5, 121.1, 120.5. HRMS (ESI/FTICR) $m / z$ : $[\mathrm{M}+\mathrm{H}]^{+}$calcd for $\mathrm{C}_{19} \mathrm{H}_{12} \mathrm{ClN}_{4}$ 331.07450; found 331.07449 .

5-Bromo-[1,2,4]triazolo[4,3-a]pyrazine (45h). General Procedure B was applied using S2 $(2.35 \mathrm{~g}, 12.4 \mathrm{mmol})$ to give $\mathbf{4 5 h}$ as an orange solid (1.75 g, $8.81 \mathrm{mmol}, 71 \%$ ). $\mathrm{mp} 167-170{ }^{\circ} \mathrm{C}$ (decomp.) (lit. $\left.{ }^{8} 214-217{ }^{\circ} \mathrm{C}\right) .{ }^{1} \mathrm{H}$ NMR (300 $\mathrm{MHz}$, DMSO- $\left.d_{6}\right): \quad \delta 9.62(\mathrm{~s}, 1 \mathrm{H}), 9.43(\mathrm{~s}$, $1 \mathrm{H}), 8.20(\mathrm{~s}, 1 \mathrm{H}) .{ }^{13} \mathrm{C}\left\{{ }^{1} \mathrm{H}\right\} \mathrm{NMR}(75 \mathrm{MHz}$, DMSO- $\left.d_{6}\right): \delta$ 145.3, 142.0, 137.5, 131.0, 109.9. HRMS (ESI/FTICR) $m / z:[\mathrm{M}+\mathrm{Na}]^{+}$calcd for $\mathrm{C}_{5} \mathrm{H}_{3}{ }^{79} \mathrm{BrN}_{4} \mathrm{Na} 220.94333$; found 220.94309 . The spectroscopic data were in agreement with the literature, but the melting point was significantly different.

5-Bromo-3-(4-methoxyphenyl)-[1,2,4]triazolo/4,3-a/pyrazine (45i). General Procedure $\mathrm{C}$ was applied using $\mathbf{S 2}$ (1.03 g, $5.46 \mathrm{mmol}, 1.0$ equiv.) and 4-methoxybenzaldehyde (0.818 g, $6.01 \mathrm{mmol}, 1.1$ equiv.) to give $45 \mathrm{i}$ as a pale brown solid (1.00 g, $3.27 \mathrm{mmol}, 60 \%)$. mp 156$157{ }^{\circ} \mathrm{C} .{ }^{1} \mathrm{H}$ NMR $\left(500 \mathrm{MHz}\right.$, DMSO- $\left.d_{6}\right): \delta 9.44$ $(\mathrm{s}, 1 \mathrm{H}), 8.10(\mathrm{~s}, 1 \mathrm{H}), 7.66-7.57(\mathrm{~m}, 2 \mathrm{H}), 7.13$ - $7.06(\mathrm{~m}, 2 \mathrm{H}), 3.86(\mathrm{~s}, 3 \mathrm{H}) .{ }^{13} \mathrm{C}\left\{{ }^{1} \mathrm{H}\right\} \mathrm{NMR}$ $\left(126 \mathrm{MHz}, \mathrm{DMSO}-d_{6}\right): \delta 160.8,148.1,146.6$, 143.0, 133.1, 132.7, 119.1, 113.1, 110.2, 55.3. HRMS (ESI/FTICR) $m / z:[\mathrm{M}+\mathrm{H}]^{+}$calcd for $\mathrm{C}_{12} \mathrm{H}_{10}{ }^{79} \mathrm{BrN}_{4} \mathrm{O}$ 305.00325; found 305.00303.

5-Bromo-3-(4-nitrophenyl)-[1,2,4]triazolo-

[4,3-a]pyrazine (45j). General Procedure C was applied using S2 (0.65 g, $3.4 \mathrm{mmol}$, 1.0 equiv.) and 4-nitrobenzaldehyde (0.57 g, 3.8 mmol, 1.1 equiv.) to give $\mathbf{4 5 j}$ as a yellow solid (0.93 g, $2.9 \mathrm{mmol}, 85 \%)$. mp 200-205 ${ }^{\circ} \mathrm{C}$ (decomp.). ${ }^{1} \mathrm{H}$ NMR (500 MHz, DMSO- $\left.d_{6}\right): \quad \delta 9.54(\mathrm{~s}, 1 \mathrm{H}), 8.41(\mathrm{~d}, J=$ $8.0 \mathrm{~Hz}, 2 \mathrm{H}), 8.20(\mathrm{~s}, 1 \mathrm{H}), 8.04(\mathrm{~d}, J=8.2$ $\mathrm{Hz}, 2 \mathrm{H}) .{ }^{13} \mathrm{C}\left\{{ }^{1} \mathrm{H}\right\}$ NMR $(126 \mathrm{MHz}$, DMSO$\left.d_{6}\right): \delta 148.7,146.9,146.4,143.0,133.9,133.2$, 133.0, 122.6, 110.4. HRMS (ESI/FTICR) $m / z$ :
$[\mathrm{M}+\mathrm{H}]^{+}$calcd for $\mathrm{C}_{11} \mathrm{H}_{7}{ }^{79} \mathrm{BrN}_{5} \mathrm{O}_{2}$ 319.97776; found 319.97811 .

5-Bromo-3-(2-methoxyphenyl)-[1,2,4]triazolo[4,3-a]pyrazine $(\mathbf{4 5 k})$. General Procedure C was applied using S2 (0.66 g, $3.5 \mathrm{mmol}, 1.0$ equiv.) and 2-methoxybenzaldehyde (0.52 g, $3.8 \mathrm{mmol}, 1.1$ equiv.) to give $45 \mathrm{k}$ as a white solid (0.75 g, $2.5 \mathrm{mmol}, 71 \%)$. mp $137-139{ }^{\circ} \mathrm{C}$. ${ }^{1} \mathrm{H} \mathrm{NMR}\left(500 \mathrm{MHz}, \mathrm{CDCl}_{3}\right): \delta 9.34(\mathrm{~s}, 1 \mathrm{H})$, $7.95(\mathrm{~s}, 1 \mathrm{H}), 7.58(\mathrm{ddd}, J=8.4,7.5,1.7 \mathrm{~Hz}$, $1 \mathrm{H}), 7.54(\mathrm{dd}, J=7.5,1.7 \mathrm{~Hz}, 1 \mathrm{H}), 7.11(\mathrm{td}, J$ $=7.5,1.0 \mathrm{~Hz}, 1 \mathrm{H}), 6.97(\mathrm{dd}, J=8.4,1.0 \mathrm{~Hz}$, $1 \mathrm{H}), 3.73(\mathrm{~s}, 3 \mathrm{H}) \cdot{ }^{13} \mathrm{C}\left\{{ }^{1} \mathrm{H}\right\}$ NMR $(126 \mathrm{MHz}$, $\left.\mathrm{CDCl}_{3}\right): \delta 159.2,147.1,146.6,143.4,133.1$, 133.0, 132.5, 120.5, 116.3, 110.5, 110.1, 55.4. HRMS (ESI/FTICR) $m / z:[\mathrm{M}+\mathrm{H}]^{+}$calcd for $\mathrm{C}_{12} \mathrm{H}_{10}{ }^{79} \mathrm{BrN}_{4} \mathrm{O}$ 305.00325; found 305.00359.

5-Bromo-3-(2-nitrophenyl)-[1,2,4]triazolo[4,3-a]pyrazine (451). General Procedure $\mathrm{C}$ was applied using S2 (0.62 g, $3.3 \mathrm{mmol}, 1.0$ equiv.) and 2-nitrobenzaldehyde (0.54 g, $3.6 \mathrm{mmol}, 1.1$ equiv.) to give $\mathbf{4 5 1}$ as a yellow solid $(0.84 \mathrm{~g}$, $2.6 \mathrm{mmol}, 81 \%) . \quad \mathrm{mp} 210-213{ }^{\circ} \mathrm{C} .{ }^{1} \mathrm{H}$ NMR $\left(200 \mathrm{MHz}, \mathrm{CDCl}_{3}\right): \delta 9.41(\mathrm{~s}, 1 \mathrm{H}), 8.50-8.36$ $(\mathrm{m}, 1 \mathrm{H}), 8.00(\mathrm{~s}, 1 \mathrm{H}), 7.94-7.80(\mathrm{~m}, 2 \mathrm{H})$, $7.72(\mathrm{~d}, J=6.6 \mathrm{~Hz}, 1 \mathrm{H}) .{ }^{13} \mathrm{C}\left\{{ }^{1} \mathrm{H}\right\} \mathrm{NMR}(126$ $\left.\mathrm{MHz}, \mathrm{DMSO}-d_{6}\right): \delta 148.1,146.4,144.6,143.2$, 134.6, 134.2, 132.9, 132.7, 124.9, 122.6, 109.9 . HRMS (ESI/FTICR) $m / z$ : $[\mathrm{M}+\mathrm{H}]^{+}$calcd for $\mathrm{C}_{11} \mathrm{H}_{7}{ }^{79} \mathrm{BrN}_{5} \mathrm{O}_{2}$ 319.97776; found 319.97803.

5-Iodo-[1,2,4]triazolo[4,3-a]pyrazine (45m). General Procedure B was applied using S3 (1.54 g, $6.52 \mathrm{mmol}, 1.0$ equiv.) to give $\mathbf{4 5 m}$ as a brown solid (1.08 g, $4.39 \mathrm{mmol}, 67 \%$, contains $0.5 \%$ DCM). mp $180-185{ }^{\circ} \mathrm{C}$ (decomp.). ${ }^{1} \mathrm{H}$ NMR $\left(500 \mathrm{MHz}, \mathrm{DMSO}-d_{6}\right): \delta 9.54(\mathrm{~s}, 1 \mathrm{H})$, $9.36(\mathrm{~s}, 1 \mathrm{H}), 8.24(\mathrm{~s}, 1 \mathrm{H}) .{ }^{13} \mathrm{C}\left\{{ }^{1} \mathrm{H}\right\} \mathrm{NMR}(126$ MHz, DMSO- $\left.d_{6}\right): \delta 144.4,142.2,140.2,137.7$, 83.9. HRMS (ESI/FTICR) $m / z:[\mathrm{M}+\mathrm{H}]^{+}$ calcd for $\mathrm{C}_{5} \mathrm{H}_{4} \mathrm{IN}_{4} 246.94752$; found 246.94745 .

5-Iodo-3-(4-methoxyphenyl)-[1,2,4]triazolo[4,3-a]pyrazine (45n). General Procedure C was applied using S3 (1.47 g, $6.21 \mathrm{mmol})$ and 4-methoxybenzaldehyde $(0.930 \mathrm{~g}, 6.83 \mathrm{mmol}$, 1.1 equiv.) to give $45 \mathrm{n}$ as an off-white solid (1.55 g, $4.39 \mathrm{mmol}, 71 \%$ ). $\mathrm{mp} 229-230{ }^{\circ} \mathrm{C}$ (decomp.). ${ }^{1} \mathrm{H}$ NMR $\left(500 \mathrm{MHz}, \mathrm{DMSO}-d_{6}\right): \delta 9.40$ (s, 1H), $8.22(\mathrm{~s}, 1 \mathrm{H}), 7.60-7.54(\mathrm{~m}, 2 \mathrm{H}), 7.15$ $7.09(\mathrm{~m}, 2 \mathrm{H}), 3.86(\mathrm{~s}, 3 \mathrm{H}) .{ }^{13} \mathrm{C}\left\{{ }^{1} \mathrm{H}\right\} \mathrm{NMR}$ 
$\left(126 \mathrm{MHz}, \mathrm{DMSO}-d_{6}\right): \delta 161.6,149.5,146.2$, $143.9,140.6,134.4,119.4,113.6,84.1,55.8$. HRMS (ESI/FTICR) $m / z:[\mathrm{M}+\mathrm{H}]^{+}$calcd for $\mathrm{C}_{12} \mathrm{H}_{10} \mathrm{IN}_{4} \mathrm{O}$ 352.98938; found 352.98910 .

5-Phenethoxy-[1,2,4]triazolo/4,3-a]pyrazine (46a). General Procedure D was applied using 45a (107 mg, $0.692 \mathrm{mmol})$ and 2-phenylethanol (84.5 mg, $0.692 \mathrm{mmol}, 1.0$ equiv.). The reaction mixture was purified by FCC on silica using a gradient of EtOAc (20 to 100\%) in hexanes to give $\mathbf{4 6 a}$ as an off-white solid $(125 \mathrm{mg}$, $0.520 \mathrm{mmol}, 75 \%) . \mathrm{mp} 143-146{ }^{\circ} \mathrm{C}$ (decomp.). ${ }^{1} \mathrm{H}$ NMR $\left(500 \mathrm{MHz}, \mathrm{DMSO}-d_{6}\right): \delta 9.38(\mathrm{~d}, J=$ $0.7 \mathrm{~Hz}, 1 \mathrm{H}), 9.02(\mathrm{t}, J=0.7 \mathrm{~Hz}, 1 \mathrm{H}), 7.63$ (s, $1 \mathrm{H}), 7.43-7.37(\mathrm{~m}, 2 \mathrm{H}), 7.35-7.28(\mathrm{~m}, 2 \mathrm{H})$, $7.26-7.19(\mathrm{~m}, 1 \mathrm{H}), 4.63(\mathrm{t}, J=6.7 \mathrm{~Hz}, 2 \mathrm{H})$, $3.19(\mathrm{t}, J=6.7 \mathrm{~Hz}, 2 \mathrm{H}) .{ }^{13} \mathrm{C}\left\{{ }^{1} \mathrm{H}\right\}$ NMR $(126$ $\left.\mathrm{MHz}, \mathrm{DMSO}-d_{6}\right): \delta 145.8,142.4,137.3,134.4$, 133.0, 129.2, 128.4, 126.5, 108.3, 71.3, 34.4. HRMS (ESI/FTICR) $m / z$ : $[\mathrm{M}+\mathrm{H}]^{+}$calcd for $\mathrm{C}_{13} \mathrm{H}_{13} \mathrm{~N}_{4} \mathrm{O}$ 241.10839; found 241.10813.

5-(Phenethylthio)-[1,2,4]triazolo[4,3-a]pyrazine (46b). General Procedure D was applied using 45a (105 mg, $0.681 \mathrm{mmol}, 1.0$ equiv.) and 2-phenylethane-1-thiol (94.2 mg, $0.681 \mathrm{mmol}$, 1.0 equiv.) to give $\mathbf{4 6} \mathrm{b}$ as an off-white solid (88.6 mg, $0.346 \mathrm{mmol}, 51 \%$ ). $\mathrm{mp} 108-110{ }^{\circ} \mathrm{C}$. ${ }^{1} \mathrm{H}$ NMR $\left(500 \mathrm{MHz}\right.$, DMSO- $\left.d_{6}\right): \delta 9.55(\mathrm{~d}, J$ $=0.8 \mathrm{~Hz}, 1 \mathrm{H}), 9.33(\mathrm{~s}, 1 \mathrm{H}), 8.03(\mathrm{~s}, 1 \mathrm{H}), 7.27$ $-7.20(\mathrm{~m}, 4 \mathrm{H}), 7.20-7.12(\mathrm{~m}, 1 \mathrm{H}), 3.48(\mathrm{dd}$, $J=7.9,7.0 \mathrm{~Hz}, 2 \mathrm{H}), 2.95(\mathrm{t}, J=7.4 \mathrm{~Hz}, 2 \mathrm{H})$. ${ }^{13} \mathrm{C}\left\{{ }^{1} \mathrm{H}\right\}$ NMR $\left(126 \mathrm{MHz}\right.$, DMSO- $\left.d_{6}\right): \delta$ 144.6, 141.5, 139.0, 136.1, 131.5, 128.6, 128.2, 126.4, 126.1, 34.8, 33.5. HRMS (ESI/FTICR) $m / z$ : $[\mathrm{M}+\mathrm{H}]^{+}$calcd for $\mathrm{C}_{13} \mathrm{H}_{13} \mathrm{~N}_{4} \mathrm{~S} 257.08554$; found 257.08534 .

3-(4-Methoxyphenyl)-5-phenethoxy-[1,2,4]triazolo/4,3-a]pyrazine (46c). Preparation 1: General Procedure D was applied using 45b (103 mg, 0.395 mmol, 1.0 equiv.) and 2phenylethan-1-ol (48.3 mg, $0.395 \mathrm{mmol}, 1.0$ equiv.) to give $46 \mathrm{c}$ as a yellow solid $(95.1 \mathrm{mg}$, $0.275 \mathrm{mmol}, 69 \%$ ). Preparation 2: General Procedure D was applied using 45i (122 mg, 0.400 mmol, 1.0 equiv.) and 2-phenylethan-1-ol (48.9 $\mathrm{mg}, 0.400 \mathrm{mmol}, 1.0$ equiv.). Fractions corresponding to the second peak were evaporated to give 46c (first run: $45.4 \mathrm{mg}, 0.127 \mathrm{mmol}$, $33 \%$, second run: $43.0 \mathrm{mg}, 0.124 \mathrm{mmol}, 31 \%$, average yield is $32 \%$ ). $\operatorname{mp~} 162-163{ }^{\circ} \mathrm{C} .{ }^{1} \mathrm{H}$ NMR $\left(500 \mathrm{MHz}, \mathrm{DMSO}-d_{6}\right): \delta 9.00(\mathrm{~s}, 1 \mathrm{H}), 7.67-$ $7.60(\mathrm{~m}, 2 \mathrm{H}), 7.55(\mathrm{~s}, 1 \mathrm{H}), 7.22-7.12(\mathrm{~m}, 3 \mathrm{H})$, $7.07-7.01(\mathrm{~m}, 2 \mathrm{H}), 6.96-6.88(\mathrm{~m}, 2 \mathrm{H}), 4.48$ $(\mathrm{t}, J=6.4 \mathrm{~Hz}, 2 \mathrm{H}), 3.83(\mathrm{~s}, 3 \mathrm{H}), 2.89(\mathrm{t}, J=$ $6.4 \mathrm{~Hz}, 2 \mathrm{H}) .{ }^{13} \mathrm{C}\left\{{ }^{1} \mathrm{H}\right\}$ NMR $(126 \mathrm{MHz}, \mathrm{DMSO}-$ $\left.d_{6}\right): \delta 160.4,147.3,146.3,143.9,137.4,135.0$, 132.2, 128.7, 128.2, 126.3, 120.0, 113.0, 108.6, 71.2, 55.3, 33.9. HRMS (ESI/FTICR) $m / z$ : $[\mathrm{M}+\mathrm{H}]^{+}$calcd for $\mathrm{C}_{20} \mathrm{H}_{19} \mathrm{~N}_{4} \mathrm{O}_{2}$ 347.15025; found 347.14983.

3-(4-Methoxyphenyl)-5-(phenethylthio) [1,2,4]triazolo[4,3-a]pyrazine (46d). General Procedure D was applied using 45b (100 mg, 0.384 mmol, 1.0 equiv.) and 2-phenylethane-1-thiol (53.0 mg, $0.384 \mathrm{mmol}, 1.0$ equiv.). The reaction mixture was purified by FCC on silica using a gradient of $\mathrm{MeOH}(0 \%$ to $10 \%)$ in DCM, fractions corresponding to the second peak were evaporated to give $\mathbf{4 6 d}$ as a yellow solid (11.3 mg, $0.0312 \mathrm{mmol}, 8 \%$ ). $\mathrm{mp} 202$ $205{ }^{\circ} \mathrm{C} .{ }^{1} \mathrm{H}$ NMR $\left(500 \mathrm{MHz}, \mathrm{CD}_{3} \mathrm{CN}\right): \delta 9.12$ $(\mathrm{s}, 1 \mathrm{H}), 7.78(\mathrm{~s}, 1 \mathrm{H}), 7.59-7.51(\mathrm{~m}, 2 \mathrm{H}), 7.27$ $7.19(\mathrm{~m}, 2 \mathrm{H}), 7.21-7.14(\mathrm{~m}, 1 \mathrm{H}), 7.09-$ $6.99(\mathrm{~m}, 4 \mathrm{H}), 3.89(\mathrm{~s}, 3 \mathrm{H}), 2.94(\mathrm{t}, J=7.3 \mathrm{~Hz}$, $2 \mathrm{H}), 2.72(\mathrm{t}, J=7.3 \mathrm{~Hz}, 2 \mathrm{H}) .{ }^{13} \mathrm{C}\left\{{ }^{1} \mathrm{H}\right\} \mathrm{NMR}$ $\left(126 \mathrm{MHz}, \mathrm{CD}_{3} \mathrm{CN}\right): \delta 162.5,149.2,147.5$, $142.8, \quad 140.2,134.1, \quad 132.3,129.59,129.56$, $129.4,127.5,120.7,113.9,56.2,36.3,35.1$. HRMS (ESI/FTICR) $m / z: \quad[\mathrm{M}+\mathrm{H}]^{+}$calcd for $\mathrm{C}_{20} \mathrm{H}_{19} \mathrm{~N}_{4} \mathrm{OS} 363.12741$; found 363.12700.

3-(4-Nitrophenyl)-5-phenethoxy-[1,2,4]triazolo/4,3-a]pyrazine (46e). Preparation 1: General Procedure D was applied using 45c (104 mg, $0.377 \mathrm{mmol}, 1.0$ equiv.) and 2phenylethan-1-ol (46.1 mg, $0.377 \mathrm{mmol}, 1.0$ equiv.). Fraction corresponding to the second peak were evaporated to give $46 \mathrm{e}$ as a yellow solid (105 mg, $0.290 \mathrm{mmol}, 77 \%$ ). Preparation 2: General Procedure D was applied using 45j (128 mg, $0.400 \mathrm{mmol}, 1.0$ equiv.) and 2-phenylethan-1-ol (48.8 mg, $0.400 \mathrm{mmol}$, 1.0 equiv.) to give $46 \mathrm{e}$ (first run: $85.6 \mathrm{mg}$, $0.237 \mathrm{mmol}, 59 \%$, second run: $87.8 \mathrm{mg}, 0.243$ mmol, 61\%, average yield is 60\%). m.p.168 $170{ }^{\circ} \mathrm{C} .{ }^{1} \mathrm{H}$ NMR $\left(500 \mathrm{MHz}\right.$, DMSO- $\left.d_{6}\right): \delta 9.11$ $(\mathrm{s}, 1 \mathrm{H}), 8.29-8.22(\mathrm{~m}, 2 \mathrm{H}), 7.99-7.93(\mathrm{~m}$, $2 \mathrm{H}), 7.70(\mathrm{~s}, 1 \mathrm{H}), 7.15(\mathrm{dd}, J=5.0,1.9 \mathrm{~Hz}$, $3 \mathrm{H}), 6.95(\mathrm{dd}, J=6.6,2.9 \mathrm{~Hz}, 2 \mathrm{H}), 4.58(\mathrm{t}$, 
$J=6.4 \mathrm{~Hz}, 2 \mathrm{H}), 2.93(\mathrm{t}, J=6.4 \mathrm{~Hz}, 2 \mathrm{H})$. ${ }^{13} \mathrm{C}\left\{{ }^{1} \mathrm{H}\right\}$ NMR $\left(126 \mathrm{MHz}\right.$, DMSO- $\left.d_{6}\right): \delta 147.9$, $147.6,144.6,143.8,137.1,134.9,134.1,131.9$, 128.4, 128.1, 126.3, 122.6, 109.3, 70.9, 33.5. HRMS (ESI/FTICR) $m / z:[\mathrm{M}+\mathrm{H}]^{+}$calcd for $\mathrm{C}_{19} \mathrm{H}_{16} \mathrm{~N}_{5} \mathrm{O}_{3} 362.12477$; found 362.12448 .

3-(2-Methoxyphenyl)-5-phenethoxy-[1,2,4]triazolo/4,3-alpyrazine (46f). General Procedure D was applied using 45k (122 mg, 0.400 mmol, 1.0 equiv.) and 2-phenylethan-1-ol (48.8 $\mathrm{mg}, 0.400 \mathrm{mmol}, 1.0$ equiv.). Fractions corresponding to the second peak were combined and evaporated to give $\mathbf{4 6 f}$ as a yellow solid (first run: $36.1 \mathrm{mg}, 0.104 \mathrm{mmol}, 26 \%$, second run: $34.2 \mathrm{mg}, 0.100 \mathrm{mmol}, 25 \%$, average yield is $26 \%$ ). mp $147-150{ }^{\circ} \mathrm{C} \cdot{ }^{1} \mathrm{H} \mathrm{NMR}(500 \mathrm{MHz}$, DMSO- $\left.d_{6}\right): \delta 9.02(\mathrm{~s}, 1 \mathrm{H}), 7.62(\mathrm{ddd}, J=$ 8.3, 7.5, $1.8 \mathrm{~Hz}, 1 \mathrm{H}), 7.53(\mathrm{~s}, 1 \mathrm{H}), 7.50$ (dd, $J=7.5,1.8 \mathrm{~Hz}, 1 \mathrm{H}), 7.22(\mathrm{dd}, J=8.4,1.0$ $\mathrm{Hz}, 1 \mathrm{H}), 7.20-7.10(\mathrm{~m}, 4 \mathrm{H}), 6.79-6.73(\mathrm{~m}$, $2 \mathrm{H}), 4.37$ (s, 2H), $3.72(\mathrm{~s}, 3 \mathrm{H}), 2.65(\mathrm{~s}, 2 \mathrm{H})$. ${ }^{13} \mathrm{C}\left\{{ }^{1} \mathrm{H}\right\}$ NMR $\left(126 \mathrm{MHz}, \mathrm{DMSO}-d_{6}\right): \delta 158.2$, $147.1,143.9,143.2,137.2,135.1,132.0,131.4$, $128.8,128.2$, 126.3, 119.9, 117.4, 110.9, 108.8, 71.4, 55.4, 34.1. HRMS (ESI/FTICR) $m / z$ : $[\mathrm{M}+\mathrm{H}]^{+}$calcd for $\mathrm{C}_{20} \mathrm{H}_{19} \mathrm{~N}_{4} \mathrm{O}_{2}$ 347.15025; found 347.15041 .

\section{3-(2-Nitrophenyl)-5-phenethoxy-[1,2,4]tria-}

zolo/4,3-alpyrazine (46g). Preparation 1: General Procedure D was applied using 45e (110 mg, 0.399 mmol, 1.0 equiv.) and 2phenylethan-1-ol (48.8 mg, $0.399 \mathrm{mmol}, 1.0$ equiv.). The reaction mixture was purified by FCC on silica using a gradient of EtOAc $(30 \%$ to $100 \%)$ in hexanes, then $\mathrm{MeOH}(0 \%$ to $5 \%)$ in EtOAc to give $46 \mathrm{~g}$ as a yellow solid (first run: $123 \mathrm{mg}, 0.341 \mathrm{mmol}, 86 \%$, second run: $113 \mathrm{mg}, 0.313 \mathrm{mmol}, 79 \%$, average yield is 83\%). Preparation 2: General Procedure D was applied using $45 \mathrm{l}$ (128 mg, $0.400 \mathrm{mmol}$, 1.0 equiv.) and 2-phenylethan-1-ol (48.8 mg, $0.400 \mathrm{mmol}, 1.0$ equiv.) to give $46 \mathrm{~g}$ (first run: $108 \mathrm{mg}, 0.299 \mathrm{mmol}, 75 \%$, second run: $111 \mathrm{mg}$, $0.307 \mathrm{mmol}, 77 \%$, average yield is $76 \%) \mathrm{mp}$ 178-181 ${ }^{\circ} \mathrm{C}$ (decomp.). ${ }^{1} \mathrm{H}$ NMR (500 MHz, DMSO- $\left.d_{6}\right): \delta 9.13(\mathrm{~s}, 1 \mathrm{H}), 8.37(\mathrm{dd}, J=8.1$, $1.4 \mathrm{~Hz}, 1 \mathrm{H}), 7.96(\mathrm{td}, J=7.5,1.4 \mathrm{~Hz}, 1 \mathrm{H})$, $7.90(\mathrm{td}, J=7.8,1.6 \mathrm{~Hz}, 1 \mathrm{H}), 7.85(\mathrm{dd}, J=$ 7.5, 1.6 Hz, 1H), $7.63(\mathrm{~s}, 1 \mathrm{H}), 7.18-7.11$ (m,
$3 \mathrm{H}), 6.83-6.76(\mathrm{~m}, 2 \mathrm{H}), 4.38(\mathrm{t}, J=6.5 \mathrm{~Hz}$, $2 \mathrm{H}), 2.68(\mathrm{t}, J=6.4 \mathrm{~Hz}, 2 \mathrm{H}) .{ }^{13} \mathrm{C}\left\{{ }^{1} \mathrm{H}\right\} \mathrm{NMR}$ $\left(126 \mathrm{MHz}, \mathrm{DMSO}-d_{6}\right): \delta 147.7,147.0,143.1$, $142.5,136.7,135.3,134.0,133.8,131.9,128.4$, 128.2, 126.4, 124.7, 123.1, 109.1, 71.2, 33.6. HRMS (ESI/FTICR) $m / z:[\mathrm{M}+\mathrm{H}]^{+}$calcd for $\mathrm{C}_{19} \mathrm{H}_{16} \mathrm{~N}_{5} \mathrm{O}_{3} 362.12477$; found 362.12469 .

3-(3,5-Di-tert-butylphenyl)-5-phenethoxy-

[1,2,4]triazolo[4,3-a]pyrazine (46h). General Procedure D was applied using 45f $(137 \mathrm{mg}$, $0.400 \mathrm{mmol}, 1.0$ equiv.) and 2-phenylethan-1-ol (48.8 mg, $0.400 \mathrm{mmol}, 1.0$ equiv.). Fractions corresponding to the second peak were combined and evaporated to give $46 \mathrm{~h}$ as a yellow solid (first run: $139 \mathrm{mg}, 0.325 \mathrm{mmol}, 81 \%$, second run: $140 \mathrm{mg}, 0.326 \mathrm{mmol}, 82 \%$, average yield is $82 \%$ ). $\operatorname{mp~} 175-177{ }^{\circ} \mathrm{C} .{ }^{1} \mathrm{H}$ NMR $\left(500 \mathrm{MHz}, \mathrm{DMSO}-d_{6}\right): \delta 9.01(\mathrm{~s}, 1 \mathrm{H}), 7.65(\mathrm{t}$, $J=1.9 \mathrm{~Hz}, 1 \mathrm{H}), 7.57(\mathrm{~d}, J=1.8 \mathrm{~Hz}, 2 \mathrm{H})$, $7.54(\mathrm{~s}, 1 \mathrm{H}), 7.16-7.01(\mathrm{~m}, 3 \mathrm{H}), 6.75-6.68$ $(\mathrm{m}, 2 \mathrm{H}), 4.43(\mathrm{t}, J=6.3 \mathrm{~Hz}, 2 \mathrm{H}), 2.76(\mathrm{t}, J$ $=6.2 \mathrm{~Hz}, 2 \mathrm{H}), 1.37(\mathrm{~s}, 18 \mathrm{H}) \cdot{ }^{13} \mathrm{C}\left\{{ }^{1} \mathrm{H}\right\} \mathrm{NMR}$ $\left(126 \mathrm{MHz}, \mathrm{DMSO}-d_{6}\right): \delta 149.6,147.2,147.0$, $143.9,137.4,135.0,128.6,128.1,127.4,126.3$, 124.7, 123.7, 108.8, 71.4, 34.7, 34.1, 31.3. HRMS (ESI/FTICR) $m / z:[\mathrm{M}+\mathrm{Na}]^{+}$calcd for $\mathrm{C}_{27} \mathrm{H}_{32} \mathrm{~N}_{4} \mathrm{ONa} 451.24683$; found 451.24708.

3-(Anthracen-9-yl)-5-phenethoxy-[1,2,4]triazolo/4,3-alpyrazine (46i). General Procedure D was applied using $45 \mathrm{~g}$ (132 $\mathrm{mg}$, $0.399 \mathrm{mmol}$, 1.0 equiv.) and 2-phenylethan-1-ol (48.8 mg, $0.399 \mathrm{mmol}, 1.0$ equiv.). Fractions corresponding to the second peak were combined and evaporated to give $\mathbf{4 6} \mathbf{i}$ as a yellow solid (first run: $110 \mathrm{mg}, 0.264 \mathrm{mmol}, 66 \%$, second run: $107 \mathrm{mg}, 0.258 \mathrm{mmol}$, 65\%, average yield is $66 \%) . \quad m p ~ 207-211{ }^{\circ} \mathrm{C} .{ }^{1} \mathrm{H}$ NMR $(500 \mathrm{MHz}$, DMSO- $\left.d_{6}\right): \delta 9.22(\mathrm{~s}, 1 \mathrm{H}), 8.95(\mathrm{~s}, 1 \mathrm{H}), 8.27$ $(\mathrm{d}, J=8.5 \mathrm{~Hz}, 2 \mathrm{H}), 7.62$ (ddd, $J=8.3,6.6$, $1.1 \mathrm{~Hz}, 2 \mathrm{H}), 7.56-7.47(\mathrm{~m}, 3 \mathrm{H}), 7.39$ (dd, J $=8.7,1.1 \mathrm{~Hz}, 2 \mathrm{H}), 6.96-6.89(\mathrm{~m}, 1 \mathrm{H}), 6.78$ - $6.70(\mathrm{~m}, 2 \mathrm{H}), 6.08-6.03(\mathrm{~m}, 2 \mathrm{H}), 3.94(\mathrm{t}$, $J=6.2 \mathrm{~Hz}, 2 \mathrm{H}), 1.57(\mathrm{t}, J=6.2 \mathrm{~Hz}, 2 \mathrm{H})$. ${ }^{13} \mathrm{C}\left\{{ }^{1} \mathrm{H}\right\}$ NMR $\left(126 \mathrm{MHz}\right.$, DMSO- $\left.d_{6}\right): \delta 147.7$, $143.6,142.4,136.7,135.7,131.8,130.5,129.8$, $128.5,128.2,127.7,127.1,126.0,125.6,125.5$, 121.8, 109.2, 71.4, 33.3. HRMS (ESI/FTICR) $m / z:[\mathrm{M}+\mathrm{H}]^{+}$calcd for $\mathrm{C}_{27} \mathrm{H}_{21} \mathrm{~N}_{4} \mathrm{O} 417.17099$; found 417.17134 . 
3-(4-Methoxyphenyl)-8-phenethoxy-[1,2,4]triazolo/4,3-a]pyrazine (47a). Preparation 1: General Procedure D was applied using 45n (132 mg, $0.375 \mathrm{mmol}, 1.0$ equiv.) and 2phenylethan-1-ol (45.8 mg, $0.375 \mathrm{mmol}, 1.0$ equiv.). Fraction corresponding to the first peak were evaporated to give $47 \mathbf{a}$ as an offwhite solid (70.0 mg, $0.202 \mathrm{mmol}$, 54\%). Preparation 2: isolated from the same reaction as for 46c preparation 2. Fractions corresponding to the first peak were evaporated to give $\mathbf{4 7 a}$ (first run: $13.0 \mathrm{mg}, 0.0375$ mmol, 9\%, second run: $15.5 \mathrm{mg}, 0.0447 \mathrm{mmol}$, $11 \%$, average yield is $10 \%) . \operatorname{mp} 208-211{ }^{\circ} \mathrm{C}$. ${ }^{1} \mathrm{H}$ NMR $\left(500 \mathrm{MHz}, \mathrm{DMSO}-d_{6}\right): \delta 8.17-8.12$ $(\mathrm{m}, 1 \mathrm{H}), 7.88-7.81(\mathrm{~m}, 2 \mathrm{H}), 7.47-7.42$ $(\mathrm{m}, 1 \mathrm{H}), 7.40-7.29(\mathrm{~m}, 4 \mathrm{H}), 7.27-7.23$ $(\mathrm{m}, 1 \mathrm{H}), 7.22-7.15(\mathrm{~m}, 2 \mathrm{H}), 4.76(\mathrm{t}, J=$ $6.8 \mathrm{~Hz}, 2 \mathrm{H}), 3.87(\mathrm{~s}, 2 \mathrm{H}), 3.18(\mathrm{t}, J=6.9$ $\mathrm{Hz}, 2 \mathrm{H}) .{ }^{13} \mathrm{C}\left\{{ }^{1} \mathrm{H}\right\}$ NMR $(126 \mathrm{MHz}$, DMSO$\left.d_{6}\right): \delta 160.8,153.2,147.9,139.5,138.0,129.7$, $128.9,128.4,127.2,126.4,118.1,114.8,112.1$, 67.3, 55.4, 34.2. HRMS (ESI/FTICR) $m / z$ : $[\mathrm{M}+\mathrm{H}]^{+}$calcd for $\mathrm{C}_{20} \mathrm{H}_{19} \mathrm{~N}_{4} \mathrm{O}_{2}$ 347.15025; found 347.14994 .

3-(4-Methoxyphenyl)-8-(phenethylthio) [1,2,4]triazolo[4,3-a]pyrazine (47b). Preparation 1: General Procedure D was applied using 45i (110 mg, $0.360 \mathrm{mmol}$ ) and 2-phenylethane-1thiol (50.0 mg, $0.360 \mathrm{mmol}, 1.0$ equiv.) to give $\mathbf{4 7 b}$ as a yellow solid (122 mg, $0.337 \mathrm{mmol}$, 93\%). Preparation 2: General Procedure D was applied using 45n (108 mg, $0.307 \mathrm{mmol}, 1.0$ equiv.) and 2-phenylethane-1-thiol $(43.0 \mathrm{mg}$, $0.307 \mathrm{mmol}, 1.0$ equiv.) to give $47 \mathrm{~b}(14.0 \mathrm{mg}$, $0.0390 \mathrm{mmol}, 13 \%$ ). Preparation 3: isolated from the same reaction as for $46 d$. Fractions corresponding to the first peak were evaporated to give $47 \mathrm{~b}$ (116 mg, $0.319 \mathrm{mmol}, 83 \%$ ). $\mathrm{mp}$ 192-194 ${ }^{\circ} \mathrm{C} .{ }^{1} \mathrm{H}$ NMR $\left(200 \mathrm{MHz}, \mathrm{CDCl}_{3}\right): \delta$ $7.85(\mathrm{~d}, J=4.8 \mathrm{~Hz}, 1 \mathrm{H}), 7.77(\mathrm{~d}, J=8.7$ $\mathrm{Hz}, 2 \mathrm{H}), 7.69(\mathrm{~d}, J=4.8 \mathrm{~Hz}, 1 \mathrm{H}), 7.32(\mathrm{~d}$, $J=4.3 \mathrm{~Hz}, 4 \mathrm{H}), 7.11(\mathrm{~d}, J=8.8 \mathrm{~Hz}, 2 \mathrm{H})$, $3.91(\mathrm{~s}, 3 \mathrm{H}), 3.69-3.52(\mathrm{~m}, 2 \mathrm{H}), 3.20-3.02$ $(\mathrm{m}, 2 \mathrm{H}) .{ }^{13} \mathrm{C}\left\{{ }^{1} \mathrm{H}\right\}$ NMR $(126 \mathrm{MHz}$, DMSO$\left.d_{6}\right): \delta 160.9,152.9,147.6,143.7,140.0,129.8$, $129.3,128.6,128.4,126.4,117.9,114.8,113.2$, 55.5, 34.5, 29.4. HRMS (ESI/FTICR) $m / z$ : $[\mathrm{M}+\mathrm{H}]^{+}$calcd for $\mathrm{C}_{20} \mathrm{H}_{19} \mathrm{~N}_{4} \mathrm{OS}$ 363.12741; found 363.12676 .

3-(4-Methoxyphenyl)-N-phenethyl-[1,2,4]-triazolo/4,3-a]pyrazin-8-amine (47c). Preparation 1: General Procedure E was applied using 45b (106 mg, $0.407 \mathrm{mmol}, 1.0$ equiv.) and 2phenylethan-1-amine (148 mg, $1.22 \mathrm{mmol}, 3.0$ equiv.) to give 47c as a yellow solid (126 mg, 0.365 mmol, 90\%). Preparation 2: General Procedure E was applied using 45i (101 mg, $0.331 \mathrm{mmol}, 1.0$ equiv.) and 2-phenylethan1-amine (120 mg, $0.993 \mathrm{mmol}, 3.0$ equiv.) to give 47c (75.0 mg, $0.217 \mathrm{mmol}, 66 \%$ ). Preparation 3: General Procedure E was applied using 45n (341 mg, $0.968 \mathrm{mmol}$ ) and 2-phenylethan1-amine (350 mg, $2.91 \mathrm{mmol}, 3.0$ equiv.) to give 47c (195 mg, $0.564 \mathrm{mmol}, 58 \%$ ). $\mathrm{mp}$ $193-196{ }^{\circ} \mathrm{C} .{ }^{1} \mathrm{H}$ NMR (500 MHz, DMSO- $\left.d_{6}\right)$ : $\delta 8.23(\mathrm{t}, J=5.8 \mathrm{~Hz}, 1 \mathrm{H}), 7.86-7.80(\mathrm{~m}$, $2 \mathrm{H}), 7.69(\mathrm{~d}, J=4.8 \mathrm{~Hz}, 1 \mathrm{H}), 7.34(\mathrm{~d}, J=$ $4.9 \mathrm{~Hz}, 1 \mathrm{H}), 7.32-7.25(\mathrm{~m}, 4 \mathrm{H}), 7.21(\mathrm{dd}$, $J=6.8,2.1 \mathrm{~Hz}, 1 \mathrm{H}), 7.19-7.15(\mathrm{~m}, 2 \mathrm{H})$, $3.86(\mathrm{~s}, 3 \mathrm{H}), 3.74(\mathrm{q}, J=6.9 \mathrm{~Hz}, 2 \mathrm{H}), 2.98$ $(\mathrm{t}, J=7.5 \mathrm{~Hz}, 2 \mathrm{H}) .{ }^{13} \mathrm{C}\left\{{ }^{1} \mathrm{H}\right\} \mathrm{NMR}(126 \mathrm{MHz}$, DMSO- $\left.d_{6}\right): \delta 160.6,147.9,147.6,139.5,139.4$, $129.9,129.5,128.7,128.3,126.0,118.5,114.7$, 105.9, 55.4, 41.6, 34.5. HRMS (ESI/FTICR) $m / z:[\mathrm{M}+\mathrm{H}]^{+}$calcd for $\mathrm{C}_{20} \mathrm{H}_{20} \mathrm{~N}_{5} \mathrm{O} 346.16624$; found 346.16572 .

\section{3-(3,5-Di-tert-butylphenyl)-8-phenethoxy-}

[1,2,4]tria-zolo[4,3-a]pyrazine (47d). Isolated from the same reaction as for $\mathbf{4 6 h}$. Fractions corresponding to the first peak were combined and evaporated to give $\mathbf{4 7 d}$ as a yellow sticky solid (first run: $5.0 \mathrm{mg}, 11.6 \mu \mathrm{mol}, 3 \%$, second run: $5.00 \mathrm{mg}, 11.6 \mu \mathrm{mol}, 3 \%$, average yield is $3 \%) .{ }^{1} \mathrm{H} \mathrm{NMR}\left(500 \mathrm{MHz}, \mathrm{CDCl}_{3}\right): \delta 7.76(\mathrm{~d}, J$ $=4.8 \mathrm{~Hz}, 1 \mathrm{H}), 7.65-7.60(\mathrm{~m}, 3 \mathrm{H}), 7.39-7.27$ $(\mathrm{m}, 5 \mathrm{H}), 7.24(\mathrm{t}, J=7.2 \mathrm{~Hz}, 1 \mathrm{H}), 4.81(\mathrm{t}, J$ $=7.5 \mathrm{~Hz}, 2 \mathrm{H}), 3.28(\mathrm{t}, J=7.5 \mathrm{~Hz}, 2 \mathrm{H}), 1.39$ $(\mathrm{s}, 18 \mathrm{H}) \cdot{ }^{13} \mathrm{C}\left\{{ }^{1} \mathrm{H}\right\} \mathrm{NMR}\left(126 \mathrm{MHz}, \mathrm{CDCl}_{3}\right): \delta$ $154.4,152.3,149.8,140.5,137.7,129.3,128.7$, $128.0,126.8,125.4,125.1,122.8,110.8,68.3$, 35.3, 35.2, 31.5. HRMS (ESI/FTICR) $m / z$ : $[\mathrm{M}+\mathrm{Na}]^{+}$calcd for $\mathrm{C}_{27} \mathrm{H}_{32} \mathrm{~N}_{4} \mathrm{ONa} 451.24683$; found 451.24714 .

3-(Anthracen-9-yl)-8-phenethoxy-[1,2,4]triazolo[4,3-a]pyrazine (47e). Isolated from the same reaction as for 46i. Fractions corresponding to the first peak were combined and 
evaporated to give 47e as a yellow solid (first run: $2.50 \mathrm{mg}, 6.00 \mu \mathrm{mol}, 2 \%$, second run: 3.00 $\mathrm{mg}, 7.20 \mu \mathrm{mol}, 2 \%$, average yield is $2 \%$ ). $\mathrm{mp}$ 175-180 ${ }^{\circ} \mathrm{C}$ (decomp.). ${ }^{1} \mathrm{H}$ NMR (500 MHz, DMSO- $\left.d_{6}\right): \delta 9.00(\mathrm{~s}, 1 \mathrm{H}), 8.28(\mathrm{~d}, J=8.5$ $\mathrm{Hz}, 2 \mathrm{H}), 7.74-7.68(\mathrm{~m}, 1 \mathrm{H}), 7.61$ (ddd, $J=$ 8.3, 6.6, $1.1 \mathrm{~Hz}, 2 \mathrm{H}), 7.51$ (ddd, $J=8.9,6.6$, $1.3 \mathrm{~Hz}, 2 \mathrm{H}), 7.47-7.42(\mathrm{~m}, 2 \mathrm{H}), 7.41-7.34$ $(\mathrm{m}, 4 \mathrm{H}), 7.32(\mathrm{~d}, J=4.8 \mathrm{~Hz}, 1 \mathrm{H}), 7.30-7.23$ $(\mathrm{m}, 2 \mathrm{H}), 4.83(\mathrm{t}, J=6.8 \mathrm{~Hz}, 2 \mathrm{H}), 3.25(\mathrm{t}$, $J=6.8 \mathrm{~Hz}, 2 \mathrm{H}) .{ }^{13} \mathrm{C}\left\{{ }^{1} \mathrm{H}\right\} \mathrm{NMR}(126 \mathrm{MHz}$, DMSO- $\left.d_{6}\right): \delta 153.4,145.4,141.8,141.4,140.0$, 138.1, 131.0, 130.83, 130.79, 129.3, 129.0, 128.9, 128.4, 127.8, 127.5, 126.5, 125.9, 125.6, 124.6, 118.1, 111.8, 67.5, 34.4. HRMS (ESI/FTICR) $m / z:[\mathrm{M}+\mathrm{H}]^{+}$calcd for $\mathrm{C}_{27} \mathrm{H}_{21} \mathrm{~N}_{4} \mathrm{O} 417.17099$; found 417.17089 .

3-(2-Methoxyphenyl)-8-phenethoxy-[1,2,4]triazolo/4,3-a]pyrazine (47f). Isolated from the same reaction as for 46f. Fractions corresponding to the first peak were combined and evaporated to give $\mathbf{4 7 f}$ as a white solid (first run: $13.1 \mathrm{mg}, 37.8 \mu \mathrm{mol}, 9 \%$, second run: 12.2 $\mathrm{mg}, 35.2 \mu \mathrm{mol}$, 9\%, average yield is $9 \%$ ). $\mathrm{mp}$ $124-128{ }^{\circ} \mathrm{C} .{ }^{1} \mathrm{H}$ NMR $\left(500 \mathrm{MHz}, \mathrm{DMSO}-d_{6}\right): \delta$ $7.71(\mathrm{~d}, J=4.8 \mathrm{~Hz}, 1 \mathrm{H}), 7.65$ (ddd, $J=8.5$, 7.4, $1.8 \mathrm{~Hz}, 1 \mathrm{H}), 7.59$ (dd, $J=7.5,1.8 \mathrm{~Hz}$, $1 \mathrm{H}), 7.42(\mathrm{~d}, J=4.9 \mathrm{~Hz}, 1 \mathrm{H}), 7.40-7.37(\mathrm{~m}$, $2 \mathrm{H}), 7.36-7.28(\mathrm{~m}, 3 \mathrm{H}), 7.27-7.20(\mathrm{~m}, 1 \mathrm{H})$, $7.18(\mathrm{td}, J=7.4,0.9 \mathrm{~Hz}, 1 \mathrm{H}), 4.76(\mathrm{t}, J=6.9$ $\mathrm{Hz}, 2 \mathrm{H}), 3.82(\mathrm{~s}, 3 \mathrm{H}), 3.19$ (t, $J=6.9 \mathrm{~Hz}, 2 \mathrm{H})$. ${ }^{13} \mathrm{C}\left\{{ }^{1} \mathrm{H}\right\} \mathrm{NMR}\left(126 \mathrm{MHz}, \mathrm{DMSO}-d_{6}\right): \delta 156.9$, $152.9,146.7,139.4,138.0,132.7,131.9,128.9$, $128.4,126.5,126.4,120.9,114.2,113.3,112.1$, 67.3, 55.6, 34.2. HRMS (ESI/FTICR) $m / z$ : $[\mathrm{M}+\mathrm{Na}]^{+}$calcd for $\mathrm{C}_{20} \mathrm{H}_{18} \mathrm{~N}_{4} \mathrm{O}_{2} \mathrm{Na} 369.13220$; found 369.13255.

\section{3-(4-Methoxyphenyl)-[1,2,4]triazolo[4,3-a]-}

pyrazin-8-ol (48a). General Procedure D was applied (with following modification: $\mathrm{H}_{2} \mathrm{O}$ was used as a solvent) using 45i (107 mg, 0.341 mmol) and 2-phenylethan-1-ol (41.7 mg, 0.341 mmol, 1.0 equiv.). The reaction mixture was purified by FCC on silica using a gradient of $\mathrm{MeOH}(0 \%$ to $20 \%)$ in EtOAc to give $48 \mathbf{a}$ as a pale brown solid (80.0 mg, $0.330 \mathrm{mmol}$, 94\%). $\quad \operatorname{mp~} 312-316{ }^{\circ} \mathrm{C}$ (decomp.). ${ }^{1} \mathrm{H}$ NMR $\left(500 \mathrm{MHz}, \mathrm{DMSO}-d_{6}\right): \delta 11.42(\mathrm{~s}, 1 \mathrm{H}), 7.81-$ $7.74(\mathrm{~m}, 2 \mathrm{H}), 7.39(\mathrm{~d}, J=5.7 \mathrm{~Hz}, 1 \mathrm{H}), 7.21-$ $7.14(\mathrm{~m}, 2 \mathrm{H}), 6.89(\mathrm{~d}, J=5.8 \mathrm{~Hz}, 1 \mathrm{H}), 3.86(\mathrm{~s}$, $3 \mathrm{H}) .{ }^{13} \mathrm{C}\left\{{ }^{1} \mathrm{H}\right\}$ NMR $\left(126 \mathrm{MHz}\right.$, DMSO- $\left.d_{6}\right): \delta$ $160.9,153.0,149.2,145.0,129.9,118.4,117.9$, 114.8, 103.8, 55.4. HRMS (ESI/FTICR) $\mathrm{m} / z$ : $[\mathrm{M}+\mathrm{Na}]^{+}$calcd for $\mathrm{C}_{12} \mathrm{H}_{10} \mathrm{~N}_{4} \mathrm{O}_{2} \mathrm{Na}$ 265.06960; found 265.06955 .

3-(4-Nitrophenyl)-[1,2,4]triazolo[4,3-a]pyrazin-8-ol (48b). General Procedure D was applied using 45j (128 mg, $0.400 \mathrm{mmol}, 1.0$ equiv.) and 2-phenylethan-1-ol (48.8 mg, 0.400 mmol, 1.0 equiv.). The reaction mixture was purified by FCC on silica using a gradient of EtOAc (30\% to 100\%) in hexanes, then $\mathrm{MeOH}$ (0\% to 20\%) in EtOAc, fractions corresponding to the third peak were combined and evaporated to give $\mathbf{4 8 b}$ as a yellow solid (first run: $28.8 \mathrm{mg}, 0.112 \mathrm{mmol}, 28 \%$, second run: 31.9 $\mathrm{mg}, 0.124 \mathrm{mmol}, 31 \%$, average yield is $30 \%)$. $\operatorname{mp} 207-210{ }^{\circ} \mathrm{C}$ (decomp.). ${ }^{1} \mathrm{H}$ NMR $(500 \mathrm{MHz}$, DMSO- $\left.d_{6}\right): \delta 8.48-8.36(\mathrm{~m}, 2 \mathrm{H}), 8.20-8.14$ $(\mathrm{m}, 2 \mathrm{H}), 7.41(\mathrm{~d}, J=4.7 \mathrm{~Hz}, 1 \mathrm{H}), 7.10(\mathrm{~d}, J=$ $4.7 \mathrm{~Hz}, 1 \mathrm{H}) .{ }^{13} \mathrm{C}\left\{{ }^{1} \mathrm{H}\right\}$ NMR $(126 \mathrm{MHz}$, DMSO$\left.d_{6}\right): \delta 159.9,147.4,146.3,144.6,133.7,132.7$, 128.3, 124.3, 99.8. HRMS (ESI/FTICR) $m / z$ : $[\mathrm{M}+\mathrm{Na}]^{+}$calcd for $\mathrm{C}_{11} \mathrm{H}_{7} \mathrm{~N}_{5} \mathrm{O}_{3} \mathrm{Na} 280.04411$; found 280.04439 .

3-(2-Methoxyphenyl)-[1,2,4]triazolo[4,3-a]pyrazin-8-ol (48c). Isolated from the same reaction as for $\mathbf{4 6 f}$. Fractions corresponding to the third peak were combined and evaporated to give $48 \mathrm{c}$ as a yellow solid (first run: 47.6 $\mathrm{mg}, 0.197 \mathrm{mmol}$, 49\%, second run: $46.3 \mathrm{mg}$, $0.191 \mathrm{mmol}, 48 \%$, average yield is $49 \%)$. mp $116-119{ }^{\circ} \mathrm{C} .{ }^{1} \mathrm{H}$ NMR $\left(500 \mathrm{MHz}, \mathrm{DMSO}-d_{6}\right): \delta$ $11.45(\mathrm{~s}, 1 \mathrm{H}), 7.65$ (ddd, $J=8.9,7.4,1.8 \mathrm{~Hz}$, $1 \mathrm{H}), 7.56(\mathrm{dd}, J=7.5,1.7 \mathrm{~Hz}, 1 \mathrm{H}), 7.29(\mathrm{dd}$, $J=8.5,0.9 \mathrm{~Hz}, 1 \mathrm{H}), 7.17(\mathrm{td}, J=7.5,1.0 \mathrm{~Hz}$, $1 \mathrm{H}), 7.00(\mathrm{~d}, J=5.7 \mathrm{~Hz}, 1 \mathrm{H}), 6.86(\mathrm{~d}, J=5.8$ $\mathrm{Hz}, 1 \mathrm{H}), 3.83(\mathrm{~s}, 3 \mathrm{H}) .{ }^{13} \mathrm{C}\left\{{ }^{1} \mathrm{H}\right\} \mathrm{NMR}(126 \mathrm{MHz}$, DMSO- $\left.d_{6}\right): \delta 156.9,152.9,147.9,144.9,132.8$, $131.9,120.9,117.7,114.2,112.1,104.8,55.7$. HRMS (ESI/FTICR) $m / z:[\mathrm{M}+\mathrm{Na}]^{+}$calcd for $\mathrm{C}_{12} \mathrm{H}_{10} \mathrm{~N}_{4} \mathrm{O}_{2} \mathrm{Na} 265.06960$; found 265.06995.

3-(2-Nitrophenyl)-[1,2,4]triazolo[4,3-a]pyrazin-8-ol (48d). Isolated from the same reaction as for $46 \mathrm{~g}$ preparation 2. Fractions corresponding to the third peak were combined and evaporated to give $\mathbf{4 8 d}$ as an orange solid (first run: $11.4 \mathrm{mg}, 44.3 \mu \mathrm{mol}, 11 \%$, second run: 13.8 
$\mathrm{mg}, 53.7 \mu \mathrm{mol}, 13 \%$, average yield is $12 \%) . \mathrm{mp}$ 124-127 ${ }^{\circ} \mathrm{C}$ (decomp.). ${ }^{1} \mathrm{H}$ NMR (500 MHz, DMSO- $\left.d_{6}\right): \delta 11.65(\mathrm{~s}, 1 \mathrm{H}), 8.37(\mathrm{dd}, J=8.1$, $1.3 \mathrm{~Hz}, 1 \mathrm{H}), 8.02(\mathrm{td}, J=7.5,1.4 \mathrm{~Hz}, 1 \mathrm{H})$, $7.97(\mathrm{td}, J=7.8,1.6 \mathrm{~Hz}, 1 \mathrm{H}), 7.87(\mathrm{dd}, J=$ 7.5, $1.6 \mathrm{~Hz}, 1 \mathrm{H}), 7.20(\mathrm{~d}, J=5.6 \mathrm{~Hz}, 1 \mathrm{H})$, $6.93(\mathrm{~d}, J=5.7 \mathrm{~Hz}, 1 \mathrm{H}) .{ }^{13} \mathrm{C}\left\{{ }^{1} \mathrm{H}\right\} \mathrm{NMR}(126$ $\left.\mathrm{MHz}, \mathrm{DMSO}-d_{6}\right): \delta 152.7,148.1,146.2,144.9$, 134.6, 133.1, 132.7, 125.4, 119.9, 118.9, 103.7. HRMS (ESI/FTICR) $m / z:[\mathrm{M}+\mathrm{H}]^{+}$calcd for $\mathrm{C}_{11} \mathrm{H}_{7} \mathrm{~N}_{5} \mathrm{O}_{3} \mathrm{Na} 280.04411$; found 280.04426.

3-(4-Methoxyphenyl)-[1,2,4]-triazolo[4,3alpyrazine (49). Preparation 1: General Procedure E was applied using 45n (341 mg, 0.968 mmol, 1.0 equiv.) and 2-phenylethan-1-amine (352 mg, $2.91 \mathrm{mmol}, 3.0$ equiv.). Fractions corresponding to the second peak were re-purified by RP-FCC on $\mathrm{C} 18$ using a gradient of $\mathrm{MeOH}$ ( $5 \%$ to $80 \%$ ) in $\mathrm{H}_{2} \mathrm{O}$. Fractions corresponding to the first peak were combined and evaporated to give 49 as a white solid $(24.0 \mathrm{mg}, 0.106 \mathrm{mmol}$, 11\%). Preparation 2: General Procedure D was applied using 45n (108 mg, $0.307 \mathrm{mmol}, 1.0$ equiv.) and 2-phenylethane-1-thiol $(43.0 \mathrm{mg}$, $0.307 \mathrm{mmol}, 1.0$ equiv.). The reaction mixture was purified by FCC on silica using a gradient of $\mathrm{MeOH}$ (0\% to 10\%) in DCM, fractions corresponding to the second peak were evaporated to give 49 as a yellow solid $(51.0 \mathrm{mg}$, $0.225 \mathrm{mmol}, 74 \%) . \operatorname{mp} 202-205{ }^{\circ} \mathrm{C} .{ }^{1} \mathrm{H}$ NMR $\left(500 \mathrm{MHz}\right.$, DMSO- $\left.d_{6}\right): \delta 9.45(\mathrm{~d}, J=1.6 \mathrm{~Hz}$, $1 \mathrm{H}), 8.57(\mathrm{dd}, J=4.9,1.6 \mathrm{~Hz}, 1 \mathrm{H}), 7.94(\mathrm{~d}$, $J=4.9 \mathrm{~Hz}, 1 \mathrm{H}), 7.93-7.87(\mathrm{~m}, 2 \mathrm{H}), 7.23$ $-7.16(\mathrm{~m}, 2 \mathrm{H}), 3.87(\mathrm{~s}, 3 \mathrm{H}) .{ }^{13} \mathrm{C}\left\{{ }^{1} \mathrm{H}\right\} \mathrm{NMR}$ $\left(126 \mathrm{MHz}, \mathrm{DMSO}-d_{6}\right): \delta 160.9,146.5,145.5$, 144.1, 129.8, 129.7, 117.8, 116.9, 114.8, 55.4. HRMS (ESI/FTICR) $m / z:[\mathrm{M}+\mathrm{Na}]^{+}$calcd for $\mathrm{C}_{12} \mathrm{H}_{10} \mathrm{~N}_{4} \mathrm{ONa} 249.07468$; found 249.07465.

3-(4-Methoxyphenyl)-5-(1-phenethyl-1H-imidazol-2-yl)-4H-1,2,4-triazole (50). Isolated from the same reaction as for $\mathbf{4 9}$ preparation 1. Fractions corresponding to the second peak, after RP-FCC were combined and evaporated to give 50 as a white solid $(57.0 \mathrm{mg}, 0.165$ mmol, 17\%). mp $143-146{ }^{\circ} \mathrm{C} .{ }^{1} \mathrm{H}$ NMR (500 $\left.\mathrm{MHz}, \mathrm{CD}_{3} \mathrm{OD}\right): \delta 8.00(\mathrm{~d}, J=8.8 \mathrm{~Hz}, 2 \mathrm{H})$, $7.29-7.17(\mathrm{~m}, 2 \mathrm{H}), 7.19-7.12(\mathrm{~m}, 4 \mathrm{H}), 7.07$ $(\mathrm{d}, J=8.8 \mathrm{~Hz}, 2 \mathrm{H}), 7.05(\mathrm{~s}, 1 \mathrm{H}), 4.78(\mathrm{t}, J$ $=7.4 \mathrm{~Hz}, 2 \mathrm{H}), 3.87(\mathrm{~s}, 3 \mathrm{H}), 3.12(\mathrm{t}, J=7.3$
$\mathrm{Hz}, 2 \mathrm{H}) \cdot{ }^{13} \mathrm{C}\left\{{ }^{1} \mathrm{H}\right\} \mathrm{NMR}\left(126 \mathrm{MHz}, \mathrm{CD}_{3} \mathrm{OD}\right): \delta$ $162.9,139.3,129.9,129.5,129.1,127.7,124.0$, 115.4, 55.9, 50.0, 38.6. HRMS (ESI/FTICR) $m / z:[\mathrm{M}+\mathrm{H}]^{+}$calcd for $\mathrm{C}_{20} \mathrm{H}_{20} \mathrm{~N}_{5} \mathrm{O} 346.16624$; found 346.16560. X-ray single crystal data can be found in the supporting information.

5-(Piperidin-1-yl)-[1,2,4]triazolo[4,3-a]pyrazine (55). General Procedure E was applied using 45a (101 mg, $0.652 \mathrm{mmol}, 1.0$ equiv.) in toluene $(10 \mathrm{~mL})$ and piperidine $(167 \mathrm{mg}$, $1.96 \mathrm{mmol}, 3.0$ equiv.) and heated at reflux for $72 \mathrm{~h}$. The reaction mixture was purified by FCC on silica using a gradient of EtOAc $(50 \%$ to $100 \%)$ in hexanes, then $\mathrm{MeOH}(0 \%$ to $5 \%)$ in EtOAc. Fractions corresponding to the second peak were evaporated to give 55 as an orange crystalline solid (20.7 mg, $0.102 \mathrm{mmol}$, 16\%). $\operatorname{mp~} 158-161{ }^{\circ} \mathrm{C} .{ }^{1} \mathrm{H}$ NMR $(500 \mathrm{MHz}$, DMSO- $\left.d_{6}\right): \delta 9.39(\mathrm{~d}, J=0.8 \mathrm{~Hz}, 1 \mathrm{H}), 9.05$ $(\mathrm{d}, J=0.7 \mathrm{~Hz}, 1 \mathrm{H}), 7.48(\mathrm{~s}, 1 \mathrm{H}), 3.23-3.05$ $(\mathrm{m}, 4 \mathrm{H}), 1.76(\mathrm{p}, J=5.8 \mathrm{~Hz}, 4 \mathrm{H}), 1.67-1.58$ $(\mathrm{m}, 2 \mathrm{H}) .{ }^{13} \mathrm{C}\left\{{ }^{1} \mathrm{H}\right\}$ NMR $\left(126 \mathrm{MHz}\right.$, DMSO- $\left.d_{6}\right)$ : $\delta$ 145.6, 138.4, 135.7, 134.5, 116.4, 50.2, 25.0, 23.6. HRMS (ESI/FTICR) $m / z:[\mathrm{M}+\mathrm{H}]^{+}$ calcd for $\mathrm{C}_{10} \mathrm{H}_{14} \mathrm{~N}_{5}$ 204.12437; found 204.12426.

8-(Piperidin-1-yl)-[1,2,4]triazolo[4,3-a]pyrazine (56). Isolated from the same reaction as for 55. Fractions corresponding to the first peak were evaporated to give $\mathbf{5 6}$ as an orange crystalline solid (93.4 mg, $0.460 \mathrm{mmol}, 71 \%$ ). mp $181-183{ }^{\circ} \mathrm{C} .{ }^{1} \mathrm{H}$ NMR $\left(500 \mathrm{MHz}, \mathrm{CD}_{3} \mathrm{CN}\right)$ : $\delta 8.82(\mathrm{~s}, 1 \mathrm{H}), 7.56(\mathrm{~d}, J=4.5 \mathrm{~Hz}, 1 \mathrm{H}), 7.26$ $(\mathrm{d}, J=4.6 \mathrm{~Hz}, 1 \mathrm{H}), 4.25(\mathrm{t}, J=5.4 \mathrm{~Hz}, 4 \mathrm{H})$, $1.78-1.70(\mathrm{~m}, 2 \mathrm{H}), 1.66(\mathrm{dd}, J=7.6,3.9$ $\mathrm{Hz}, 4 \mathrm{H}) .{ }^{13} \mathrm{C}\left\{{ }^{1} \mathrm{H}\right\}$ NMR $\left(126 \mathrm{MHz}, \mathrm{CD}_{3} \mathrm{CN}\right)$ : $\delta$ 148.8, 141.5, 138.3, 129.9, 108.3, 48.1, 26.9, 25.5. HRMS (ESI/FTICR) $m / z:[\mathrm{M}+\mathrm{H}]^{+}$ calcd for $\mathrm{C}_{10} \mathrm{H}_{14} \mathrm{~N}_{5} 204.12437$; found 204.12412. $\mathrm{X}$-ray single crystal data can be found in the supporting information.

5-(Piperidin-1-yl)-[1,2,4]triazolo[4,3-a]pyrazine-3-d (57). General Procedure E was applied using 45a (101 mg, 0.652 mmol, 1.0 equiv.) and piperidine (167 $\mathrm{mg}, 1.96 \mathrm{mmol}$, 3.0 equiv.) in $\mathrm{D}_{2} \mathrm{O}(5 \mathrm{~mL})$. The reaction mixture was heated at reflux for $72 \mathrm{~h}$ and purified by FCC on silica using a gradient of EtOAc (50\% to $100 \%)$ in hexanes then $\mathrm{MeOH}(0 \%$ to $5 \%$ in EtOAc. Fractions corresponding to the 
second peak were evaporated to give 57 as an orange crystalline solid (31.1 mg, $0.153 \mathrm{mmol}$, $23 \%$ ). mp $158-161{ }^{\circ} \mathrm{C} .{ }^{1} \mathrm{H}$ NMR $(500 \mathrm{MHz}$, DMSO- $\left.d_{6}\right): \delta 9.05(\mathrm{~s}, 1 \mathrm{H}), 7.48(\mathrm{~s}, 1 \mathrm{H}), 3.19-$ $3.13(\mathrm{~m}, 4 \mathrm{H}), 1.75(\mathrm{p}, J=5.7 \mathrm{~Hz}, 4 \mathrm{H}), 1.66-$ $1.58(\mathrm{~m}, 2 \mathrm{H}) .{ }^{2} \mathrm{H}$ NMR $\left(77 \mathrm{MHz}, \mathrm{DMSO}-d_{6}\right): \delta$ 9.44 (s, 1D). ${ }^{13} \mathrm{C}\left\{{ }^{1} \mathrm{H}\right\}$ NMR (126 MHz, DMSO$\left.d_{6}\right): \delta$ 145.6, 138.3, 135.7, $134.7-133.9(\mathrm{~m})$, 116.3, 50.2, 25.0, 23.6. HRMS (ESI/FTICR) $m / z:[\mathrm{M}+\mathrm{H}]^{+}$calcd for $\mathrm{C}_{10} \mathrm{H}_{13} \mathrm{DN}_{5}$ 205.13065; found 205.13038.

\section{8-(Piperidin-1-yl)-[1,2,4]triazolo[4,3-a]pyra-} zine-3,5- $d_{2}$ (58). Isolated from the same reaction as for 57. Fractions corresponding to the first peak were evaporated to give $\mathbf{5 8}$ as an orange crystalline solid (78.7 mg, 0.387 mmol, 59\%). $\operatorname{mp~} 181-183{ }^{\circ} \mathrm{C} .{ }^{1} \mathrm{H}$ NMR (500 $\left.\mathrm{MHz}, \mathrm{DMSO}-d_{6}\right): \delta 7.31(\mathrm{~s}, 1 \mathrm{H}), 4.21(\mathrm{t}, J=$ $5.5 \mathrm{~Hz}, 4 \mathrm{H}$ ), 1.68 (tt, $J=6.4,2.4 \mathrm{~Hz}, 2 \mathrm{H}$ ), 1.61 (tq, $J=8.4,5.3,4.2 \mathrm{~Hz}, 4 \mathrm{H}) .{ }^{2} \mathrm{H}$ NMR $\left(77 \mathrm{MHz}\right.$, DMSO- $\left.d_{6}\right): \quad \delta 9.28$ (s, 1D), 7.88 (s, 1D). ${ }^{13} \mathrm{C}\left\{{ }^{1} \mathrm{H}\right\}$ NMR $\left(126 \mathrm{MHz}\right.$, DMSO- $d_{6}$ $\delta$ 147.1, 139.6, 137.86 - 137.14 (m), 128.6, $107.41(\mathrm{t}, J=29.3 \mathrm{~Hz}), 46.6,25.7,24.2$. HRMS (ESI/FTICR) $m / z:[\mathrm{M}+\mathrm{H}]^{+}$calcd for $\mathrm{C}_{10} \mathrm{H}_{12} \mathrm{D}_{2} \mathrm{~N}_{5}$ 206.13693; found 206.13662.

5-Chloro-[1,2,4]triazolo[4,3-a]pyrazine-3-d

(59). Compound 45a (227 mg, $1.47 \mathrm{mmol}$ ) was stirred in $\mathrm{D}_{2} \mathrm{O}(5 \mathrm{~mL})$ at $80{ }^{\circ} \mathrm{C}$ for 2 days. The solvent was evaporated and the reaction mixture was purified by $\mathrm{FCC}$ on silica using a gradient of EtOAc (20 to 100\%) in hexanes to give $\mathbf{5 9}$ as a white solid (197 mg, 1.27 mmol, 86\%). mp $170-173{ }^{\circ} \mathrm{C} .{ }^{1} \mathrm{H}$ NMR (500 $\left.\mathrm{MHz}, \mathrm{CDCl}_{3}\right): \delta 9.30(\mathrm{~s}, 1 \mathrm{H}), 7.95(\mathrm{~s}, 1 \mathrm{H})$. ${ }^{2} \mathrm{H} \mathrm{NMR}\left(77 \mathrm{MHz}, \mathrm{CDCl}_{3}\right): \delta 9.10(\mathrm{~s}, 1 \mathrm{D})$. ${ }^{13} \mathrm{C}\left\{{ }^{1} \mathrm{H}\right\}$ NMR $\left(126 \mathrm{MHz}, \mathrm{CDCl}_{3}\right): \delta$ 145.9, 142.0, 134.96 - $134.04(\mathrm{~m}), 128.4,121.3$. LRMS $(\mathrm{ESI} / \mathrm{IT}) m / z:[\mathrm{M}+\mathrm{H}]^{+}$156.0.

8-(Piperidin-1-yl)-[1,2,4]triazolo[4,3-a]pyrazine-3-d (60). 56 (10 mg, $49 \mu \mathrm{mol})$ was dissolved in $\mathrm{D}_{2} \mathrm{O}(5 \mathrm{~mL})$ and heated at reflux for $72 \mathrm{~h}$. Solvent was evaporated to give $\mathbf{6 0}$ as an orange solid (10 mg, $49 \mu \mathrm{mol}, 100 \%)$. mp 181$183{ }^{\circ} \mathrm{C} .{ }^{1} \mathrm{H} \mathrm{NMR}\left(500 \mathrm{MHz}, \mathrm{CDCl}_{3}\right): \delta 7.37(\mathrm{~d}$, $J=4.5 \mathrm{~Hz}, 1 \mathrm{H}), 7.31(\mathrm{~d}, J=4.5 \mathrm{~Hz}, 1 \mathrm{H}), 4.30$ (s, 4H), $1.72(\mathrm{~d}, J=7.5 \mathrm{~Hz}, 6 \mathrm{H}) .{ }^{2} \mathrm{H} \operatorname{NMR}(77$ $\left.\mathrm{MHz}, \mathrm{CDCl}_{3}\right): \delta 8.75(\mathrm{~s}, 1 \mathrm{D}) .{ }^{13} \mathrm{C}\left\{{ }^{1} \mathrm{H}\right\} \mathrm{NMR}$ $\left(126 \mathrm{MHz}, \mathrm{CDCl}_{3}\right): \delta 148.1,140.7,137.1-$ $136.1(\mathrm{~m}), 130.0,106.0,47.6,26.4,24.9$. LRMS $(\mathrm{ESI} / \mathrm{IT}) m / z:[\mathrm{M}+\mathrm{Na}]^{+} 227.1$.

Acknowledgement We thank Mark Coster for suggestions on the mechanism of the telesubstitution reaction; Chris Swain, Chase Smith and other members of the OSM consortium for their valuable discussion on GitHub; Peter Turner for the help with X-ray crystallography; Irene Hallyburton for conducting in vitro antiplasmodial activity assay and the Structural Genomics Consortium at The University of North Carolina at Chapel Hill (SGC-UNC) for facilitating the KINOMEscan ${ }^{\circledR}$ assay. Also we thank the Australian Research Council and the Medicines for Malaria Venture for support (LP150101226).

\section{Supporting Information Avail- able}

The Supporting Information is available free of charge on the ACS Publications website at DOI: The following files are available free of charge.

- ORTEP diagrams for the X-ray structures and crystal data; experimental details for biological activity evaluations and copies of ${ }^{1} \mathrm{H}$ and ${ }^{13} \mathrm{C}\left\{{ }^{1} \mathrm{H}\right\}$ NMR spectra of novel compounds. (PDF)

- The archive of laboratory notebook with all experiments described in the article and raw NMR data for all novel compounds. (ZIP)

- The KINOMEscan ${ }^{\circledR}$ assay report on the biological activity of compounds $\mathbf{4 6 d}$ and 47b. (XLS)

- X-ray crystal data of $45 a, 50,56$. (CIF)

- The structural information in strings formant for all compounds described in the article with reference codes to the laboratory notebook. (XLS) 


\section{References}

(1) IUPAC Gold Book (accessed Jan 15, 2020). https://goldbook.iupac.org/ terms/view/I03251.

(2) IUPAC Gold Book (accessed Jan 15, 2020). https://goldbook.iupac.org/ $\mathrm{html} / \mathrm{C} / \mathrm{C} 01081$.html.

(3) IUPAC Gold Book (accessed Jan 15, 2020). https://goldbook.iupac.org/ html/T/T06256.html.

(4) Open Source Malaria Project. Offcial website (accessed Jan 15, 2020). http:// opensourcemalaria.org/.

(5) Runde, M. M.; Scott, E. W.; Johnson, J. R. Rearrangement of the AlphaFurfuryl Group. 2-Furylacetic Acid and 5Methylfuroic Acid. Journal of the American Chemical Society 1930, 52, 12841289 .

(6) Torr, J. E.; Large, J. M.; Horton, P. N.; Hursthouse, M. B.; McDonald, E. On the nucleophilic telesubstitution of dichloropyrazines by metallated dithianes. Tetrahedron Letters 2006, 47, 31-34.

(7) Verček, B.; Stanovnik, B.; Tišler, M. Products of abnormal substitution of striazolo(1,5-a)pyrazines. Tetrahedron Letters 1974, 15, 4539-4542.

(8) Bradac, J.; Furek, Z.; Janezic, D.; Molan, S.; Smerkolj, I.; Stanovnik, B.; Tisler, M.; Vercek, B. Heterocycles. 167. Telesubstitution and other transformations of imidazo[1,2-a]- and s-triazolo[4,3a]pyrazines. The Journal of Organic Chemistry 1977, 42, 4197-4201.

(9) Suwiński, J. W. cine- and teleSubstitution reactions: review of work from 2002-2016. Arkivoc 2017, 2017, 402-435.

(10) Suwiński, J.; Świerczek, K. cine - and tele Substitution reactions. Tetrahedron 2001, 57, 1639-1662.
(11) Tišler, M. Tele-substitutions in Heterocyclic Chemistry. Acta chimica Slovenica 2011, 58, 9-13.

(12) Terrier, F. Modern Nucleophilic Aromatic Substitution; Wiley-VCH, 2013.

(13) Open Source Malaria Project wiki (accessed Jan 15, 2020). https://github. com/OpenSourceMalaria/Series4/wiki.

(14) Open Source Malaria Project wiki (accessed Jan 15, 2020). https://github.com/ OpenSourceMalaria/Series4/wiki/ Modification-of-the-Triazole-Substitution.

(15) Open Source Malaria Project wiki (accessed Jan 15, 2020). https: //github.com/OpenSourceMalaria/ Series4/wiki/In-Vivo-Efficacy.

(16) Lee, S.; Cil, O.; Diez-Cecilia, E.; Anderson, M. O.; Verkman, A. S. NanomolarPotency 1,2,4-Triazoloquinoxaline Inhibitors of the Kidney Urea Transporter UT-A1. Journal of Medicinal Chemistry 2018, 61, 3209-3217.

(17) Pasternak, A.; Pio, B.; Chobanian, R., Harry; Shi, Z.-C.; Dong, S.; Guo, Y.; Walsh, P., Shawn; Guo, Z.; Ferguson, D., Ronald; Cato, B. Inhibitors of the renal outer medullary potassium channel. WO2016/060941. April 21, 2016.

(18) Aschner, P.; Kipnes, M. S.; Lunceford, J. K.; Sanchez, M.; Mickel, C.; and, D. E. W.-H. Effect of the Dipeptidyl Peptidase-4 Inhibitor Sitagliptin as Monotherapy on Glycemic Control in Patients With Type 2 Diabetes. Diabetes Care 2006, 29, 2632-2637.

(19) Ali, I.; Lee, J.; Go, A.; Choi, G.; Lee, K. Discovery of novel $[1,2,4]$ triazolo[4,3- a ]quinoxaline aminophenyl derivatives as BET inhibitors for cancer treatment. Bioorganic \& Medicinal Chemistry Letters 2017, 27, 4606-4613. 
(20) Shapiro, G. 3,3-Difluoropiperidine carbamate heterocyclic comopunds as NR2B NMDA receptor antagonists. WO2016/196513. December 8, 2016.

(21) Github. Open Source Malaria Project (accessed Jan 15, 2020). https://github. com/OpenSourceMalaria/Series4/ issues/39\#issuecomment-391222820.

(22) Mal, S.; Prathap, K. J.; Smith, S. C.; Umarye, J. D. Facile one pot synthesis of 8-chloro- $[1,2,4]$ triazolo[4,3-a]pyrazines via oxidative cyclisation using chloramine $\mathrm{T}$. Tetrahedron Letters 2015, 56, 2896-2901.

(23) Helal, C. J. et al. Application of StructureBased Design and Parallel Chemistry to Identify a Potent, Selective, and Brain Penetrant Phosphodiesterase 2A Inhibitor. Journal of Medicinal Chemistry 2017, 60, 5673-5698.

(24) Viswanathan, B.; Jacob, B. Alkylation, Hydrogenation and Oxidation Catalyzed by Mesoporous Materials. Catalysis Reviews 2005, 47, 1-82.

(25) Venuto, P.; Hamilton, L.; Landis, P.; Wise, J. Organic reactions catalyzed by crystalline aluminosilicatesI. Alkylation reactions. Journal of Catalysis 1966, 5, $81-98$.

(26) Zaydoun, S.; Idrissi, M. S.; GarrigouLagrange, C. Étude vibrationnelle du méthyl-4 triazole-1,2,4 et de son chlorhydrate. Canadian Journal of Chemistry 1987, 65, 2509-2512.

(27) Hurd, J. A.; Vaidhyanathan, R.; Thangadurai, V.; Ratcliffe, C. I.; Moudrakovski, I. L.; Shimizu, G. K. H. Anhydrous proton conduction at $150{ }^{\circ} \mathrm{C}$ in a crystalline metal-organic framework. Nature Chemistry 2009, 1, 705-710.

(28) Wu, P.; Nielsen, T. E.; Clausen, M. H. FDA-approved small-molecule kinase inhibitors. Trends in Pharmacological Sciences 2015, 36, 422-439.
(29) Liu, W.; Li, J.; He, K.; Huang, F.; Ma, Y.; Li, Y.; Li, Q.; Xu, F. Synthesis, bioactivity, action mode and 3D-QSAR of novel anthranilic diamide derivatives. Chinese Chemical Letters 2019, 30, 417-420.

(30) Tam, H. D.; Sarfati, R. S.; Gouyette, C.; Igolen, J.; Bisagni, E.; Lhoste, J. M.; Civier, A. Synthesis of C-nucleosides. 17. s-Triazolo[4,3-a]pyrazines. The Journal of Organic Chemistry 1979, 44, 1028-1035.

(31) Calderwood, D. J.; Bonafoux, D. F.; Burchat, A.; Ding, P.; Frank, K. E.; Hoemann, M. Z.; Mullen, K. D.; Davis, H. M. Novel Triazolopyridazines. WO/2009/005675. January 8, 2009.

(32) Kanaya, N.; Ishiyama, T.; Muto, R.; Ochiai, Y.; Watanabe, T.; Shima, N. Amidopyrazole Derivative. EP1698626 (A1). September 06, 2006.

(33) Kim, D. et al. (2R)-4-Oxо4-[3-(Trifluoromethyl)-5,6-

dihydro[1,2,4]triazolo[4,3-a]pyrazin$7(8 \mathrm{H})$-yl]-1-(2,4,5-trifluorophenyl)butan2-amine: A Potent, Orally Active Dipeptidyl Peptidase IV Inhibitor for the Treatment of Type 2 Diabetes. Journal of Medicinal Chemistry 2005, 48, 141-151.

(34) Rasmussen, L.; Demmer, C.; Jørgensen, M.; Kehler, J.; Bunch, L. Synthesis and Selective Functionalization of $[1,2,4]$ Triazolo-[4,3-a]pyrazines. Synlett 2015, 26, 519-524.

(35) Jackson, J. J.; Kobayashi, H.; Steffens, S. D.; Zakarian, A. 10-Step Asymmetric Total Synthesis and Stereochemical Elucidation of ()-Dragmacidin D. Angewandte Chemie International Edition 2015, 54, 9971-9975. 


\section{Graphical TOC Entry}

$\begin{array}{llllll}\text { Unusual } & & & \text { ipso } & \text { tele } \\ \text { tele-substitution } & \mathrm{NuH} & =\mathrm{ROH} & \mathrm{RSH} & \mathrm{RNH}_{2} \\ \text { in bioactive } & \mathrm{X} & =\mathrm{Cl} & \mathrm{Br} & \mathrm{I} \\ \text { compounds } & \mathrm{Ar} & =\mathrm{EWG} & \mathrm{EDG} & \end{array}$




\section{Supporting information Tele-substitution Reactions in the Synthesis of a Promising Class of Antimalarials}

Marat Korsik, Edwin G. Tse, ${ }^{\dagger, \ddagger}$ David G. Smith, ${ }^{\uparrow}$ William Lewis, ${ }^{\dagger}$ Peter J. Rutledge, ${ }^{*, \dagger}$ and Matthew H. Todd ${ }^{*, \dagger}$

$\dagger$ School of Chemistry, The University of Sydney, Sydney, NSW 2006, Australia

$\ddagger$ School of Pharmacy, University College London, 29-39 Brunswick Square, London WC1N $1 A X, U . K$.

ISchool of Health and Life Sciences, Federation University, Gippsland Campus, VIC 3842, Australia

E-mail: peter.rutledge@sydney.edu.au; matthew.todd@ucl.ac.uk Phone: +6129351 5020; +442077535568 


\section{Contents}

S1 Biological activity experimental details $\quad$ S3

S1.1 In Vitro Antiplasmodial Activity . . . . . . . . . . . . . . . . . . . . S3

S1.2 KINOMEscan ${ }^{\circledR}$ Assay . . . . . . . . . . . . . . . . . . . . S3

S2 General synthetic route to make studied triazolopyrazines $\quad$ S4

S3 Mechanism of transformation that leads to the ring opening product $50 \quad$ S4

$\begin{array}{lr}\text { S4 NMR spectra of compounds } & \text { S4 }\end{array}$

2-Bromo-6-hydrazinylpyrazine $\mathbf{S 2} \ldots \ldots \ldots \ldots$. . . . . . . . . . . . . . . . . . . . . . . . .

2-Iodo-6-hydrazinylpyrazine $\mathbf{S 3} \ldots \ldots \ldots \ldots$. . . . . . . . . . . . S6

2-Chloro-3-hydrazinylpyrazine $\mathbf{S 4} \ldots \ldots \ldots \ldots$. . . . . . . . . . . . . . . . . . . . . . . . . . .

2-Chloro-5-hydrazinylpyrazine $\mathbf{S 5} \ldots \ldots \ldots \ldots$. . . . . . . . . . . S7

5-Chloro-3-(4-(difluoromethoxy)phenyl)-[1,2,4]triazolo[4,3-a]pyrazine $16 \ldots \ldots$. . . . . . . S9

3-(4-(Difluoromethoxy)phenyl)-5-(phenethylthio)-[1,2,4]triazolo[4,3-a]pyrazine 17 . . . . . S10

3-(4-(Difluoromethoxy)phenyl)-8-(phenethylthio)-[1,2,4]triazolo[4,3-a]pyrazine 18 . . . . . S11

8-Chloro- $[1,2,4]$ triazolo $[4,3-a]$ pyrazine $25 \ldots \ldots \ldots$. . . . . . . . . . . . S12

8-Chloro-3-phenyl-[1,2,4]triazolo[4,3-a]pyrazine $26 \ldots \ldots \ldots$. . . . . . . . . . . . S13

8-Chloro-3-(4-nitrophenyl)-[1,2,4]triazolo[4,3-a]pyrazine 27 . . . . . . . . . . . . . . S14

8-Phenethoxy-[1,2,4]triazolo[4,3-a]pyrazine $28 \ldots \ldots \ldots \ldots$. . . . . . . . . S15

8-(Phenethylthio)-[1,2,4]triazolo[4,3-a]pyrazine $29 \ldots \ldots \ldots \ldots$. . . . . . . . S16

$N$-Phenethyl-[1,2,4]triazolo[4,3-a]pyrazin-8-amine $30 \ldots \ldots \ldots$. . . . . . . . . S17

8-Phenethoxy-3-phenyl-[1,2,4]triazolo[4,3-a]pyrazine $31 \ldots \ldots \ldots$. . . . . . . . S18

8-(Phenethylthio)-3-phenyl-[1,2,4]triazolo[4,3-a]pyrazine 32 . . . . . . . . . . . . . . . S19

$N$-Phenethyl-3-phenyl-[1,2,4]triazolo[4,3-a]pyrazin-8-amine 33 . . . . . . . . . . . . . . S20

3-(4-Nitrophenyl)-8-phenethoxy-[1,2,4]triazolo[4,3-a]pyrazine $34 \ldots \ldots$. . . . . . . . S21

3-(4-Nitrophenyl)-8-(phenethylthio)-[1,2,4]triazolo[4,3-a]pyrazine 35 . . . . . . . . . . . . S22

3-(4-Nitrophenyl)- $N$-phenethyl-[1,2,4] triazolo[4,3-a]pyrazin-8-amine 36 . . . . . . . . . S23

6-Chloro- $[1,2,4]$ triazolo $[4,3-a]$ pyrazine 37 . . . . . . . . . . . . . . . . . . S24

6-Chloro-3-(4-(difluoromethoxy)phenyl)-[1,2,4]triazolo[4,3-a]pyrazine 38 . . . . . . . . S25

2-Chloro-6-phenethoxypyrazine 42 . . . . . . . . . . . . . . . . S26

2-Bromo-6-phenethoxypyrazine 43 . . . . . . . . . . . . . . . . . . S27

2-Iodo-6-phenethoxypyrazine $44 \ldots \ldots \ldots \ldots$. . . . . . . . . . S28

5-Chloro- $[1,2,4]$ triazolo $[4,3-a]$ pyrazine $45 \mathrm{a} \ldots \ldots \ldots \ldots$. . . . . . . . . . . . . . S29

5-Chloro-3-(4-methoxyphenyl)-[1,2,4]triazolo[4,3-a]pyrazine 45b . . . . . . . . . . . . . S31

5-Chloro-3-(4-nitrophenyl)-[1,2,4]triazolo[4,3-a]pyrazine 45c . . . . . . . . . . . . . . . . S32

5-Chloro-3-(2-methoxyphenyl)-[1,2,4]triazolo[4,3-a]pyrazine 45d . . . . . . . . . . . . S33

5-Chloro-3-(2-nitrophenyl)-[1,2,4]triazolo[4,3-a]pyrazine 45e . . . . . . . . . . . . . S34

5-Chloro-3-(3,5-di-tert-butylphenyl)-[1,2,4]triazolo[4,3-a]pyrazine 45f . . . . . . . . . . S35

3-(Anthracen-9-yl)-5-chloro-[1,2,4]triazolo[4,3-a]pyrazine 45g . . . . . . . . . . . . S36

5-Bromo- $[1,2,4]$ triazolo[4,3-a]pyrazine 45h . . . . . . . . . . . . . . . . . . S37

5-Bromo-3-(4-methoxyphenyl)-[1,2,4]triazolo[4,3-a]pyrazine 45i . . . . . . . . . . . . . . S38

5-Bromo-3-(4-nitrophenyl)-[1,2,4]triazolo[4,3-a]pyrazine 45j . . . . . . . . . . . . . . . . . S39

5-Bromo-3-(2-methoxyphenyl)-[1,2,4] triazolo[4,3-a]pyrazine 45k . . . . . . . . . . . . . . S40

5-Bromo-3-(2-nitrophenyl)-[1,2,4]triazolo[4,3-a]pyrazine 45l . . . . . . . . . . . . . . . . S41

5-Iodo-[1,2,4]triazolo[4,3-a]pyrazine 45m . . . . . . . . . . . . . . . . . . . . S42

5-Iodo-3-(4-methoxyphenyl)-[1,2,4]triazolo[4,3-a]pyrazine 45n . . . . . . . . . . . . . . . S43 
5-Phenethoxy-[1,2,4]triazolo[4,3-a]pyrazine 46a . . . . . . . . . . . . . . . . . . S44

5-(Phenethylthio)-[1,2,4]triazolo[4,3-a]pyrazine 46b . . . . . . . . . . . . . . S45

3-(4-Methoxyphenyl)-5-phenethoxy-[1,2,4]triazolo[4,3-a]pyrazine 46c . . . . . . . . . . . . S46

3-(4-Methoxyphenyl)-5-(phenethylthio)-[1,2,4]triazolo[4,3-a]pyrazine 46d . . . . . . . . S47

3-(4-Nitrophenyl)-5-phenethoxy-[1,2,4]triazolo[4,3-a]pyrazine 46e . . . . . . . . . . . . . S48

3-(2-Methoxyphenyl)-5-phenethoxy-[1,2,4]triazolo[4,3-a]pyrazine 46f . . . . . . . . . . . . S49

3-(2-Nitrophenyl)-5-phenethoxy-[1,2,4]triazolo[4,3-a]pyrazine 46g . . . . . . . . . . . . . S50

3-(3,5-Di-tert-butylphenyl)-5-phenethoxy-[1,2,4]triazolo[4,3-a]pyrazine $46 \mathbf{6}$. . . . . . . . S51

3-(Anthracen-9-yl)-5-phenethoxy-[1,2,4]triazolo[4,3-a]pyrazine 46i . . . . . . . . . . . . S52

3-(4-Methoxyphenyl)-8-phenethoxy-[1,2,4]triazolo[4,3-a]pyrazine 47a . . . . . . . . . . . S53

3-(4-Methoxyphenyl)-8-(phenethylthio)-[1,2,4]triazolo[4,3-a]pyrazine 47b . . . . . . . . . S54

3-(4-Methoxyphenyl)- $N$-phenethyl-[1,2,4]triazolo[4,3-a]pyrazin-8-amine 47c . . . . . . . . S55

3-(3,5-Di-tert-butylphenyl)-8-phenethoxy-[1,2,4]triazolo[4,3-a]pyrazine 47d . . . . . . . S56

3-(Anthracen-9-yl)-8-phenethoxy-[1,2,4] triazolo[4,3-a]pyrazine 47e . . . . . . . . . . . . . S57

3-(2-Methoxyphenyl)-8-phenethoxy-[1,2,4]triazolo[4,3-a]pyrazine 47f . . . . . . . . . . . . S58

3-(4-Methoxyphenyl)-[1,2,4]triazolo[4,3-a]pyrazin-8-ol 48a . . . . . . . . . . . . . . . S59

3-(4-Nitrophenyl)-[1,2,4]triazolo[4,3-a]pyrazin-8-ol 48b . . . . . . . . . . . . . . . . S60

3-(2-Methoxyphenyl)-[1,2,4] triazolo[4,3-a]pyrazin-8-ol 48c . . . . . . . . . . . . . . . . S61

3-(2-Nitrophenyl)-[1,2,4]triazolo[4,3-a]pyrazin-8-ol 48d . . . . . . . . . . . . . . . . S62

3-(4-Methoxyphenyl)-[1,2,4] triazolo[4,3-a]pyrazine 49 . . . . . . . . . . . . . . . . . . . . S63

3-(4-Methoxyphenyl)-5-(1-phenethyl-1H-imidazol-2-yl)-4H-1,2,4-triazole 50 . . . . . . . . S64

5 -(Piperidin-1-yl)-[1,2,4]triazolo[4,3-a]pyrazine $55 \ldots \ldots \ldots$. . . . . . . . S65

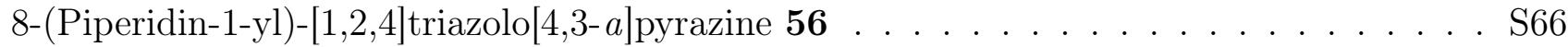

5-(Piperidin-1-yl)-[1,2,4]triazolo[4,3-a]pyrazine-3-d 57 . . . . . . . . . . . . . . S67

8-(Piperidin-1-yl)-[1,2,4]triazolo[4,3-a]pyrazine-3,5- $d_{2} 58 \ldots \ldots$. . . . . . . . . . . S69

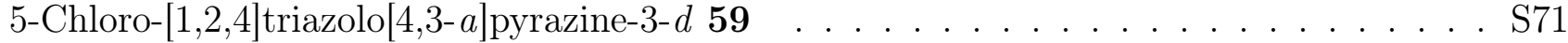

8-(Piperidin-1-yl)-[1,2,4]triazolo[4,3-a]pyrazine-3-d 60 . . . . . . . . . . . . . . S S73

$\begin{array}{lr}\text { S5 X-ray structures } & \text { S75 }\end{array}$

S5.1 Crystal structure determination experimental parameters . . . . . . . . . . . S75

S5.2 X-ray data and structure for 45a . . . . . . . . . . . . . . . . S76

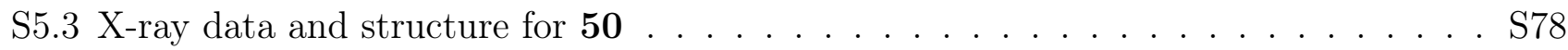

S5.4 X-ray data and structure for $56 \ldots \ldots \ldots$. . . . . . . . . . . . S80

$\begin{array}{ll}\text { References } & \text { S82 }\end{array}$ 


\section{S1 Biological activity experimental details}

\section{S1.1 In Vitro Antiplasmodial Activity}

Evaluation of in vitro Antiplasmodial Activity was conducted at Drug Discovery Unit, University of Dundee following literature procedure. ${ }^{1}$ Cultures of the widely-used malaria reference strain of chloroquinine-sensitive P. falciparum strain 3D7 were maintained in a 5\% suspension of human red blood cells cultured in RPMI 1640 medium supplemented with 0.5\% Albumax II (available from Gibco Life Technologies, San Diego, CA, cat. no. 11021-037), $12 \mathrm{mM}$ sodium bicarbonate, $0.2 \mathrm{mM}$ hypoxanthine, ( $\mathrm{pH} 7.3)$, and $20 \mathrm{mg} / \mathrm{L}$ gentamicin at $37{ }^{\circ} \mathrm{C}$, in a humified atmosphere of $1 \% \mathrm{O} 2,3 \%$ $\mathrm{CO}_{2}$ with a gas balance of nitrogen. Growth inhibition of the P. falciparum cultures was quantified in a 10-point dose response curve with a 1 in 3 dilution series from a top assay concentration of $50 \mathrm{\mu M}$. This 384 well plate based fluorescence assay utilises the binding of SYBRgreen I (Thermo Fisher Scientific/Invitrogen cat. no. S7585) to double stranded DNA, which greatly increases the fluorescent signal at $528 \mathrm{~nm}$ after excitation at $485 \mathrm{~nm}$. Mefloquine was used as a drug control to monitor the quality of the assay $\left(Z^{\prime}=0.6\right.$ to 0.8 , where $Z^{\prime}$ is a measure of the discrimination between the positive and negative controls on a screen plate). Dose-response curves were determined from a minimum of 3 independent experiments. Compound bioactivity was expressed as $\mathrm{IC}_{50}$, the concentration of compound causing $50 \%$ inhibition. $\mathrm{IC}_{50}$ values were determined from a minimum of 3 independent experiments. All data was processed using IDBS ActivityBase ${ }^{\circledR}$ raw data was converted into per cent inhibition through linear regression by setting the high inhibition control as $100 \%$ and the no inhibition control as $0 \%$. Quality control criteria for passing plates were as follows: $Z^{\prime}>0.5, \mathrm{~S}: B>3, \% \mathrm{CV}$ (no inhibition control) $<15$. The formula used to calculate $Z^{\prime}=1-\left(3\left(S_{t}\right.\right.$ Dev $v_{\text {high }}+S t$ Dev $\left.\left._{\text {low }}\right)\right) /\left(A B S\left(\right.\right.$ Mean $_{\text {high }}-$ Mean $\left.\left._{\text {low }}\right)\right)$. All $\mathrm{IC}_{50}$ curve fitting was undertaken using XLFit version 4.2 using Model 205 with the following 4 parametric equation: $y=A+(B-A) /\left(1+(C / x)^{D}\right)$, where $\mathrm{A}=\%$ inhibition at bottom, $\mathrm{B}=\%$ inhibition at top, $\mathrm{C}=$ $\mathrm{IC}_{50}, \mathrm{D}=$ slope, $\mathrm{x}=$ inhibitor concentration and $\mathrm{y}=\%$ inhibition. If curve did not reach $100 \%$ of inhibition, B was fixed to 100 only when at least $50 \%$ of inhibition was reached.

\section{S1.2 KINOMEscan ${ }^{\circledR}$ Assay}

Evaluation was performed following literature procedure. ${ }^{2}$ All compounds were dissolved and stored at $-20{ }^{\circ} \mathrm{C}$ as $10 \mathrm{mM}$ DMSO stocks. Compounds were screened at a single concentration of $1 \mu \mathrm{M}$ using binding assays as described previously. ${ }^{3,4}$ Briefly, extracts containing kinases (expressed either as T7 phage fusion proteins or DNA-tagged) are incubated with an immobilized bait compound (known inhibitor like staurosporine) and the test compound (at $1 \mu \mathrm{M}$ ). Test compounds that displace the immobilized bait compound are detected by quantitative PCR. The results are expressed as the percentage of kinase bound to the bait compound bead relative to a DMSO control (\% control). Compounds with high affinity have \% control of $0-1$ whereas compounds with lower affinity have higher scores. 


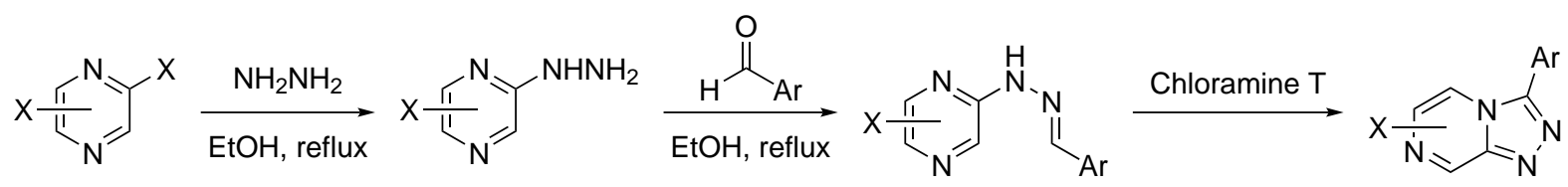

Figure S1: Synthetic pathway for studied $[1,2,4]$ triazolo[4,3-a]pyrazines.

\section{S3 Mechanism of transformation that leads to the ring open- ing product 50}<smiles>COc1ccc(-c2nnc3cncc(I)n23)cc1</smiles>

$45 n$

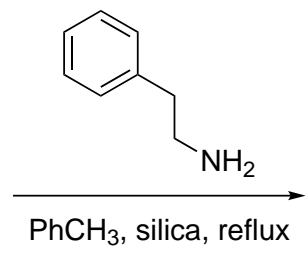

, silica, reflux<smiles>COc1ccc(-c2nc(-c3nccn3CCc3ccccc3)n[nH]2)cc1</smiles>

50

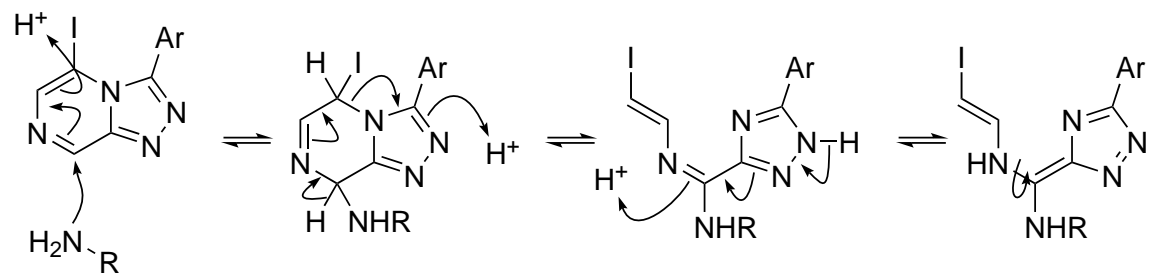

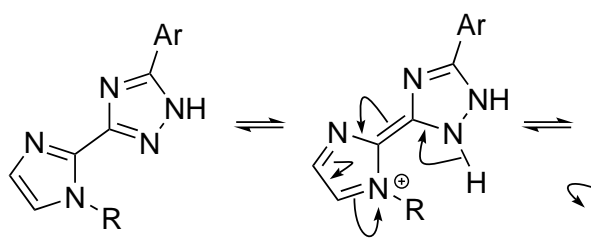

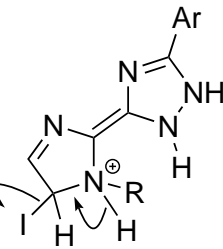<smiles>C=CCI</smiles>

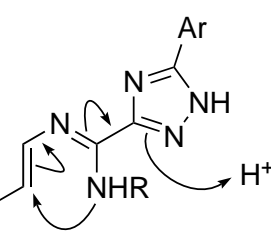<smiles></smiles>

Figure S2: Detailed mechanism of transformation that leads to the ring opening product 50. This mechanism involves a 5-endo-trig cyclisation though this type of cyclisation is disfavoured according to Baldwin's rules. ${ }^{5}$ Examples of disfavoured reactions are known and were reported by Baldwin. ${ }^{6}$ Such cyclisations can be made more feasible if they involve stabilisation of an intermediate anion (on one of the carbons of the ring double bond after the first step) by adjacent groups, such as the C-I sigma star ("exocyclic hyperconjugation") or by virtue of the charge being part of an extended aromatic system involving the adjacent ring. ${ }^{7}$ As for other apparent violations of Baldwin's rules, there is no competing ring closure pathway available.

\section{S4 NMR spectra of compounds}




\section{2-Bromo-6-hydrazinylpyrazine S2}

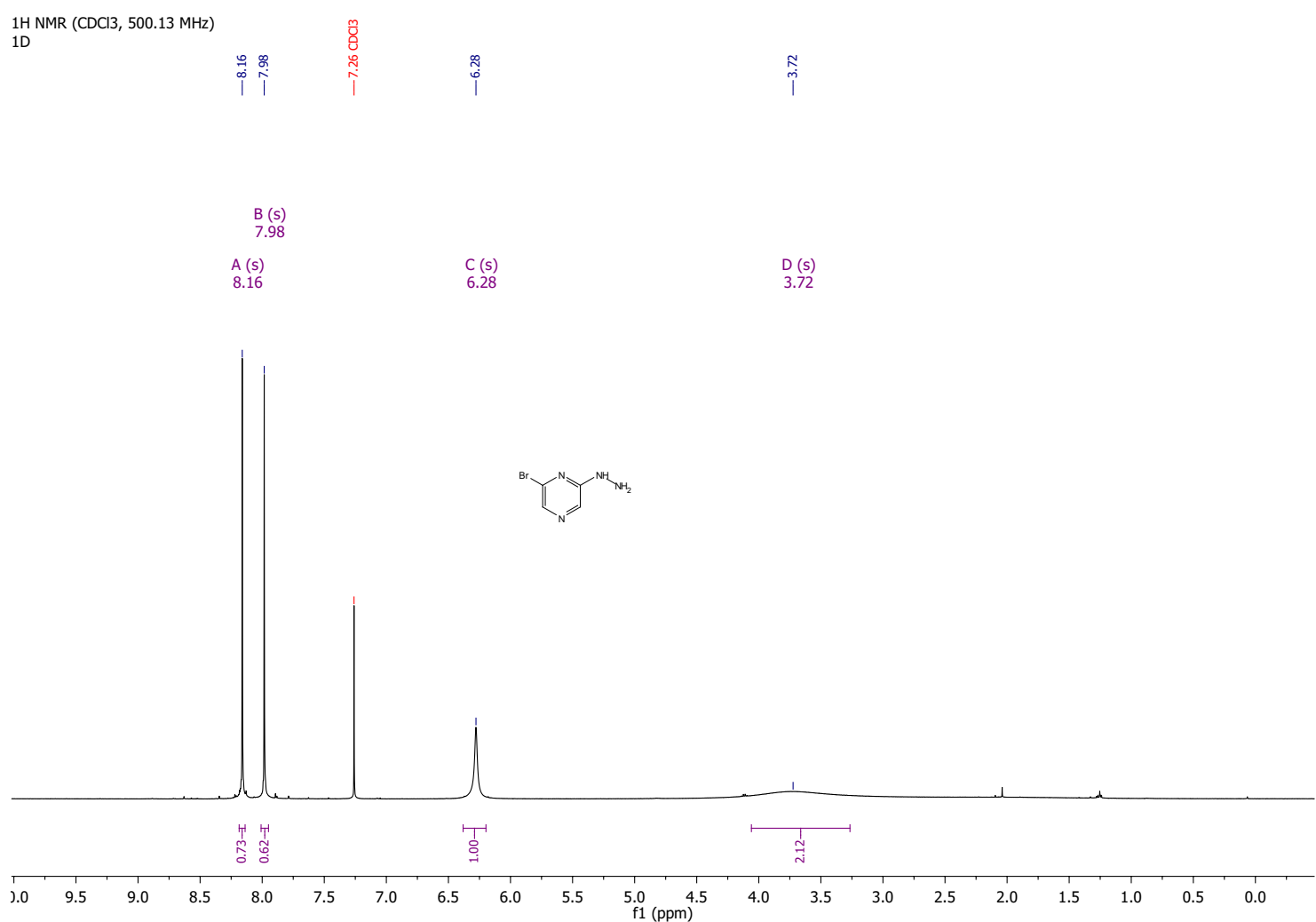

$13 \mathrm{C} \mathrm{NMR}(\mathrm{CDCl} 3,125.77 \mathrm{MHz})$
$1 \mathrm{D}$
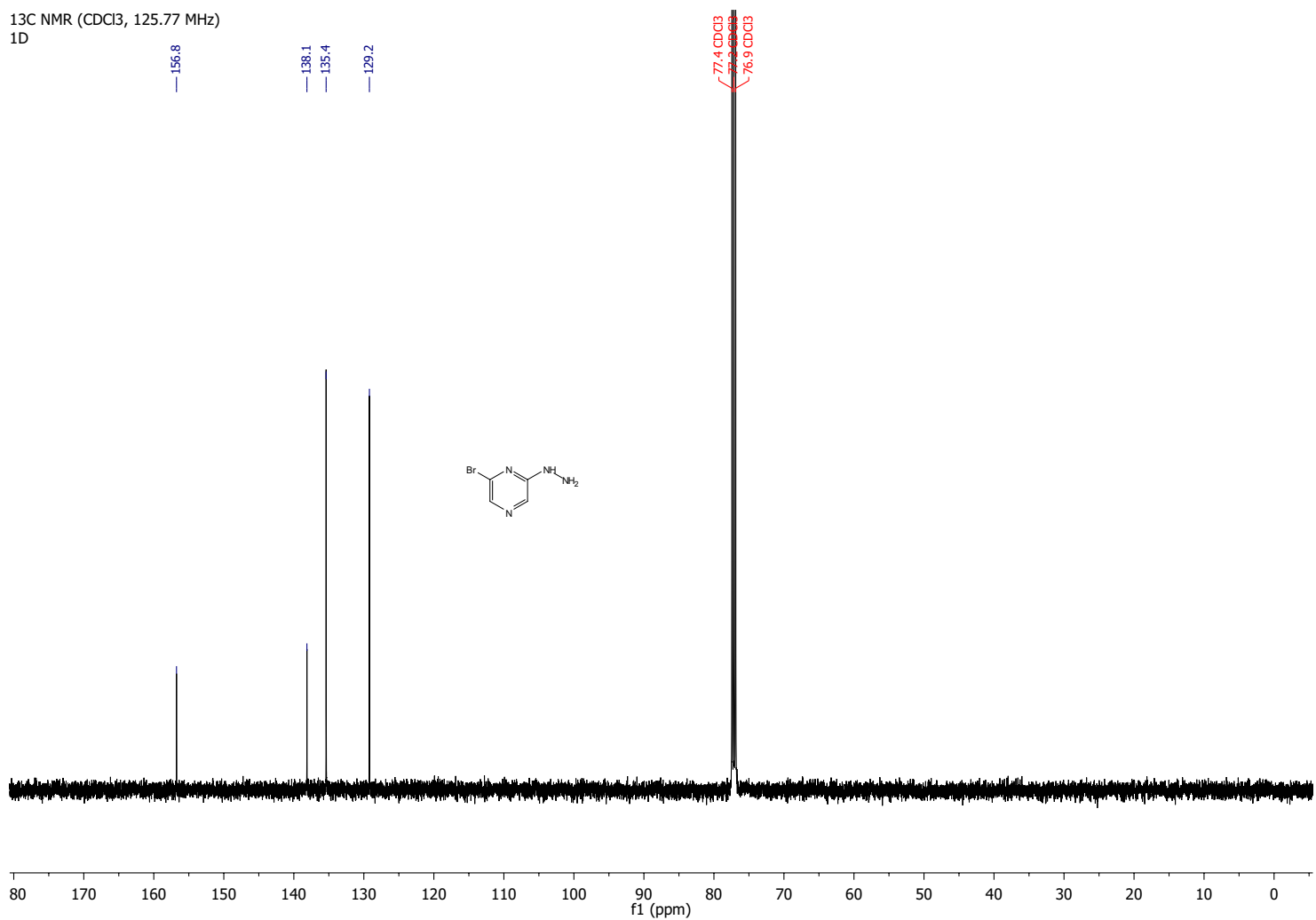


\section{2-Iodo-6-hydrazinylpyrazine S3}
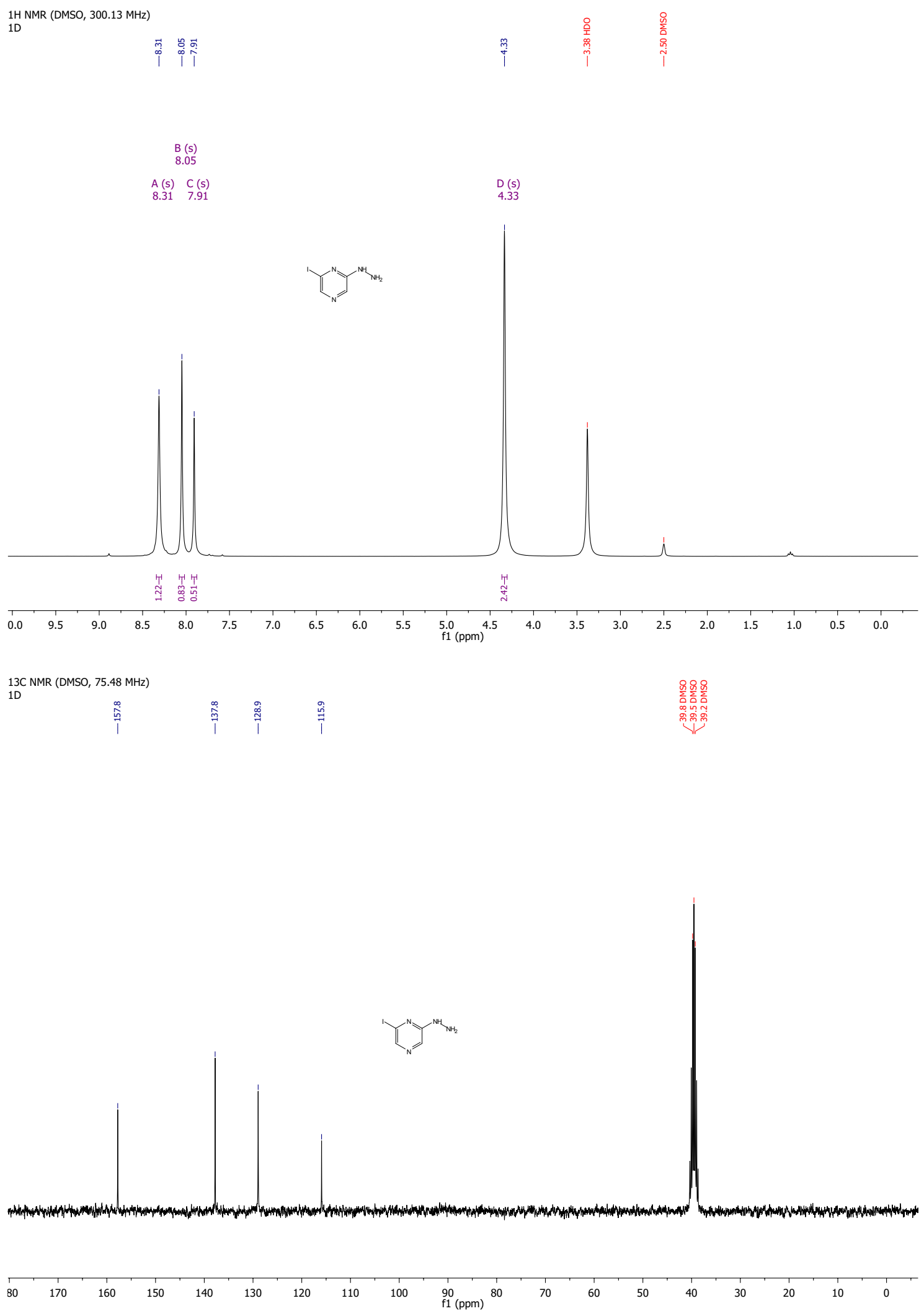


\section{2-Chloro-3-hydrazinylpyrazine S4}
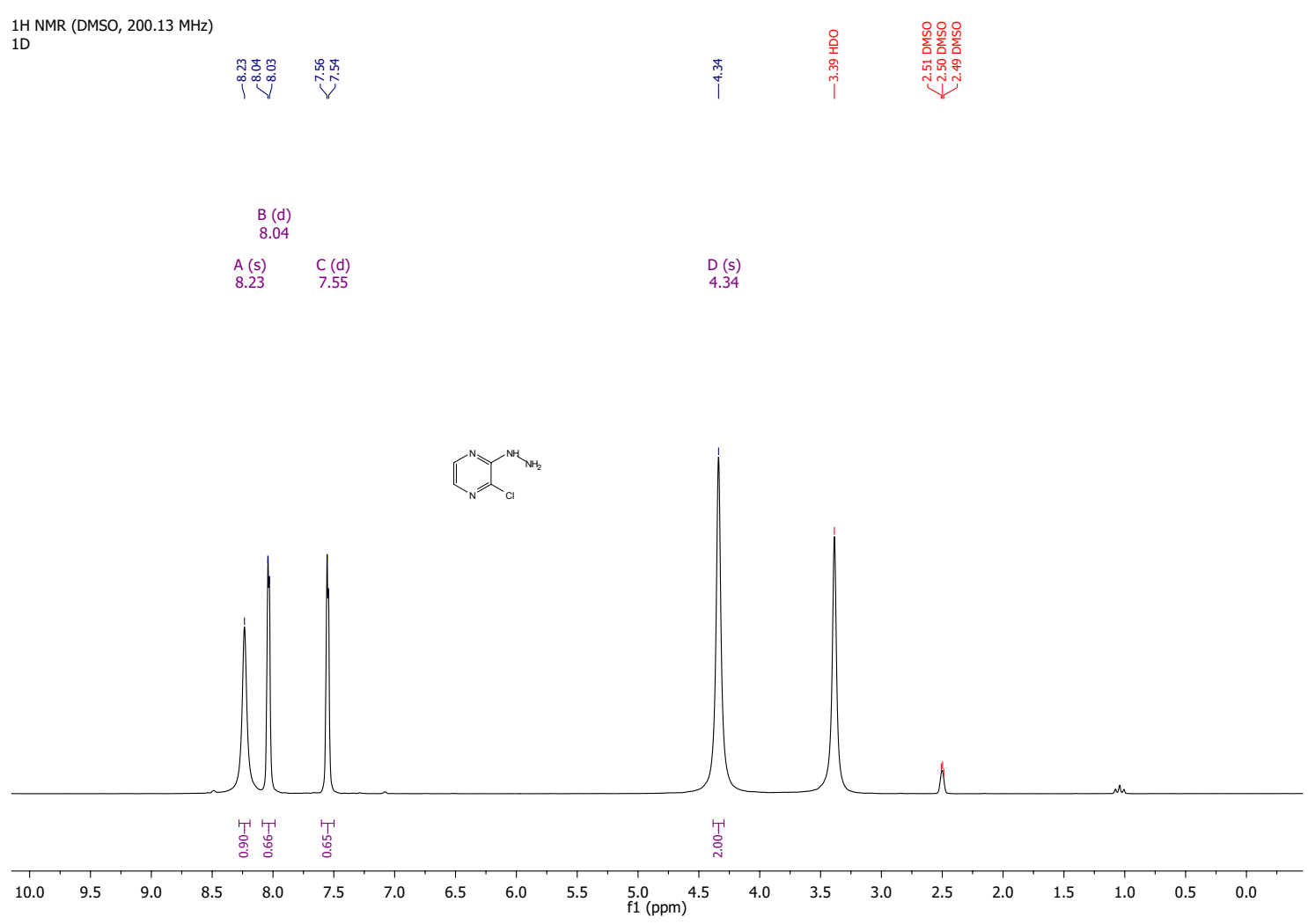

13C NMR (DMSO, $50.33 \mathrm{MHz}$ )
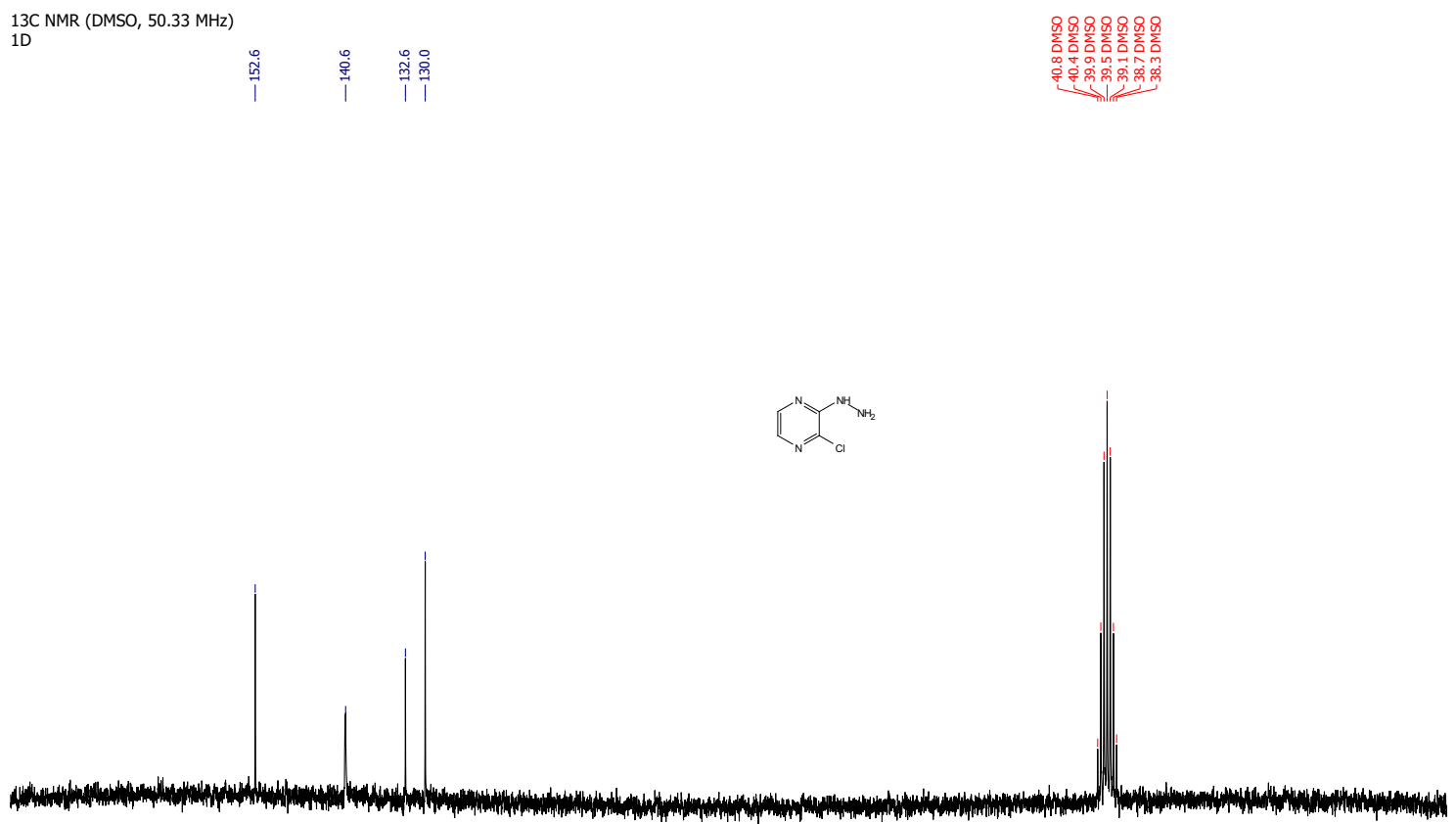

2-Chloro-5-hydrazinylpyrazine S5 

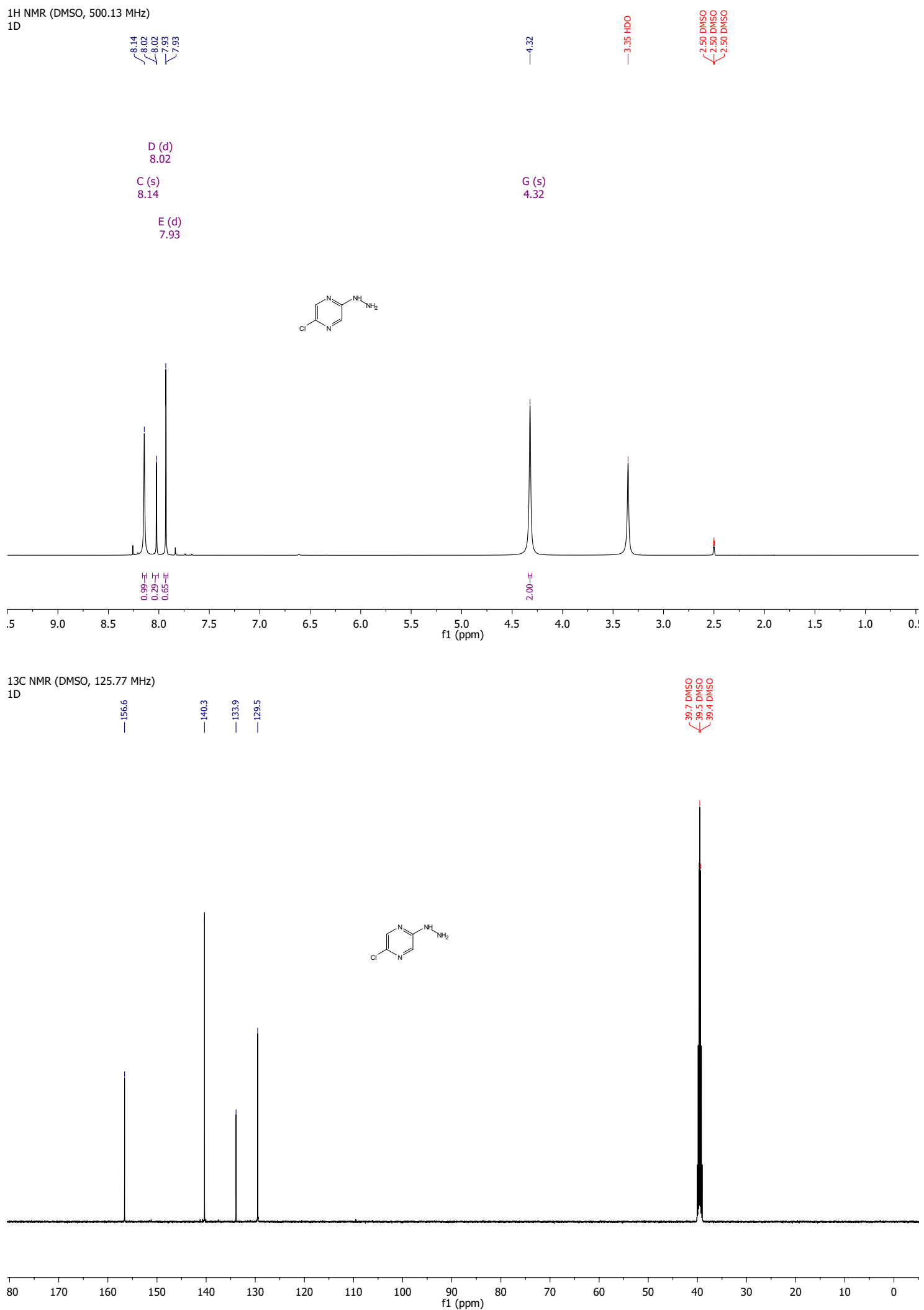
5-Chloro-3-(4-(difluoromethoxy)phenyl)-[1,2,4]triazolo[4,3-a]pyrazine 16

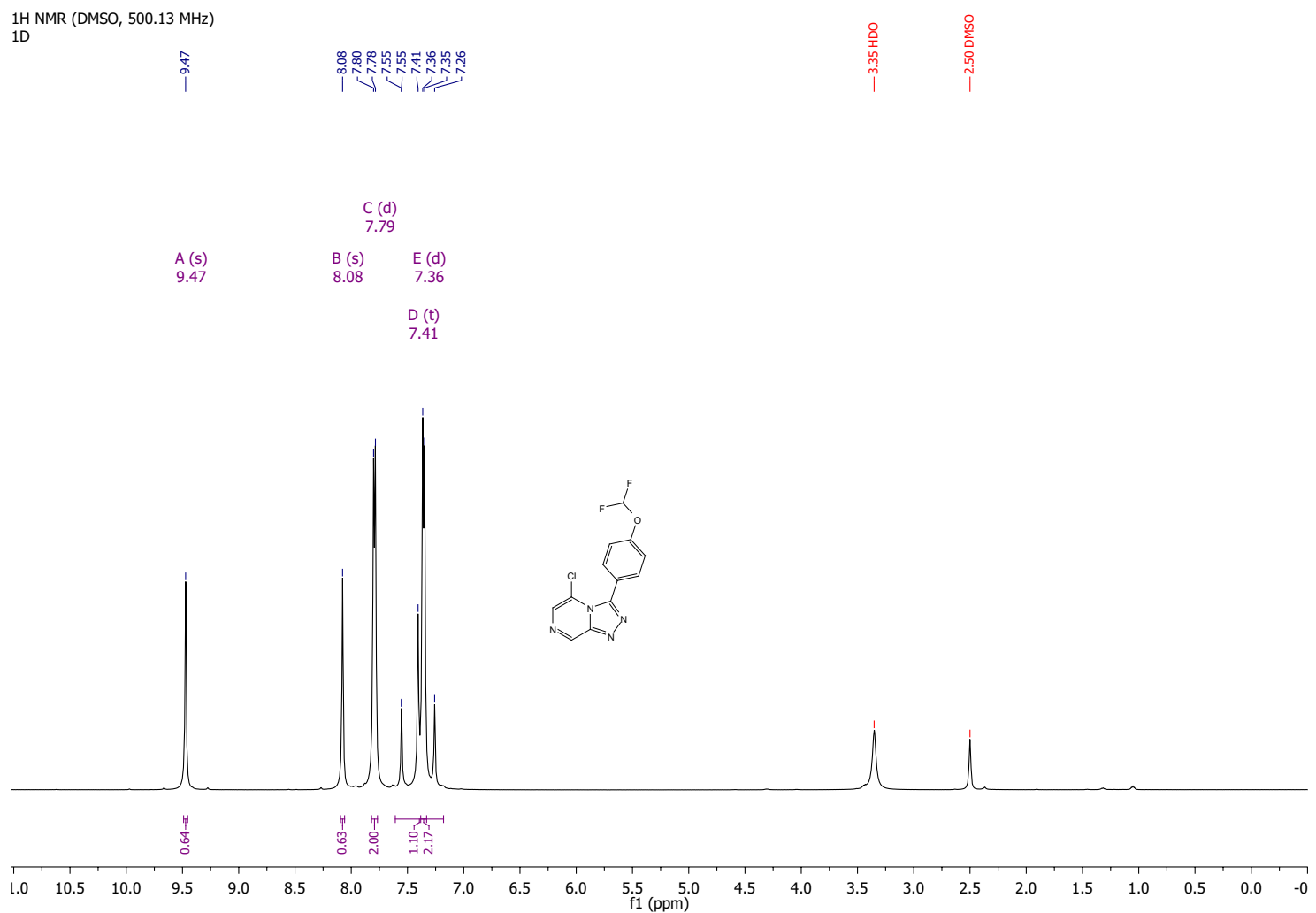

13C NMR (DMSO, $125.77 \mathrm{MHz}$ )

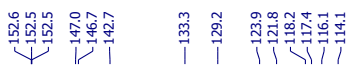

A (m)
152.5

B (t)

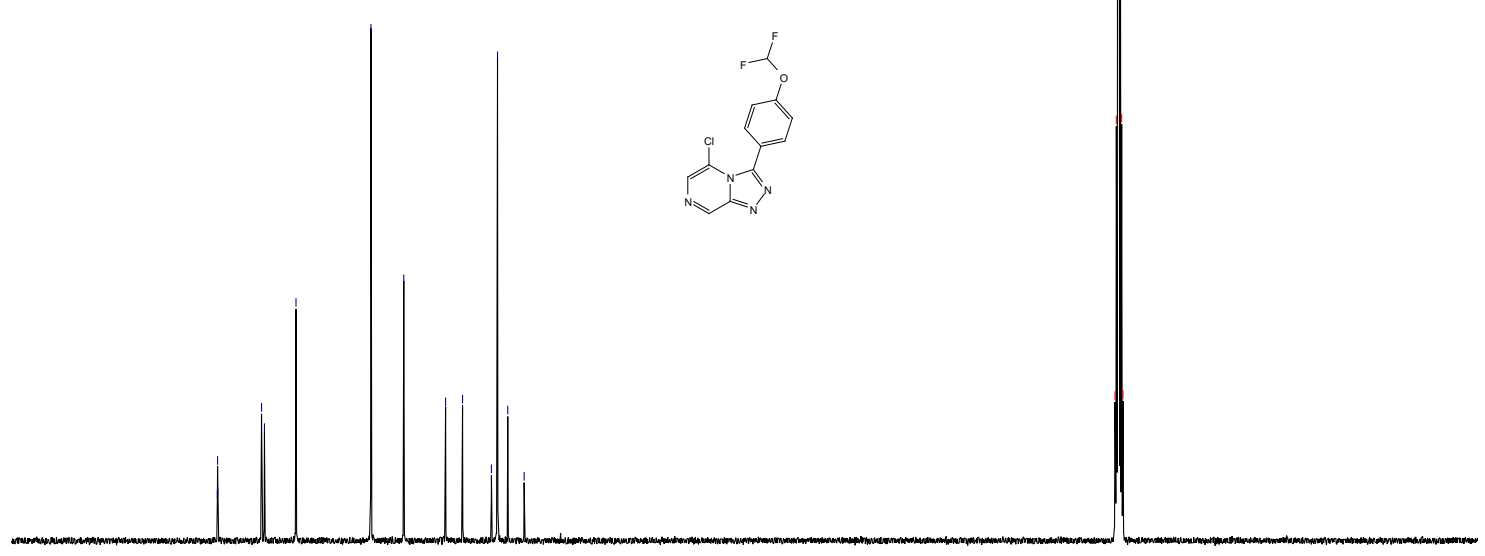

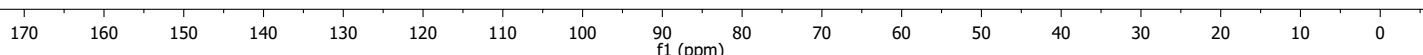


3-(4-(Difluoromethoxy)phenyl)-5-(phenethylthio)-[1,2,4]triazolo[4,3-a]pyrazine 17

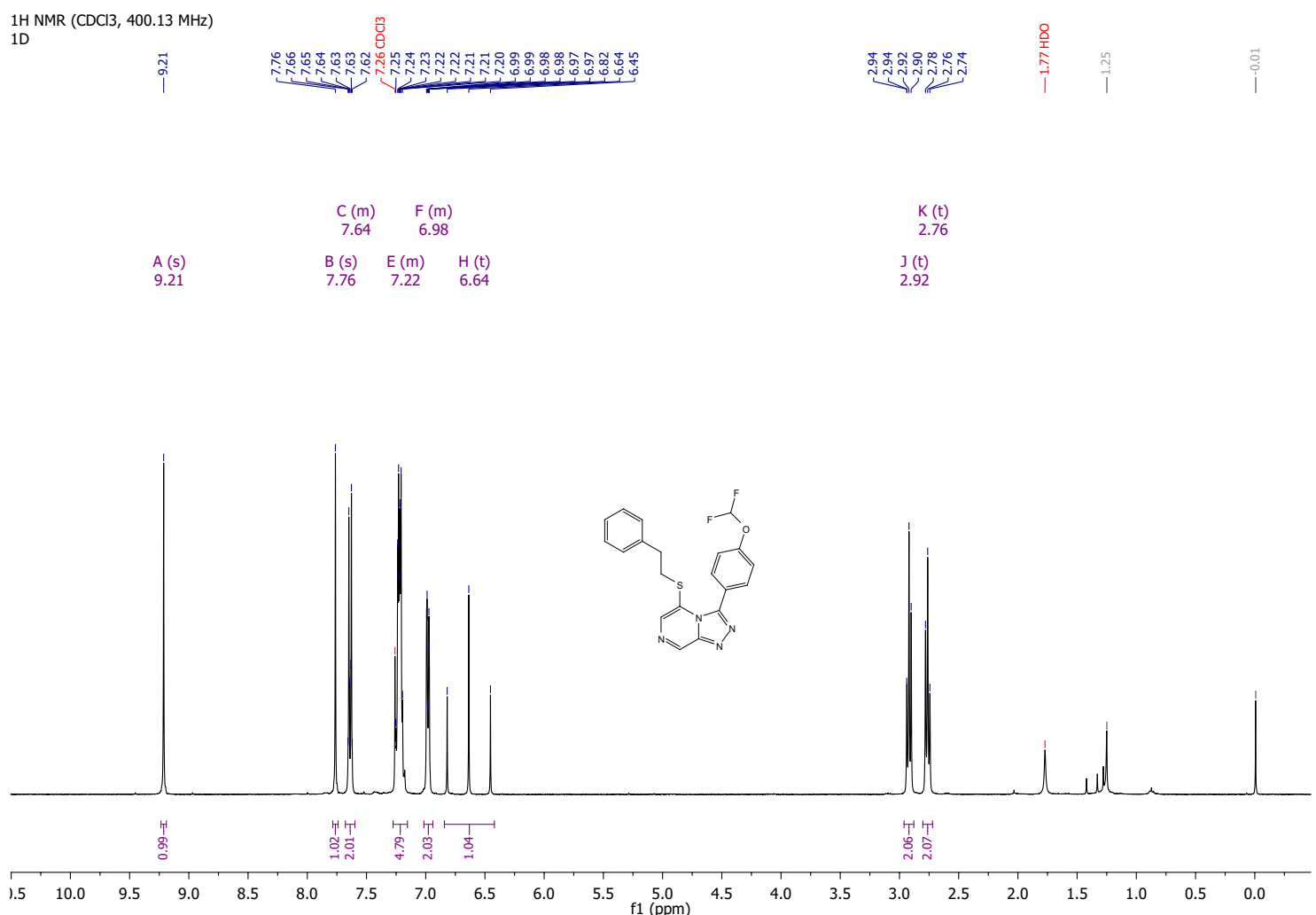

$13 \mathrm{C} \mathrm{NMR} \mathrm{(CDCl3,} 100.62 \mathrm{MHz})$
$1 \mathrm{D}$

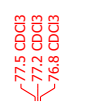

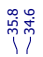

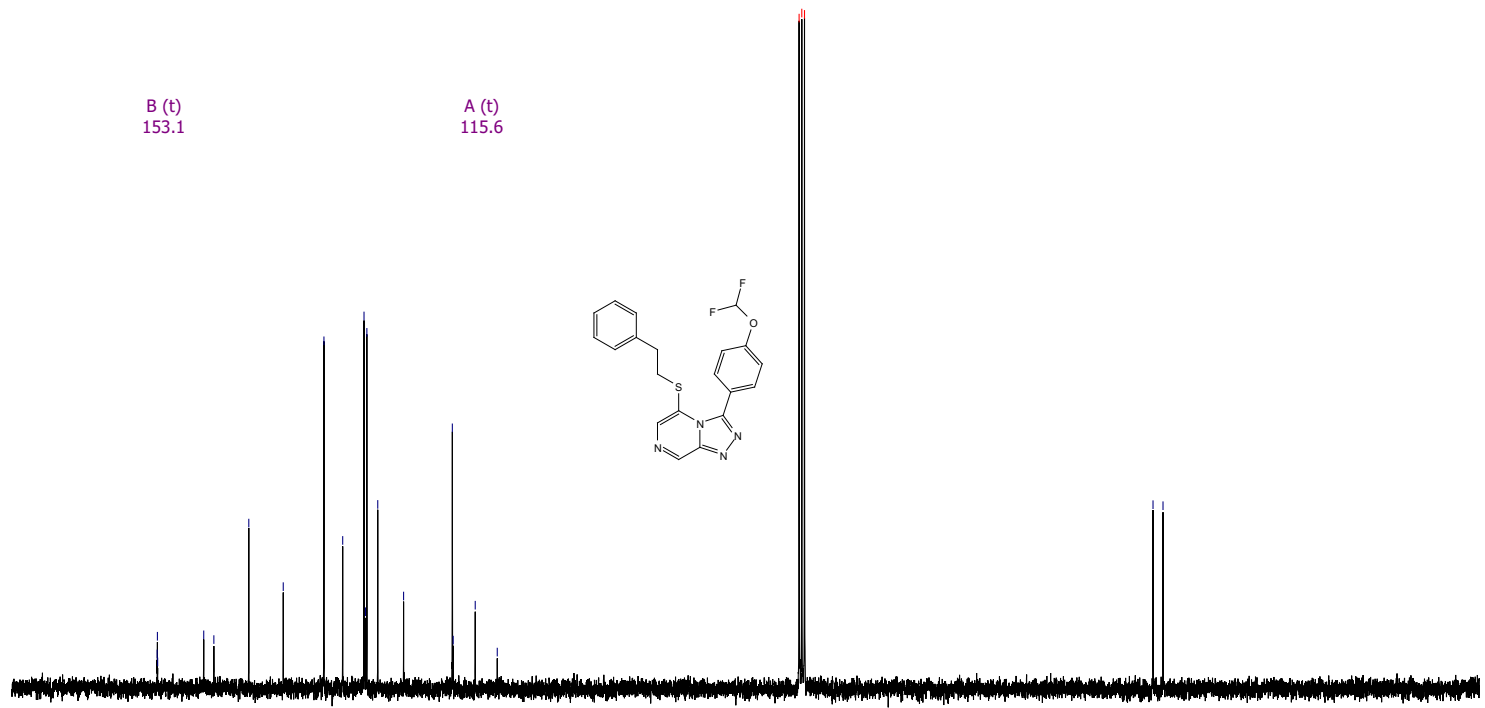

\begin{tabular}{lllllllllllllllll}
1 & 1 & 1 & 1 & 1 & 1 & 1 & 1 & 1 & 1 & 1 & 1 & 1 & 1 & 1 & 1 & 1 \\
\hline
\end{tabular} 
3-(4-(Difluoromethoxy)phenyl)-8-(phenethylthio)-[1,2,4]triazolo[4,3-a]pyrazine 18

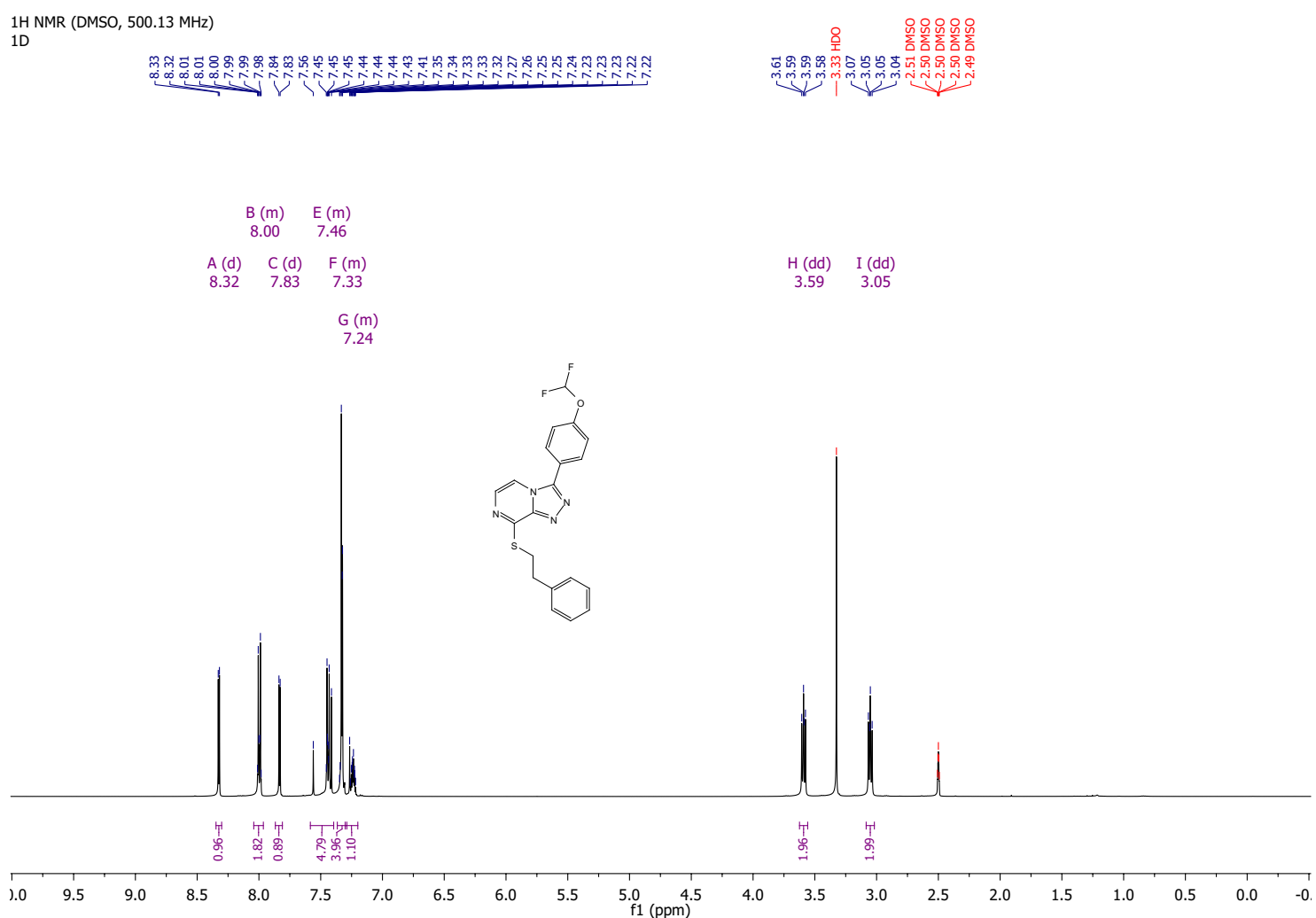

13C NMR (DMSO, $125.77 \mathrm{MHz})$
1D

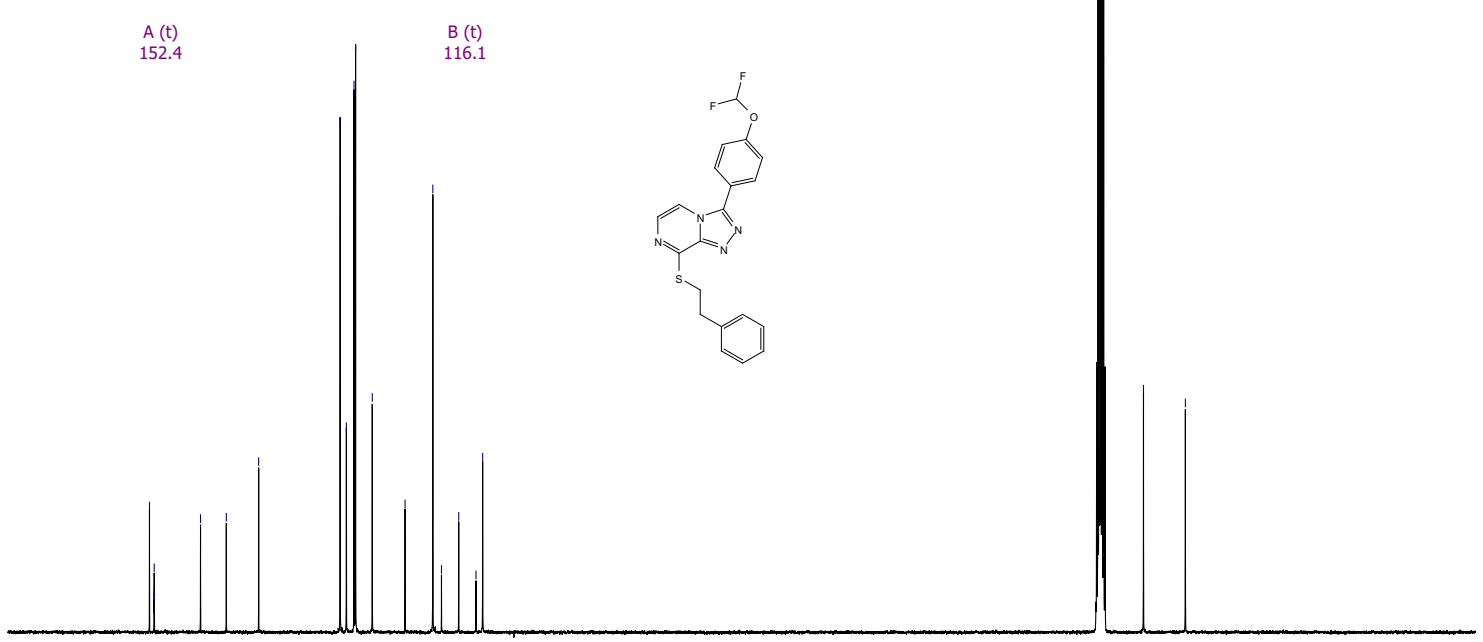




\section{8-Chloro-[1,2,4] triazolo[4,3-a]pyrazine 25}

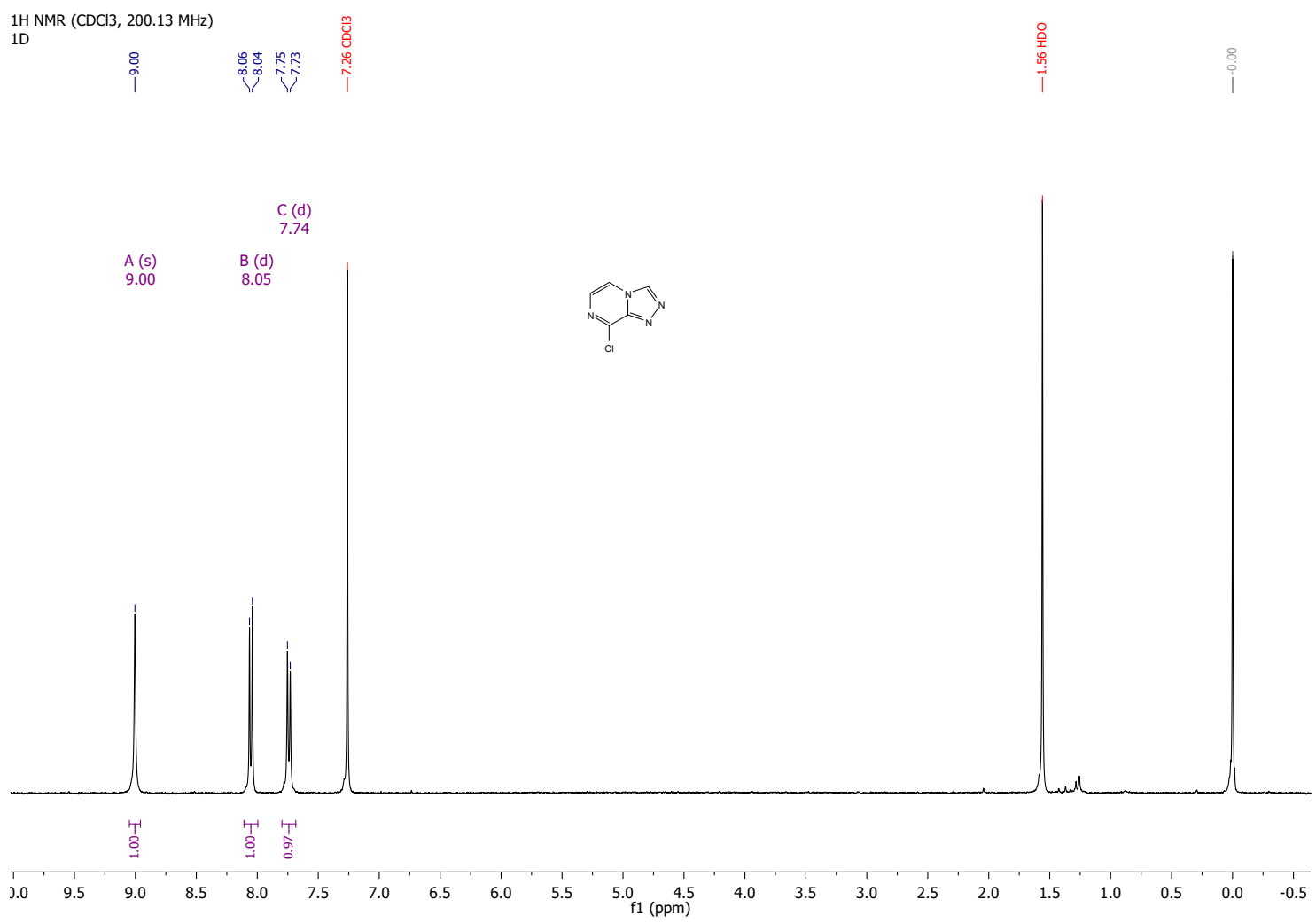


8-Chloro-3-phenyl-[1,2,4] triazolo[4,3-a]pyrazine 26
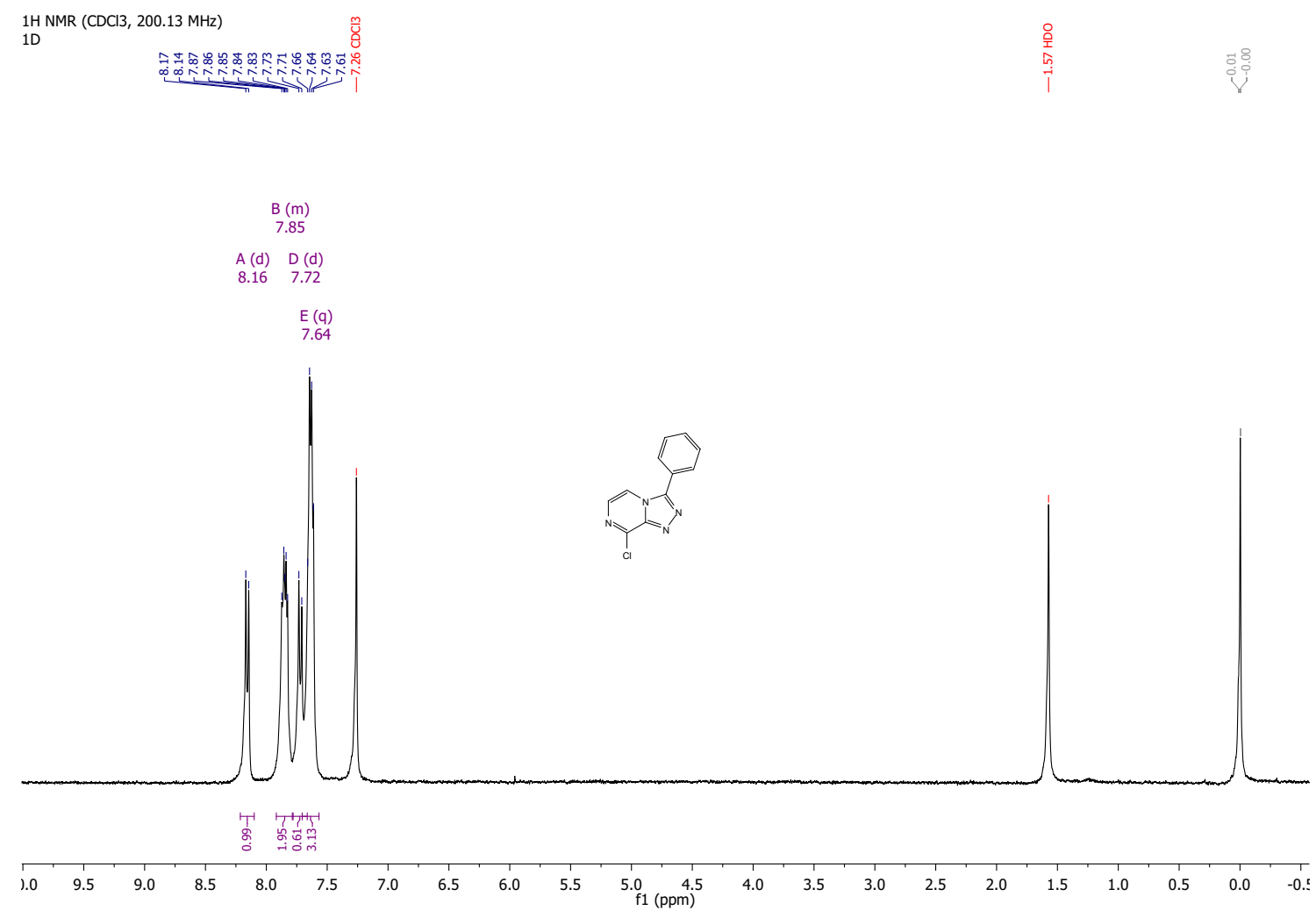
8-Chloro-3-(4-nitrophenyl)-[1,2,4]triazolo[4,3-a]pyrazine 27

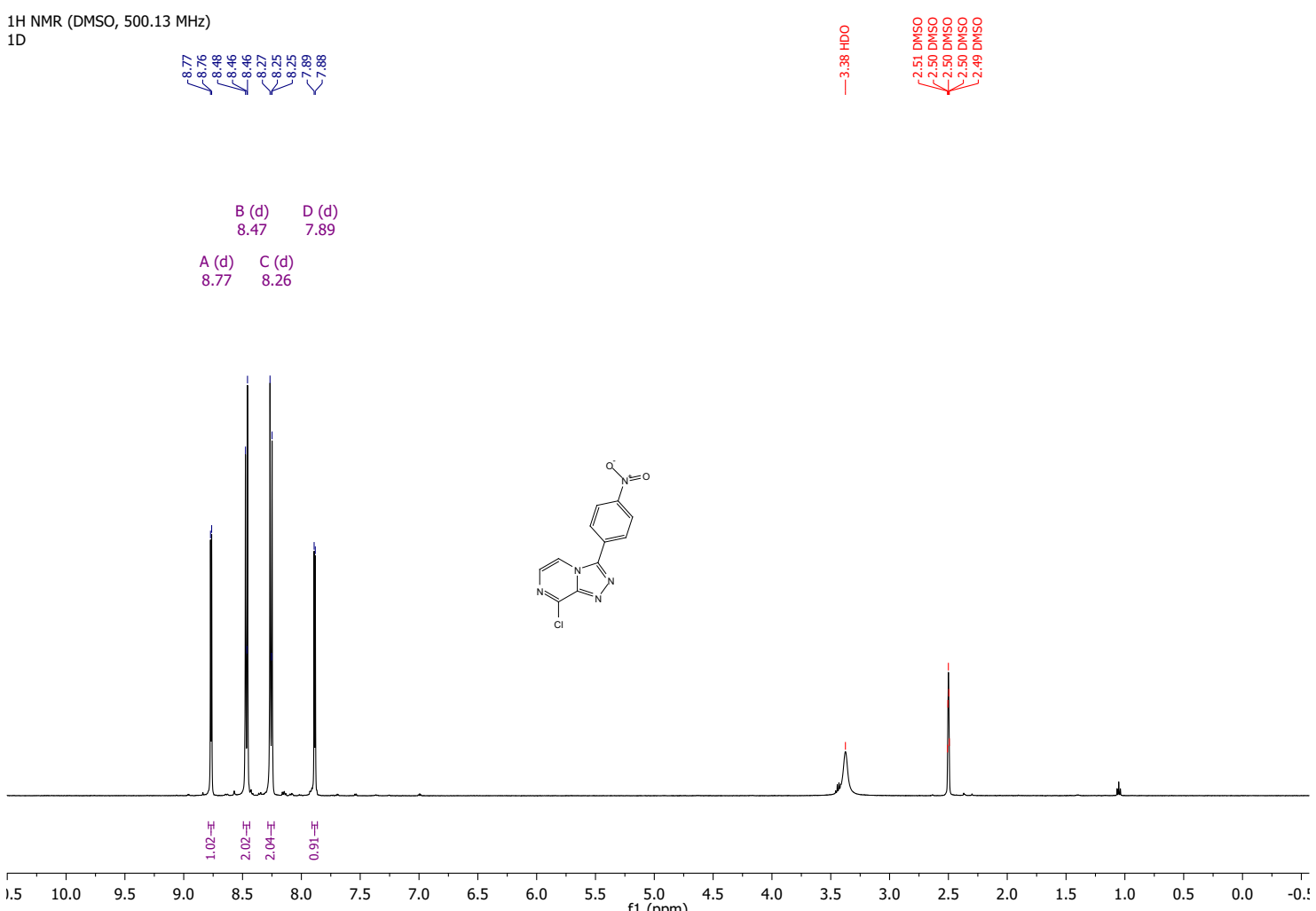

13C NMR (DMSO, $125.77 \mathrm{MHz})$

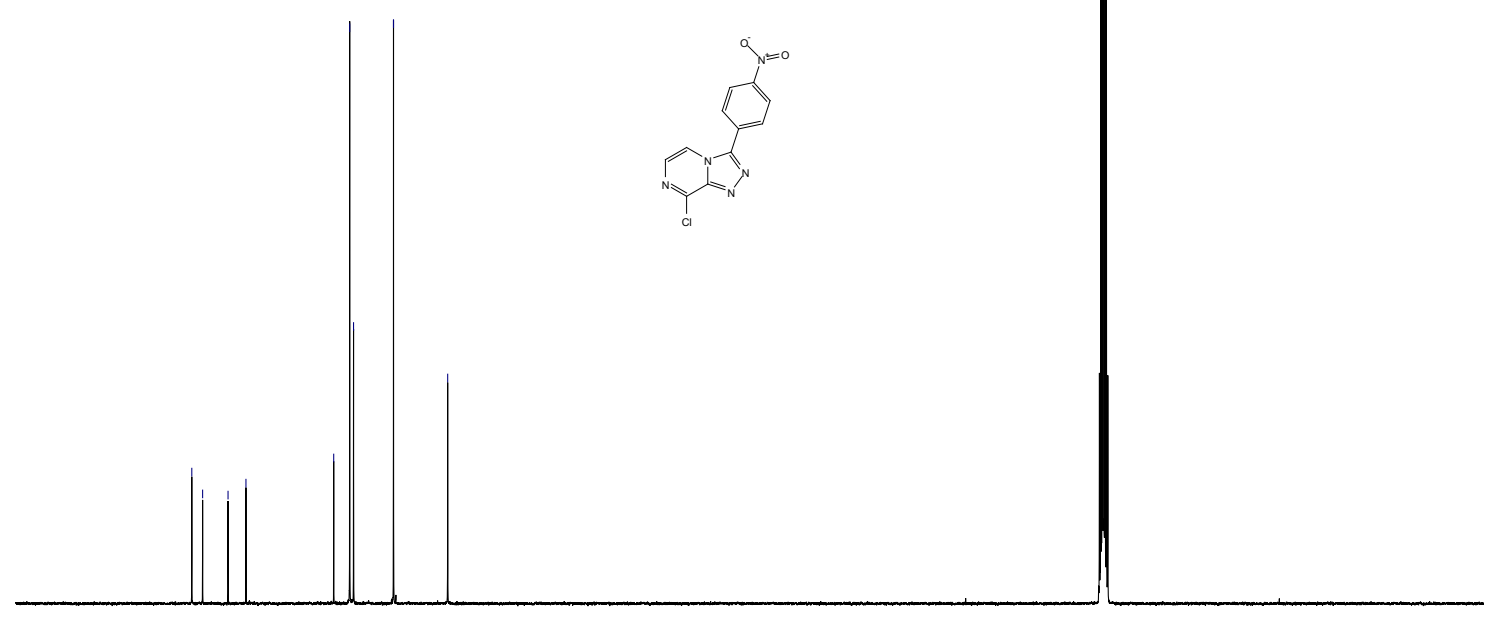

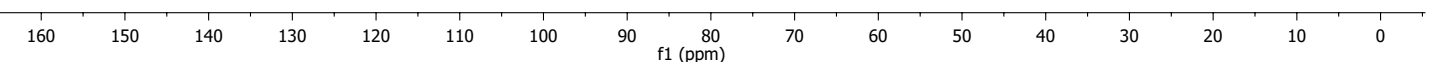


8-Phenethoxy-[1,2,4]triazolo[4,3-a]pyrazine 28
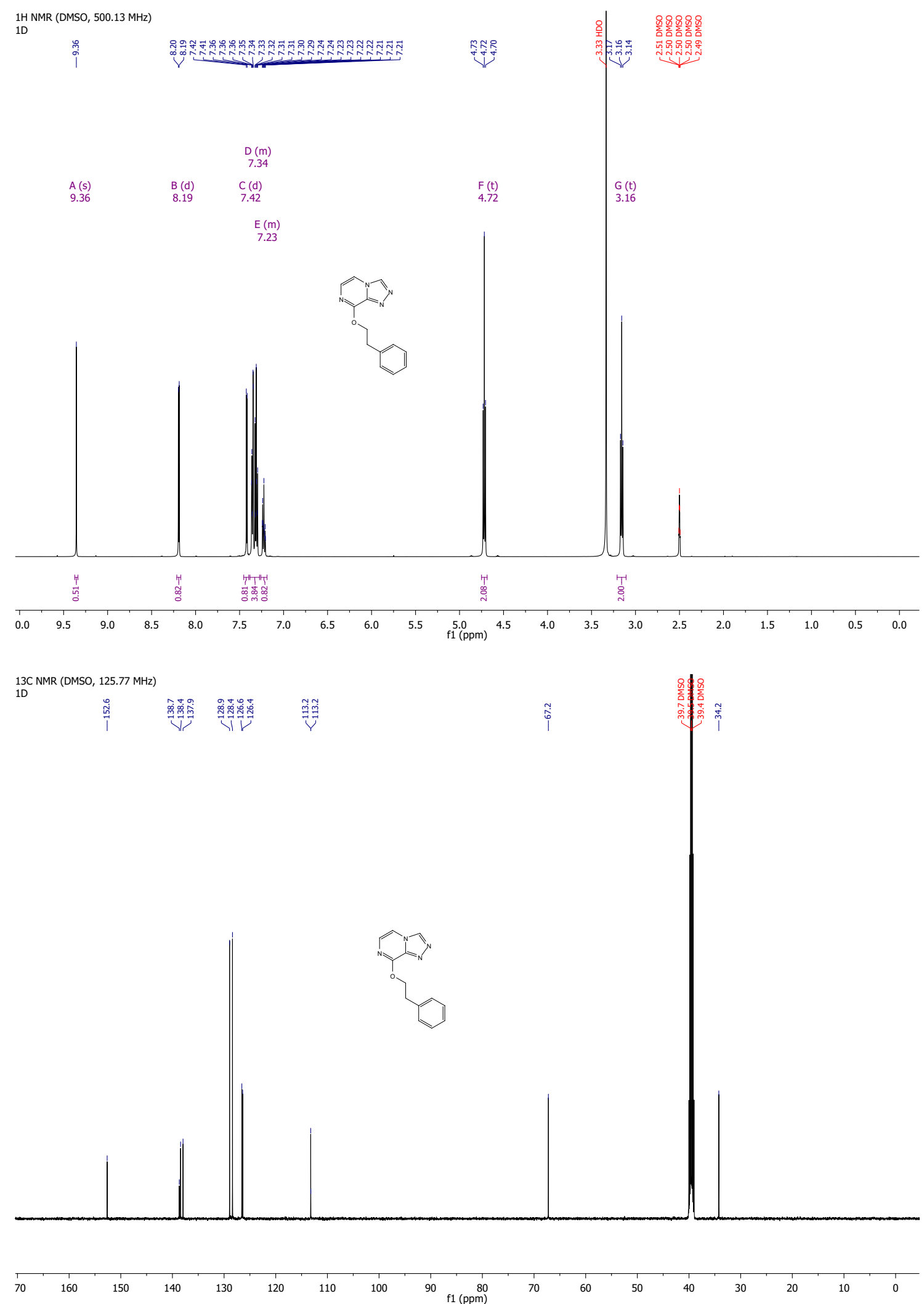
8-(Phenethylthio)-[1,2,4]triazolo[4,3-a]pyrazine 29
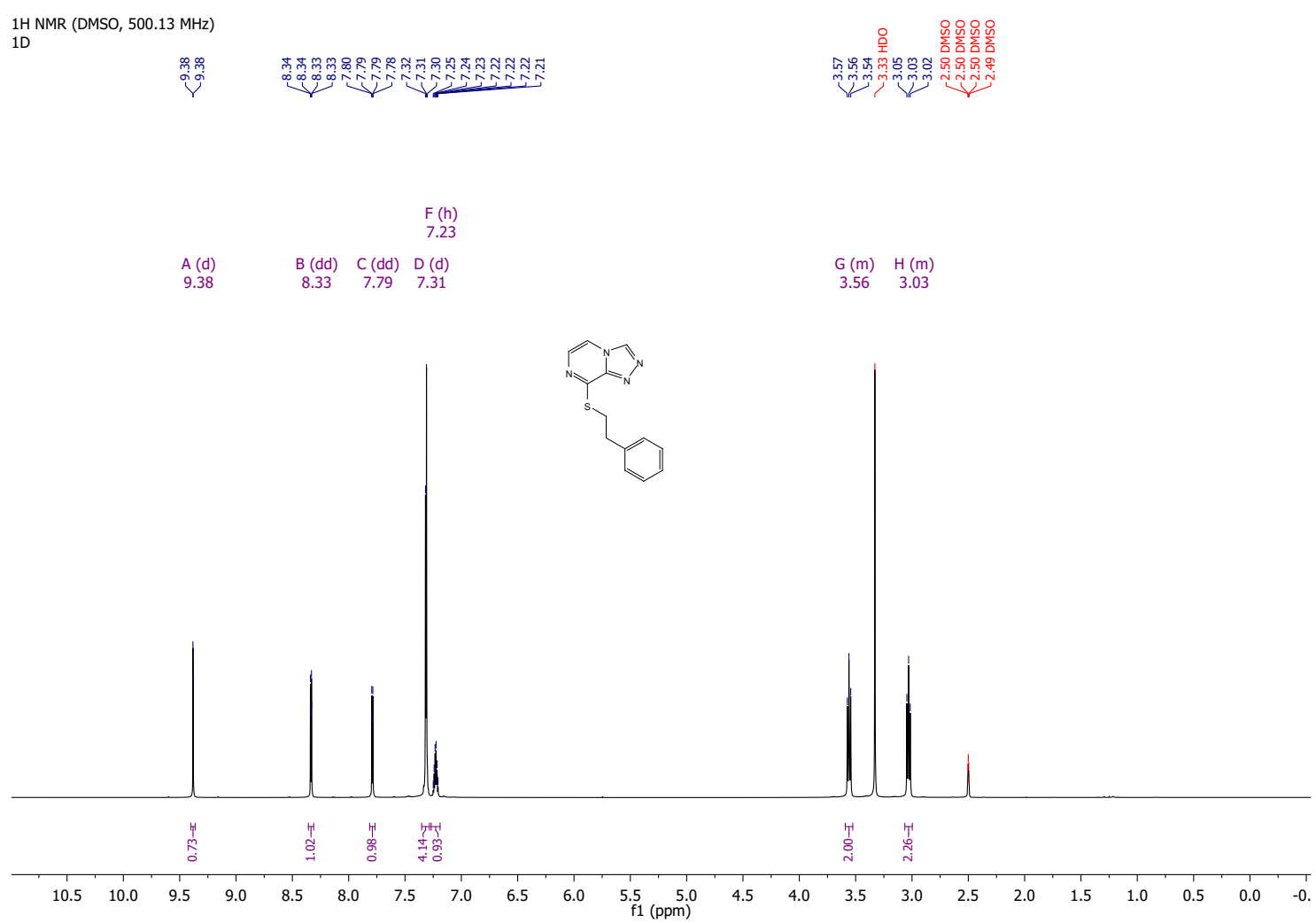

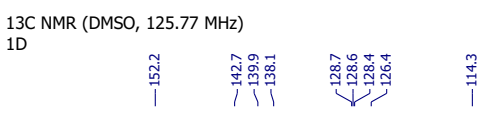

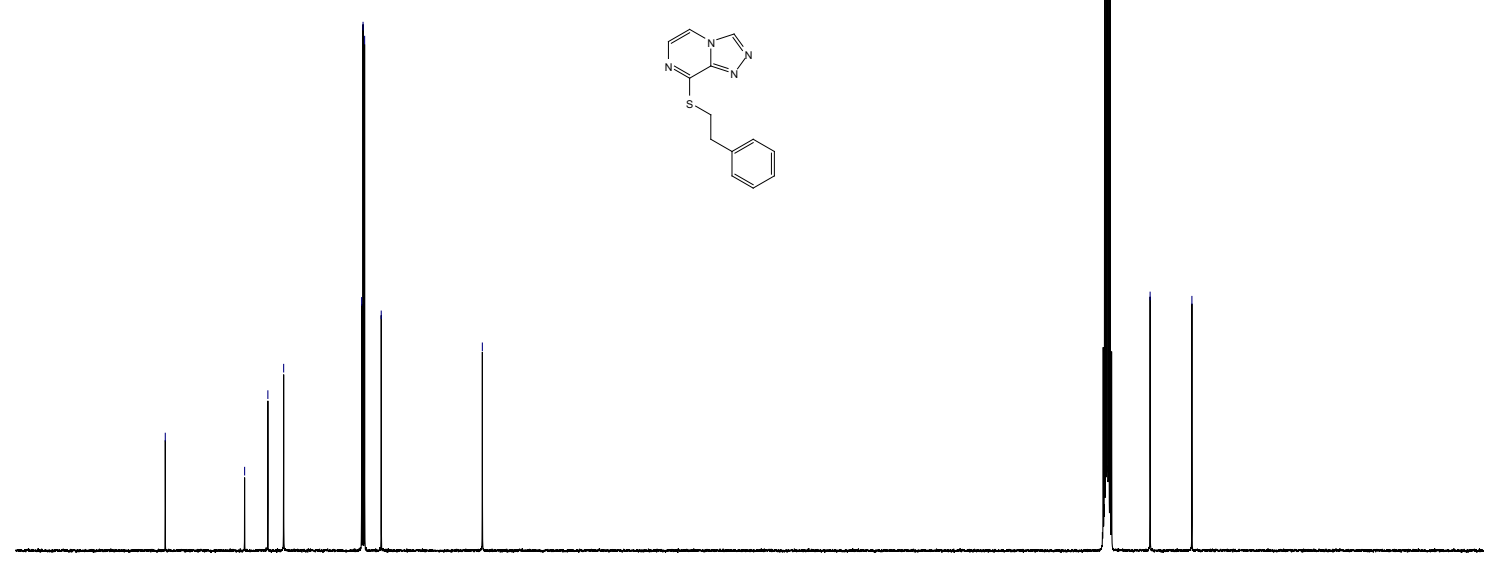

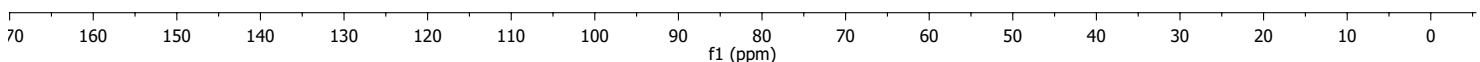


$N$-Phenethyl-[1,2,4]triazolo[4,3-a]pyrazin-8-amine 30

1H NMR (DMSO,
$1 \mathrm{D}$
50.13 MHz)
$\substack{9 \\ 0}$
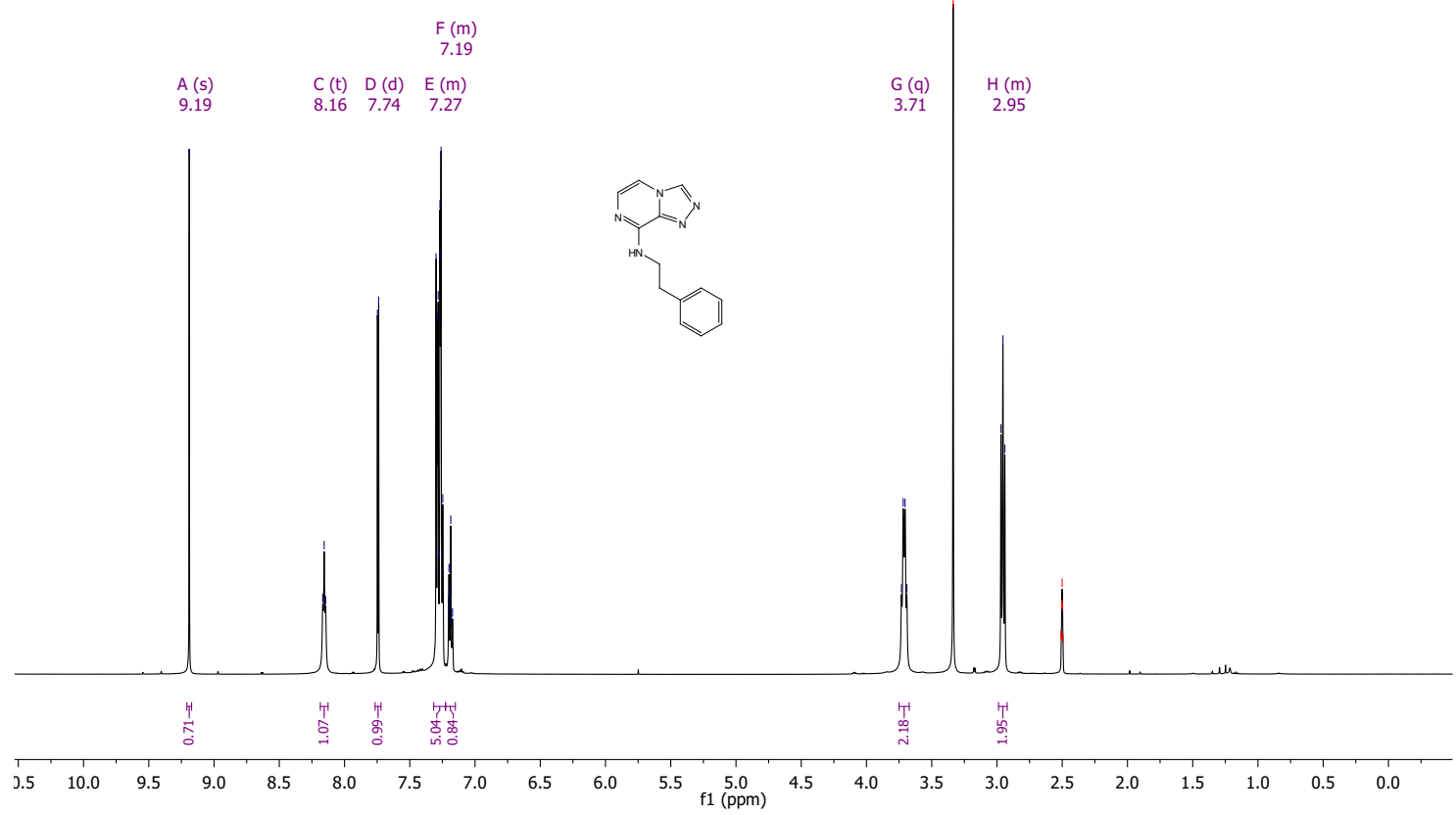

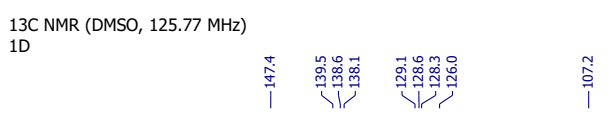

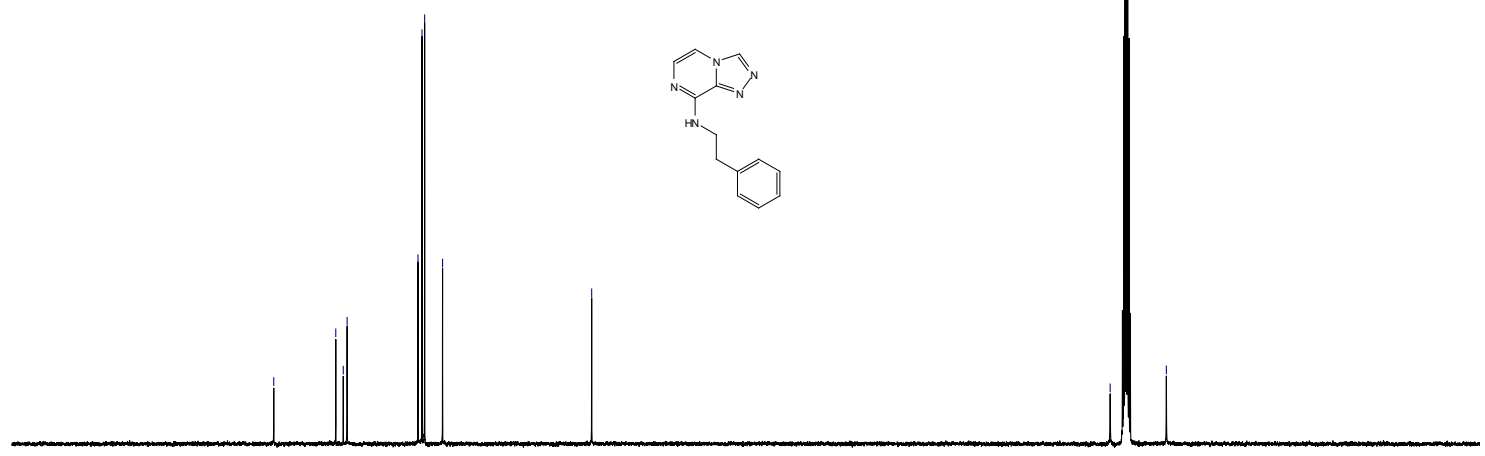

80

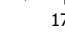

170

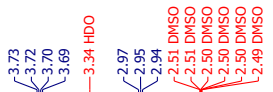

\begin{tabular}{c|c} 
& \\
G (q) & $H(m)$ \\
3.71 & 2.95
\end{tabular}

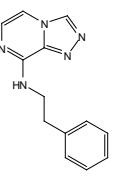

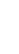

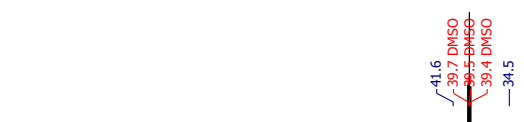


8-Phenethoxy-3-phenyl-[1,2,4] triazolo[4,3-a]pyrazine 31

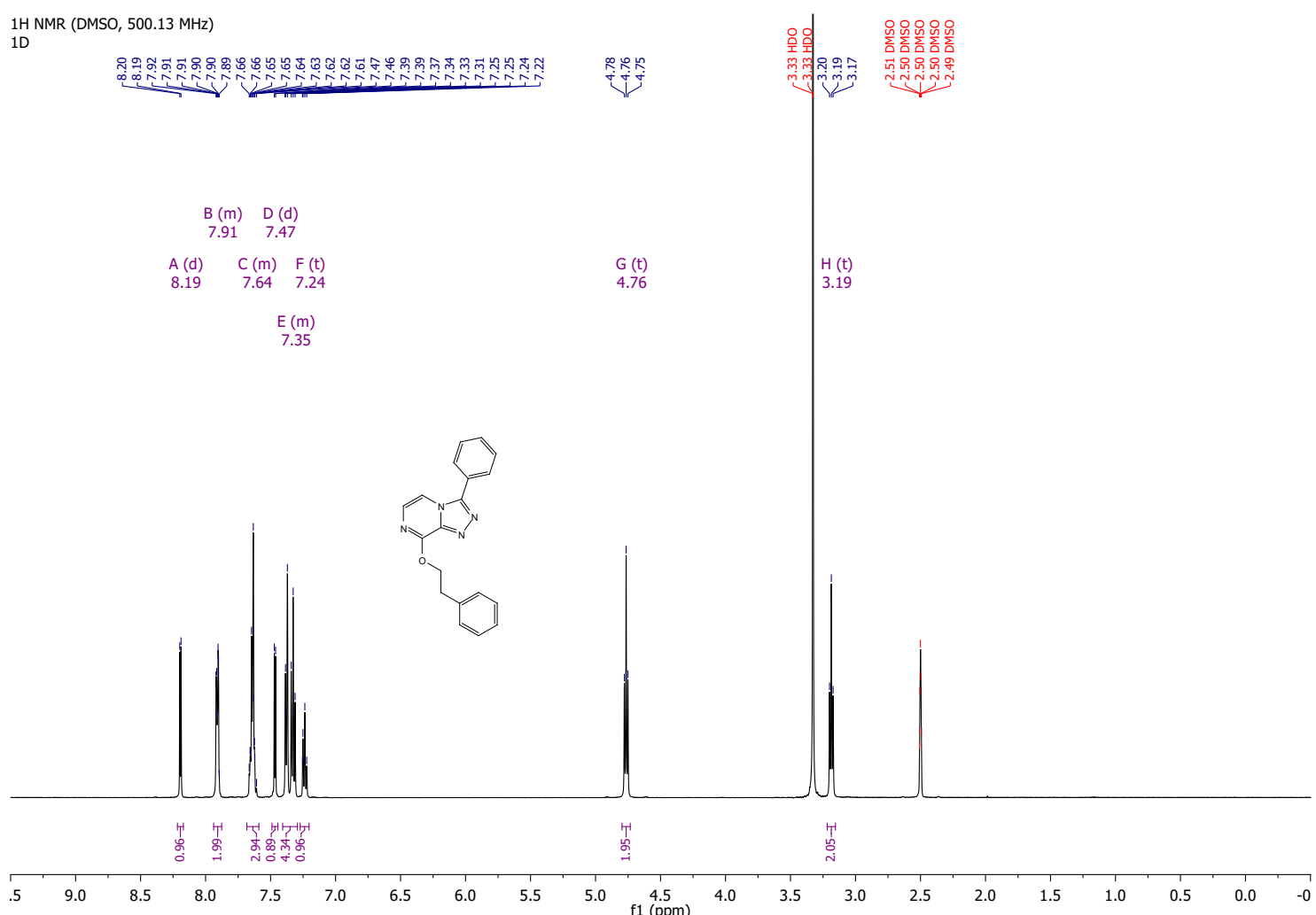

$13 \mathrm{C}$ NMR (DMSO, $125.77 \mathrm{MHz})$
$1 \mathrm{D}$
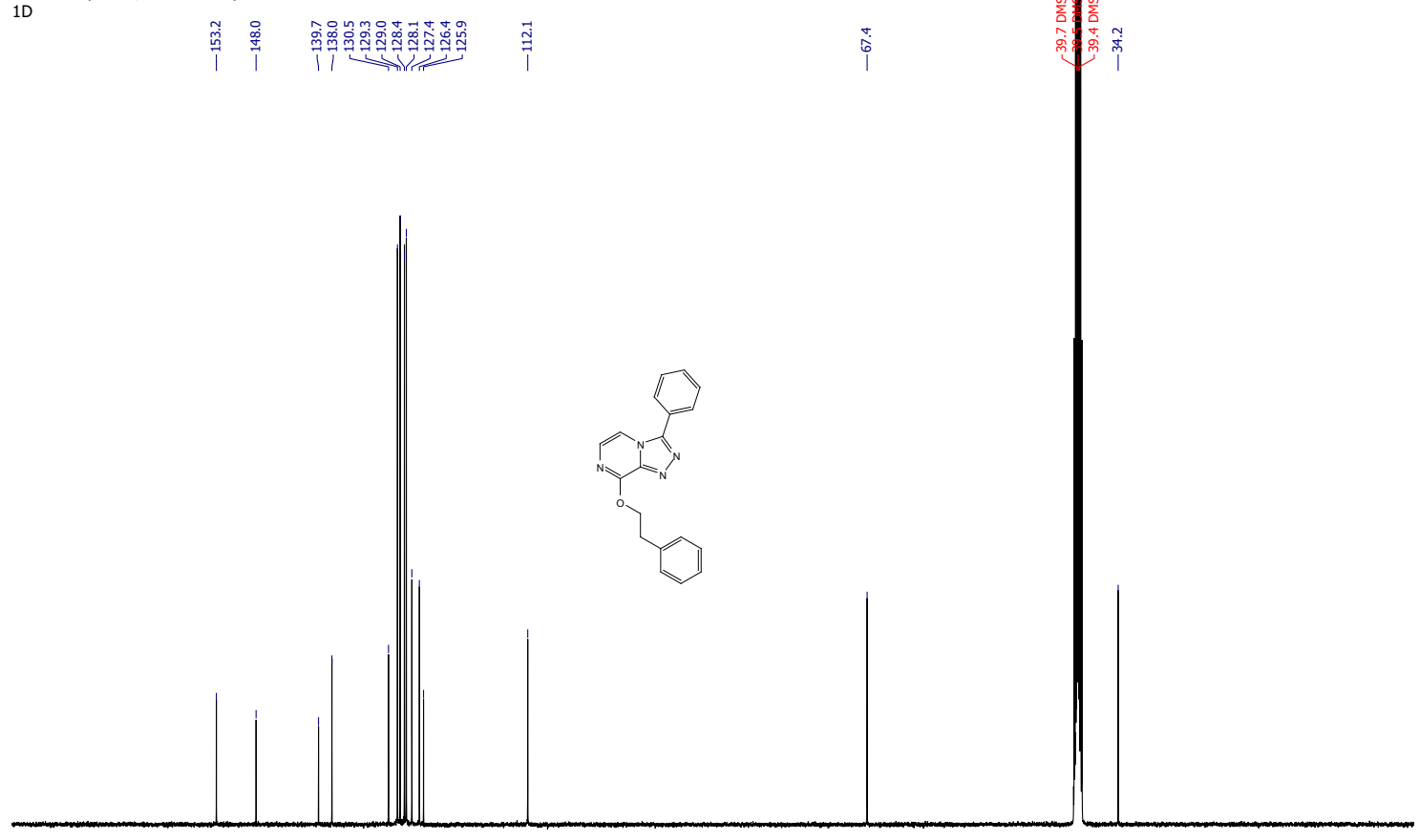

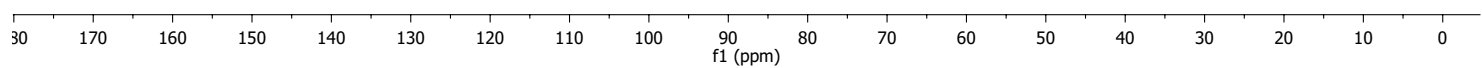


8-(Phenethylthio)-3-phenyl-[1,2,4] triazolo[4,3-a]pyrazine 32

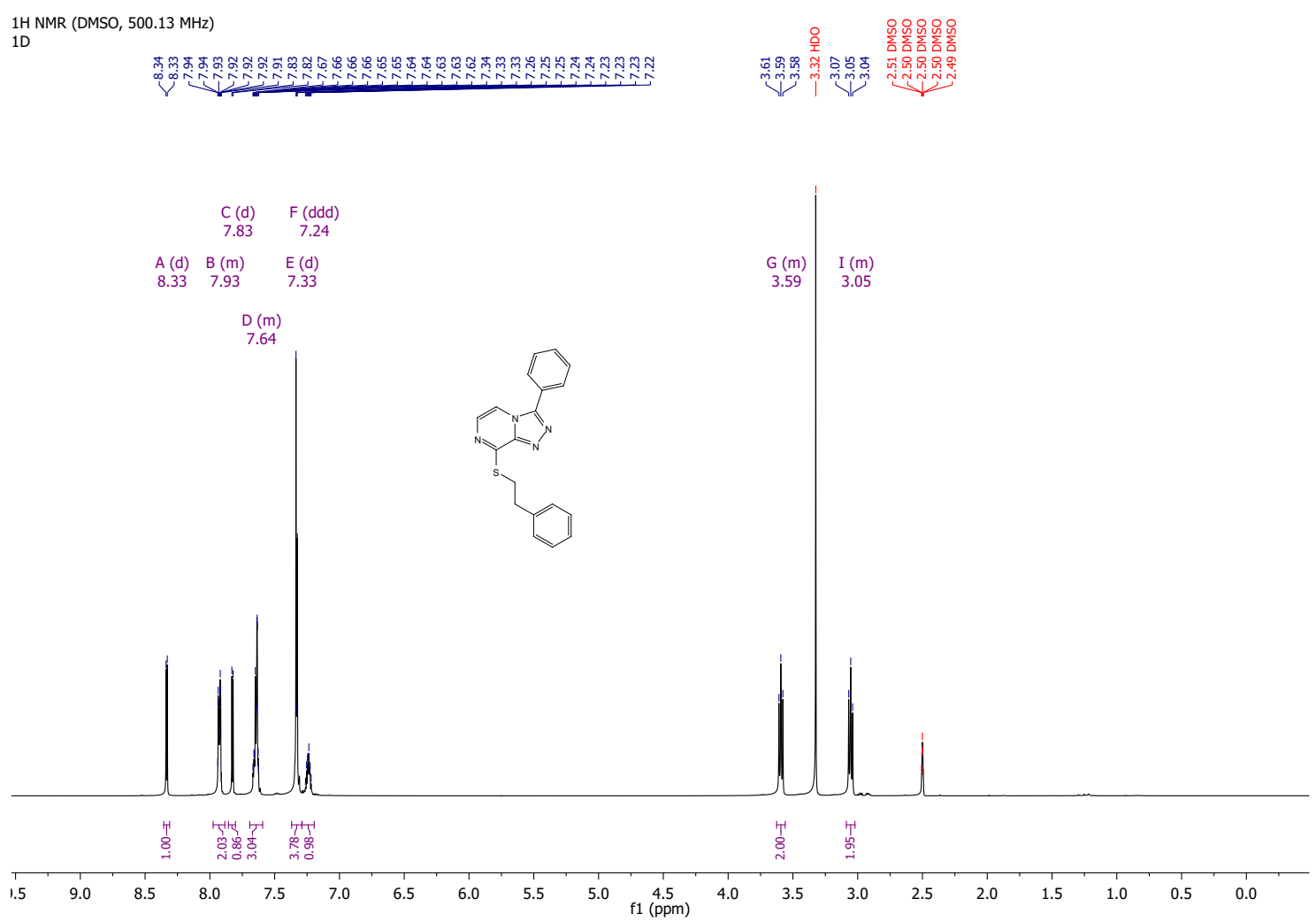

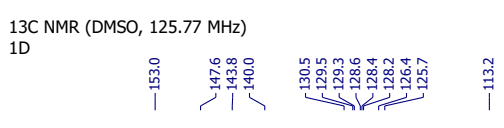

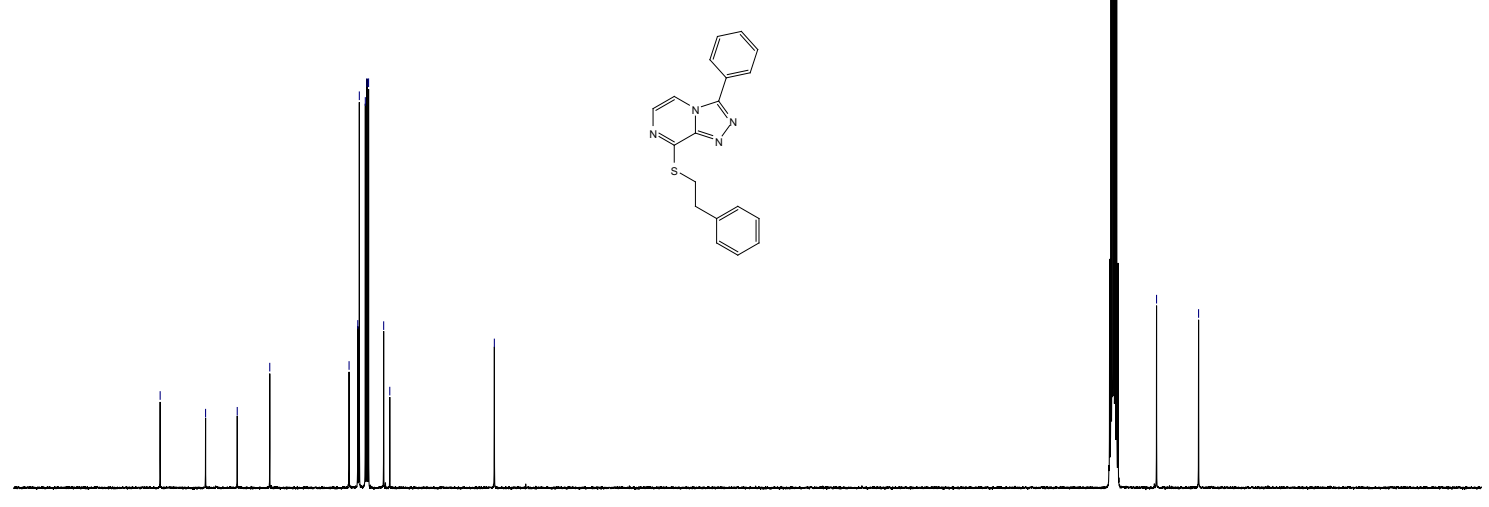

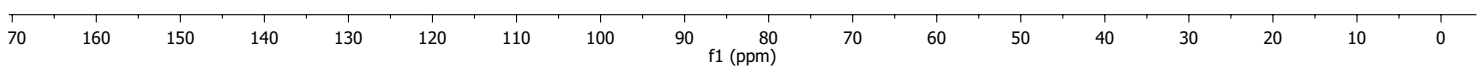


$N$-Phenethyl-3-phenyl-[1,2,4] triazolo[4,3-a]pyrazin-8-amine 33

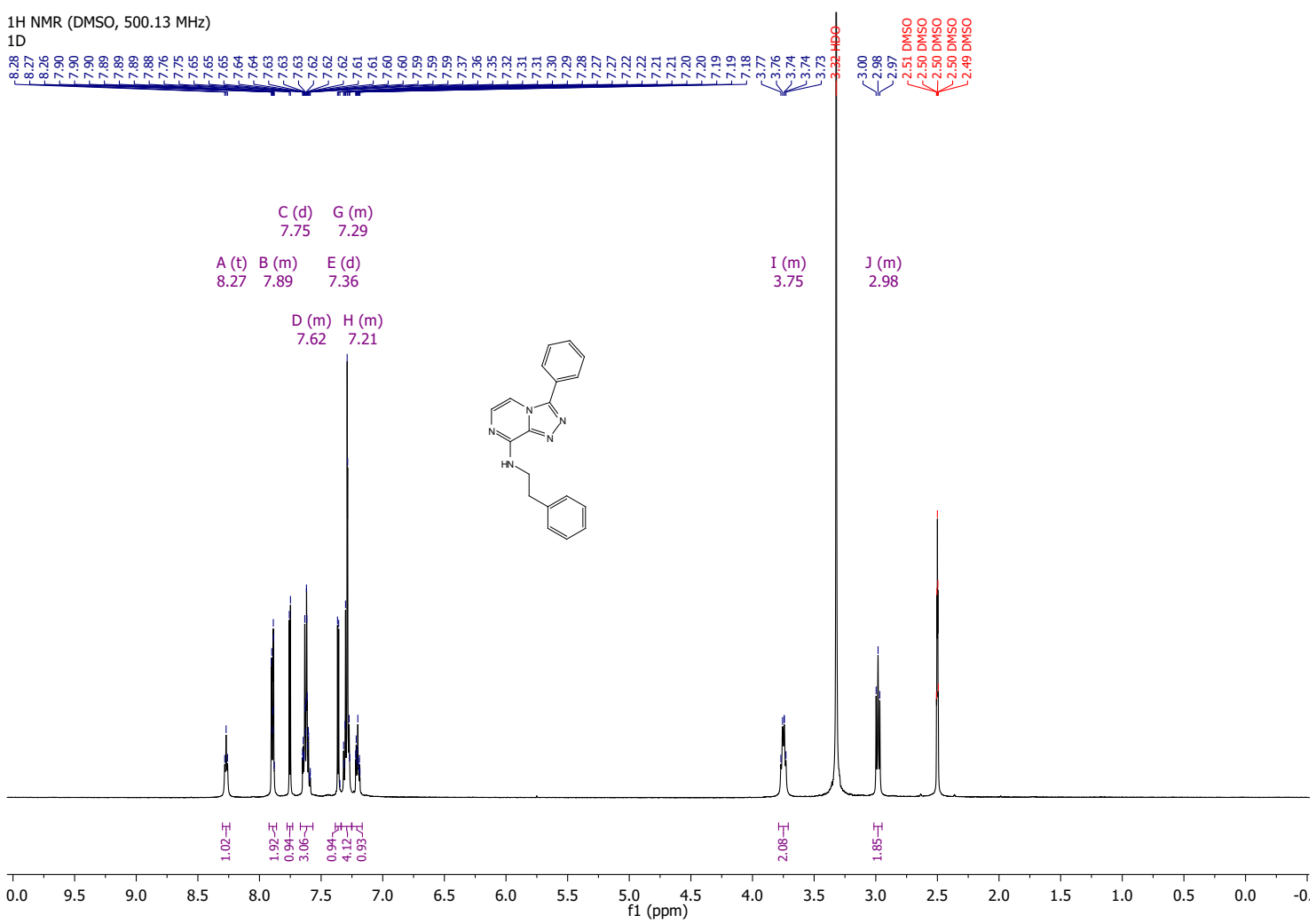

13C NMR (DMSO, $125.77 \mathrm{MHz}$ )

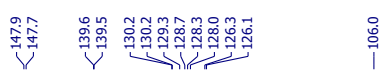
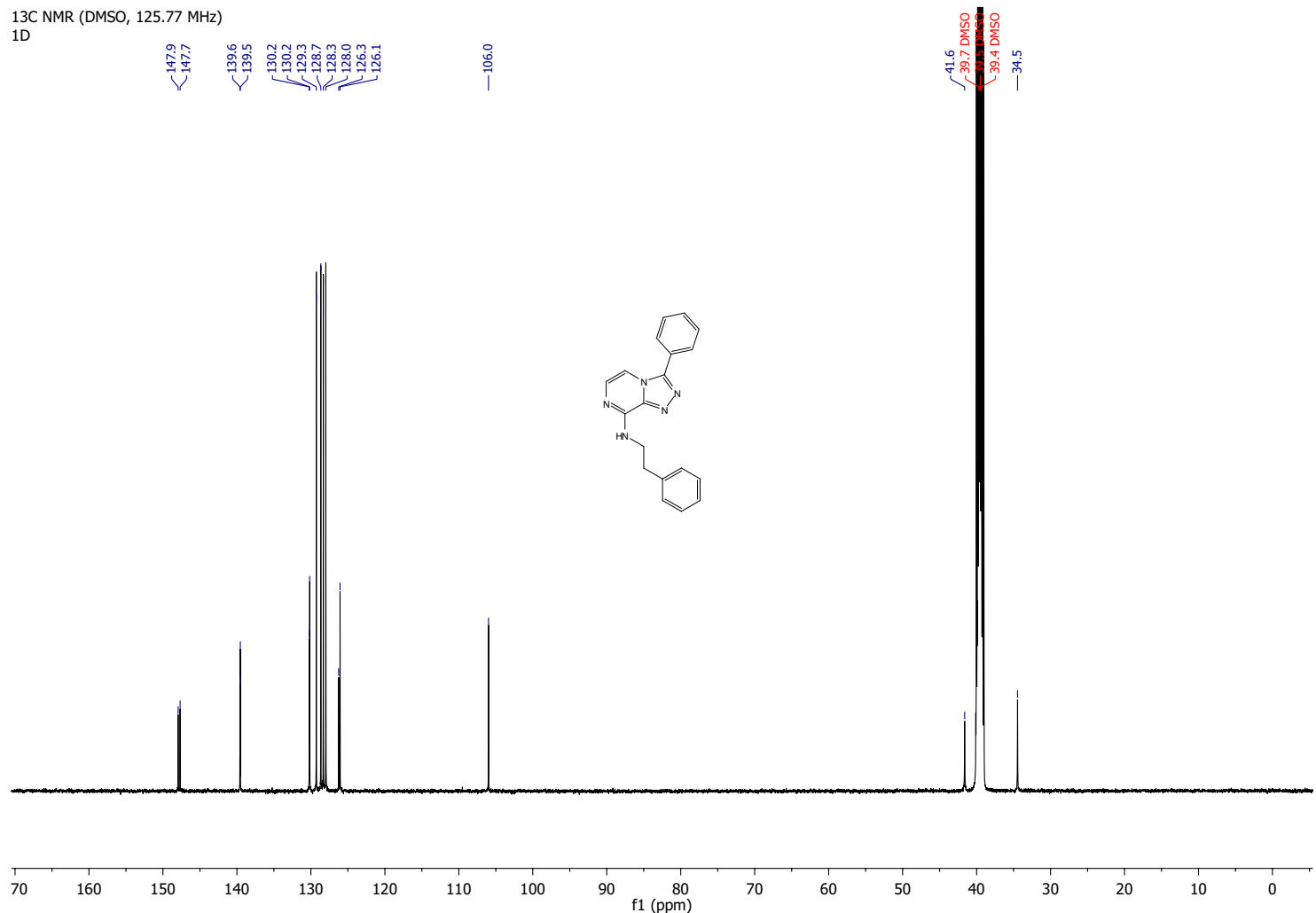
3-(4-Nitrophenyl)-8-phenethoxy-[1,2,4]triazolo[4,3-a]pyrazine 34

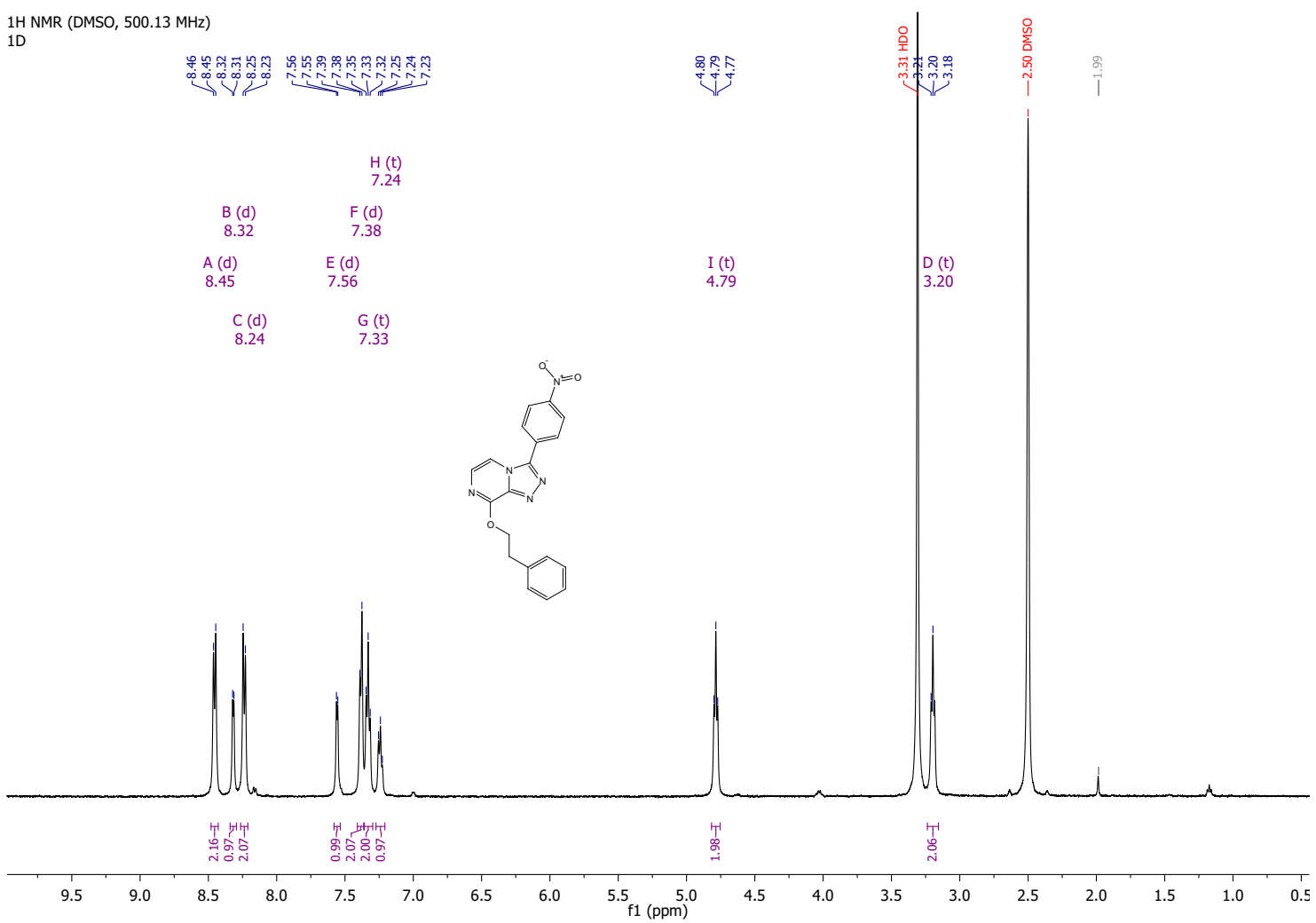

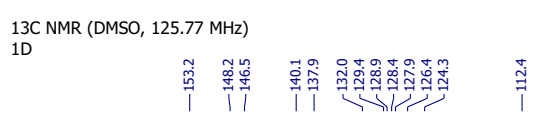
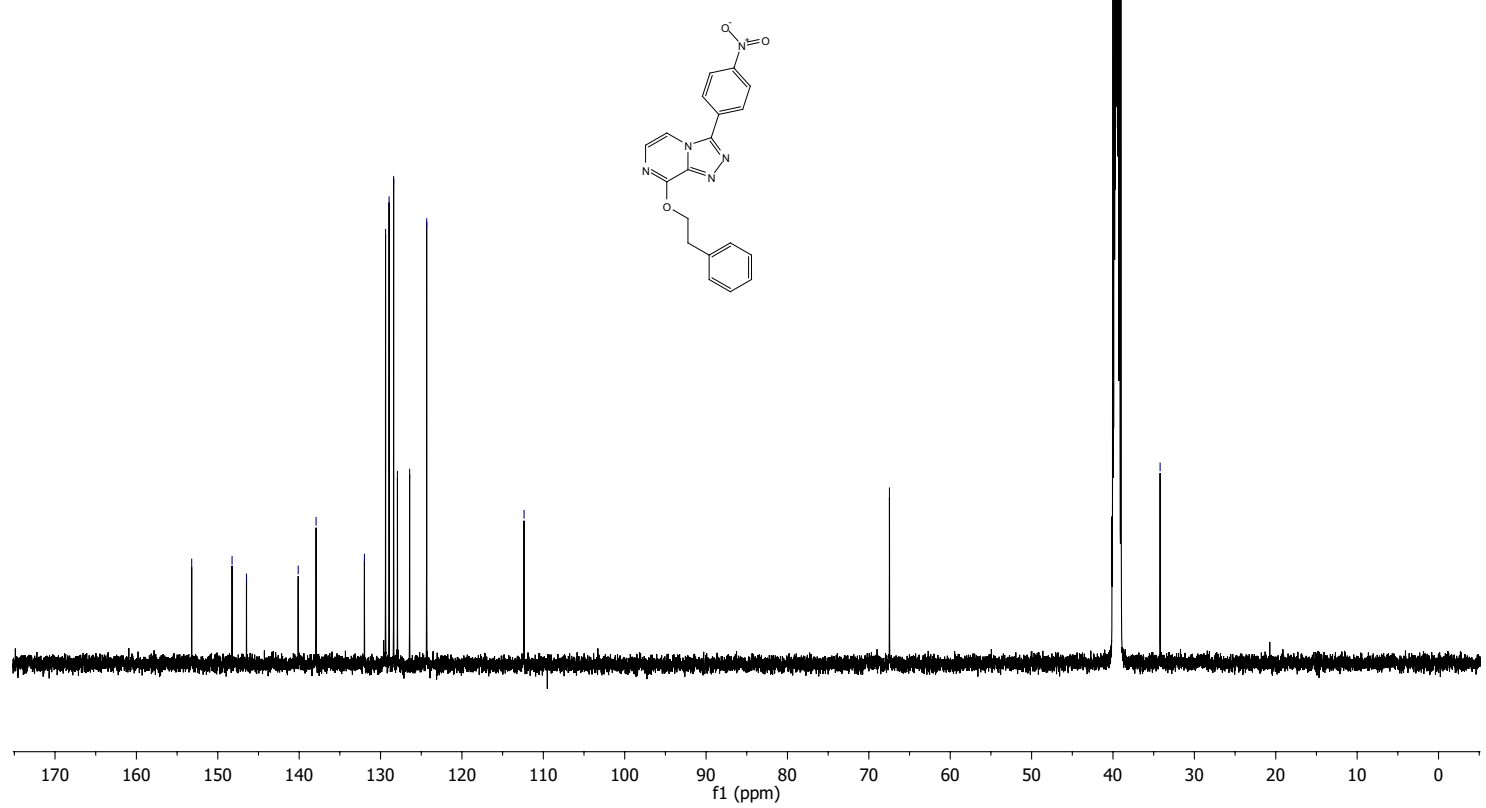
3-(4-Nitrophenyl)-8-(phenethylthio)-[1,2,4] triazolo[4,3-a]pyrazine 35

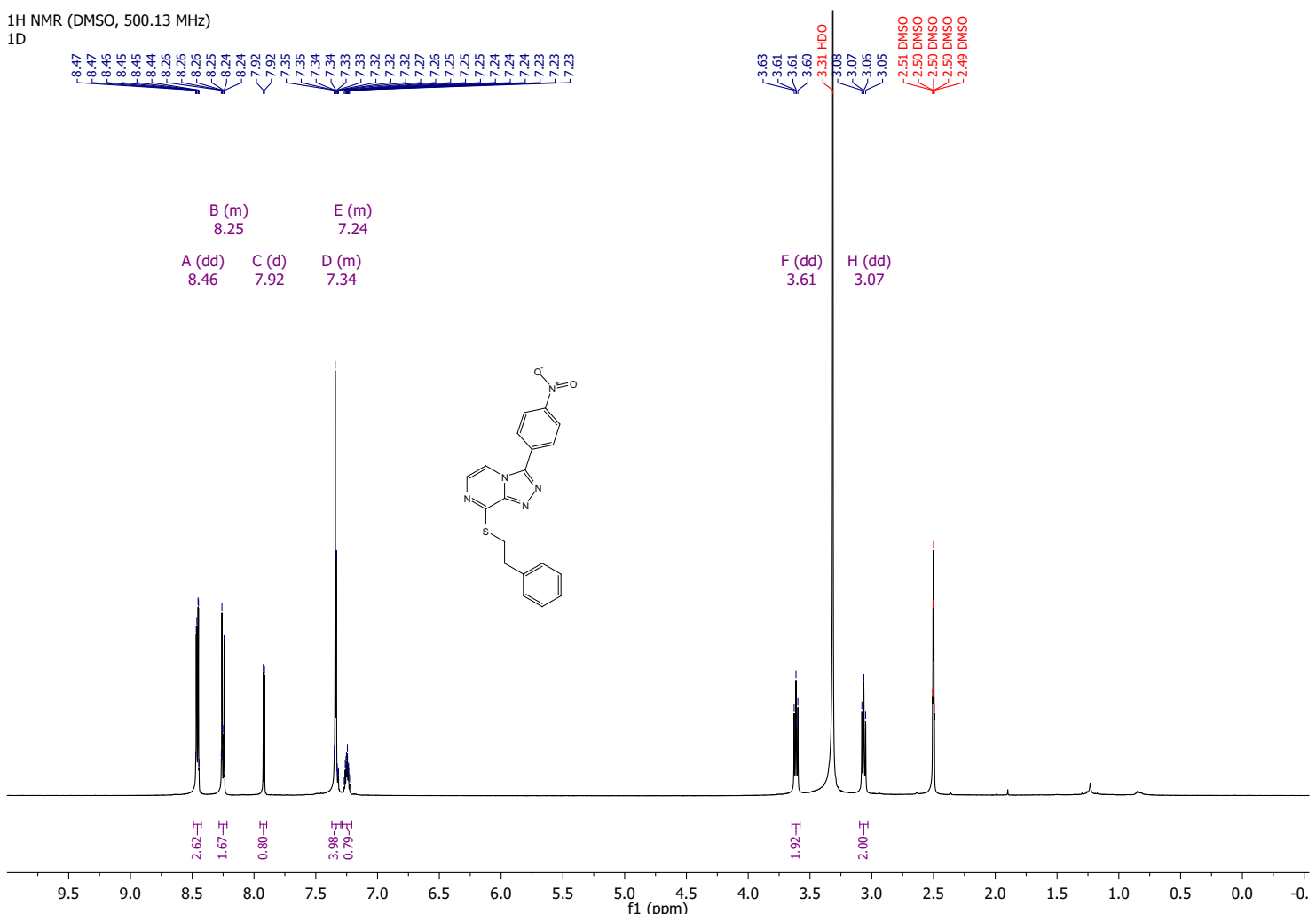

13C NMR (DMSO, $125.77 \mathrm{MHz}$ )

|
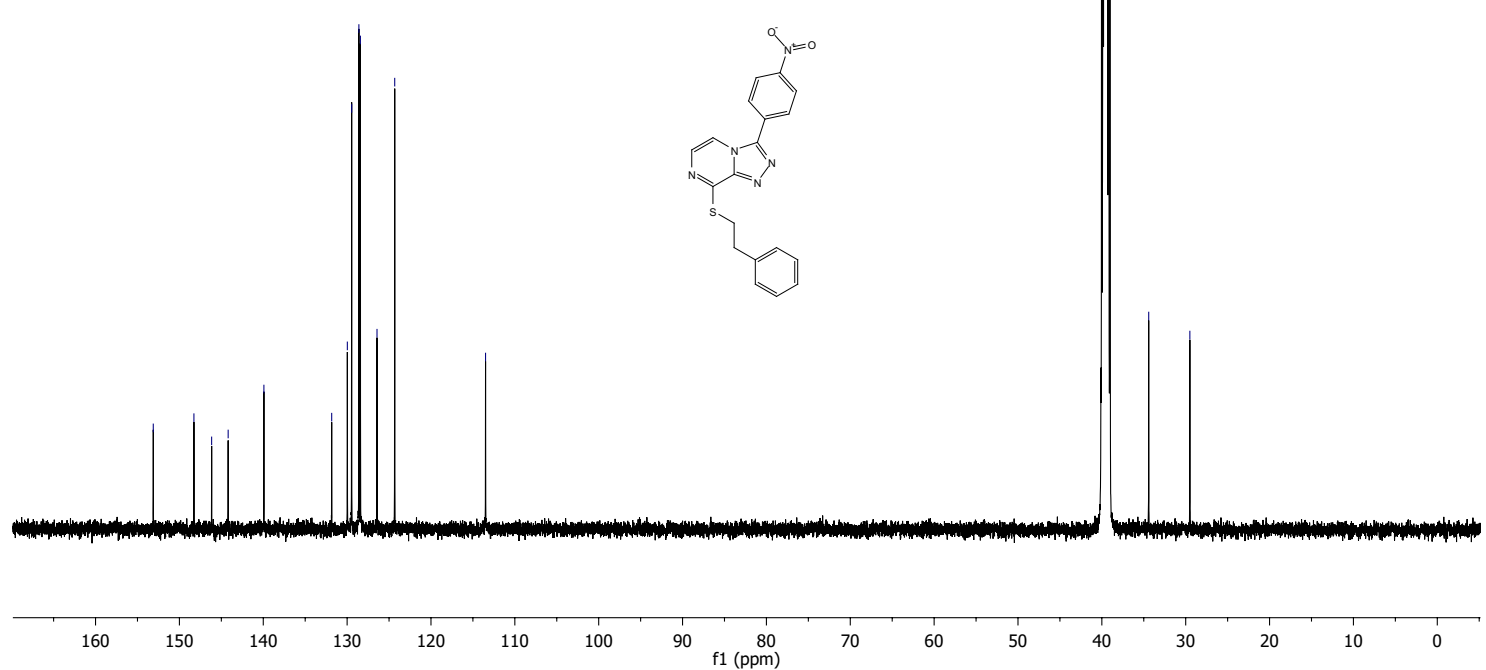
3-(4-Nitrophenyl)- $N$-phenethyl-[1,2,4]triazolo[4,3-a]pyrazin-8-amine 36

1H NMR (DMSO, $500.13 \mathrm{MHz}$ )

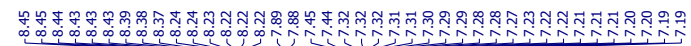
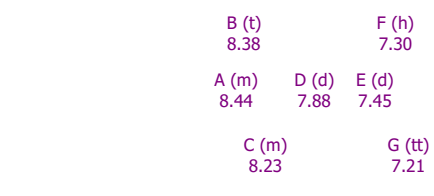

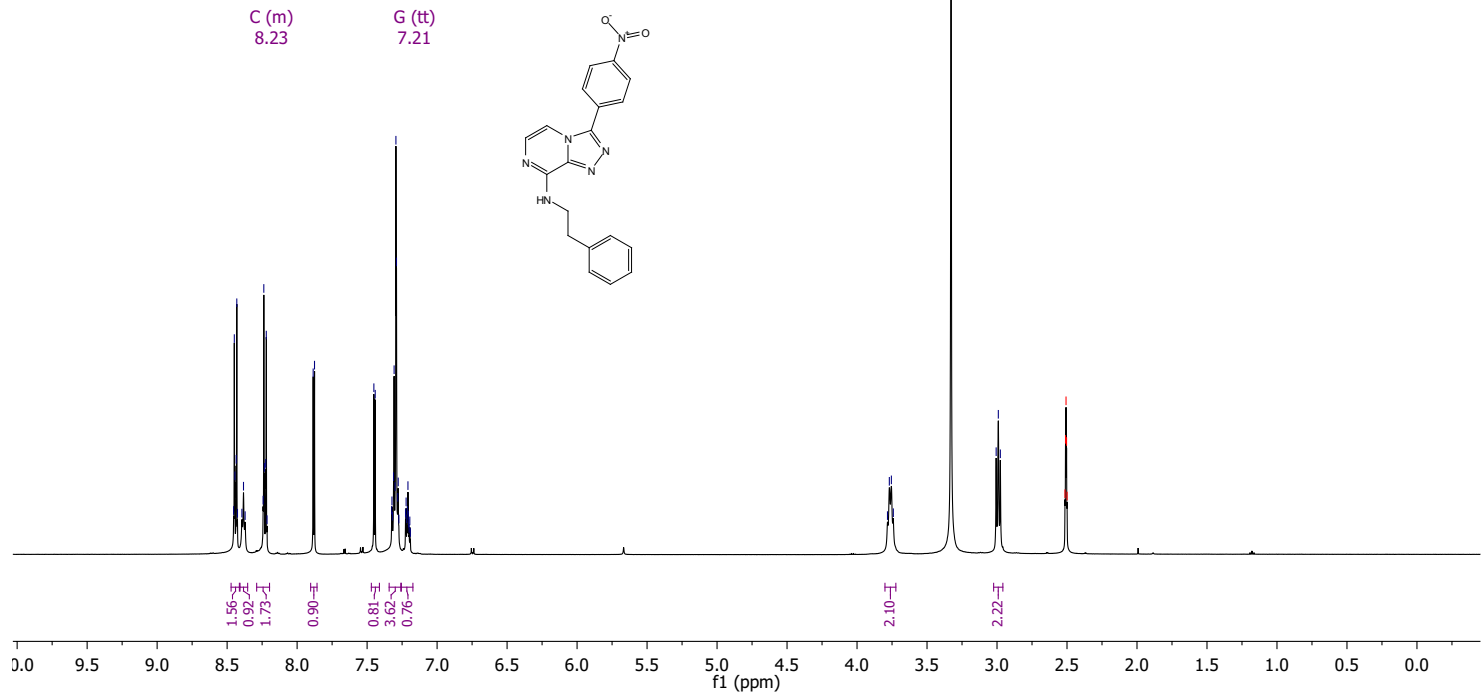

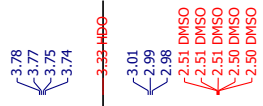

3.7

I (m)
2.99

$13 \mathrm{C}$ NMR (DMSO, $125.77 \mathrm{MHz})$
$1 \mathrm{D}$

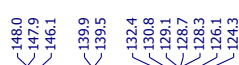

$\stackrel{\text { I }}{\stackrel{0}{0}}$

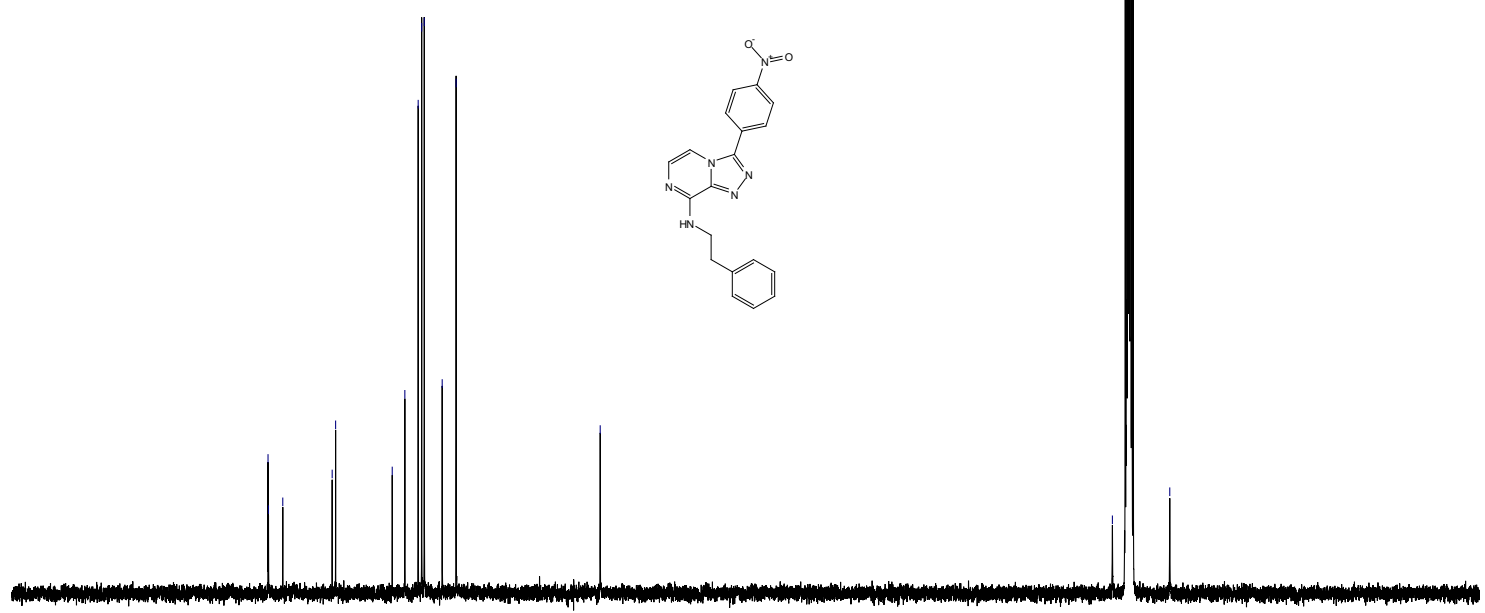

80

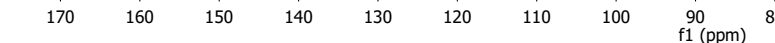


6-Chloro-[1,2,4]triazolo[4,3-a]pyrazine 37

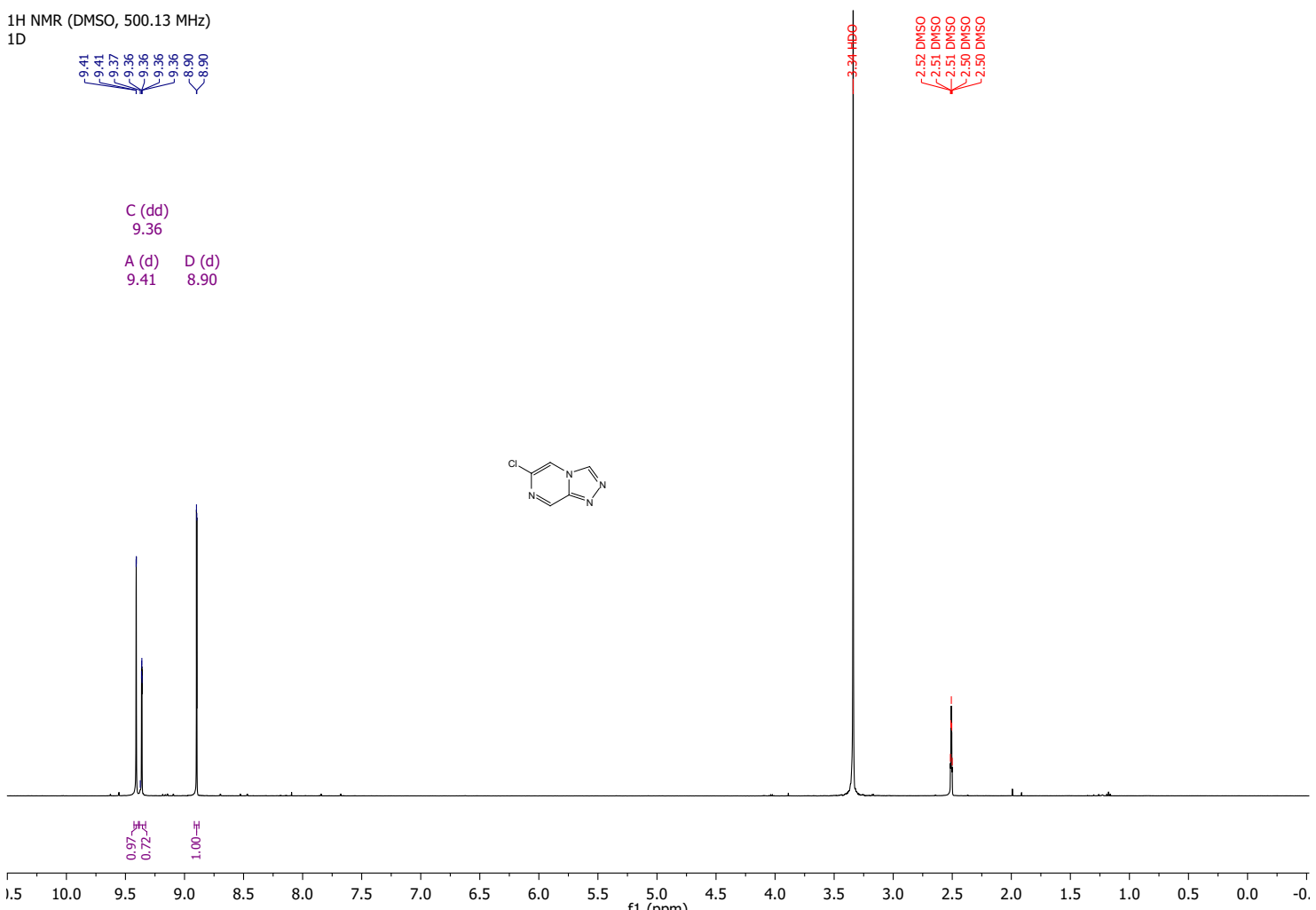

13C NMR (DMSO, $125.77 \mathrm{MHz}$ )

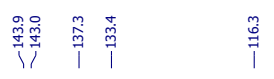
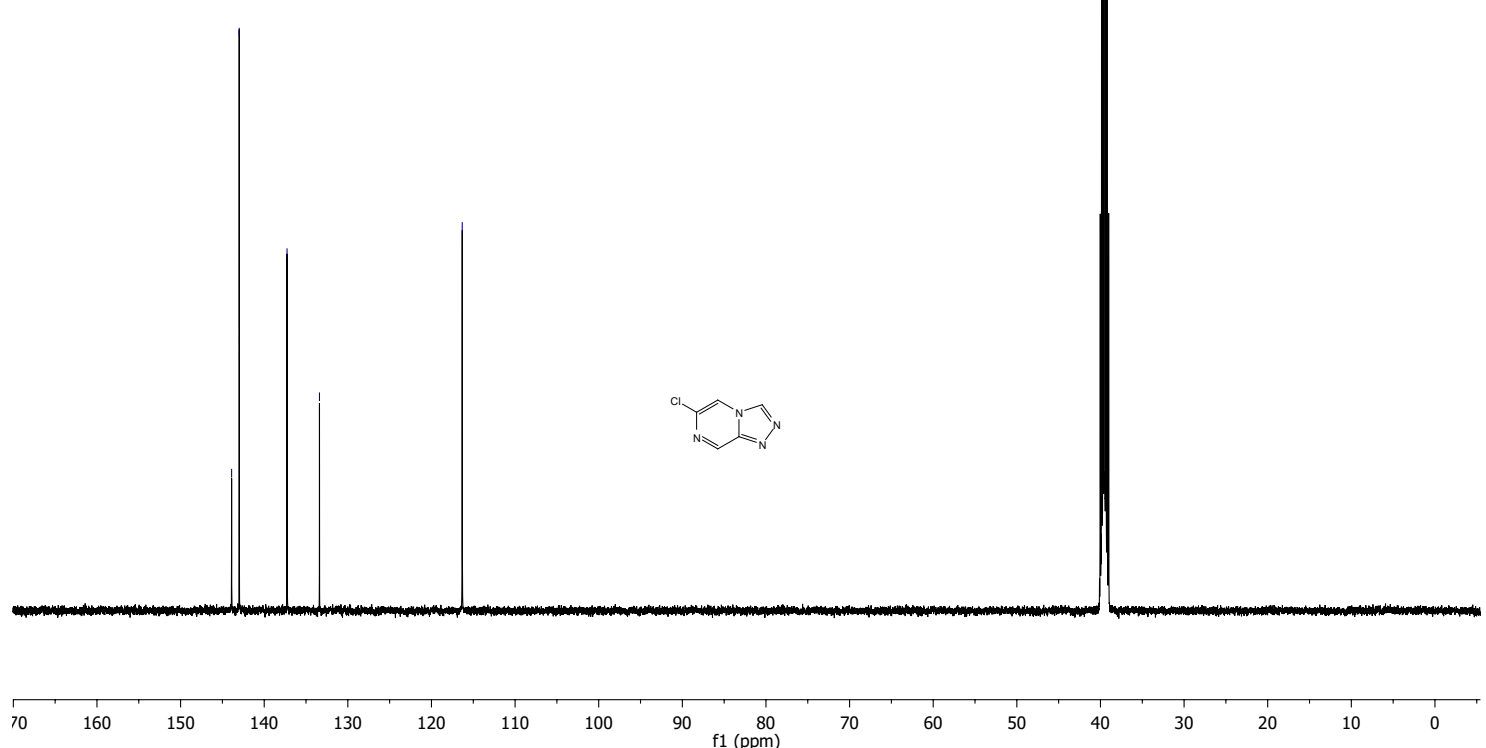
6-Chloro-3-(4-(difluoromethoxy)phenyl)-[1,2,4]triazolo[4,3-a]pyrazine 38

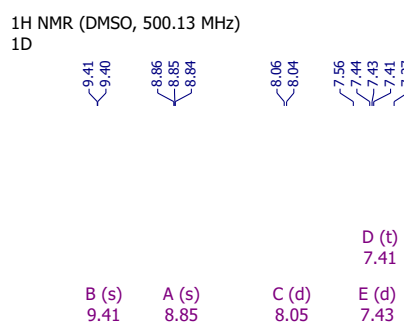

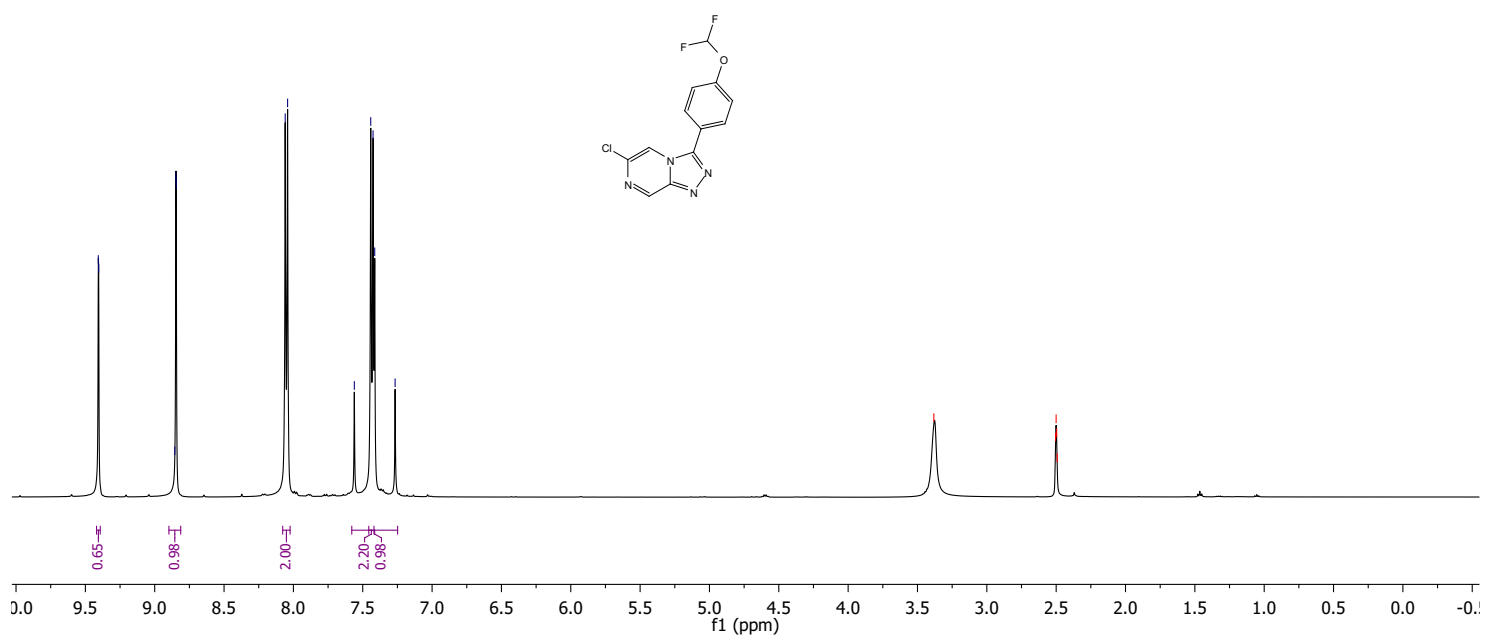

13C NMR (DMSO, 125.77 MHz)

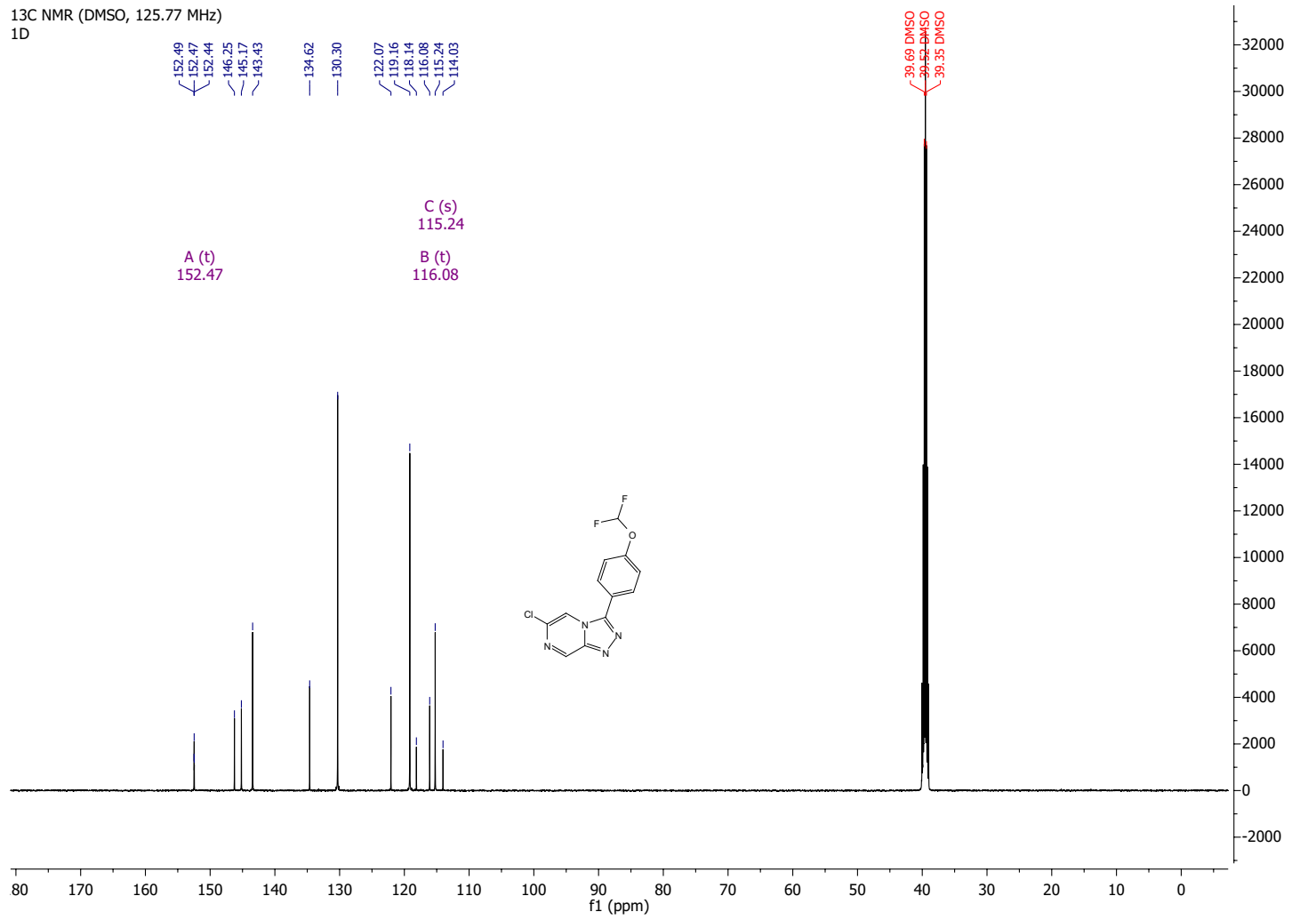




\section{2-Chloro-6-phenethoxypyrazine 42}

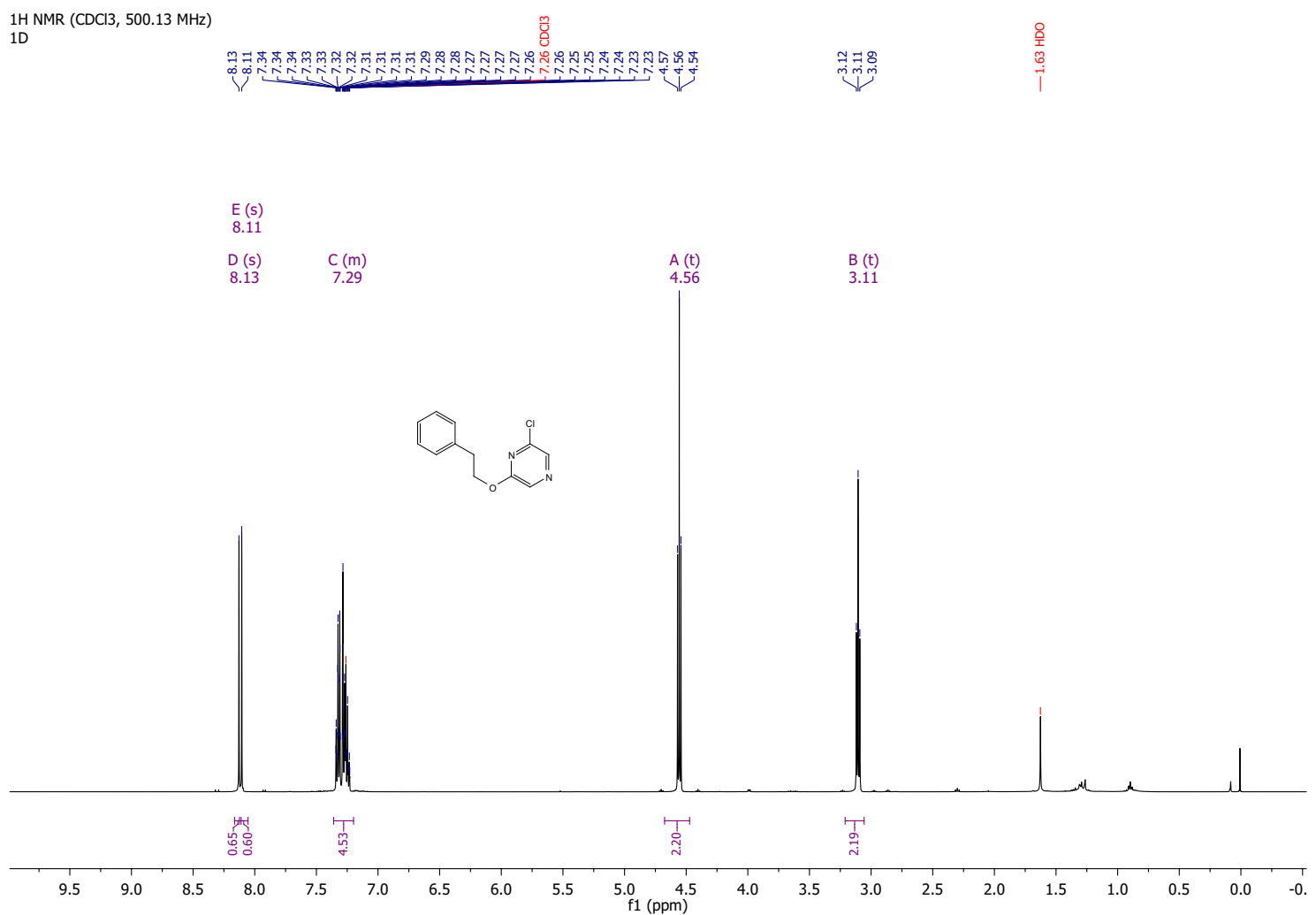

$\begin{array}{lll}13 \mathrm{C} \mathrm{NMR}(\mathrm{CDCl} 3,125.77 \mathrm{MHz}) & \\ 1 \mathrm{D} & & \end{array}$

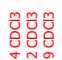

点

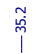

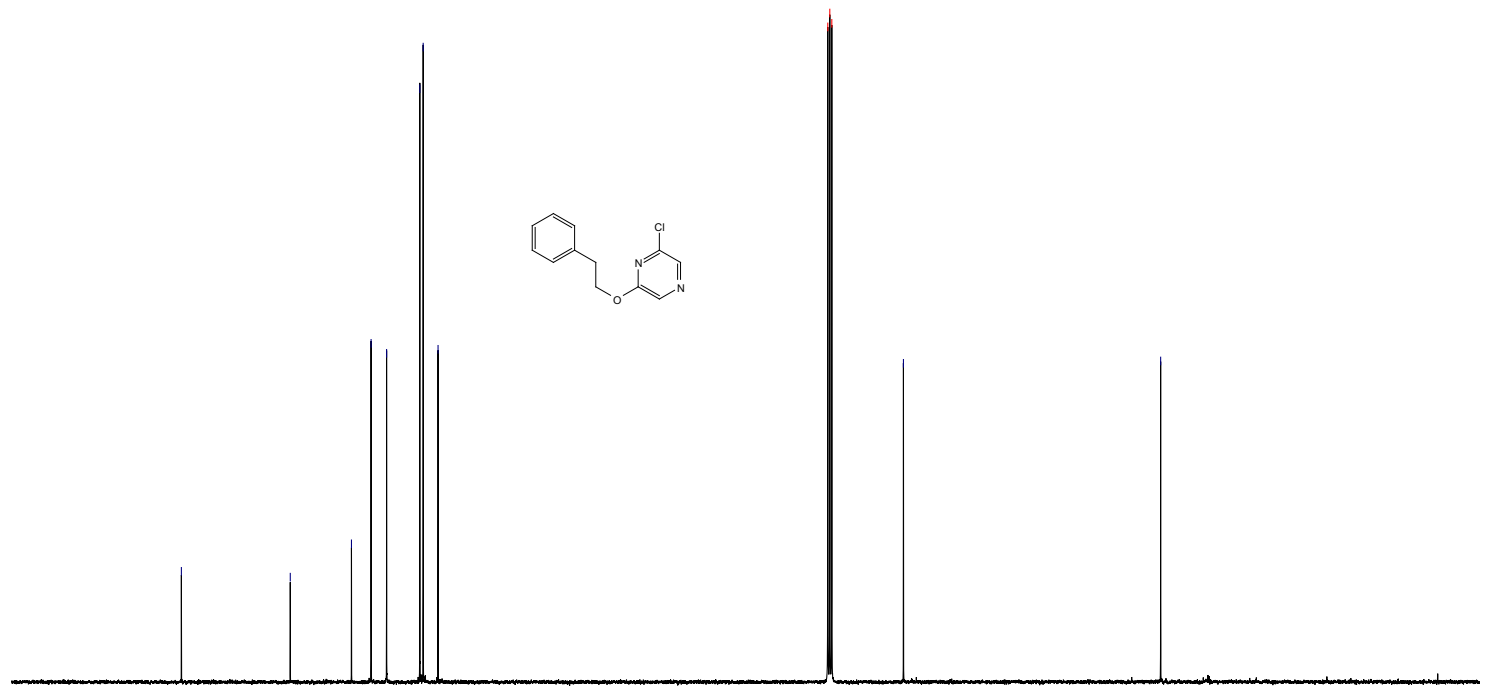

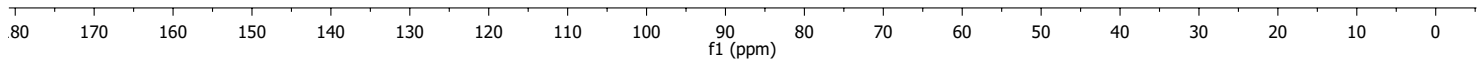




\section{2-Bromo-6-phenethoxypyrazine 43}
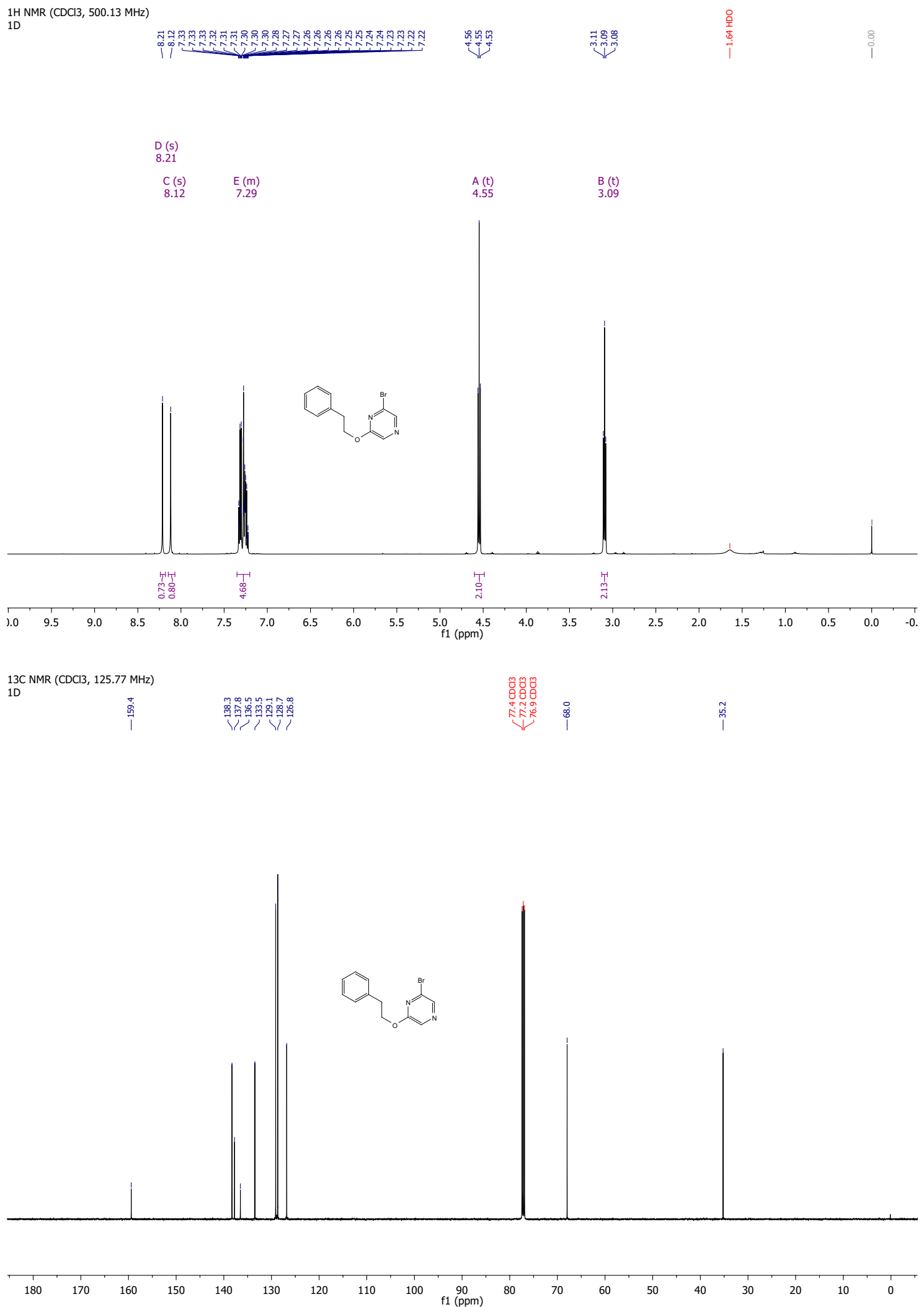


\section{2-Iodo-6-phenethoxypyrazine 44}

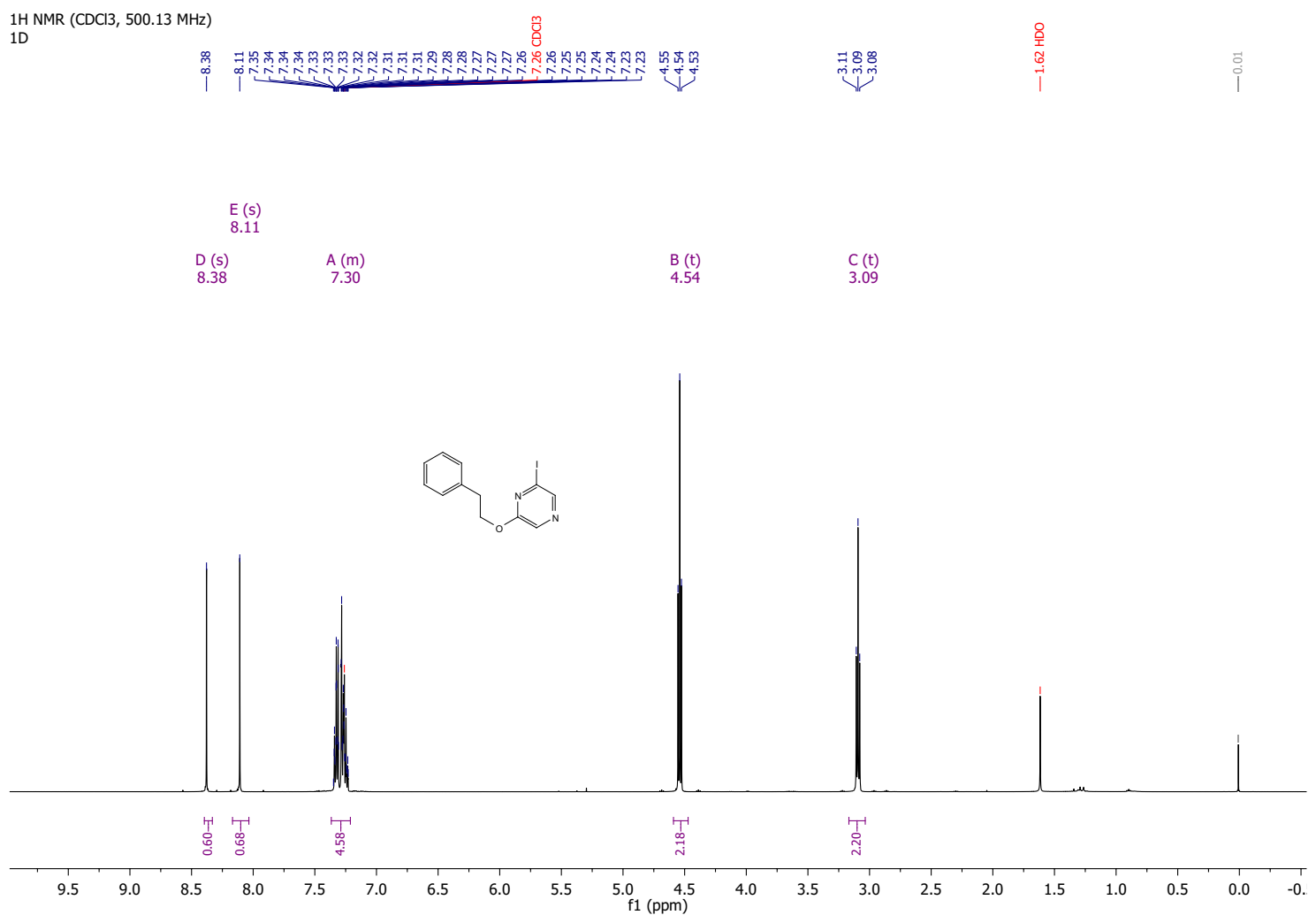

13C NMR (CDCl3, 125.77 MHz)
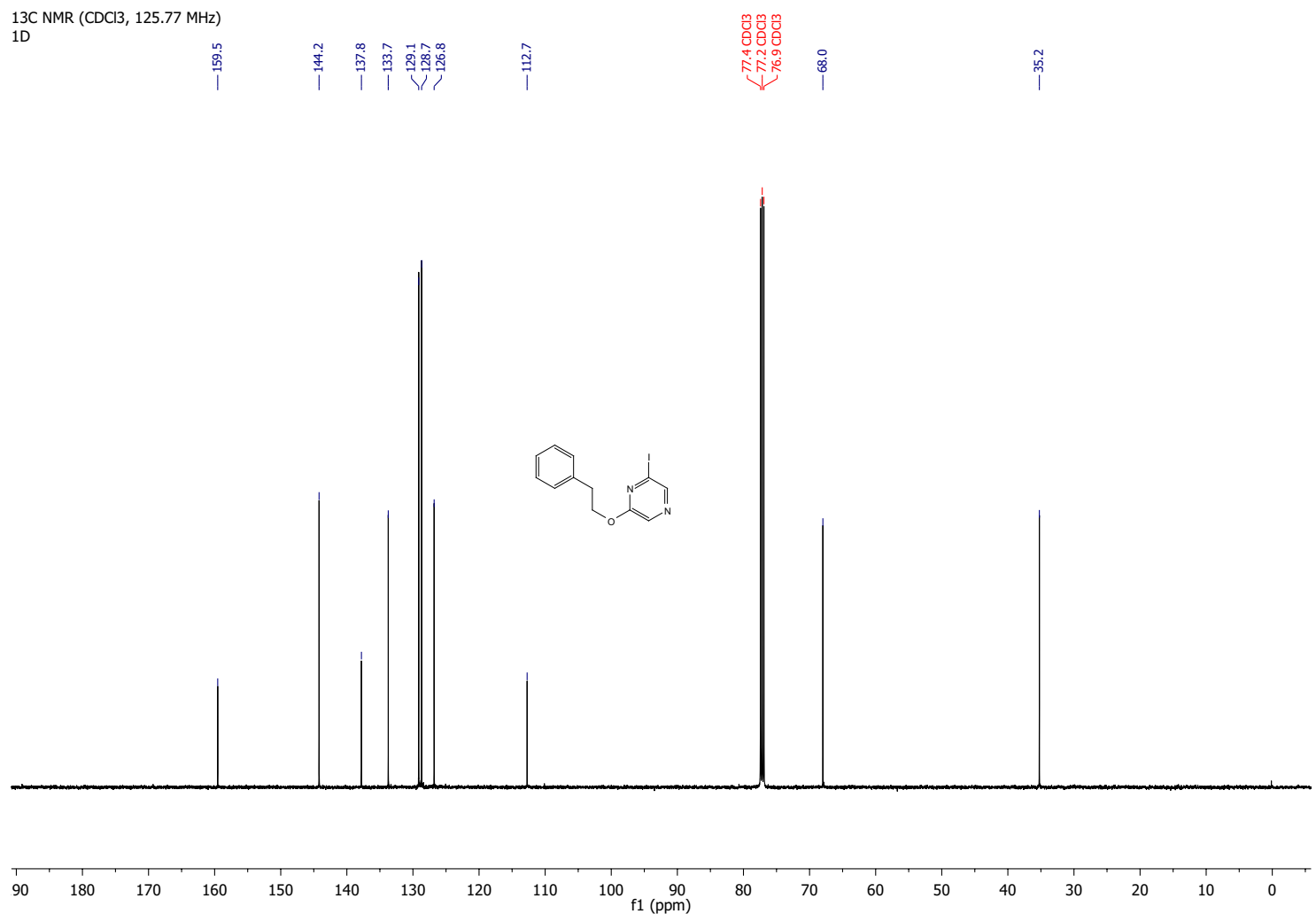
5-Chloro-[1,2,4] triazolo[4,3-a]pyrazine $45 \mathrm{a}$

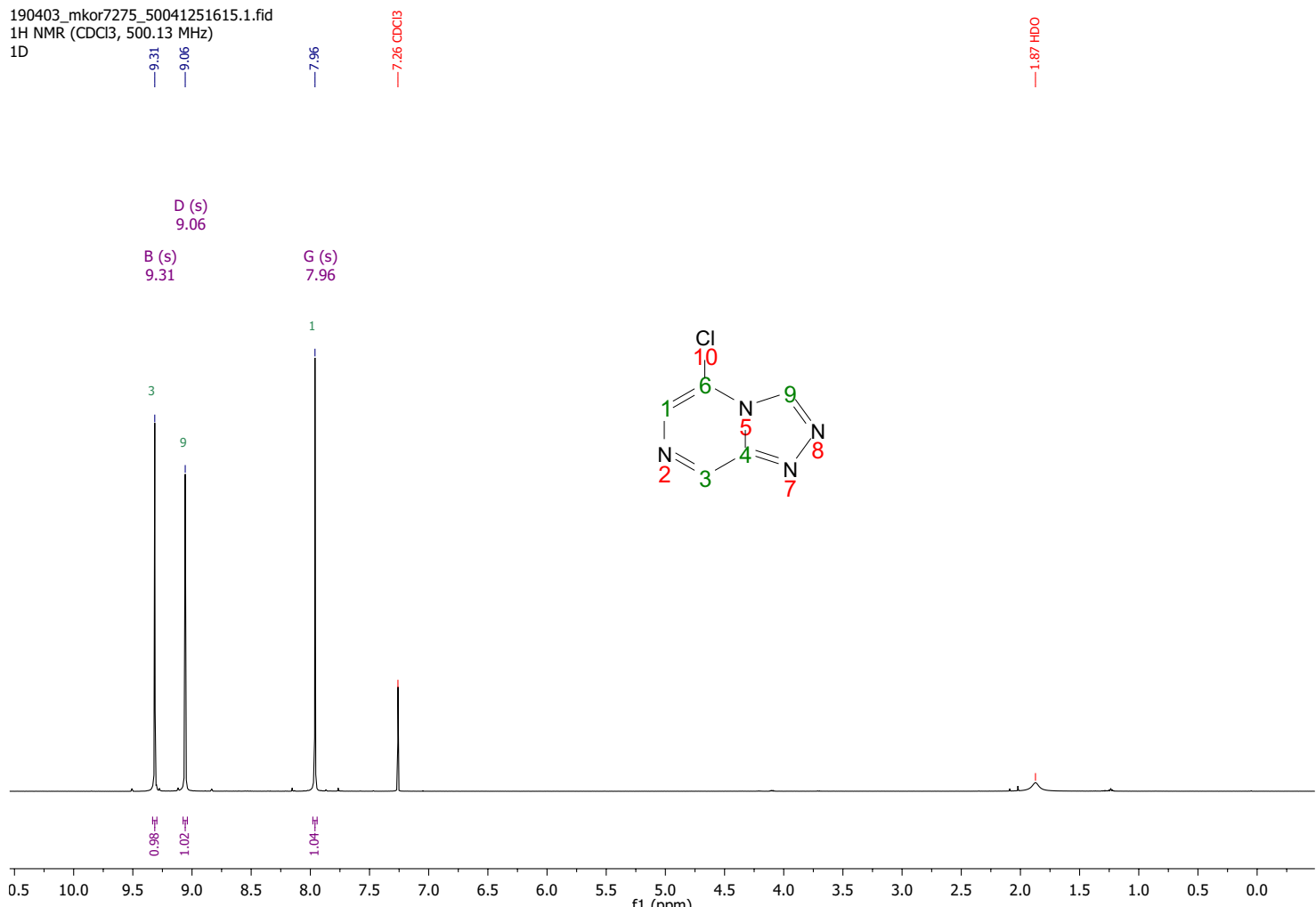

190403_mkor7275_50041251615.4.fid

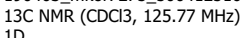

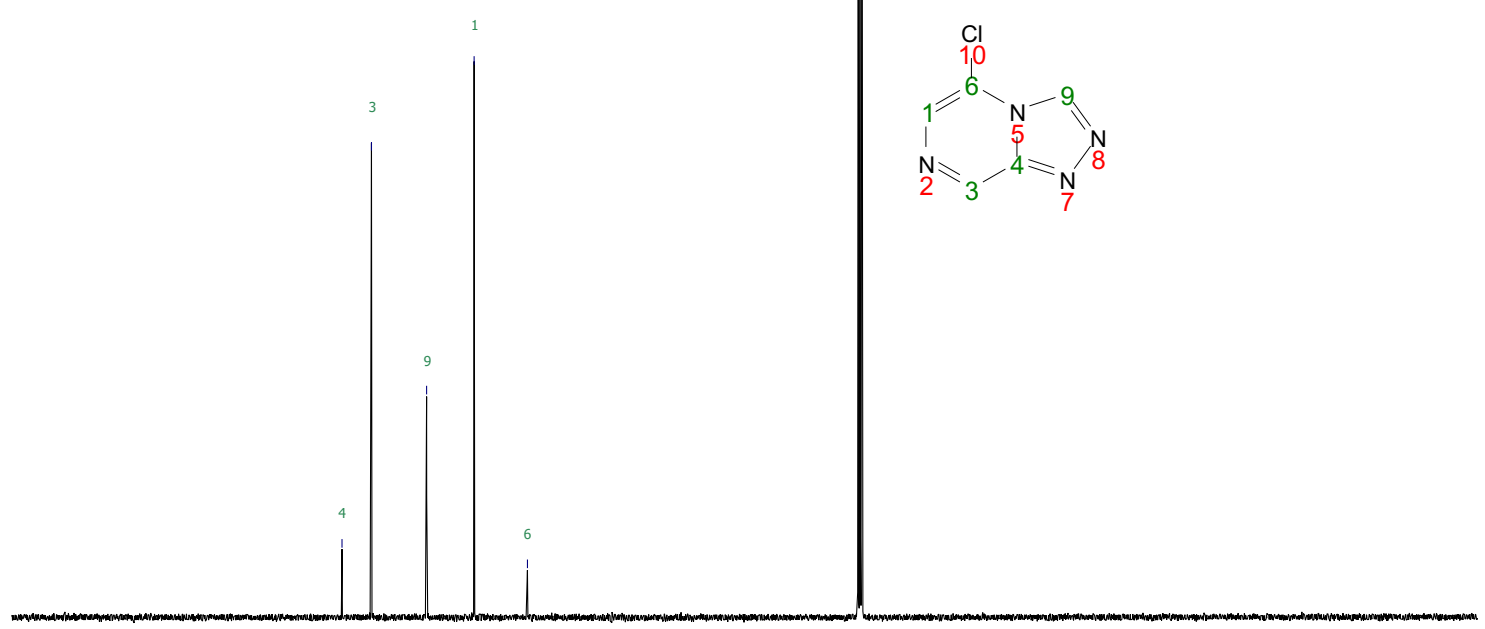

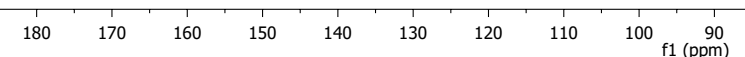



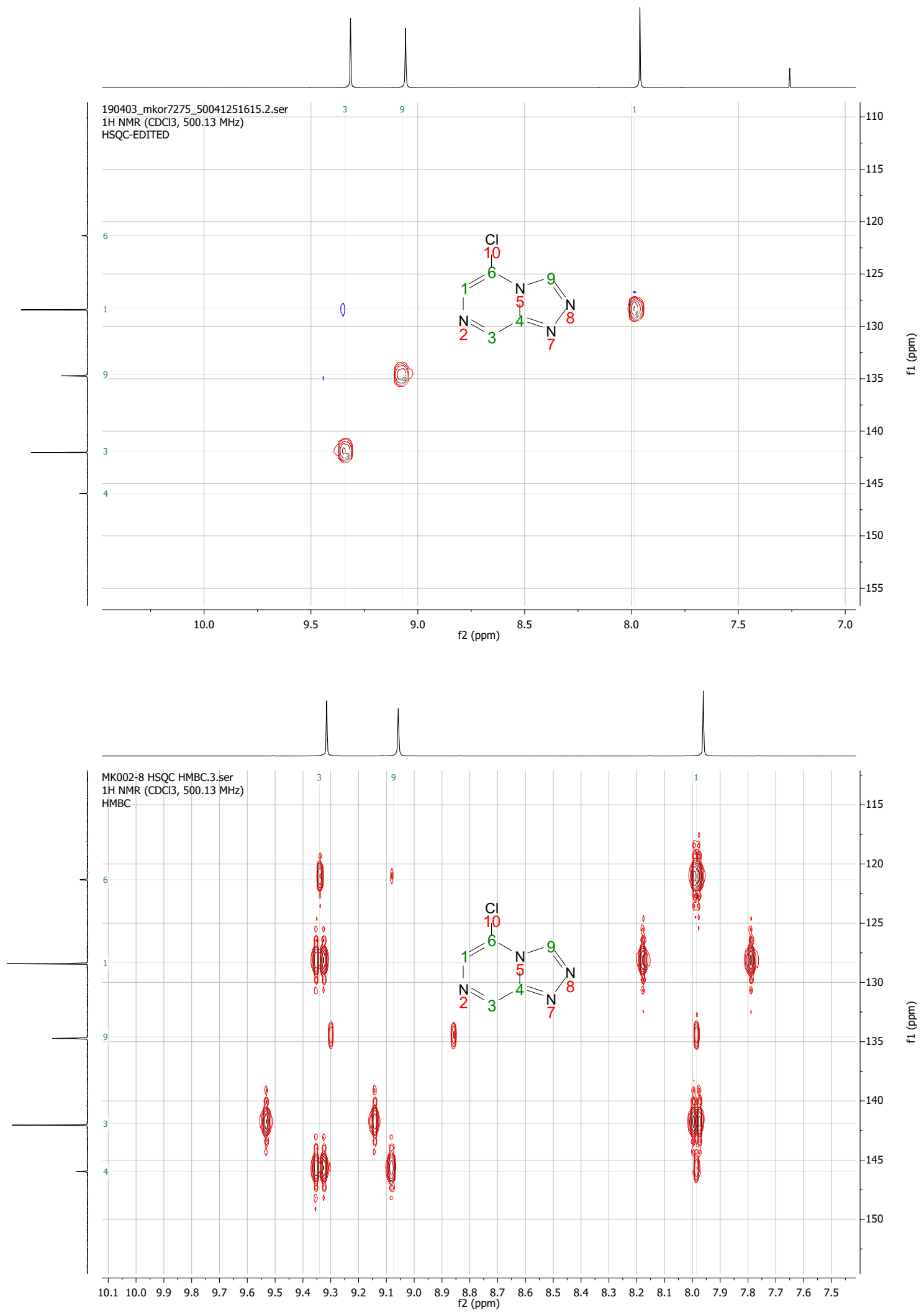
5-Chloro-3-(4-methoxyphenyl)-[1,2,4] triazolo[4,3- $a]$ pyrazine 45b
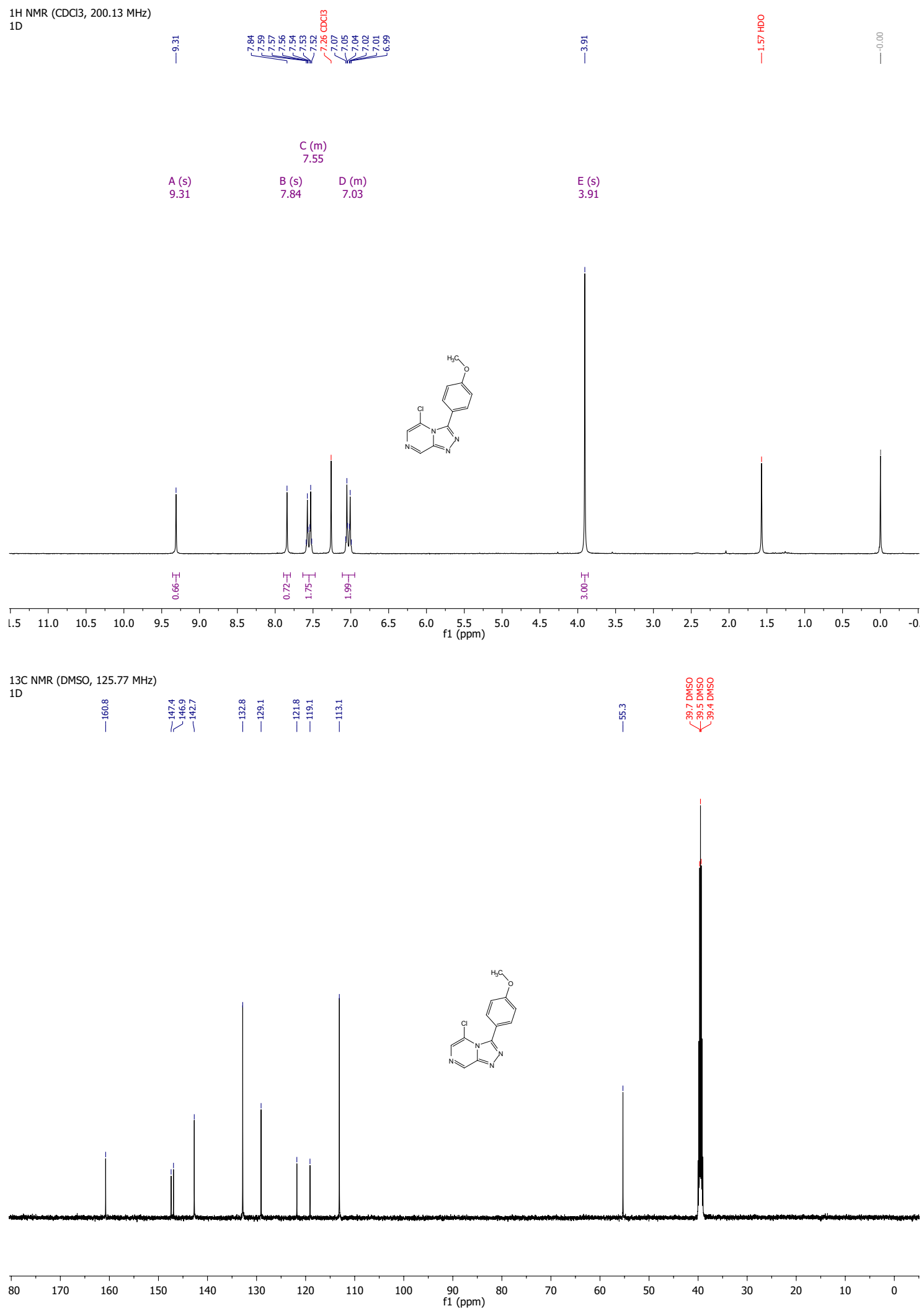
5-Chloro-3-(4-nitrophenyl)-[1,2,4]triazolo[4,3-a]pyrazine 45c
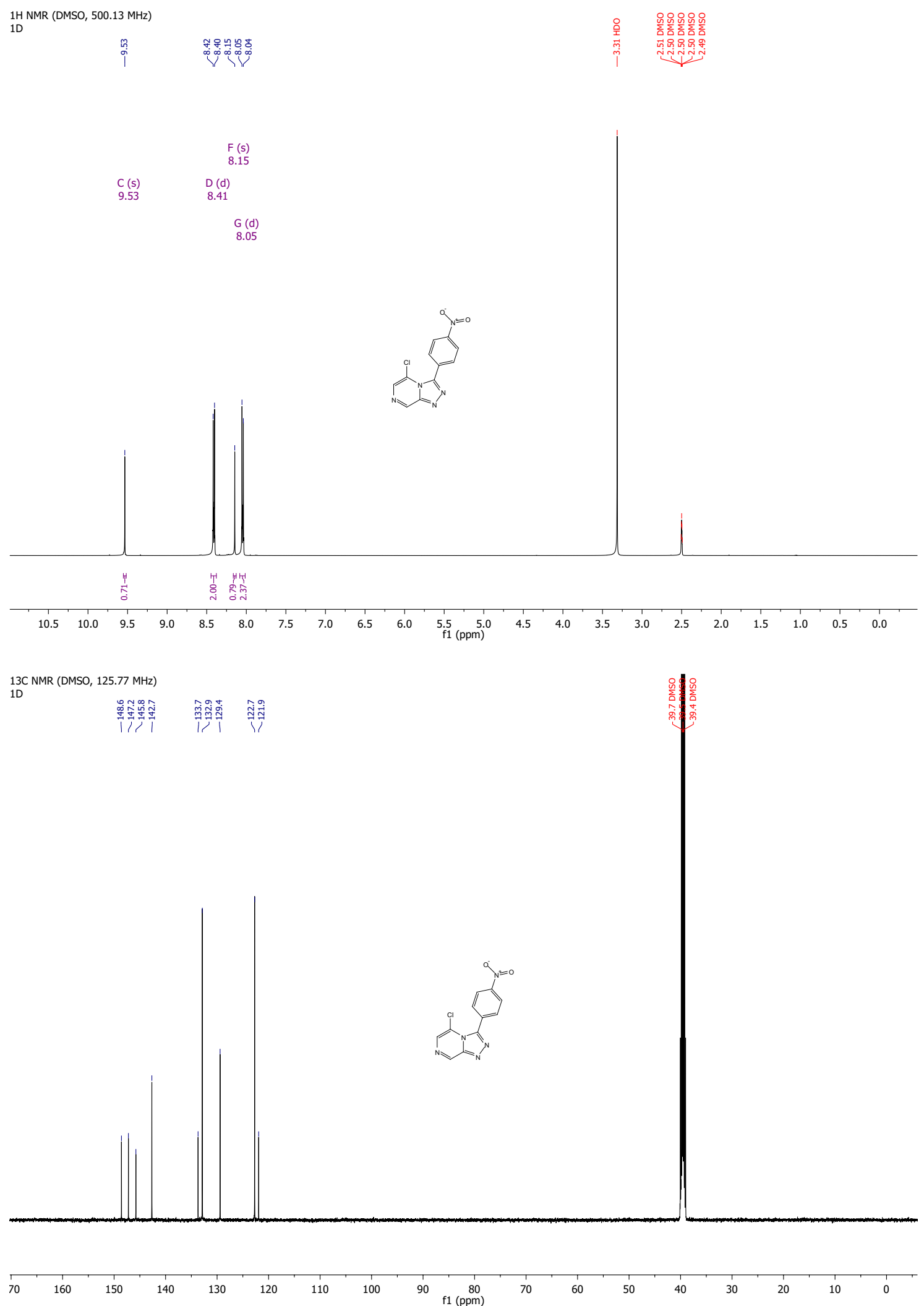
5-Chloro-3-(2-methoxyphenyl)-[1,2,4] triazolo[4,3-a]pyrazine 45d
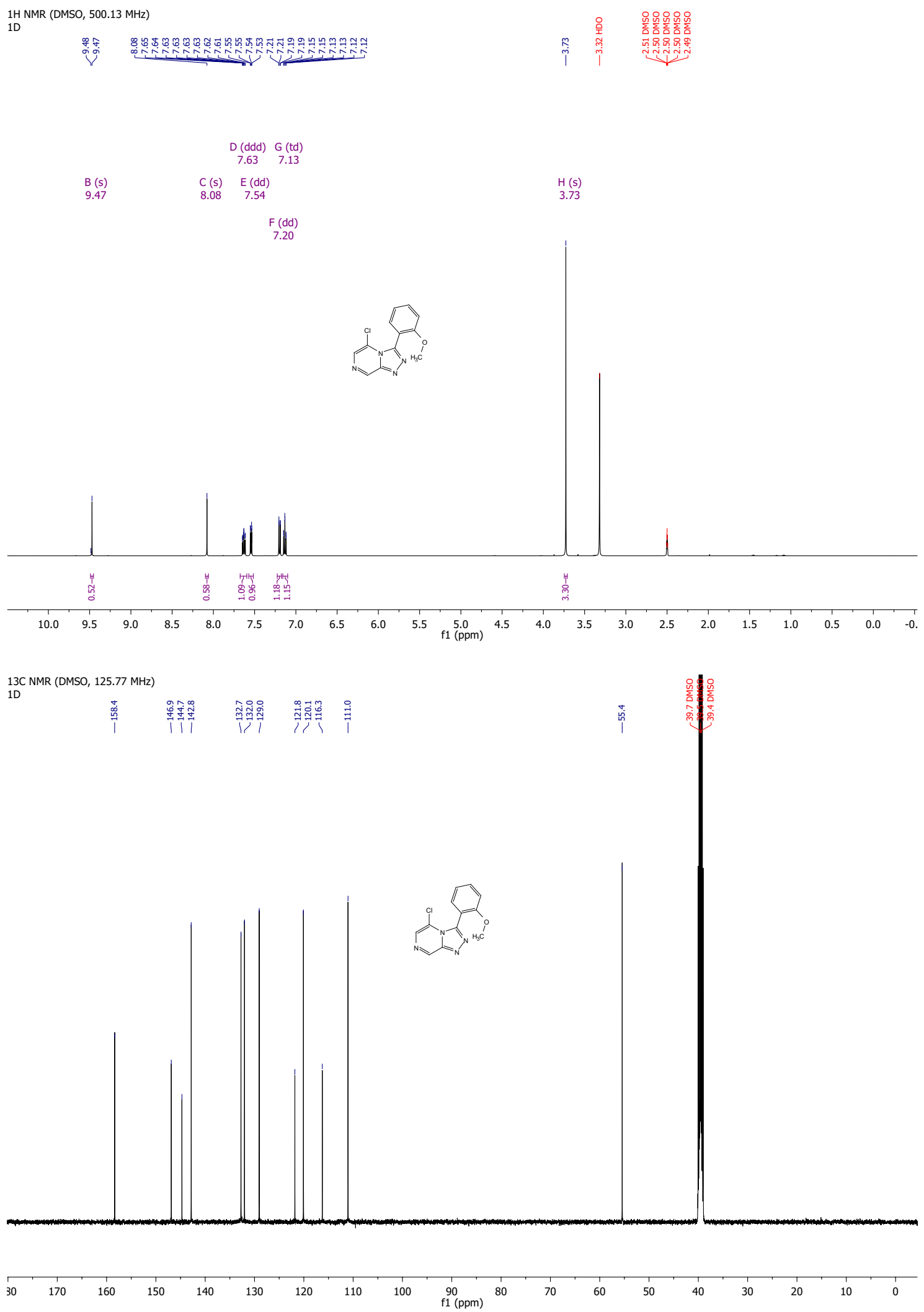
5-Chloro-3-(2-nitrophenyl)-[1,2,4] triazolo[4,3-a]pyrazine 45e

190710_mkor7275_50041302252.1.fid $1 \mathrm{H}$ NMR (DMSO, $500.13 \mathrm{MHz}$ )

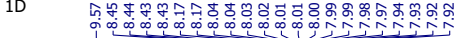

8.17

\begin{tabular}{ccc} 
& \multicolumn{2}{c}{$E(s)$} \\
& 8.17 \\
B (s) & $C(d d)$ & G (dd) \\
9.57 & 8.44 & 7.93 \\
& & \\
& & $F(m)$ \\
& & 8.01
\end{tabular}

$\mathrm{F}(\mathrm{m})$
8.01

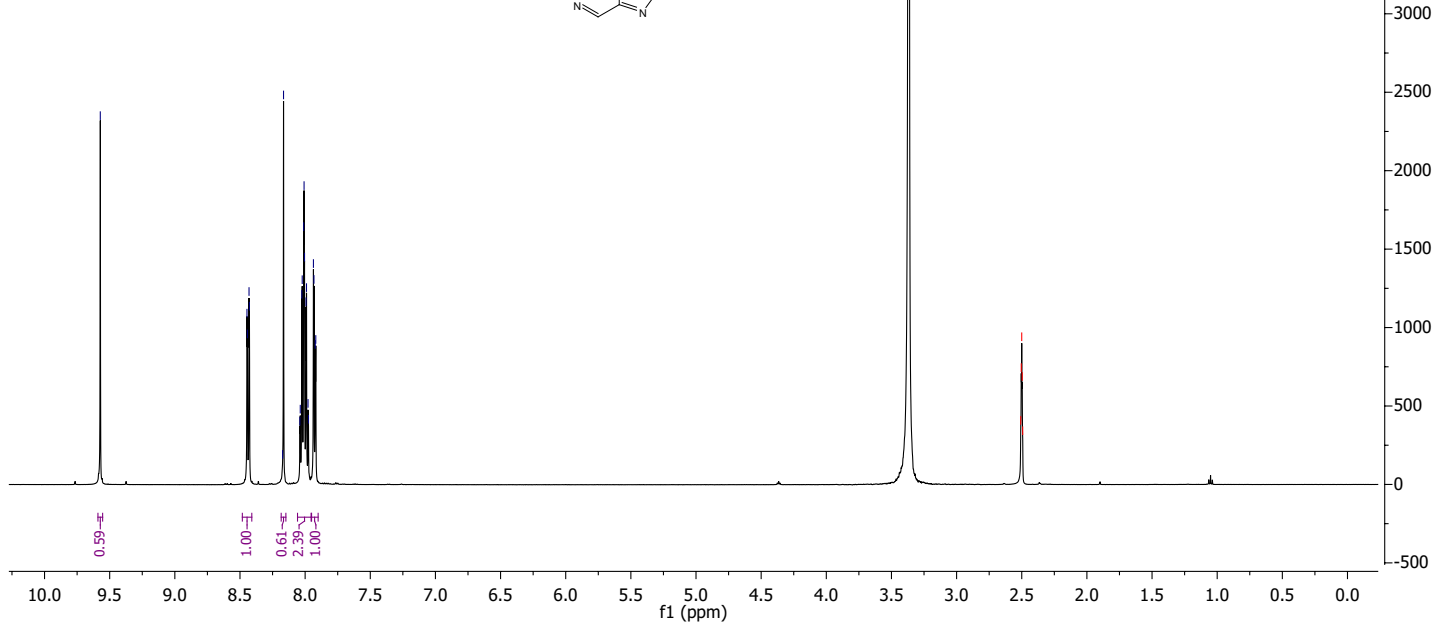

13C NMR (DMSO, $125.77 \mathrm{MHz}$ )

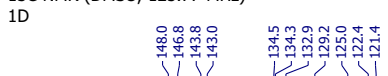

雪;

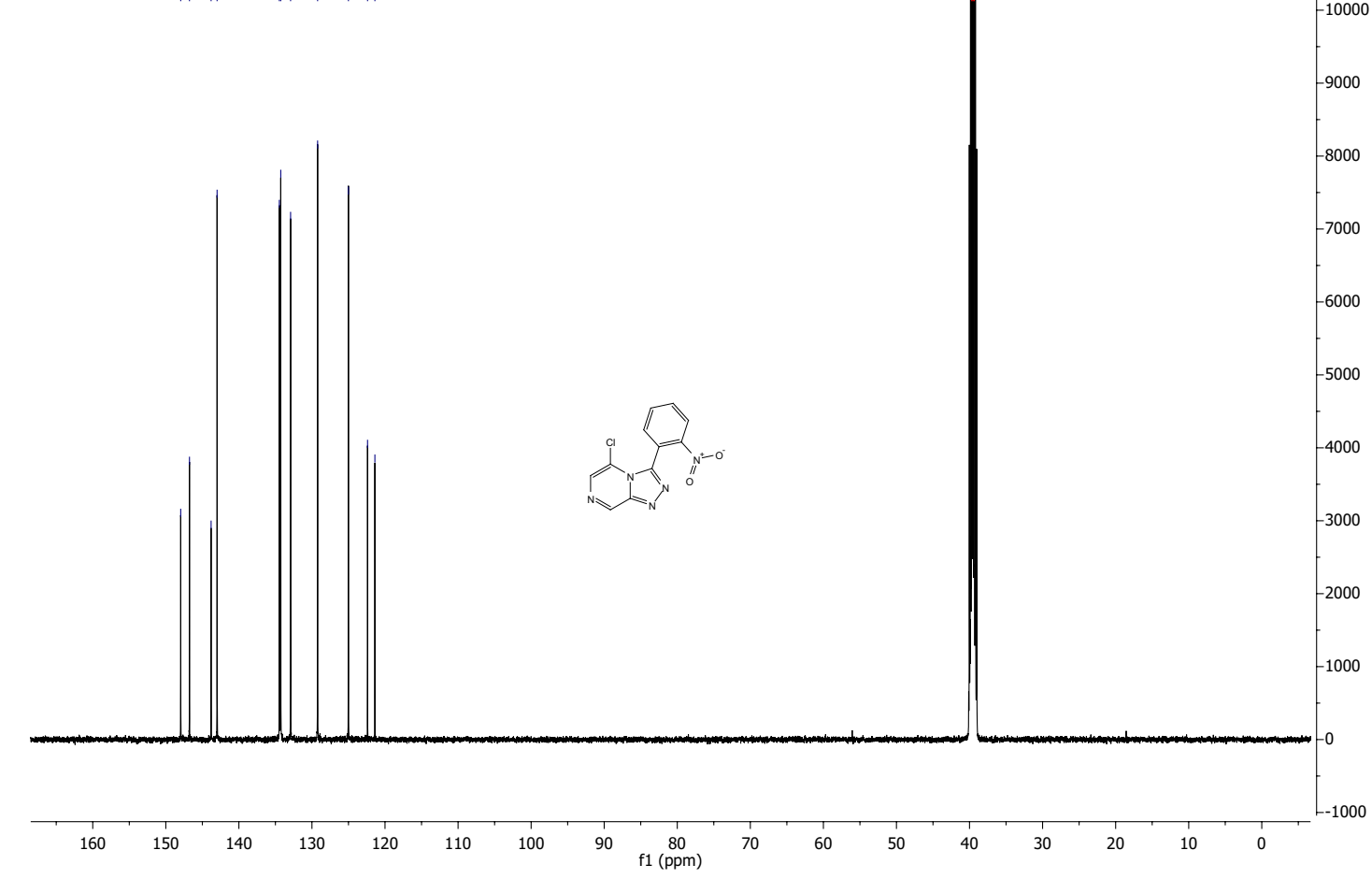

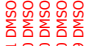


5-Chloro-3-(3,5-di-tert-butylphenyl)-[1,2,4]triazolo[4,3-a]pyrazine $45 f$
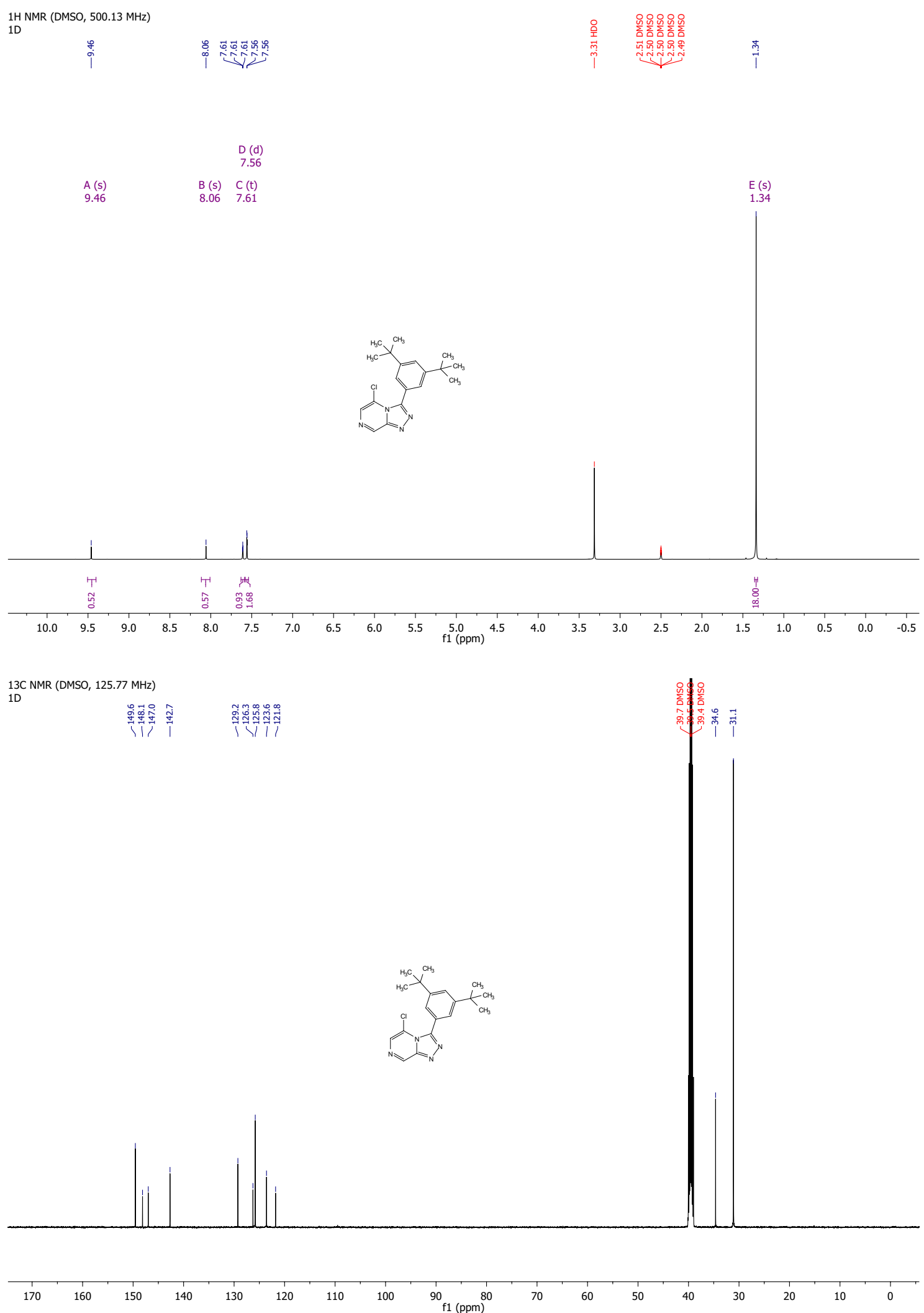
3-(Anthracen-9-yl)-5-chloro-[1,2,4] triazolo[4,3-a]pyrazine 45g

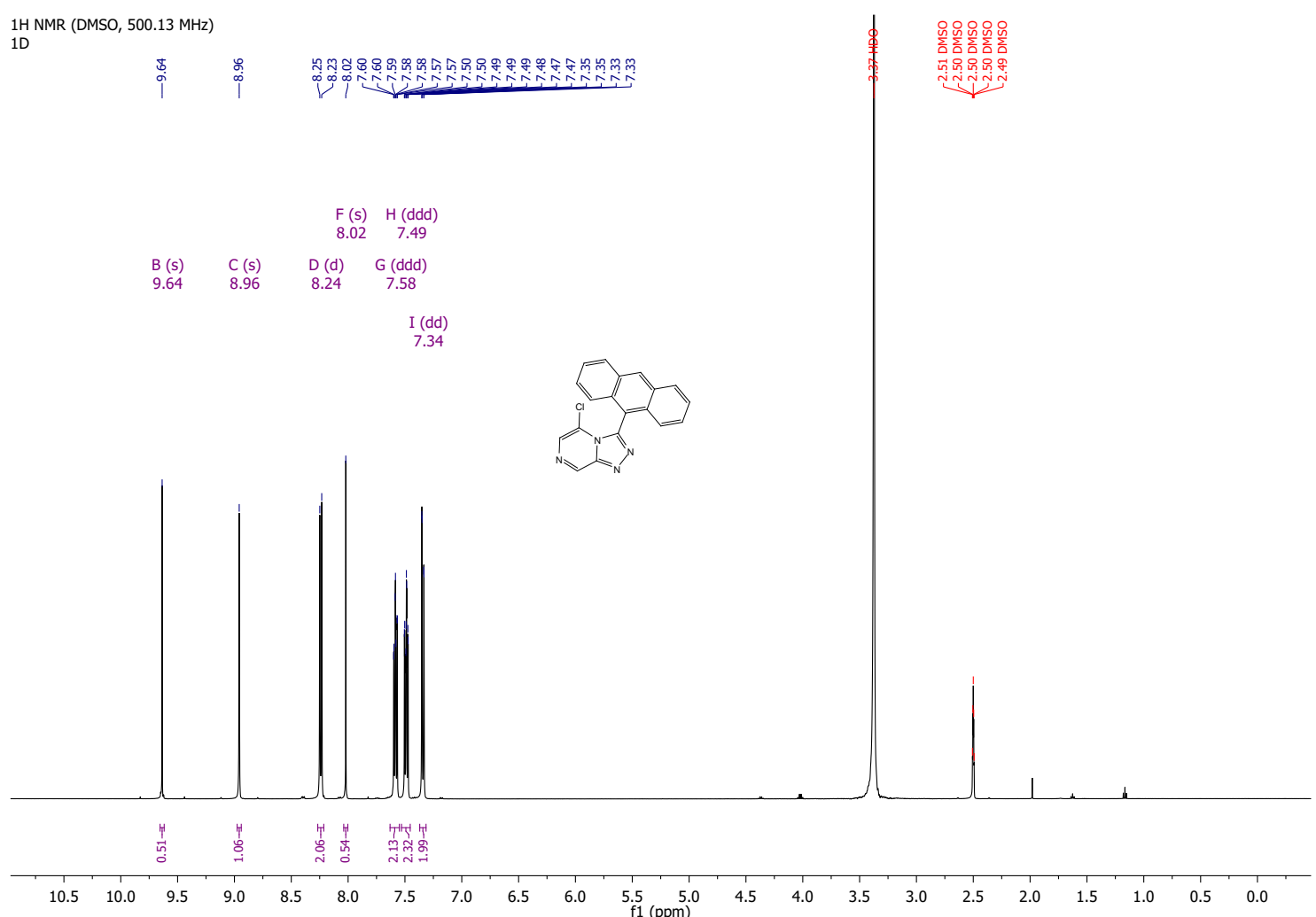

$\begin{array}{ll}\text { 13C NMR (DMSO, } 125.77 \mathrm{MHz}) & \\ 1 \mathrm{D} & \end{array}$

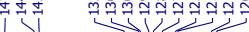

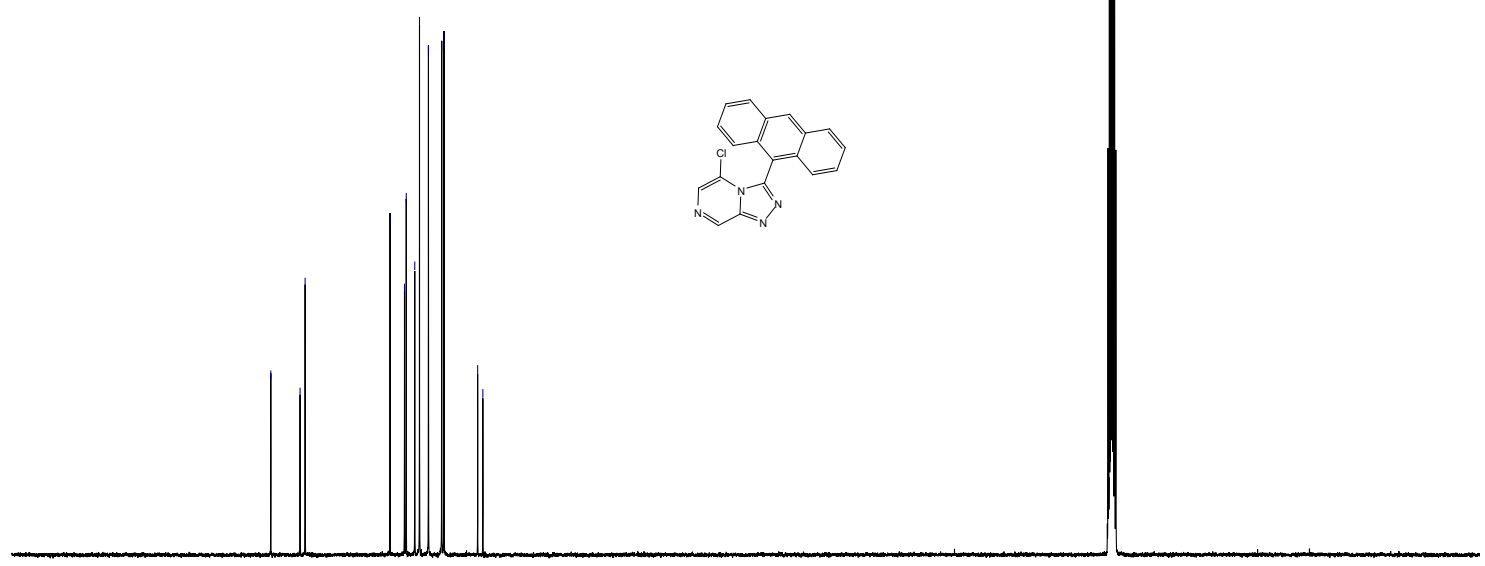

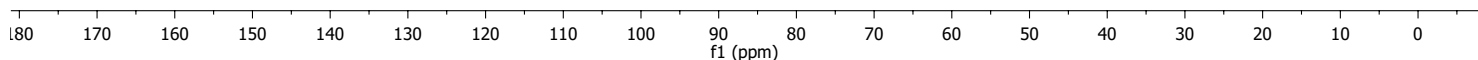


5-Bromo-[1,2,4]triazolo[4,3-a]pyrazine $45 \mathrm{~h}$

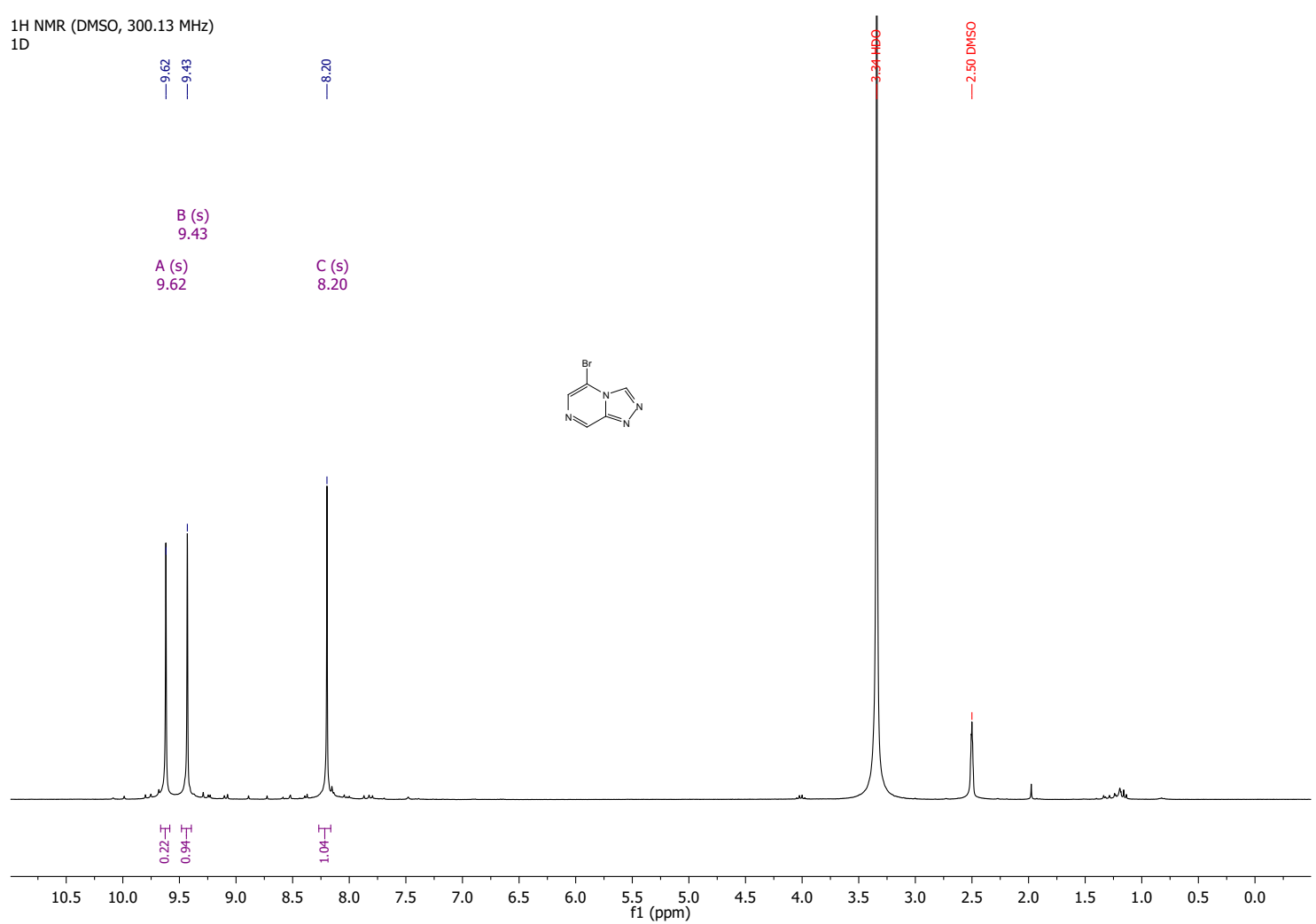

13C NMR (DMSO, $75.48 \mathrm{MHz}$ )
1D

।

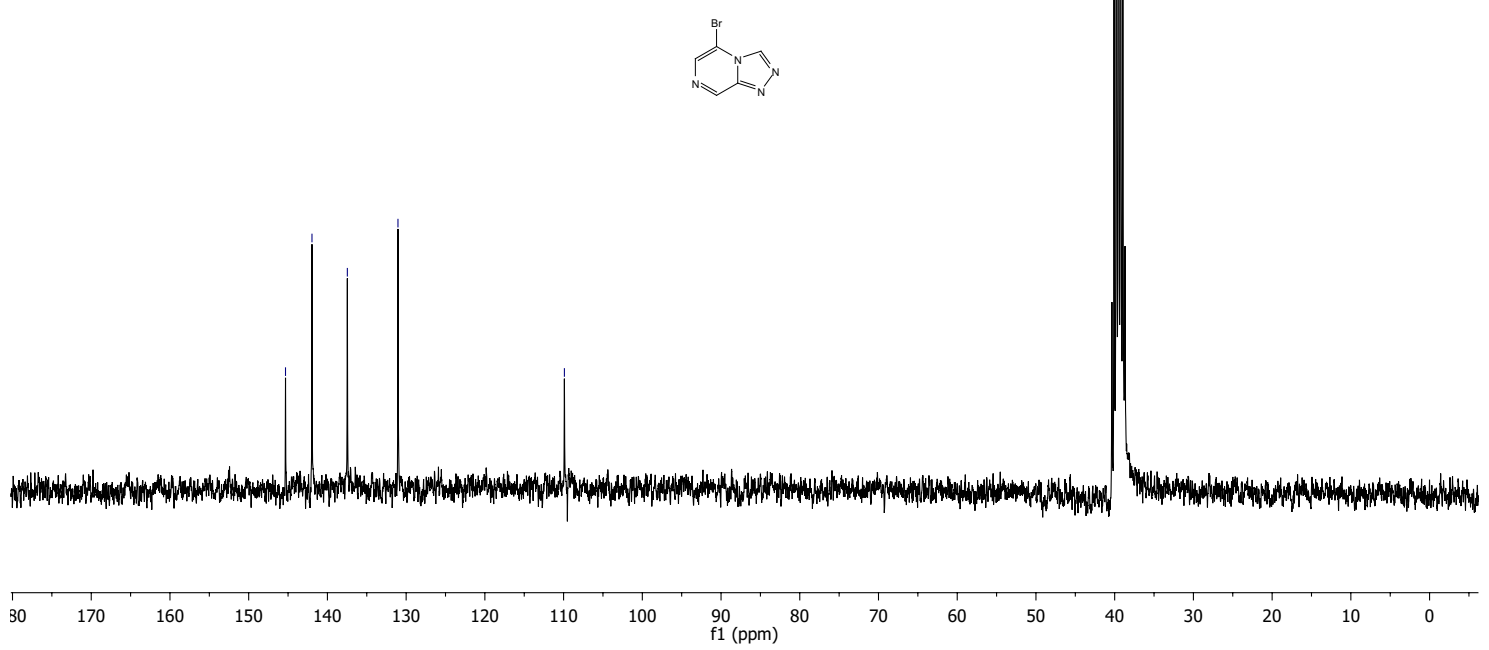


5-Bromo-3-(4-methoxyphenyl)-[1,2,4]triazolo[4,3-a]pyrazine $45 \mathrm{i}$

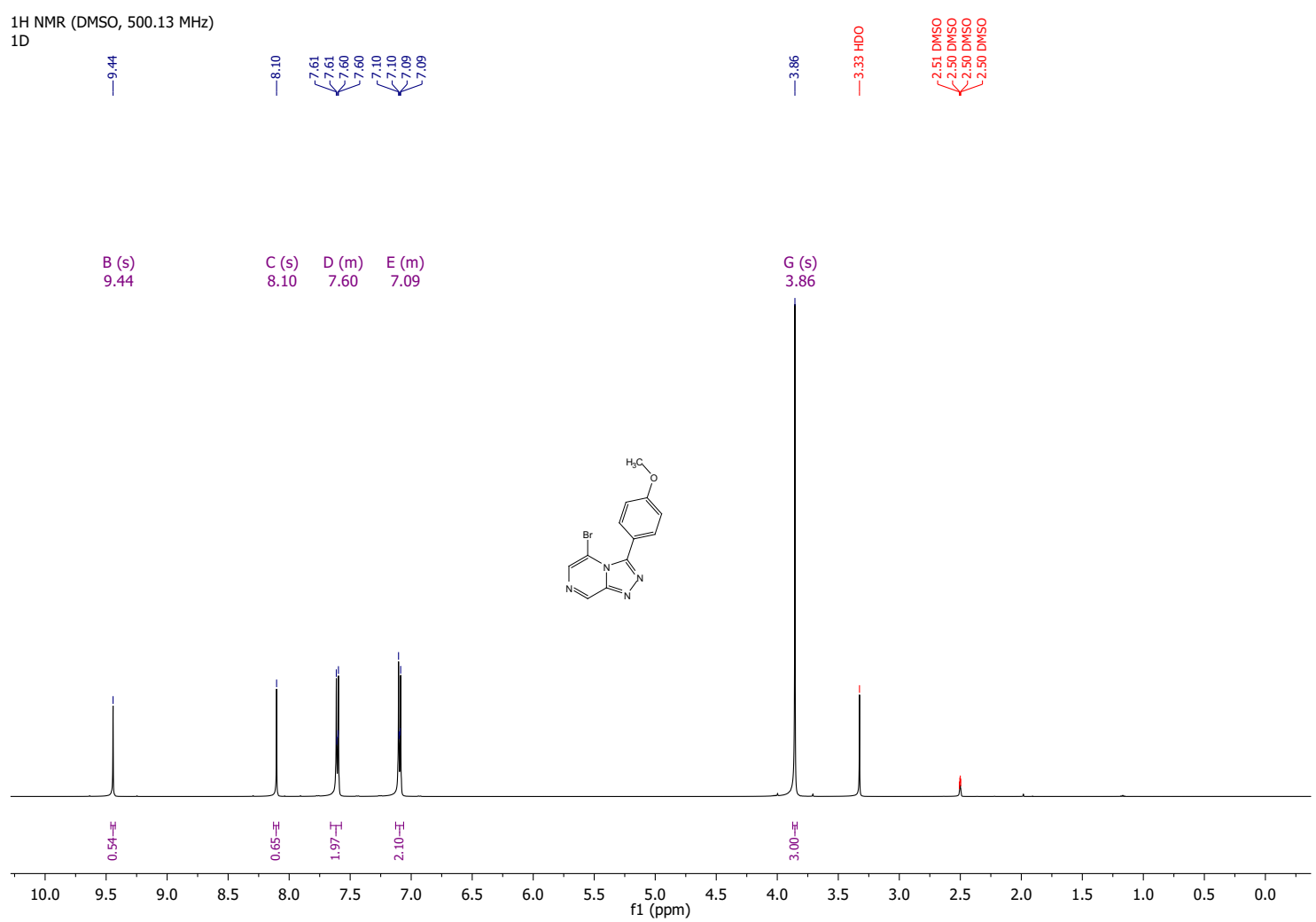

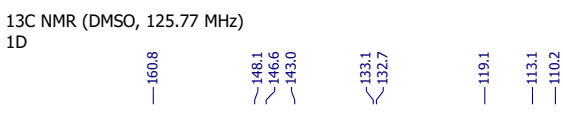

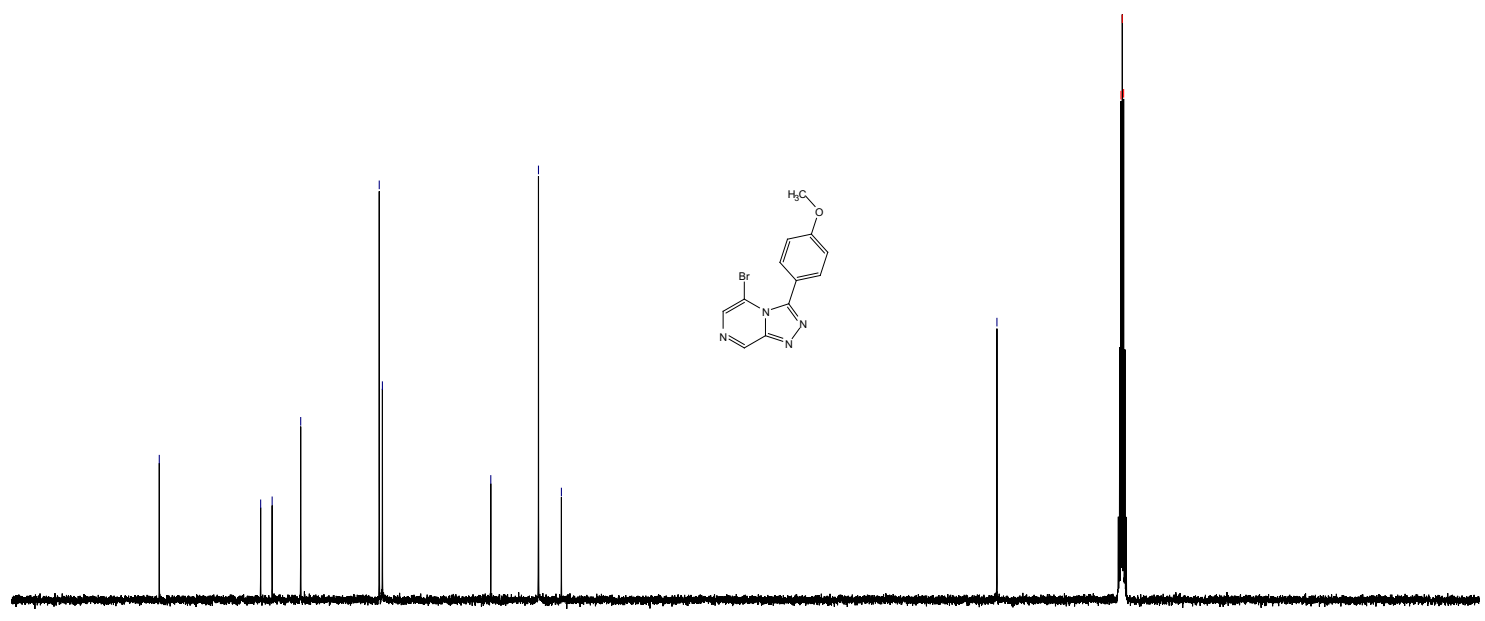


5-Bromo-3-(4-nitrophenyl)-[1,2,4]triazolo[4,3-a]pyrazine 45j

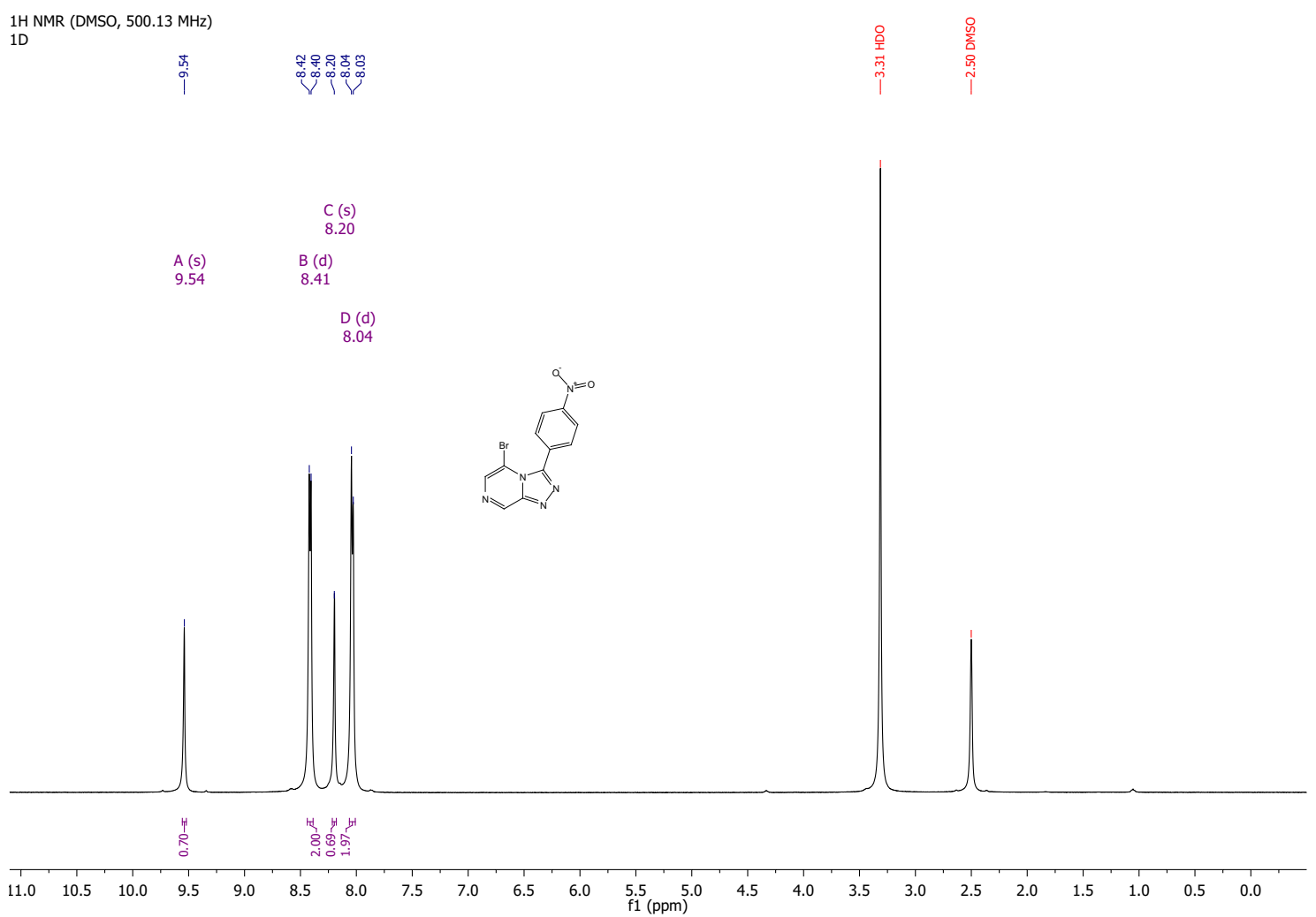

13C NMR (DMSO, $125.77 \mathrm{MHz}$ )
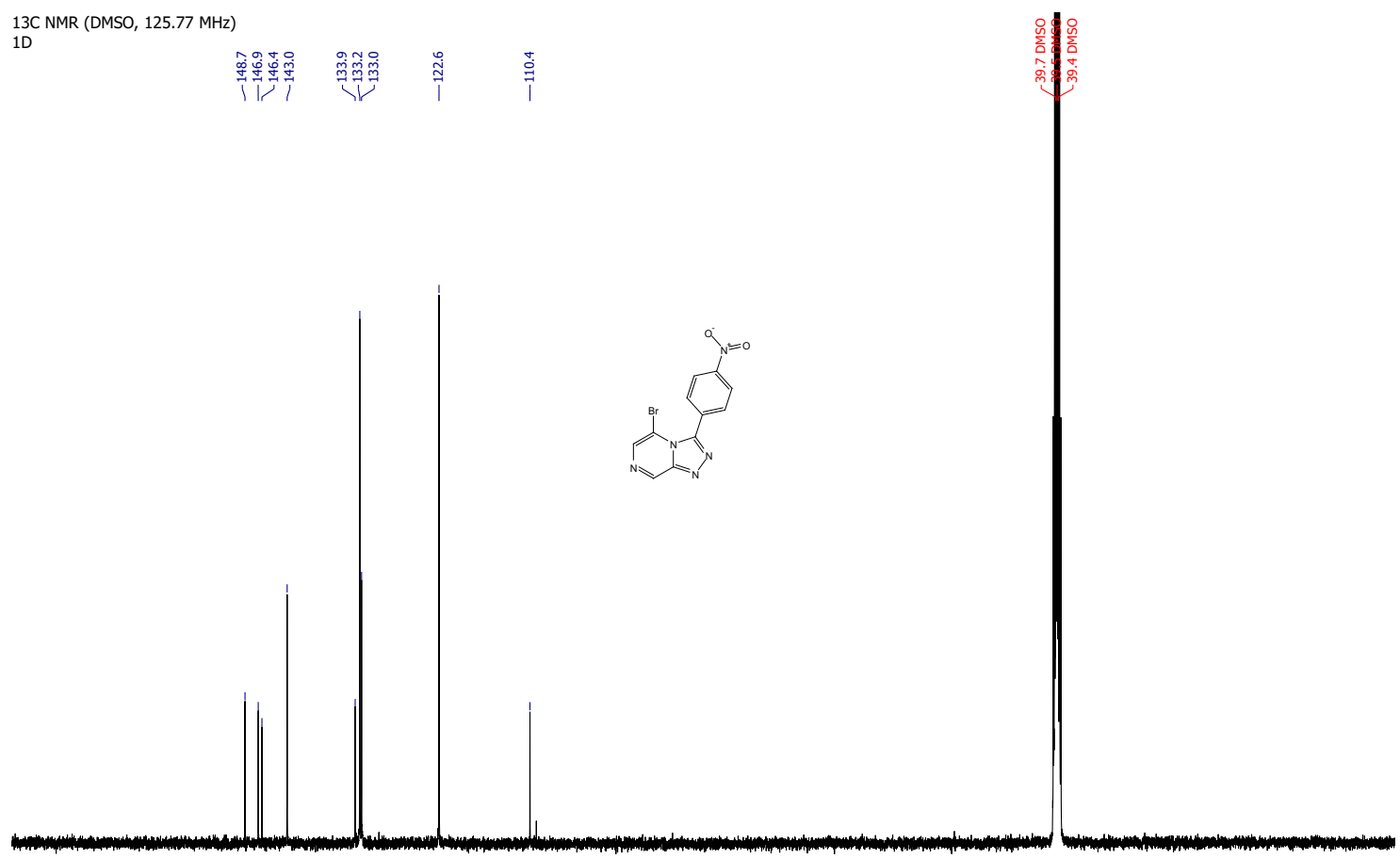
5-Bromo-3-(2-methoxyphenyl)-[1,2,4] triazolo[4,3-a]pyrazine $45 \mathrm{k}$

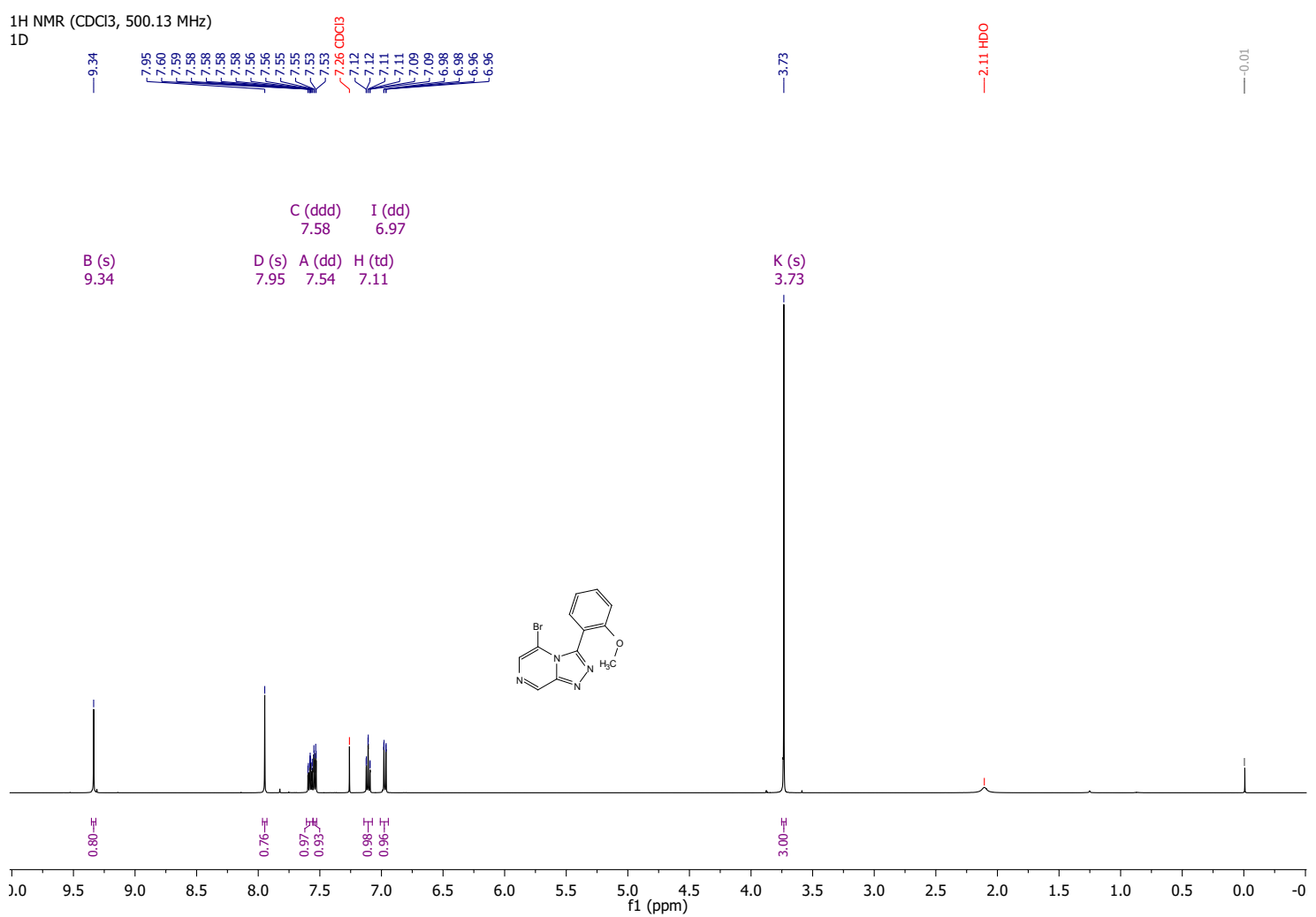

${ }_{10}^{13 \mathrm{C} N M R}(\mathrm{CDCl} 3,125.77 \mathrm{MHz})$
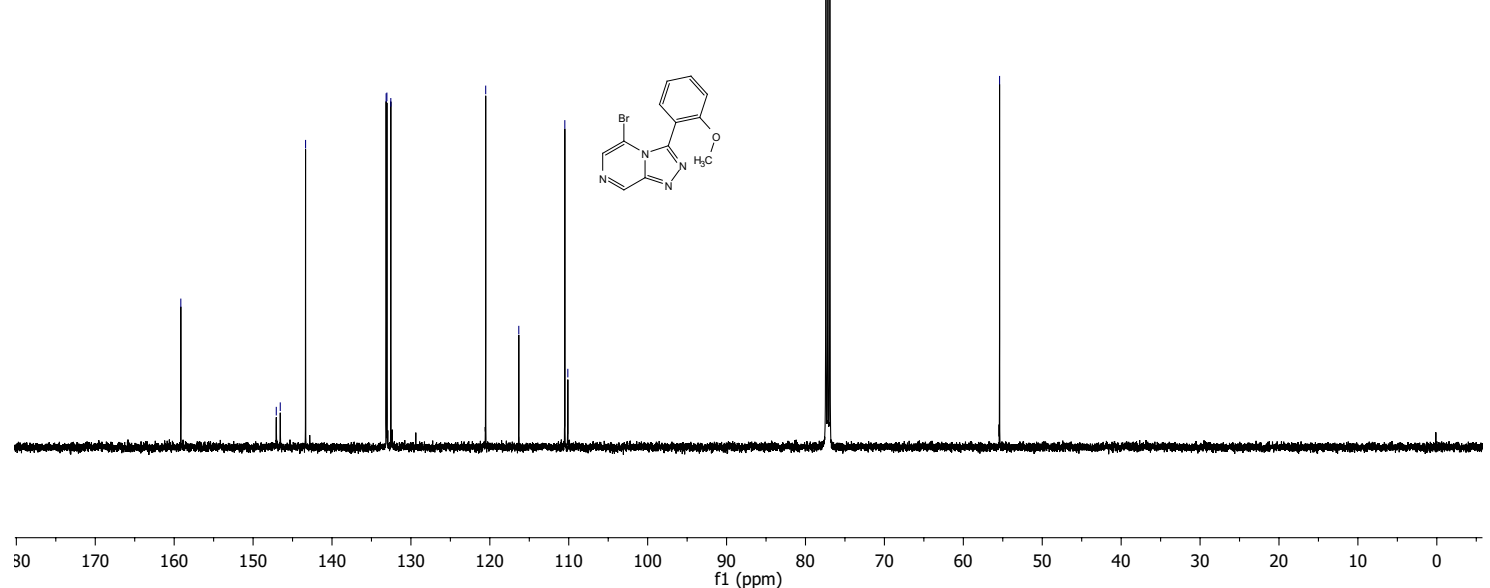
5-Bromo-3-(2-nitrophenyl)-[1,2,4] triazolo[4,3-a]pyrazine 451

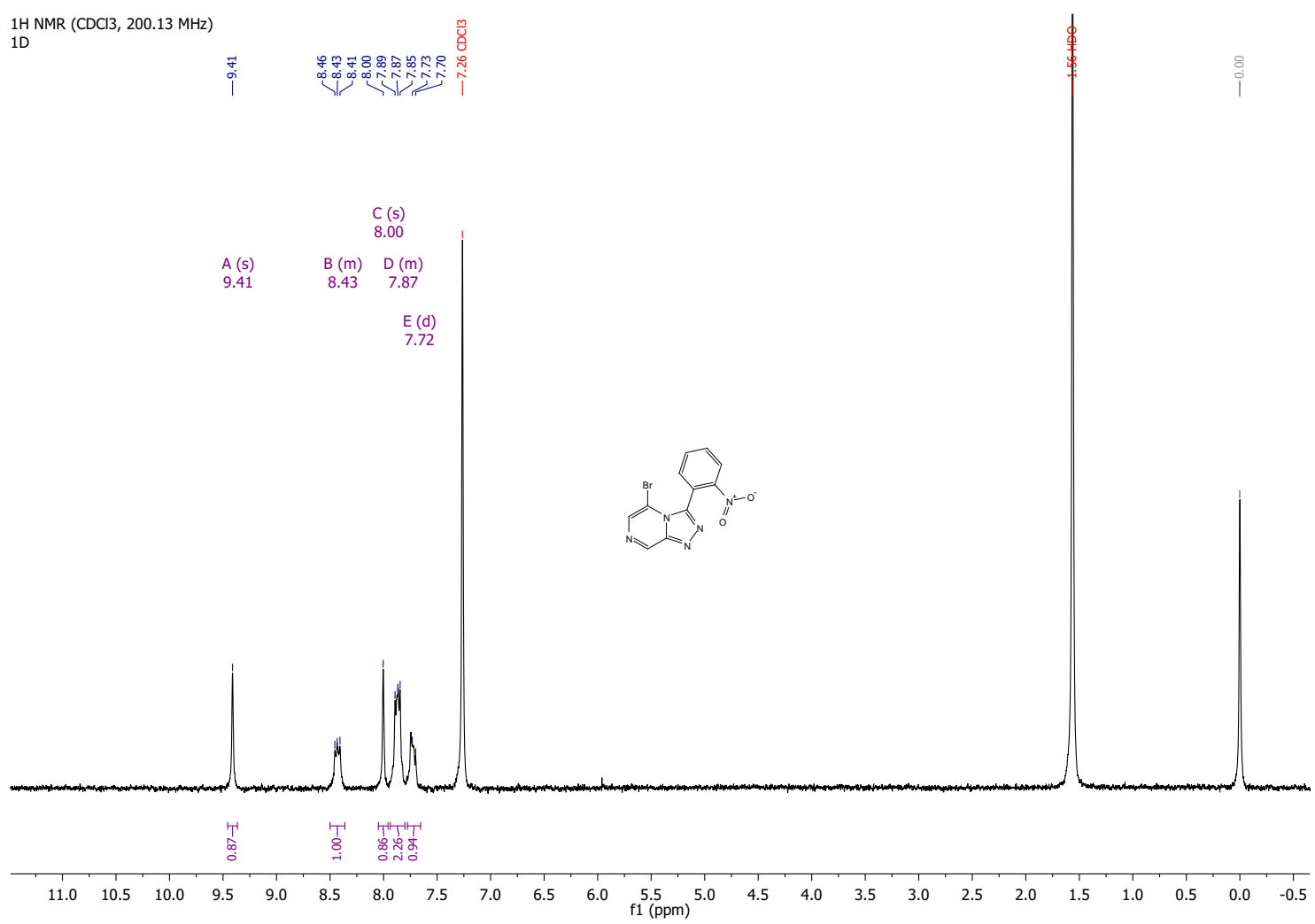

13C NMR (DMSO, $125.77 \mathrm{MHz}$ )

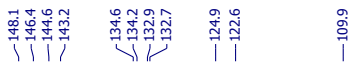
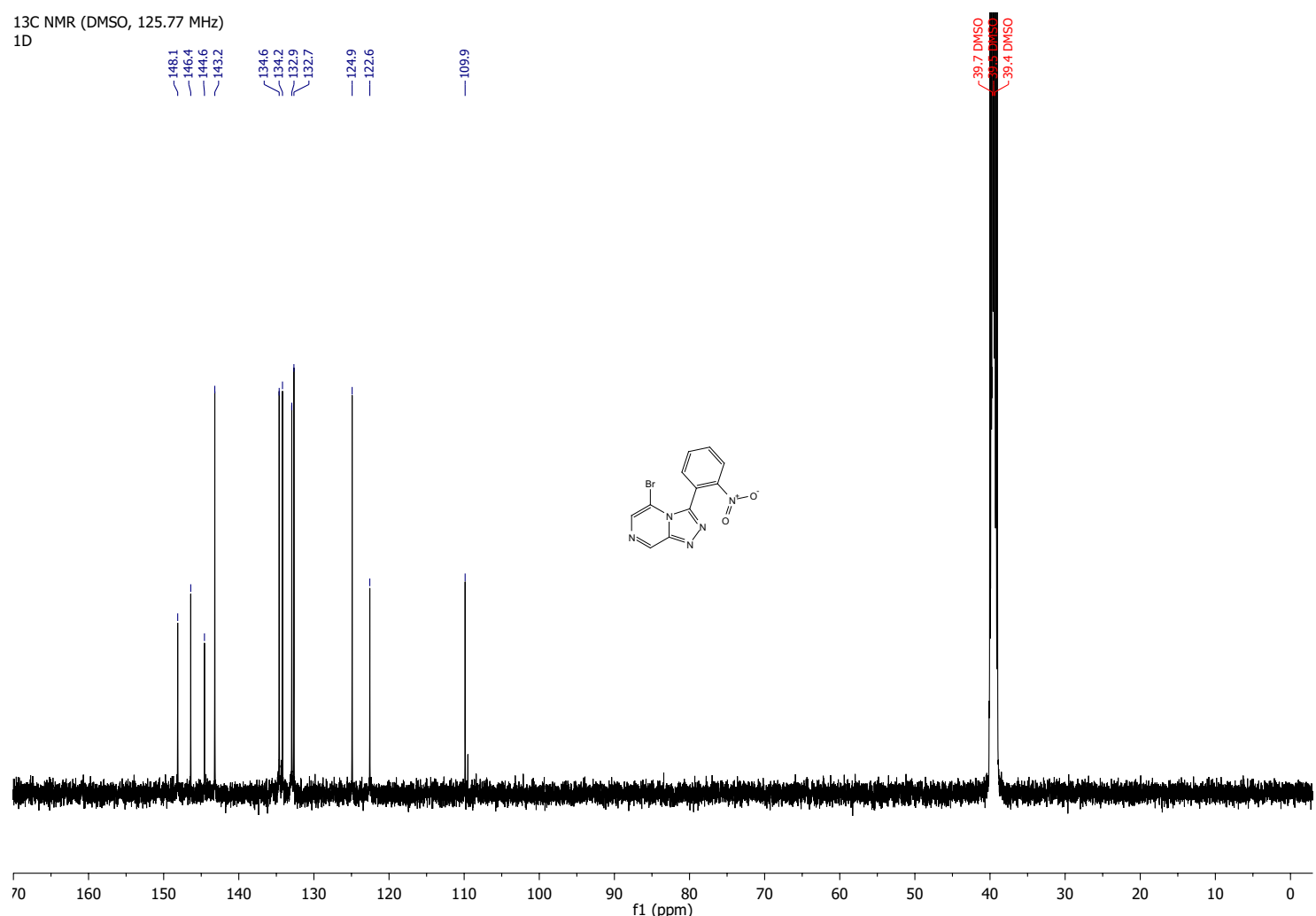


\section{5-Iodo-[1,2,4] triazolo[4,3-a]pyrazine $45 \mathrm{~m}$}
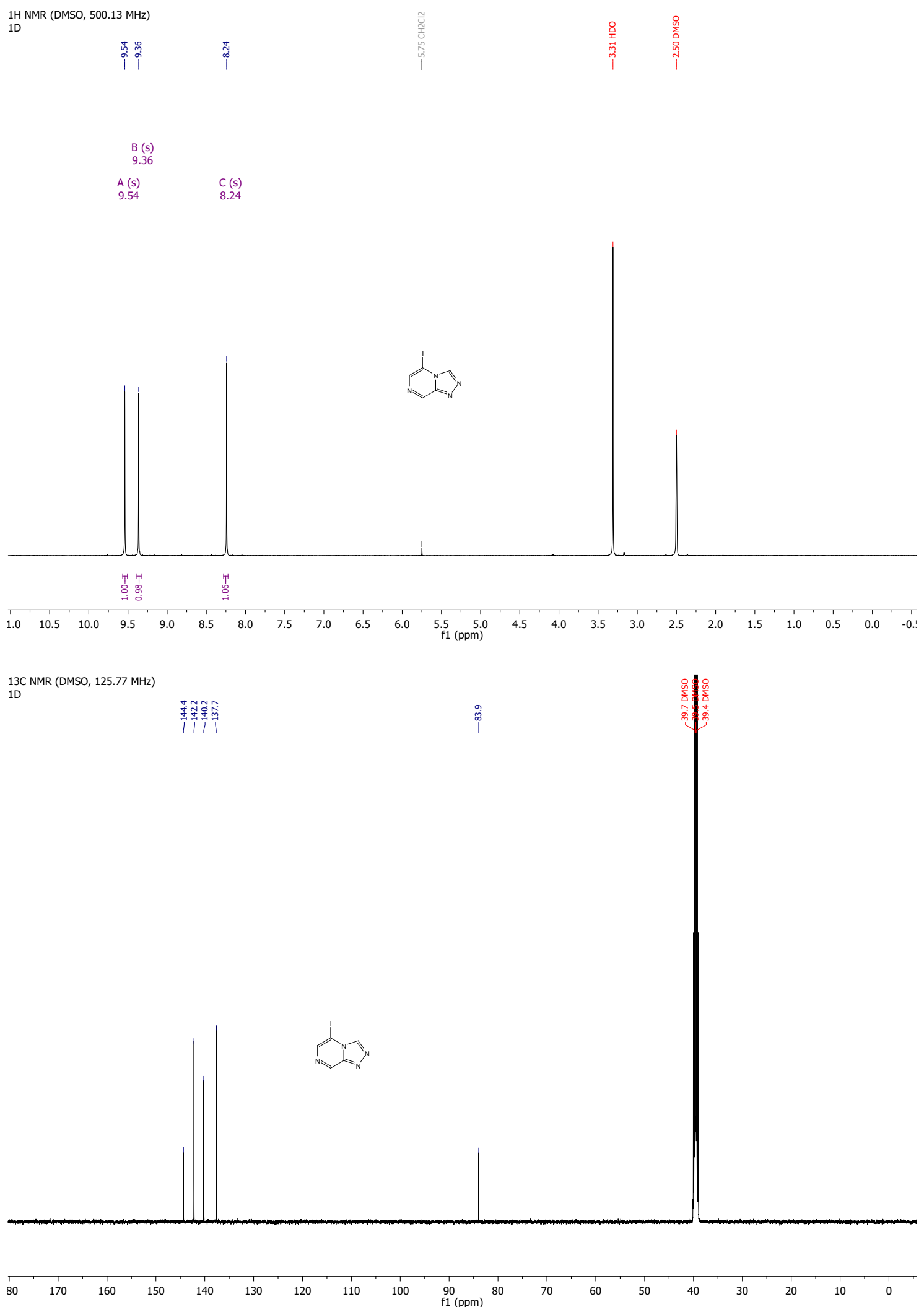
5-Iodo-3-(4-methoxyphenyl)-[1,2,4]triazolo[4,3-a]pyrazine 45n

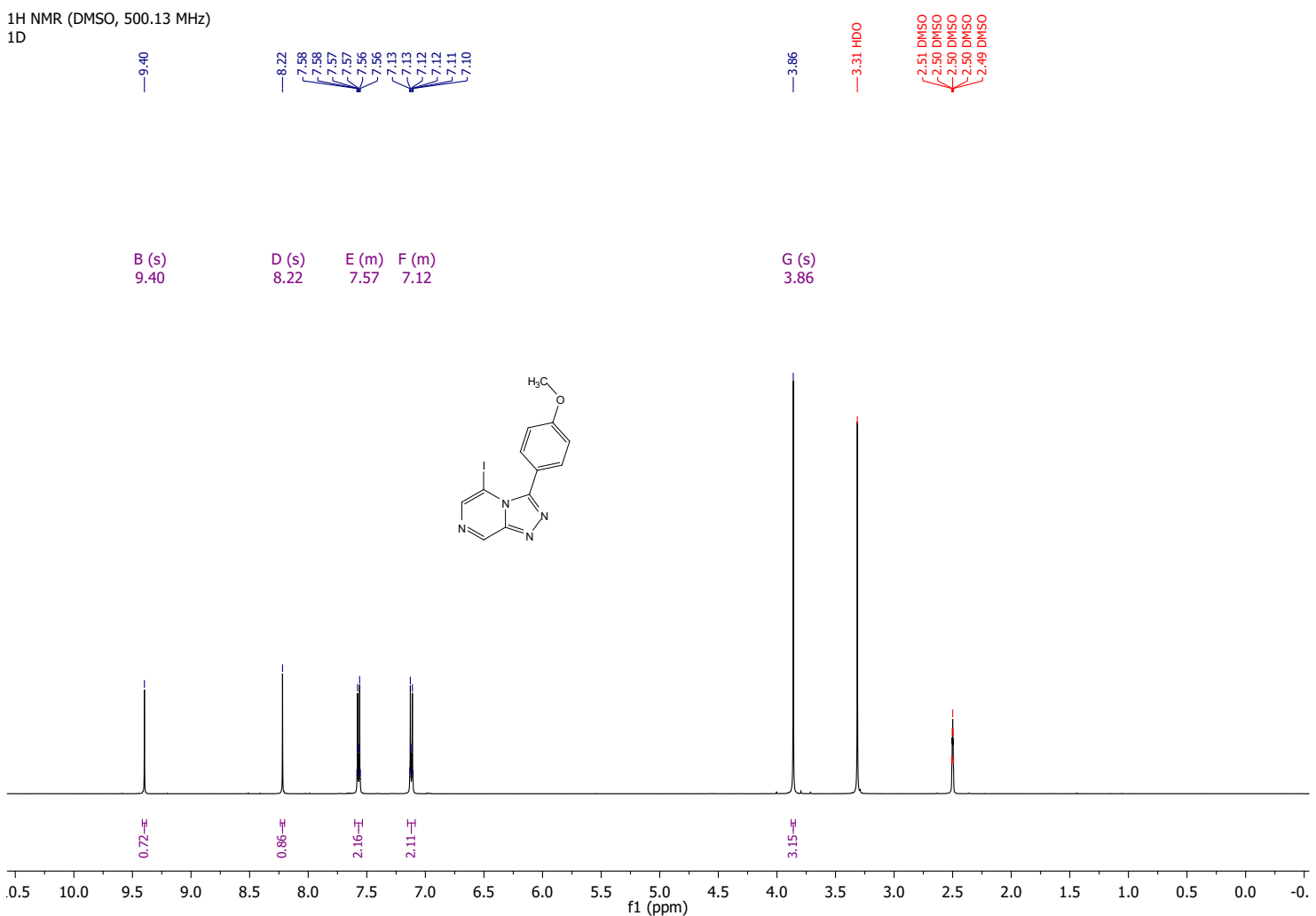

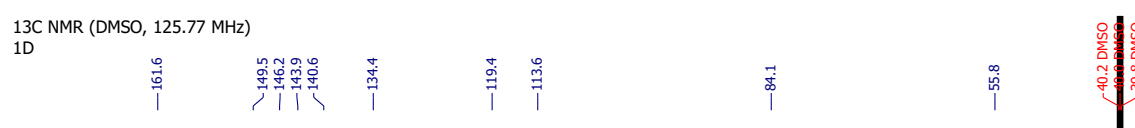

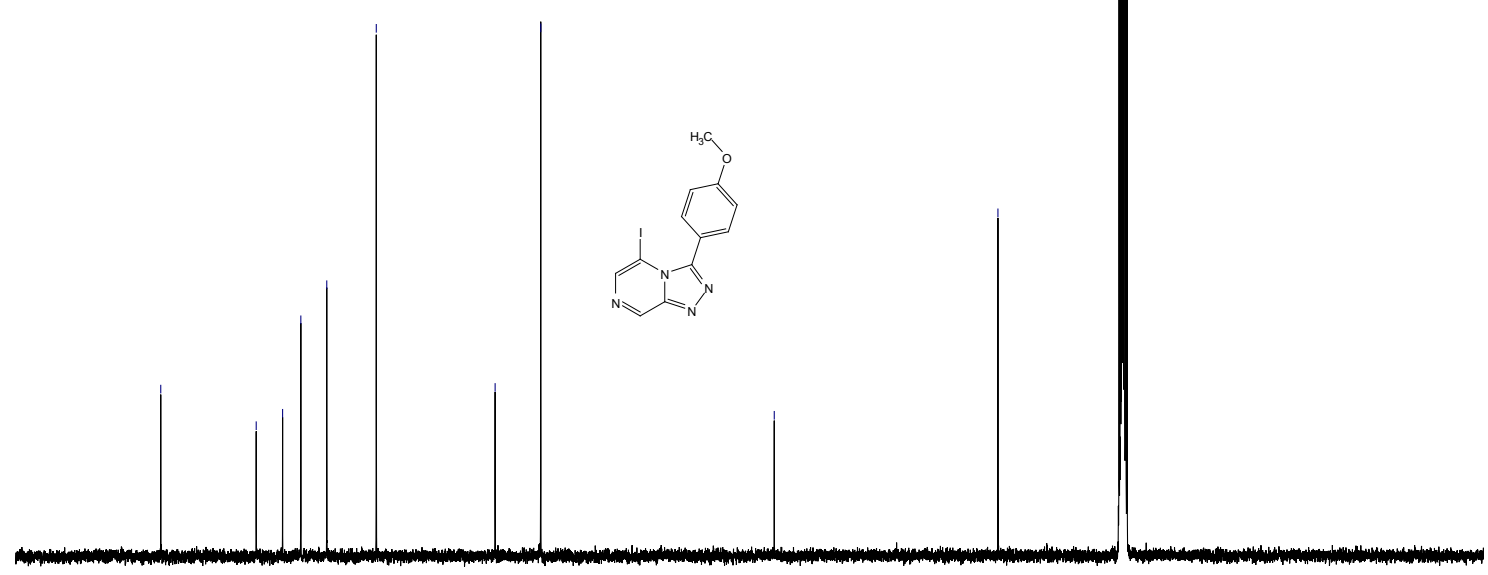




\section{5-Phenethoxy-[1,2,4] triazolo[4,3-a]pyrazine 46a}
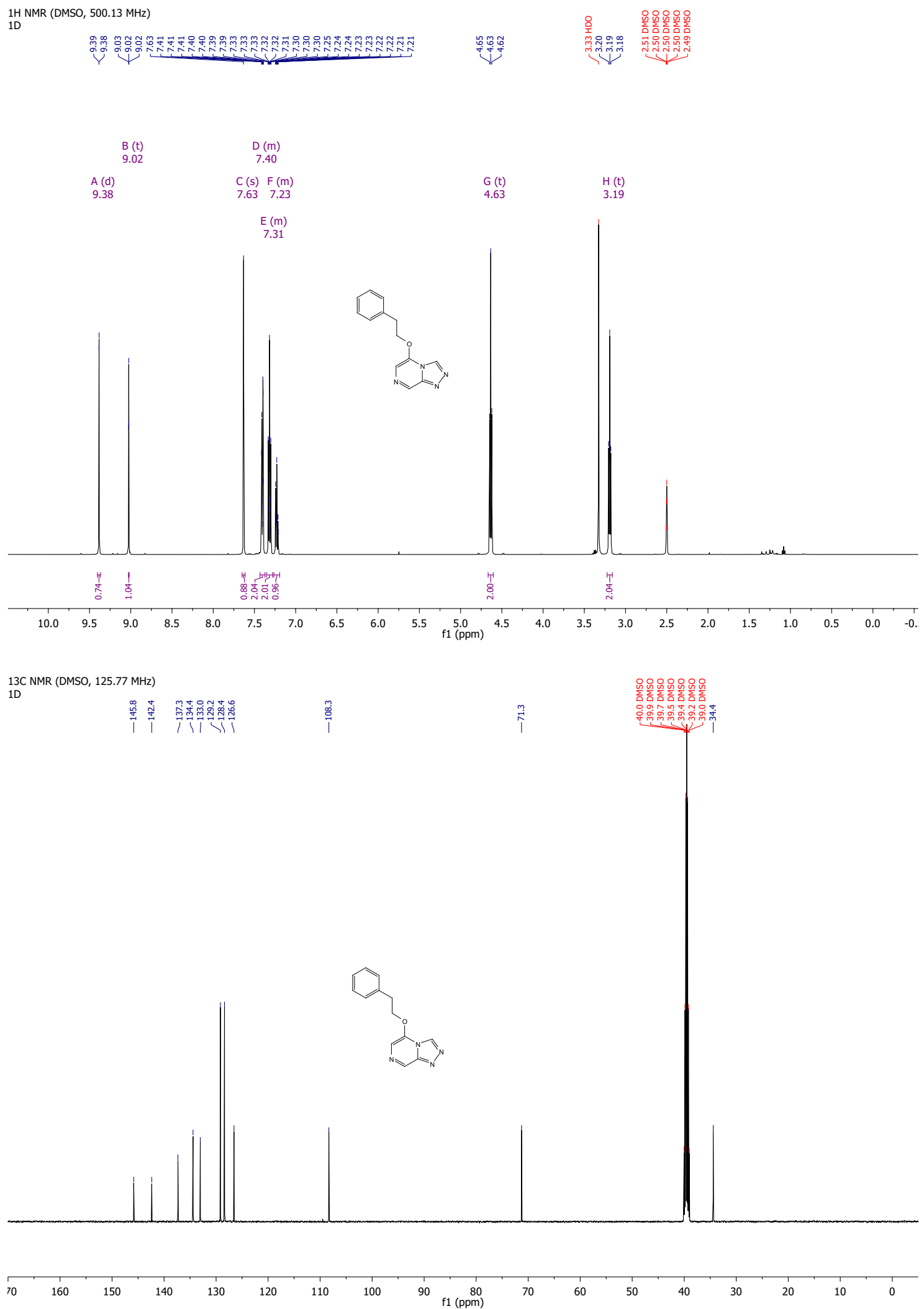
5-(Phenethylthio)-[1,2,4] triazolo[4,3-a]pyrazine 46b

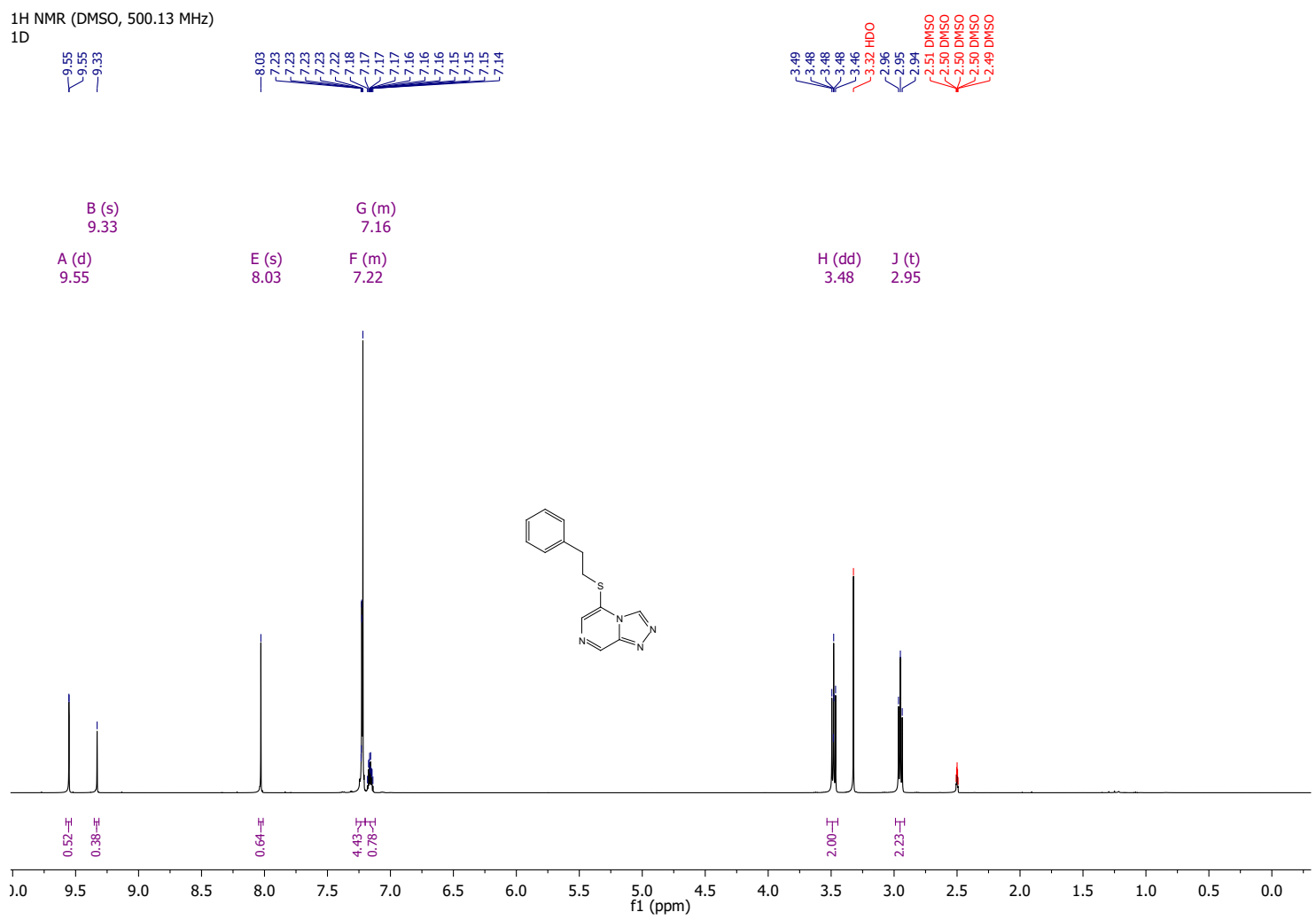

13C NMR (DMSO, $125.77 \mathrm{MHz}$ )
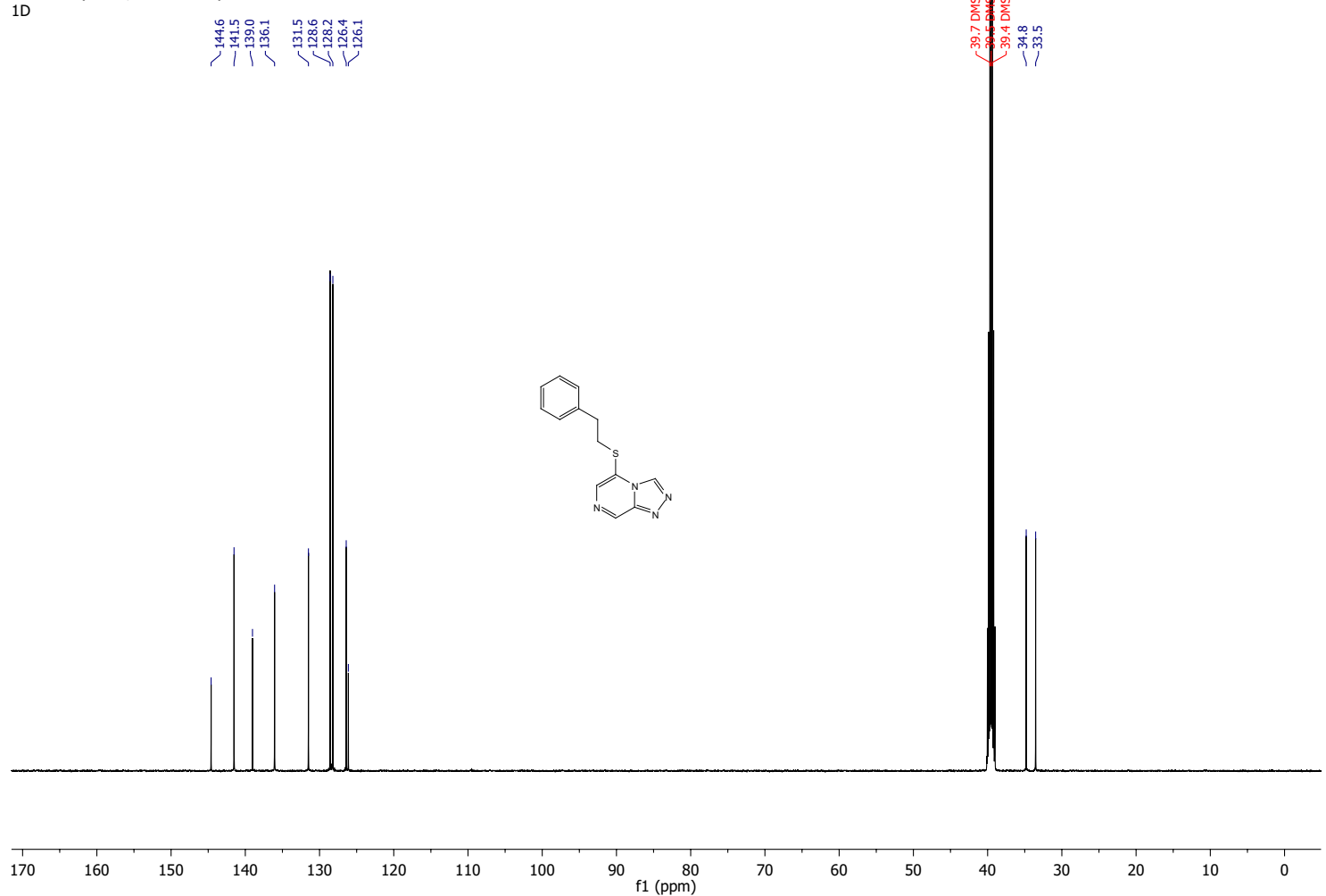
3-(4-Methoxyphenyl)-5-phenethoxy-[1,2,4]triazolo[4,3-a]pyrazine 46c

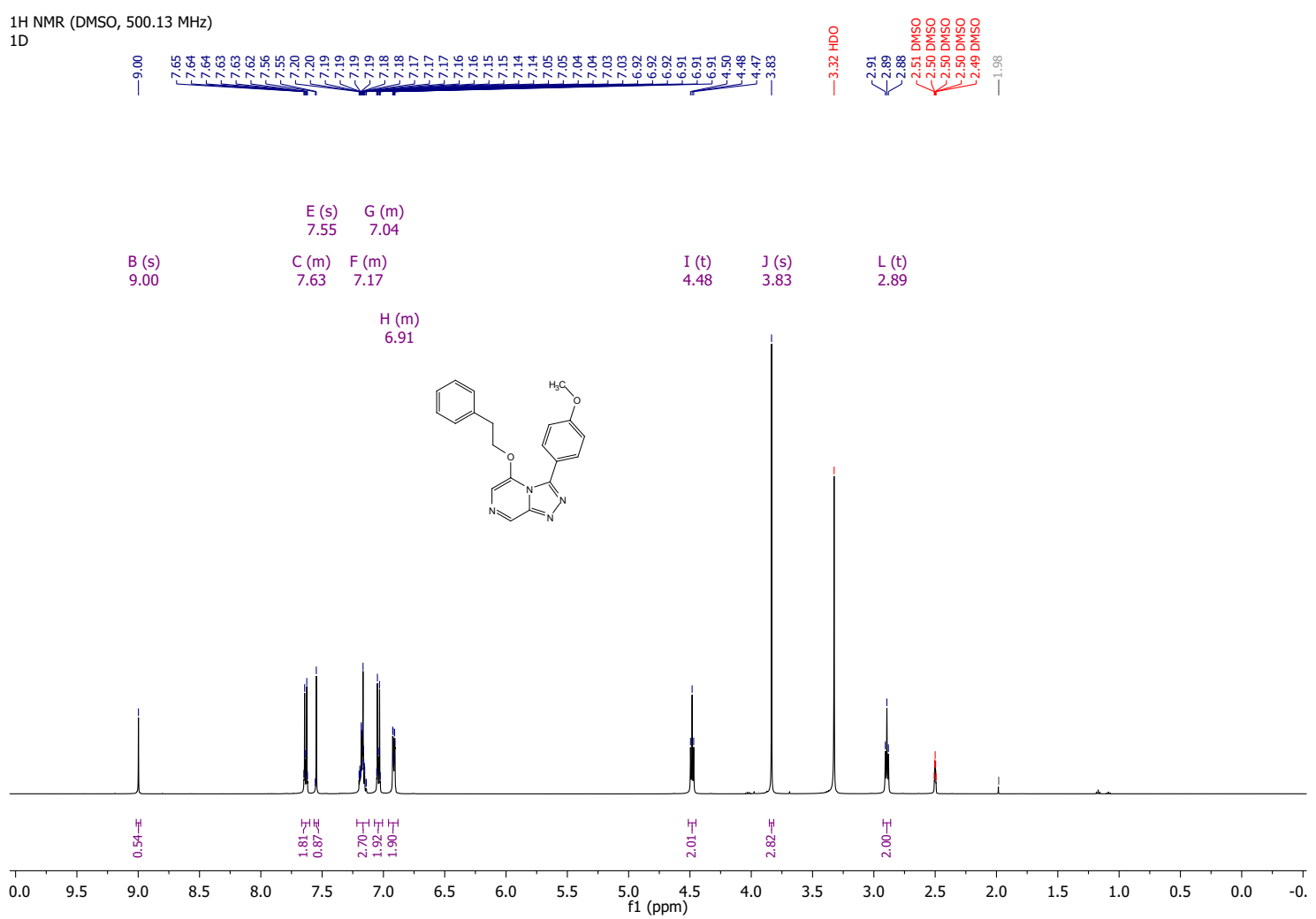

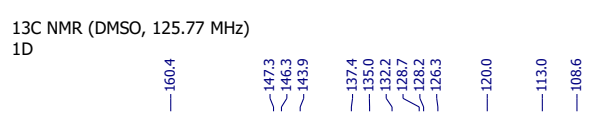

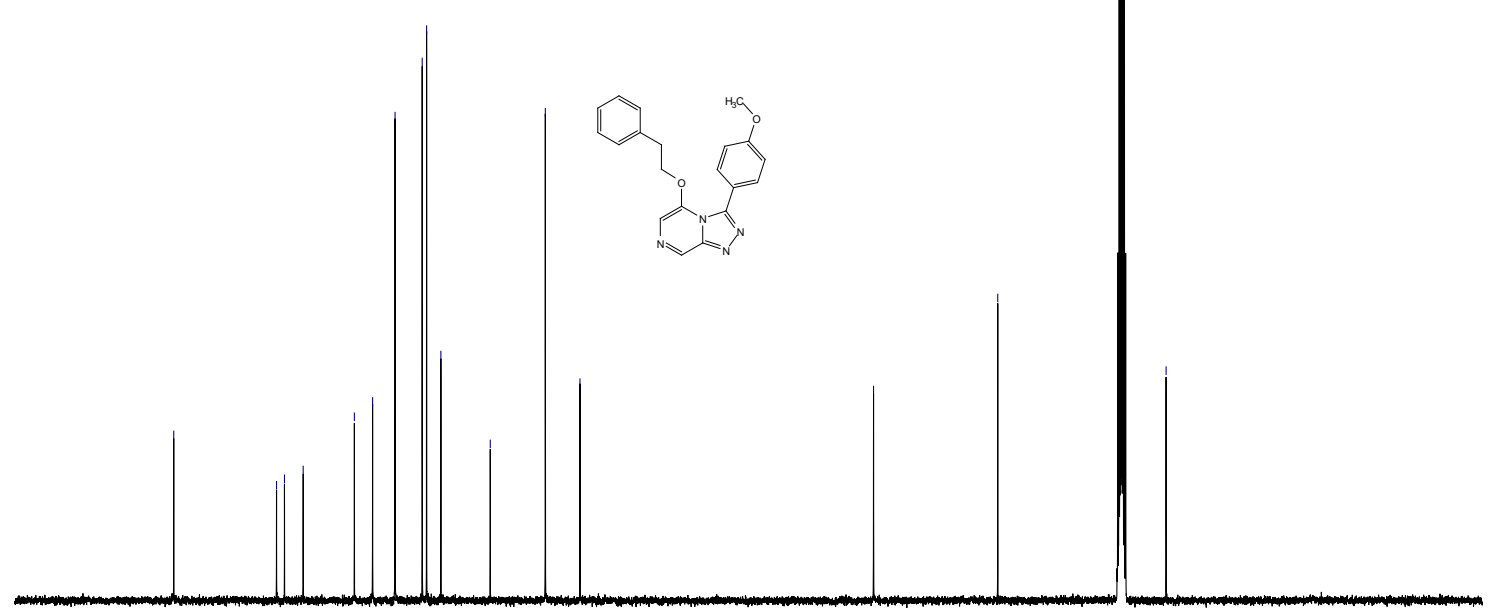

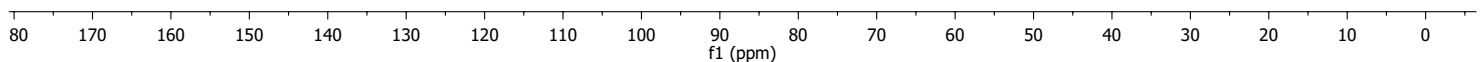


3-(4-Methoxyphenyl)-5-(phenethylthio)-[1,2,4]triazolo[4,3-a]pyrazine 46d
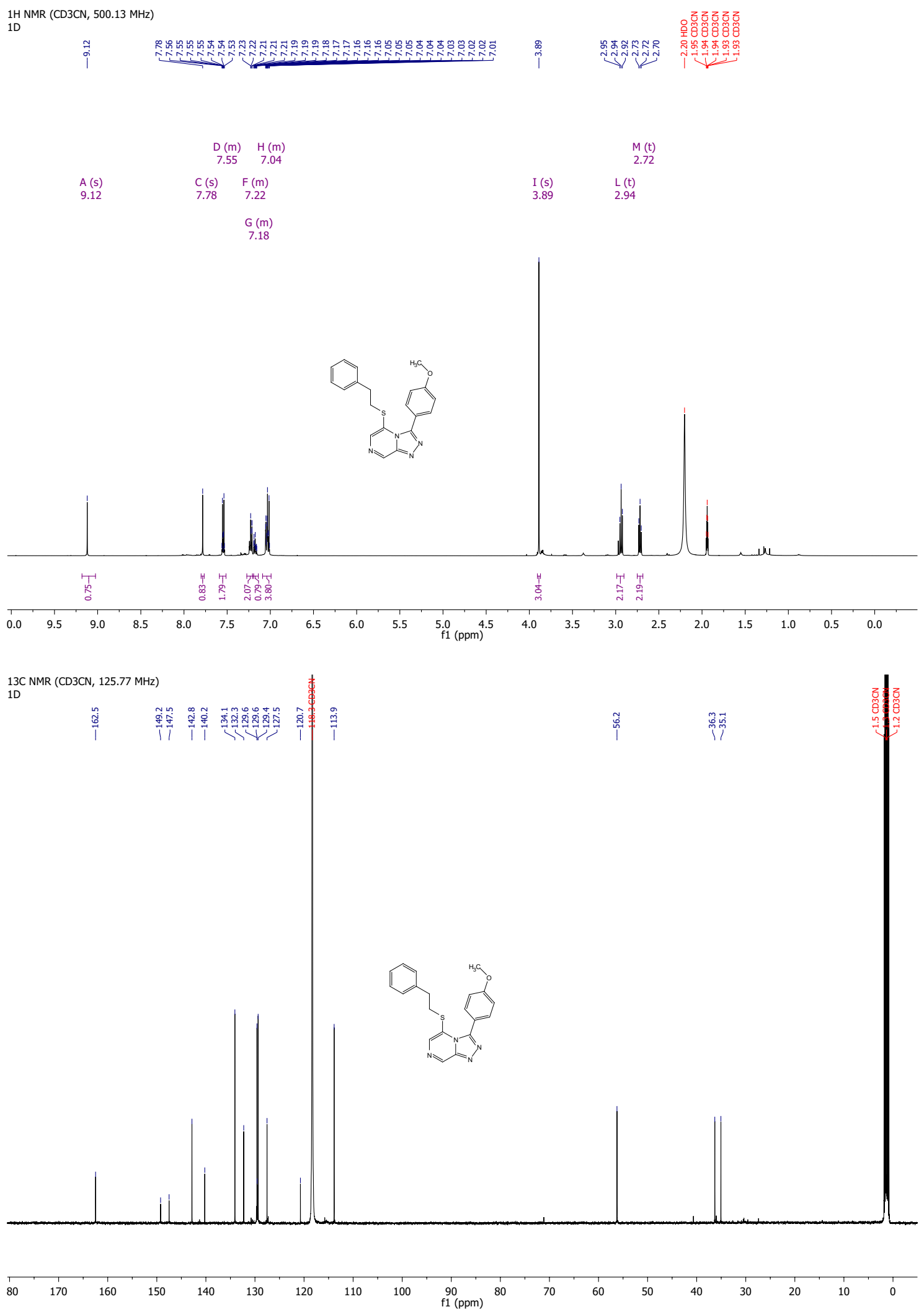
3-(4-Nitrophenyl)-5-phenethoxy-[1,2,4]triazolo[4,3-a]pyrazine 46e
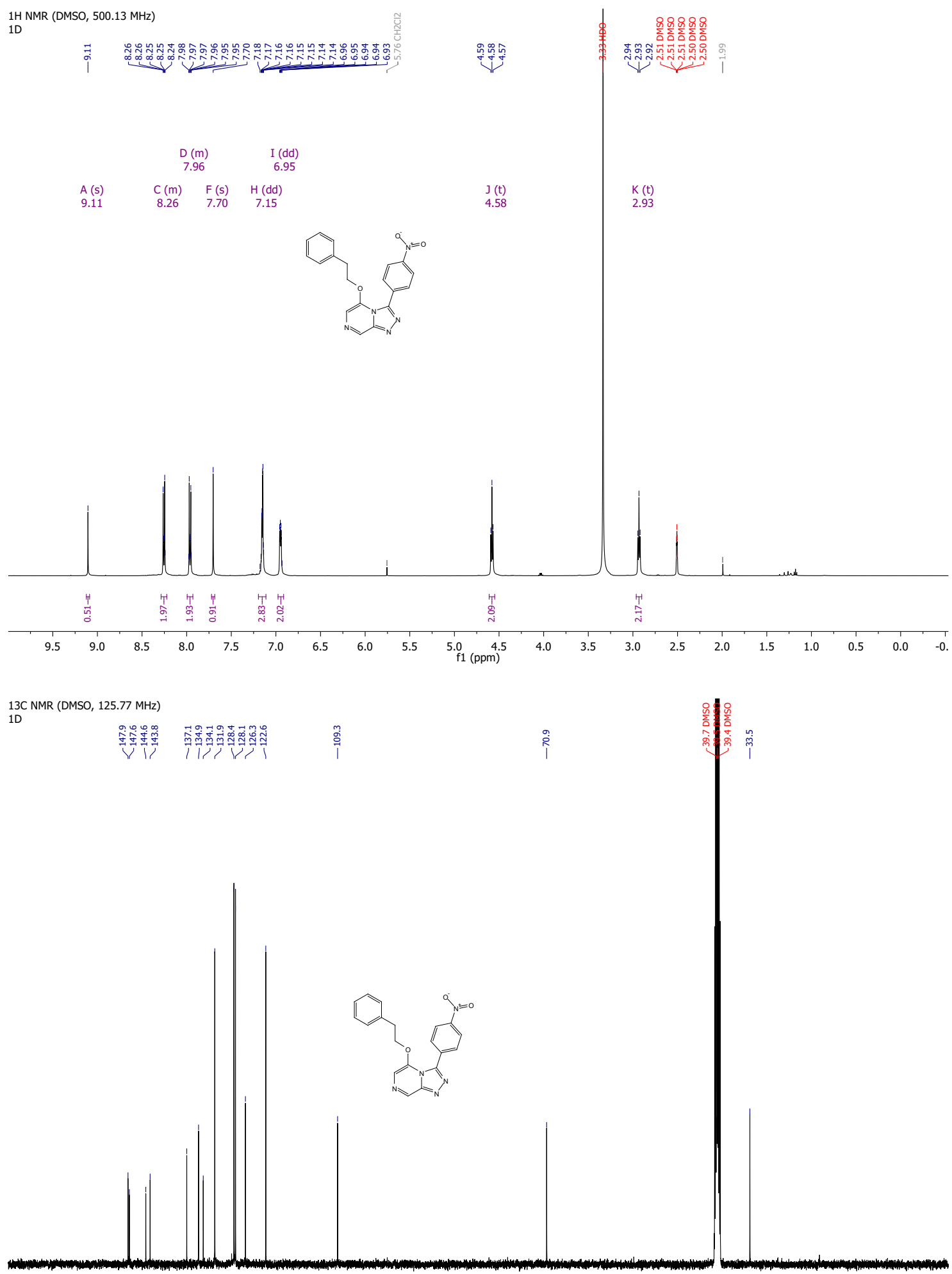
3-(2-Methoxyphenyl)-5-phenethoxy-[1,2,4]triazolo[4,3-a]pyrazine $46 f$
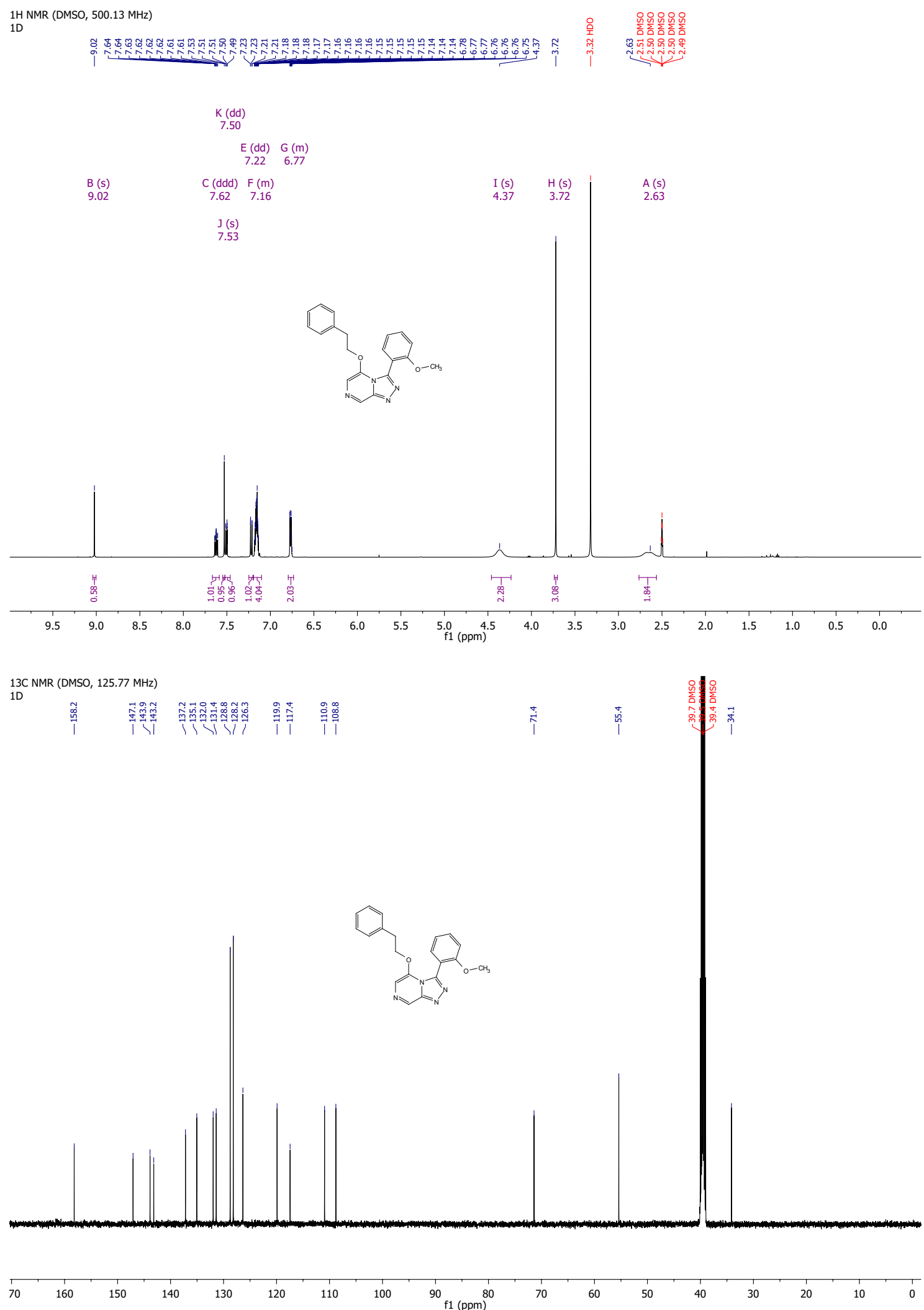
3-(2-Nitrophenyl)-5-phenethoxy-[1,2,4]triazolo[4,3-a]pyrazine $46 \mathrm{~g}$

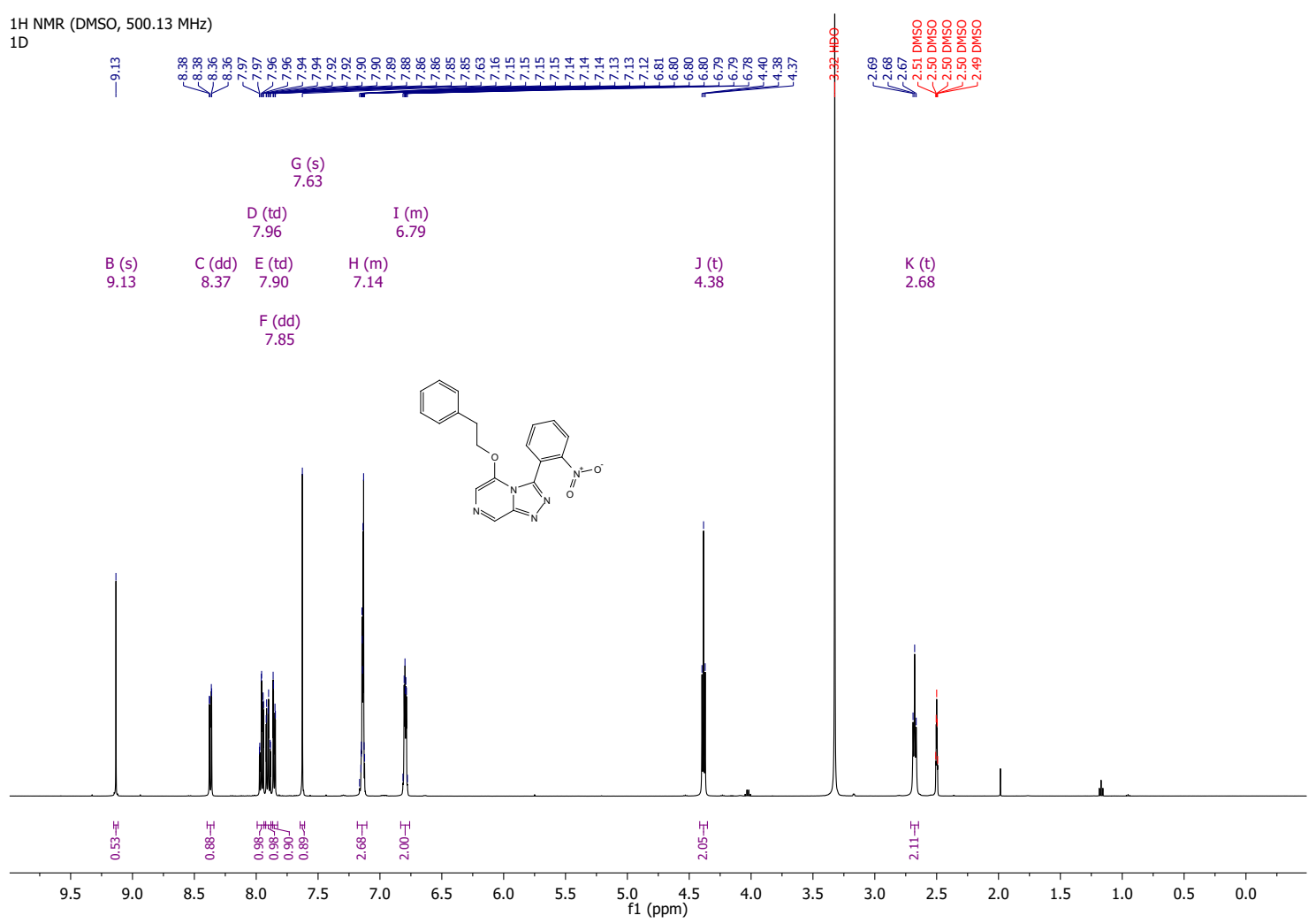

$13 \mathrm{C}$ NMR (DMSO, $125.77 \mathrm{MHz})$
$1 \mathrm{D}$
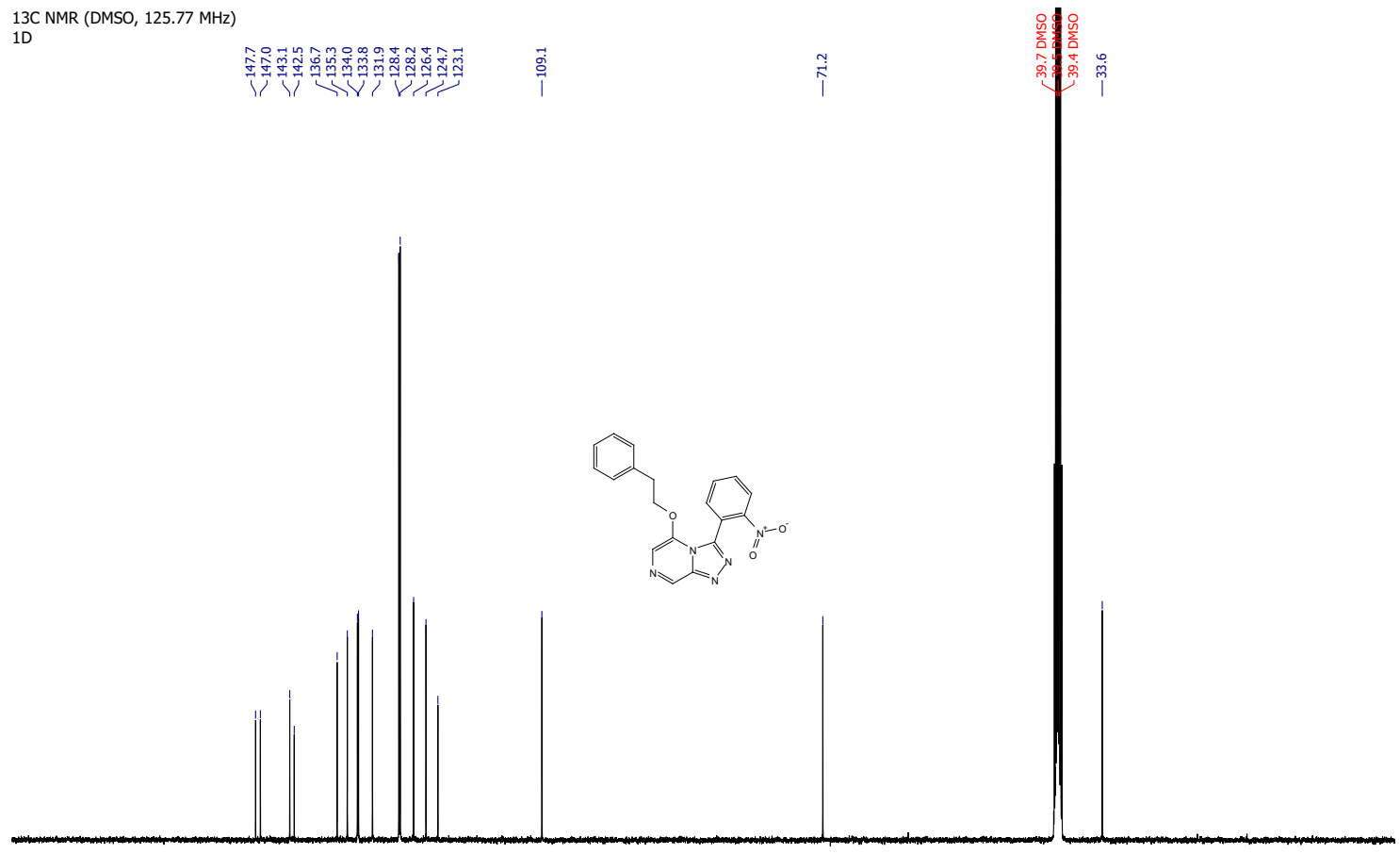

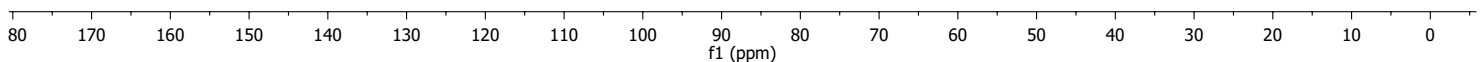


3-(3,5-Di-tert-butylphenyl)-5-phenethoxy-[1,2,4]triazolo[4,3-a]pyrazine $46 \mathrm{~h}$
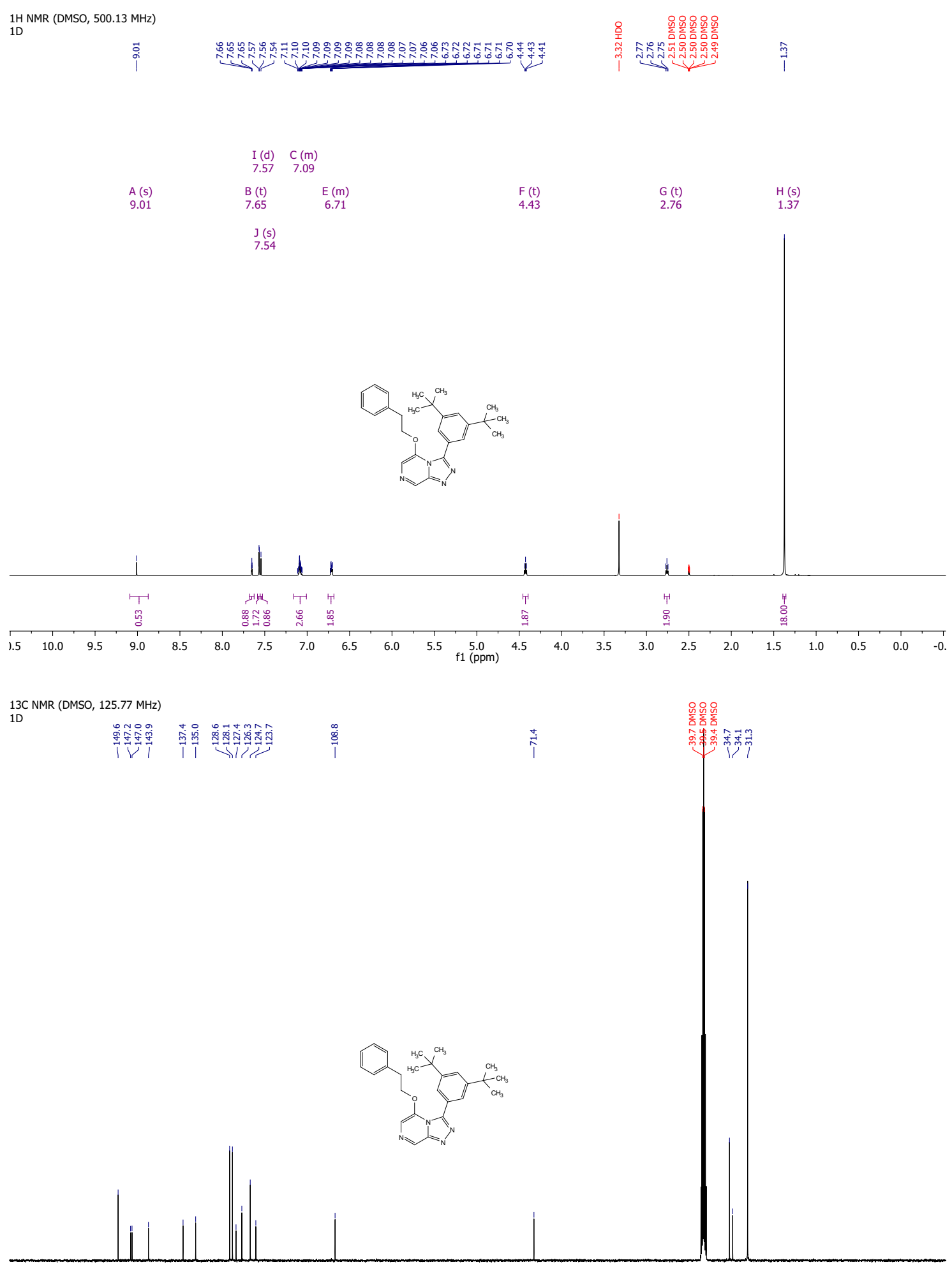

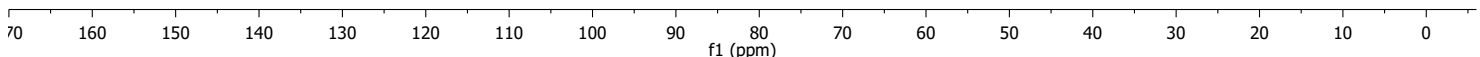


3-(Anthracen-9-yl)-5-phenethoxy-[1,2,4]triazolo[4,3-a]pyrazine 46i

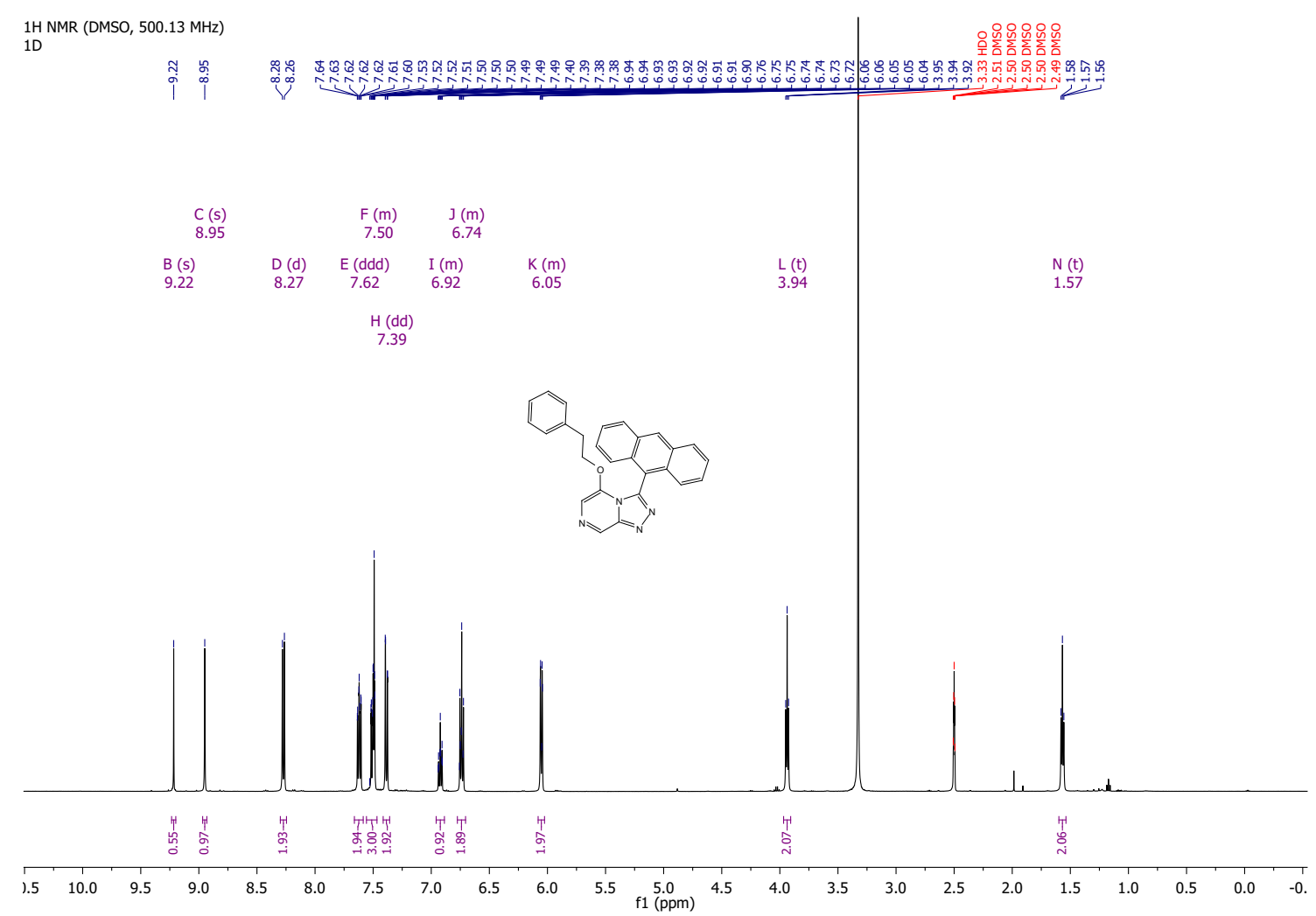

13C NMR (DMSO, 125.77 MHz)
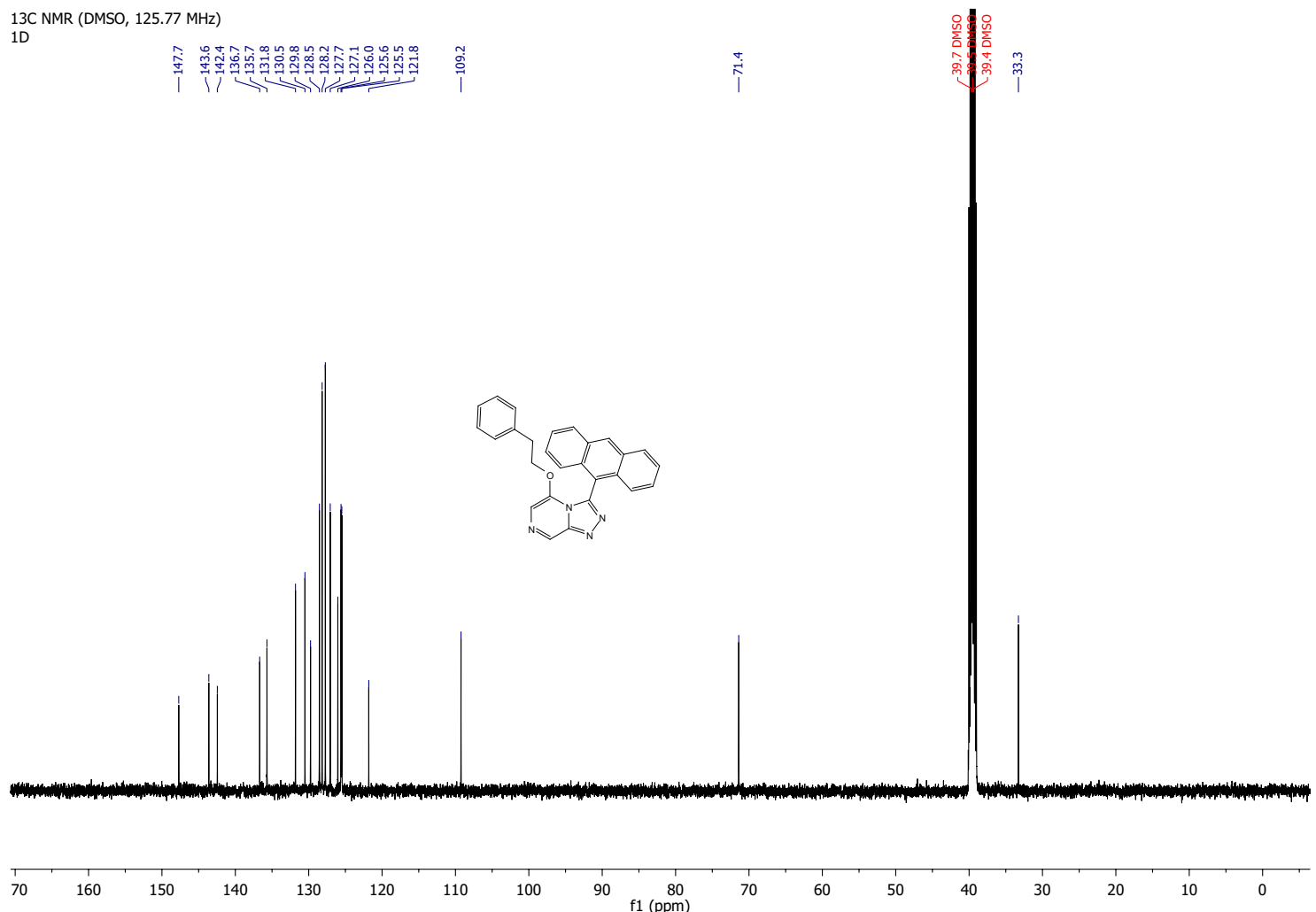
3-(4-Methoxyphenyl)-8-phenethoxy-[1,2,4] triazolo[4,3-a]pyrazine $47 \mathrm{a}$

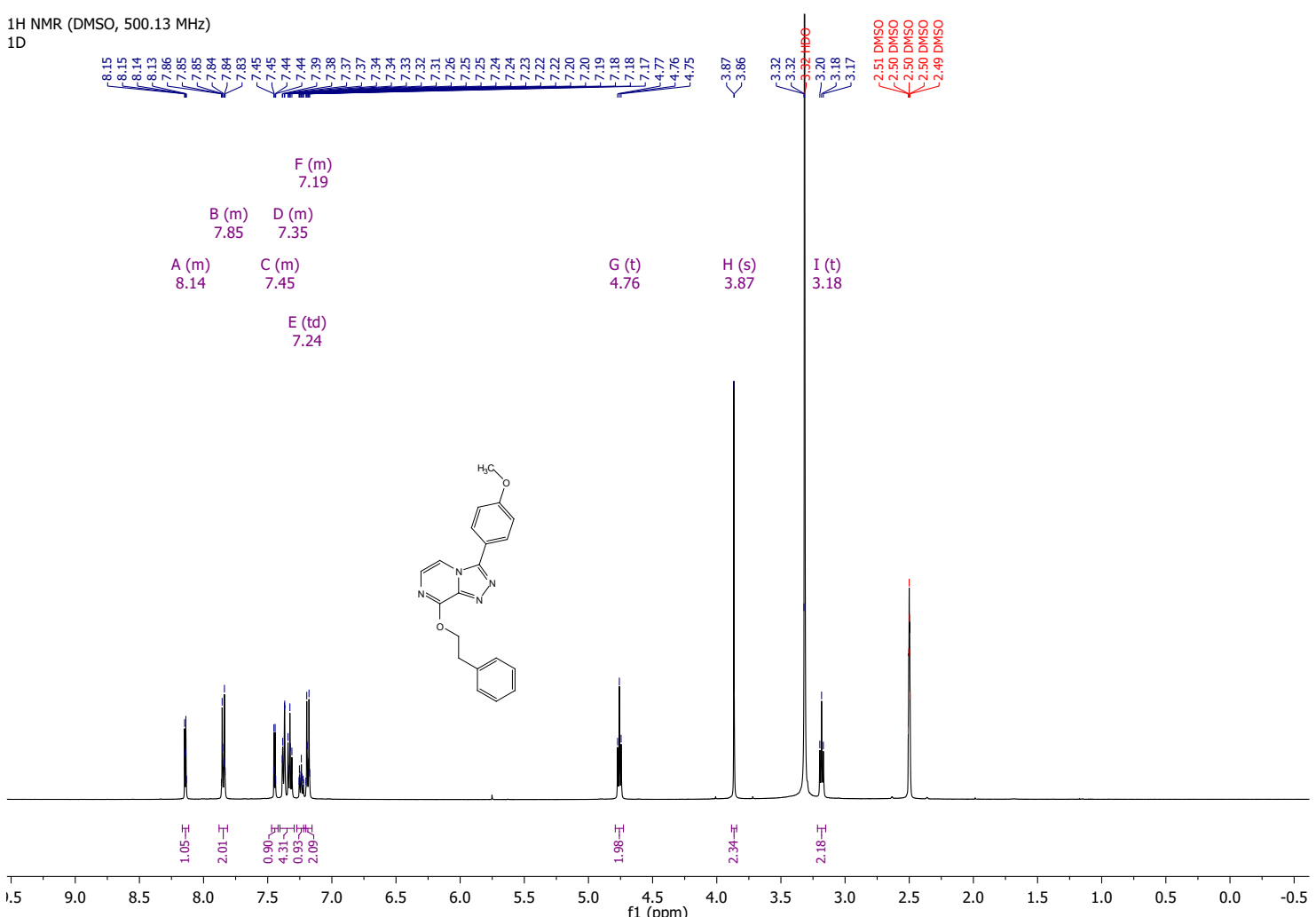

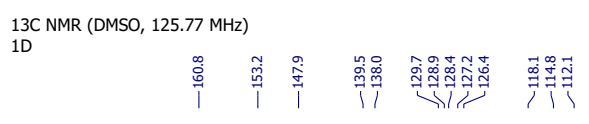
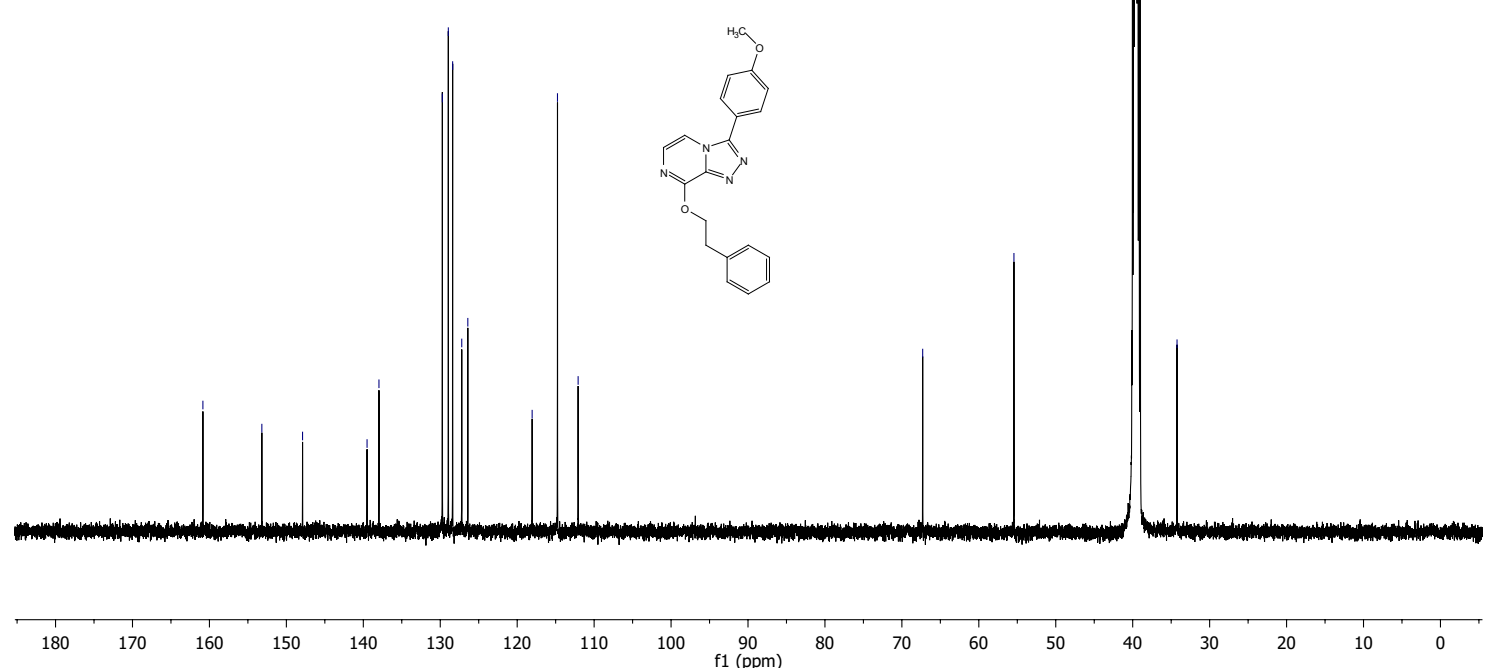
3-(4-Methoxyphenyl)-8-(phenethylthio)-[1,2,4] triazolo[4,3-a]pyrazine 47b

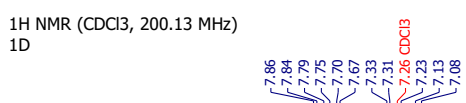

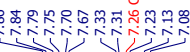

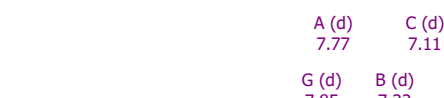

$\begin{array}{ll}7.85 & 7.32\end{array}$

I (d)
7.69
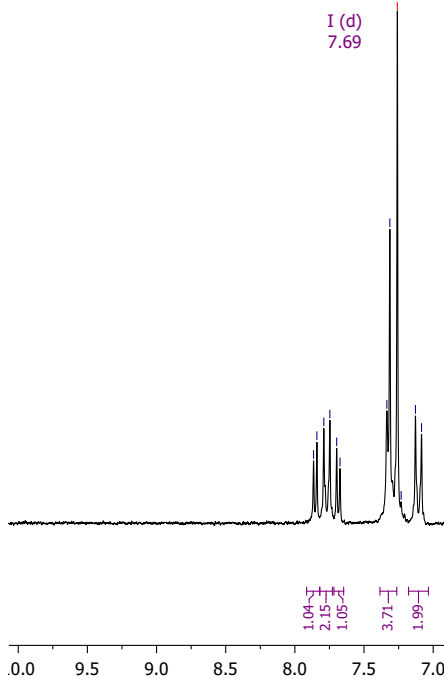

13C NMR (DMSO, $125.77 \mathrm{MHz}$ )
$1 \mathrm{D}$

$$
\text { | }
$$

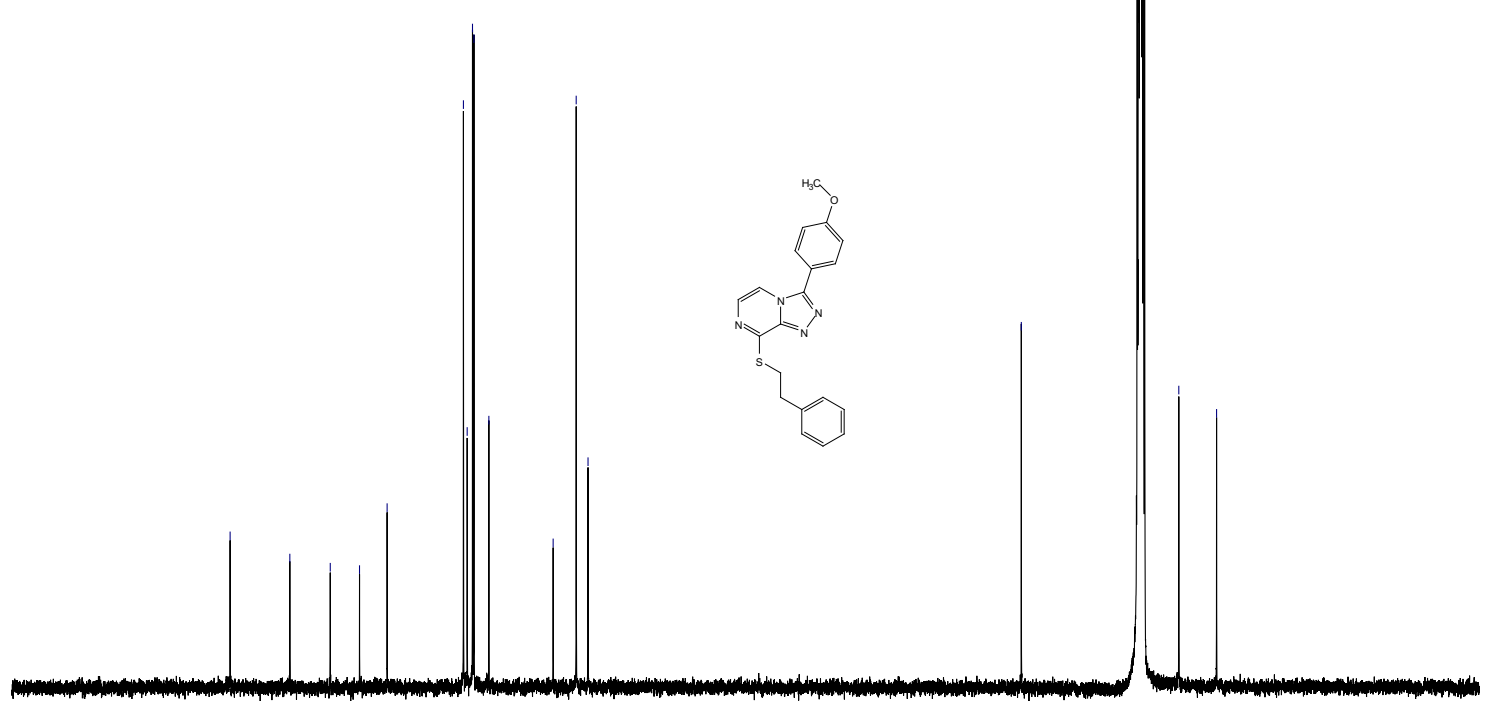


3-(4-Methoxyphenyl)- $N$-phenethyl-[1,2,4]triazolo[4,3-a]pyrazin-8-amine $47 \mathrm{c}$

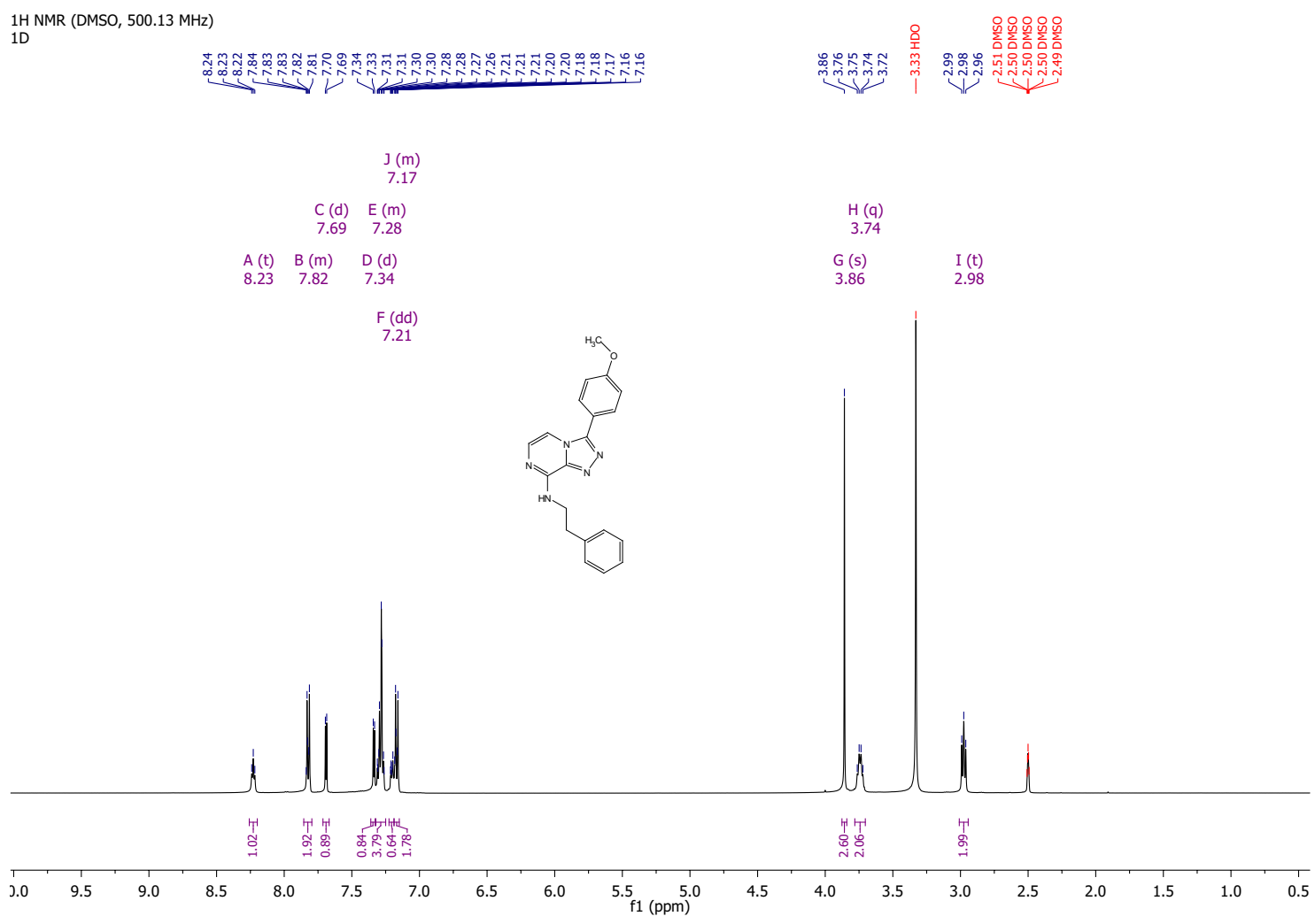

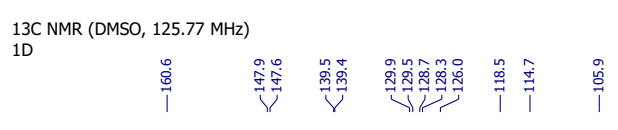

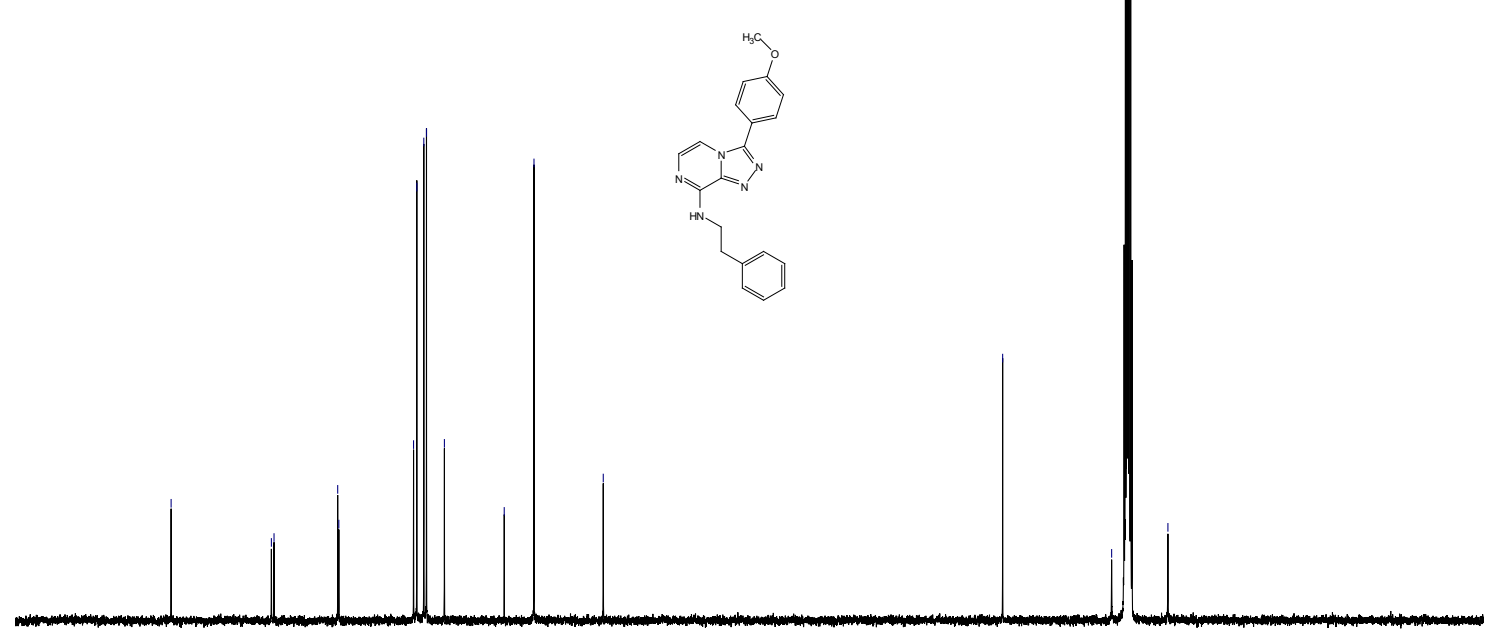

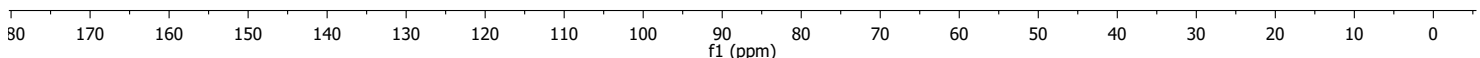


3-(3,5-Di-tert-butylphenyl)-8-phenethoxy-[1,2,4]triazolo[4,3-a]pyrazine $47 \mathrm{~d}$

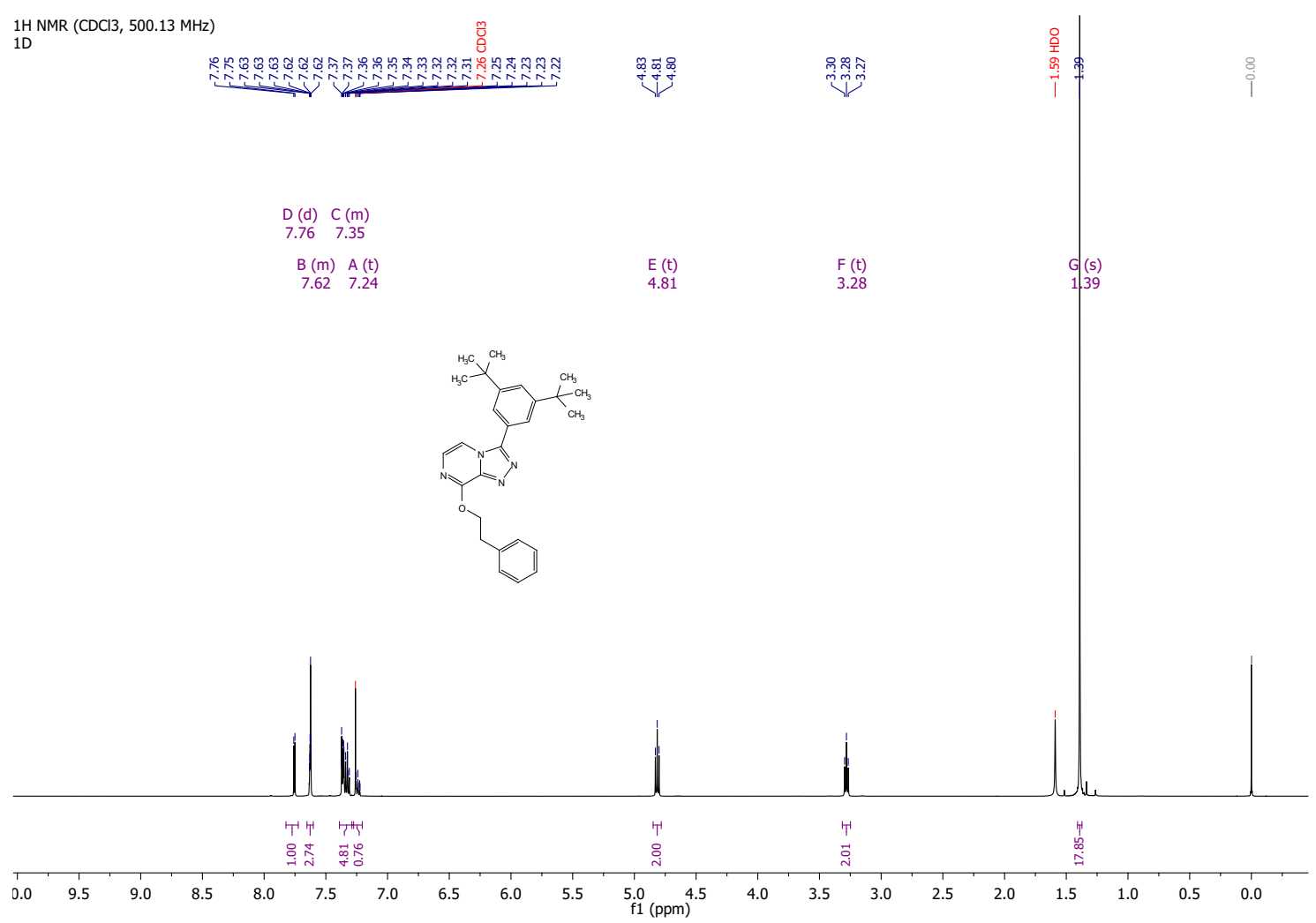

13C NMR (CDCl3, $125.77 \mathrm{MHz})$
$1 \mathrm{D}$

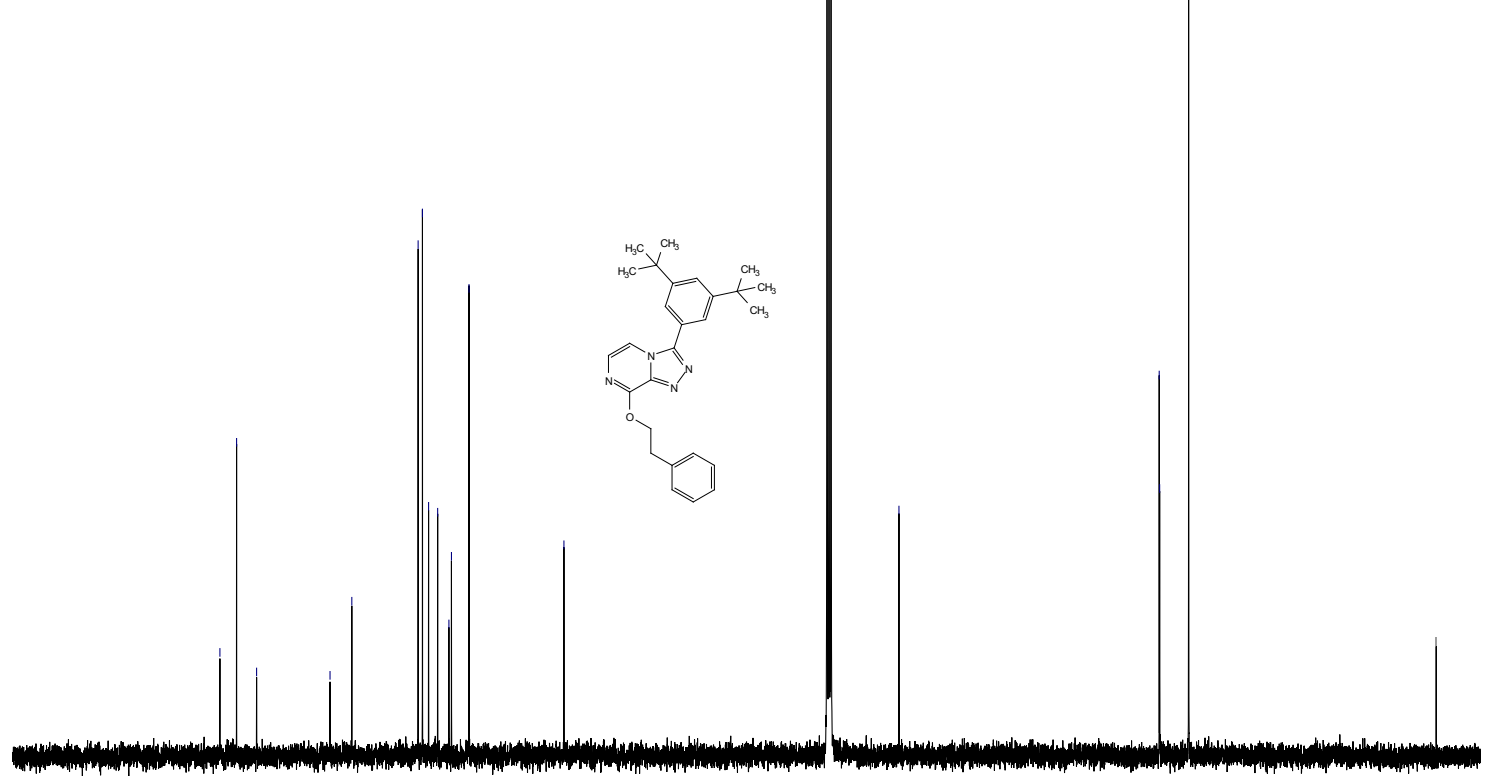

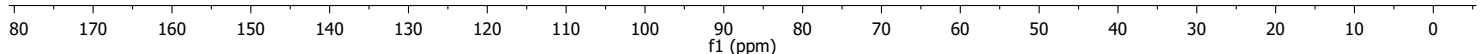


3-(Anthracen-9-yl)-8-phenethoxy-[1,2,4] triazolo[4,3-a]pyrazine $47 \mathrm{e}$

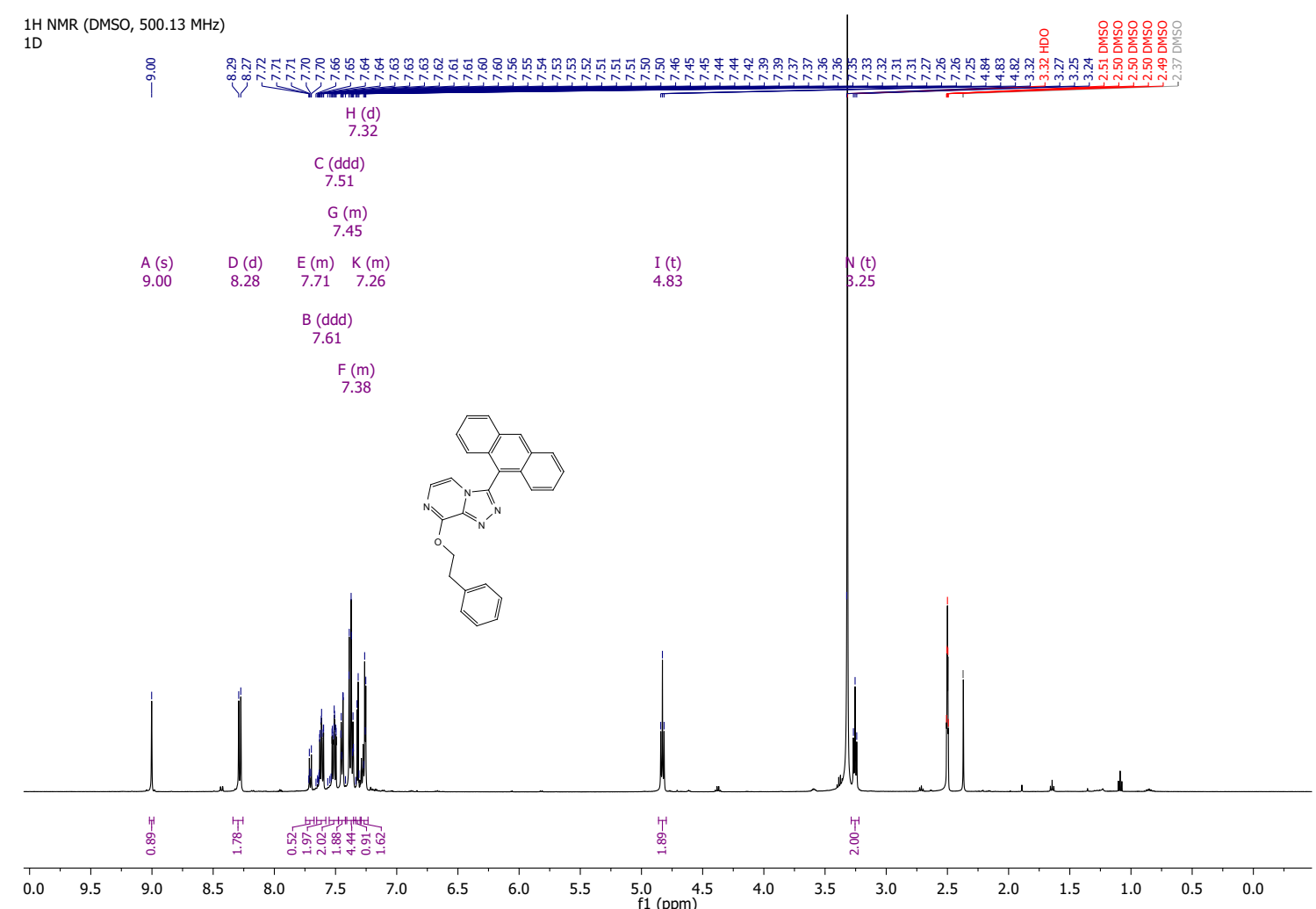

13C NMR (DMSO, $125.77 \mathrm{MHz})$

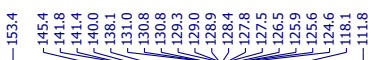

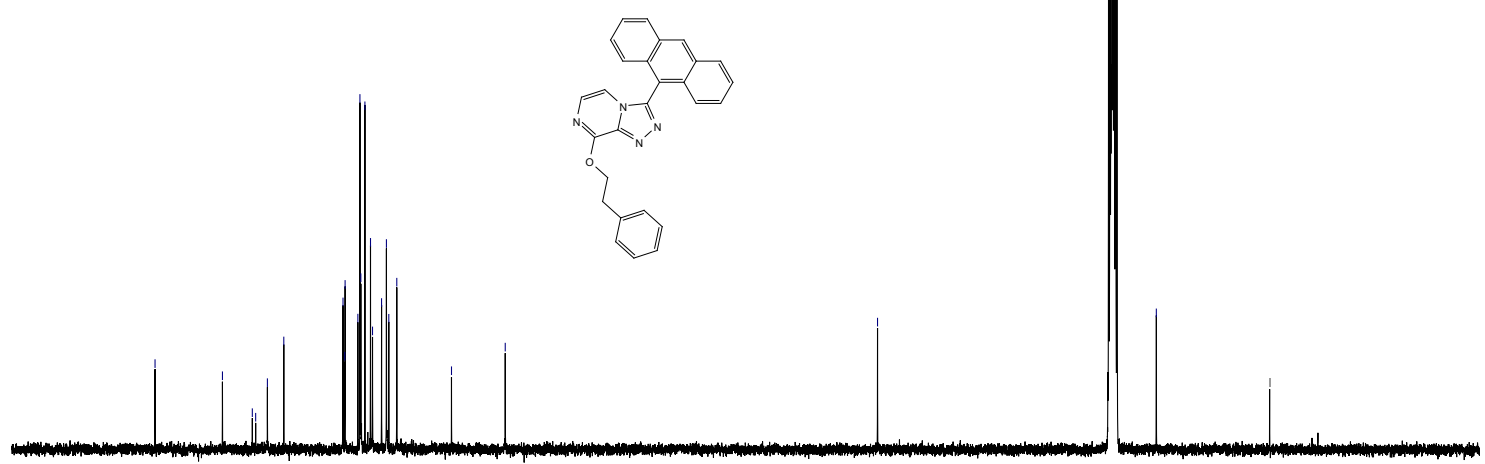

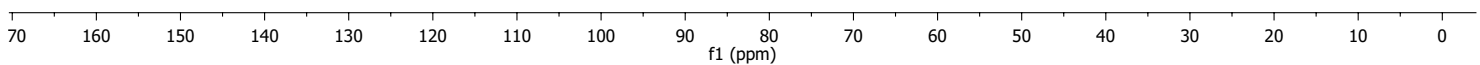


3-(2-Methoxyphenyl)-8-phenethoxy-[1,2,4]triazolo[4,3-a]pyrazine $47 \mathrm{f}$

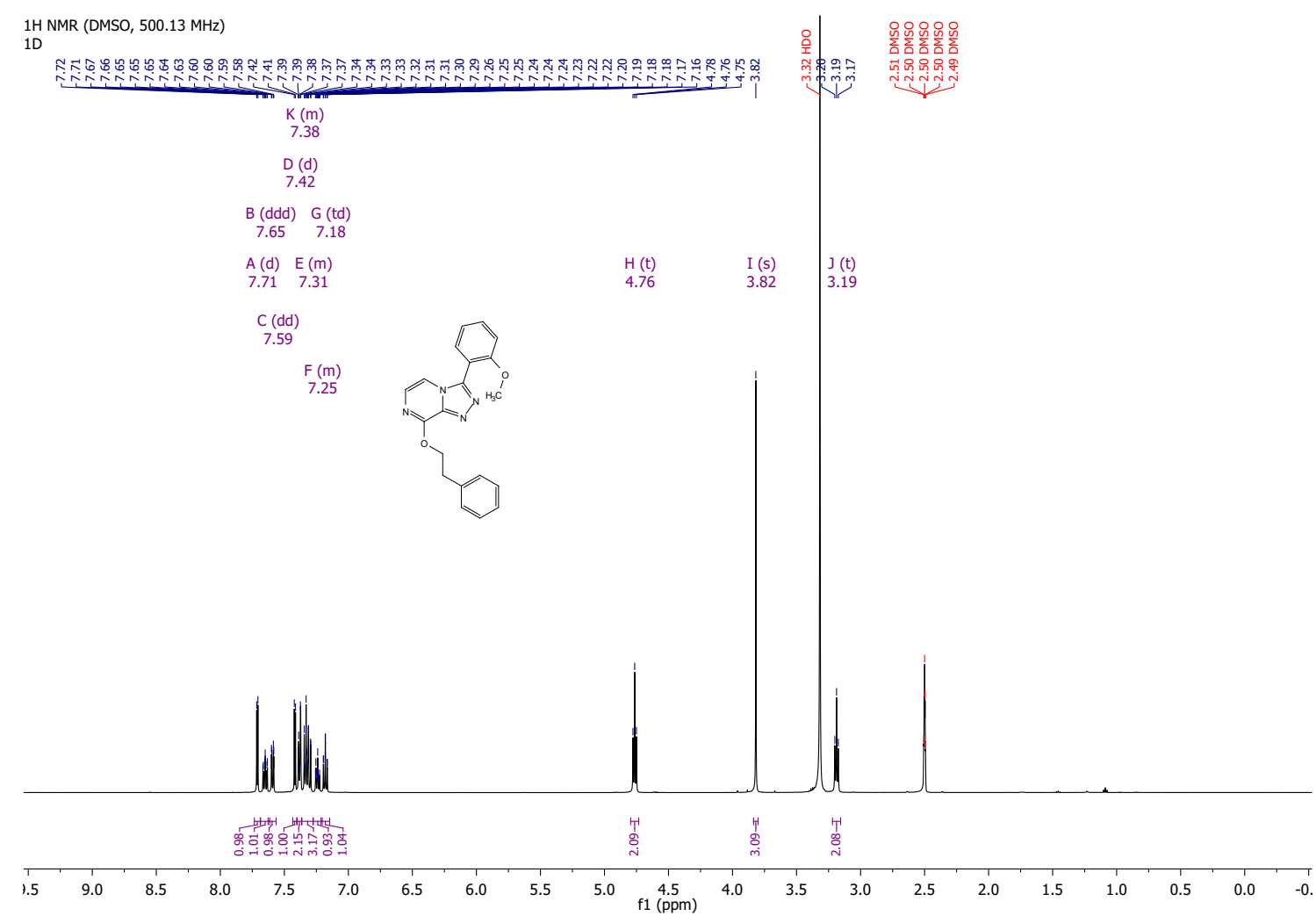

13C NMR (DMSO, $125.77 \mathrm{MHz}$ )
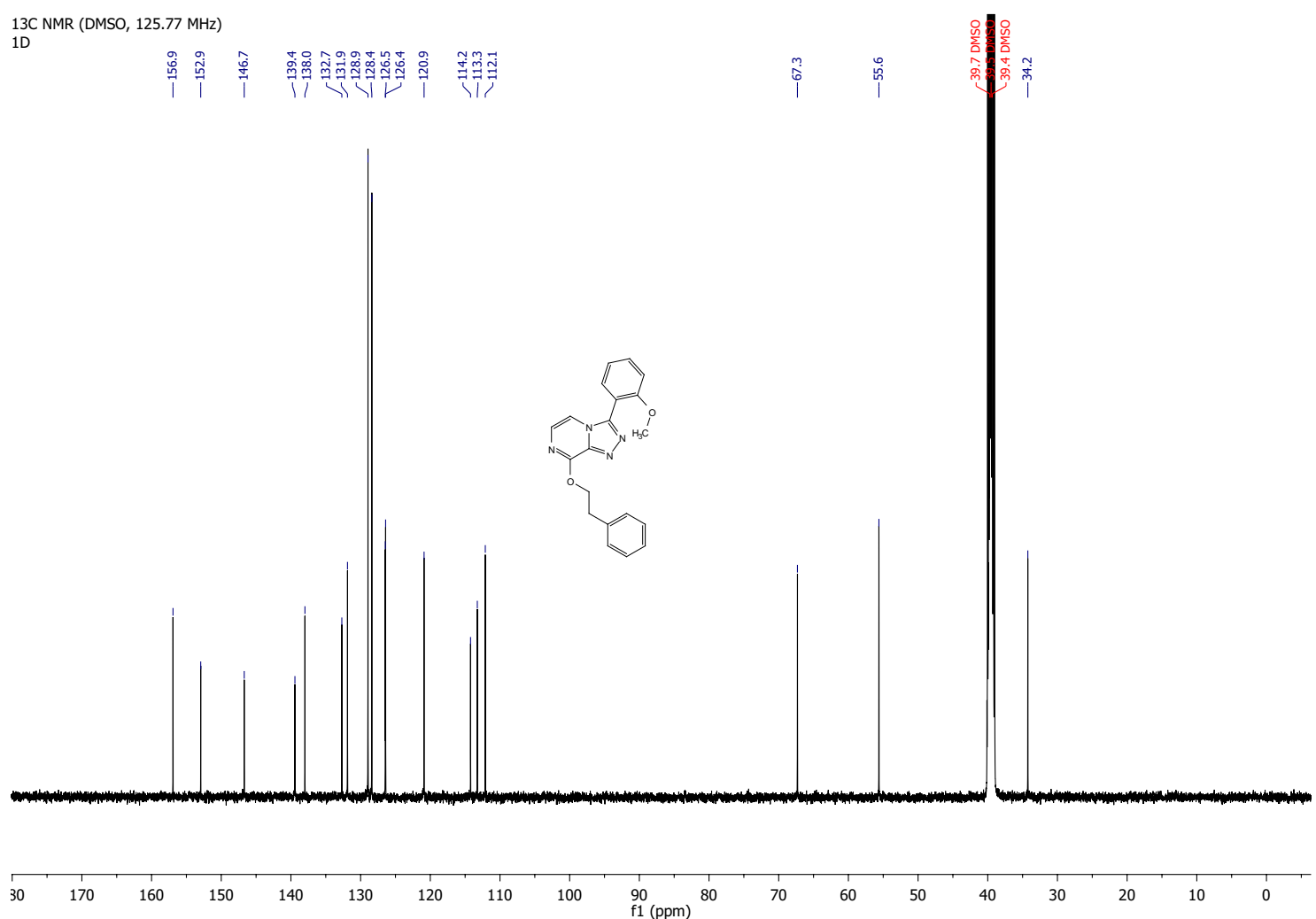
3-(4-Methoxyphenyl)-[1,2,4] triazolo[4,3-a]pyrazin-8-ol 48a

1H NMR (DMSO, $500.13 \mathrm{MHz}$ )

1D

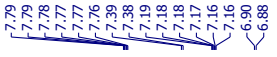
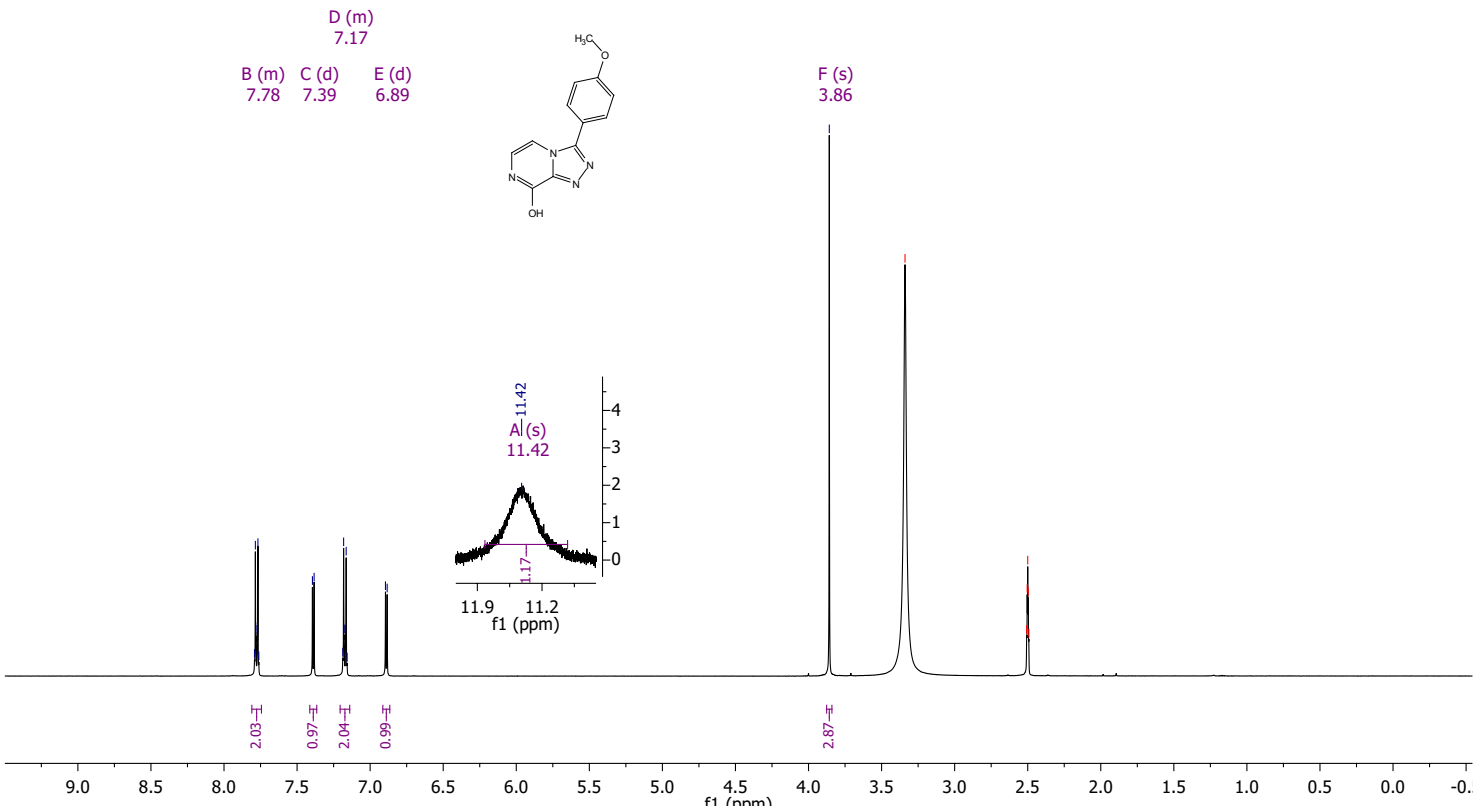

13C NMR (DMSO, $125.77 \mathrm{MHz}$ )

।
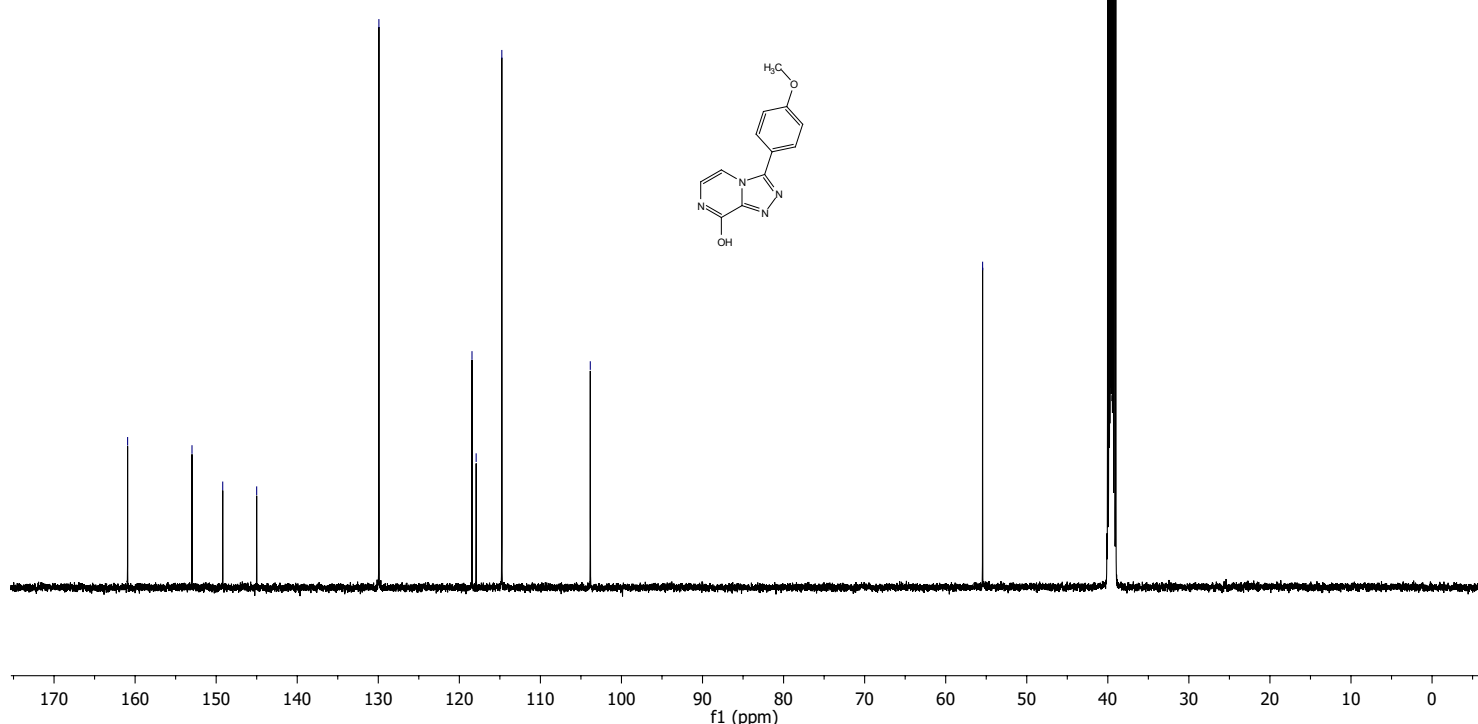
3-(4-Nitrophenyl)-[1,2,4] triazolo[4,3-a]pyrazin-8-ol 48b

1H NMR (DMSO, $500.13 \mathrm{MHz}$ )
$1 \mathrm{D}$
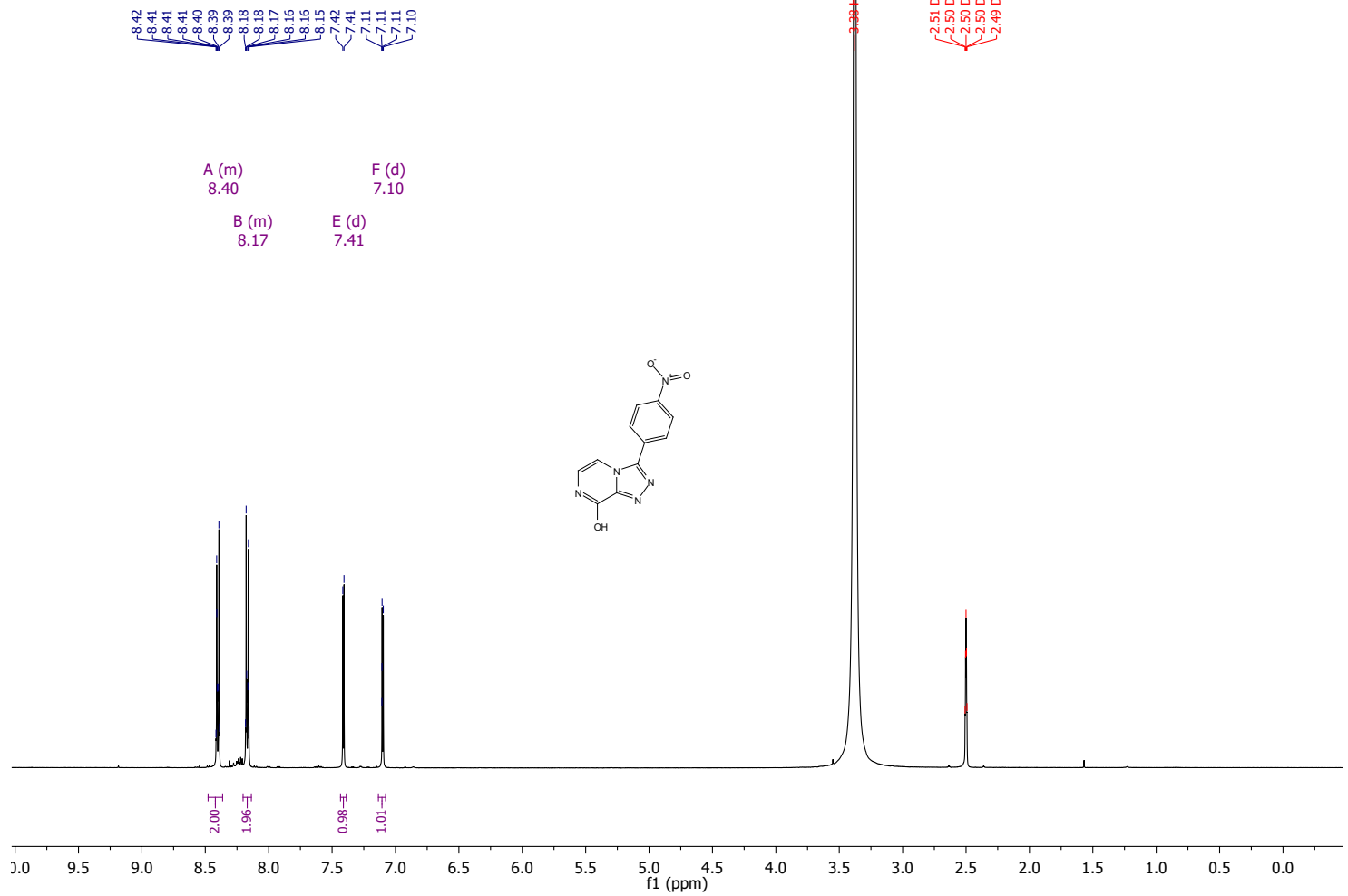

$13 C$ NMR (DMSO, $125.77 \mathrm{MHz})$
$1 \mathrm{D}$

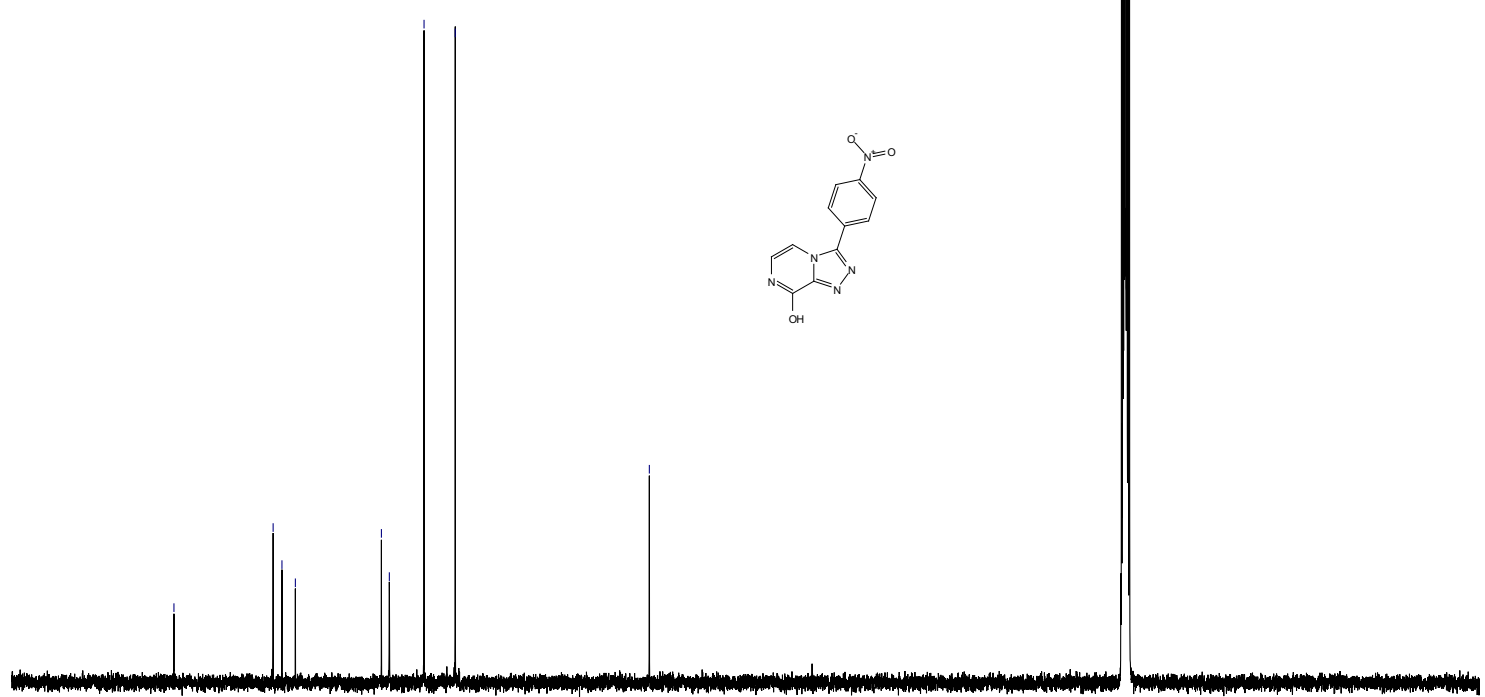

80

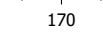

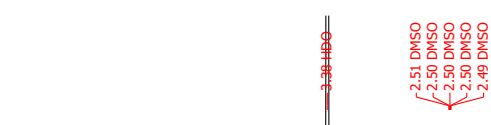

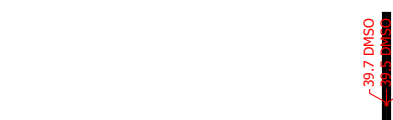

90 (ppm) 80
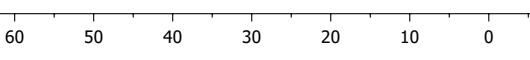

30 
3-(2-Methoxyphenyl)-[1,2,4]triazolo[4,3-a]pyrazin-8-ol 48c

1H NMR (DMSO, $500.13 \mathrm{MHz})$

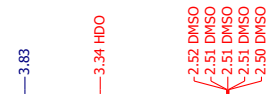

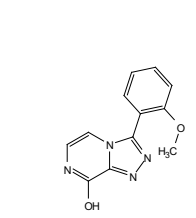

$\begin{array}{cc}7.56 & 7.00 \\ D \text { (ddd) } & \text { I (td) } \\ 7.65 & 7.17 \\ & \\ \text { G (dd) } & \text { L (d) } \\ 7.29 & 6.86\end{array}$
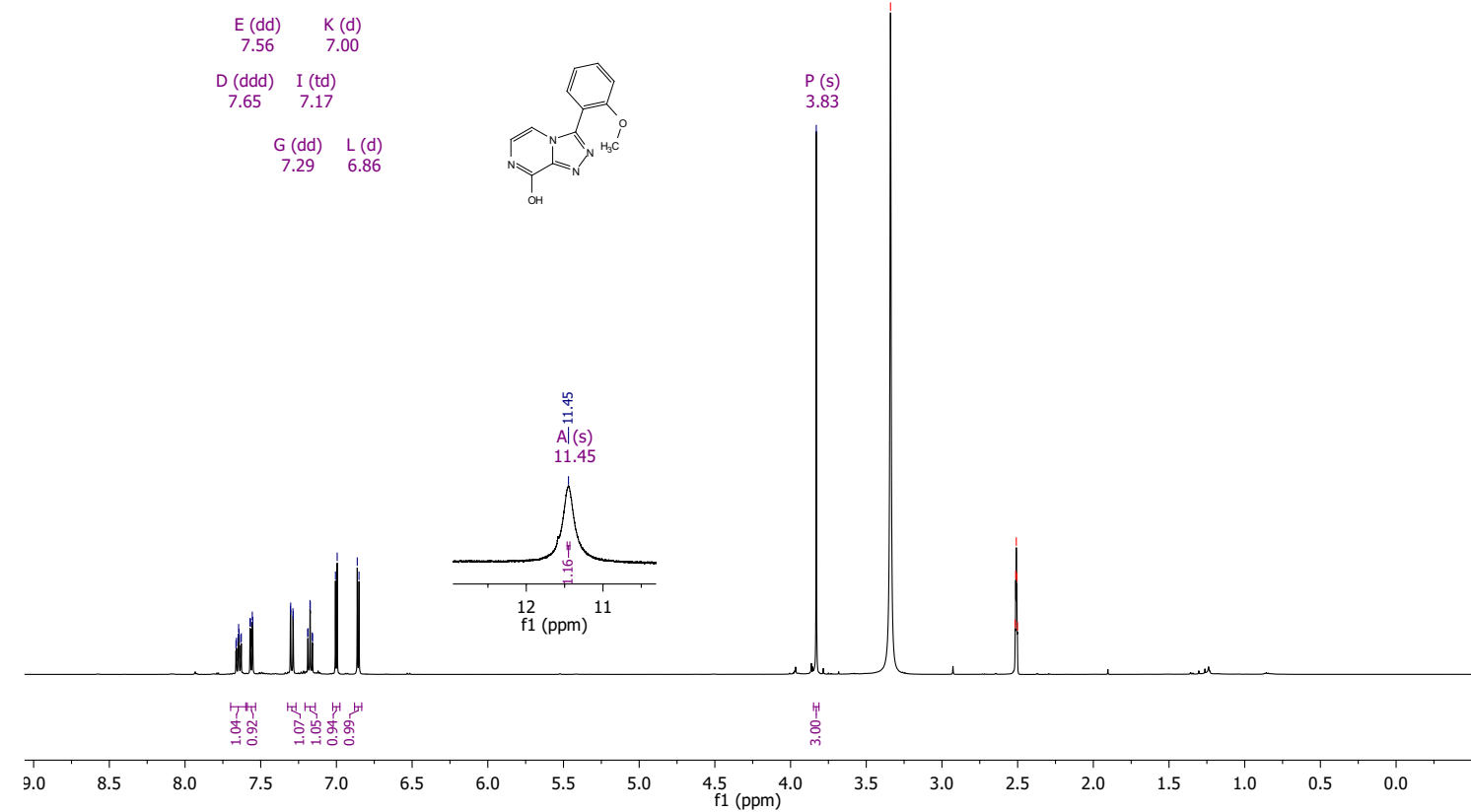

13C NMR (DMSO, $125.77 \mathrm{MHz}$ )
1D

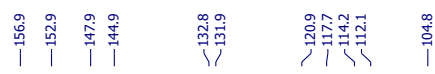

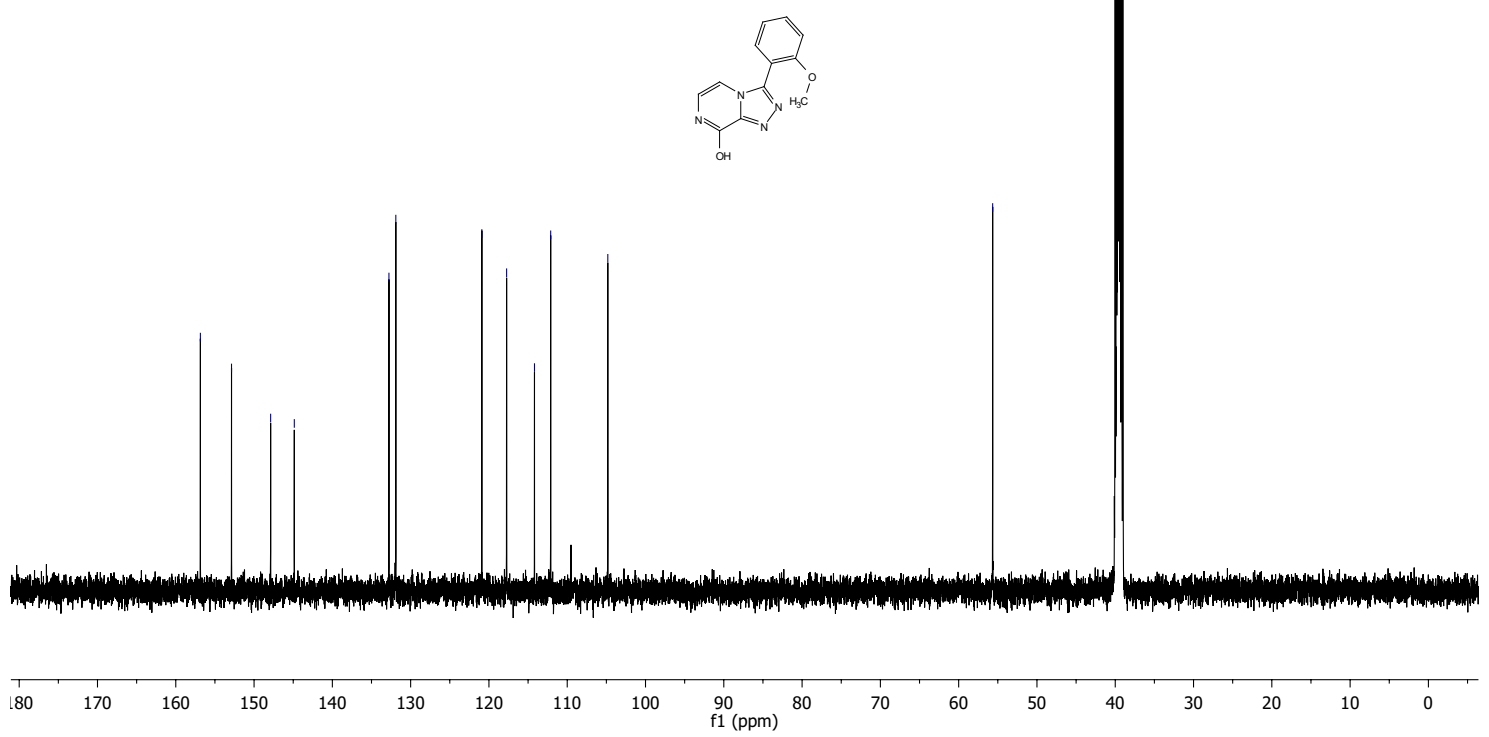


3-(2-Nitrophenyl)-[1,2,4] triazolo[4,3-a]pyrazin-8-ol 48d

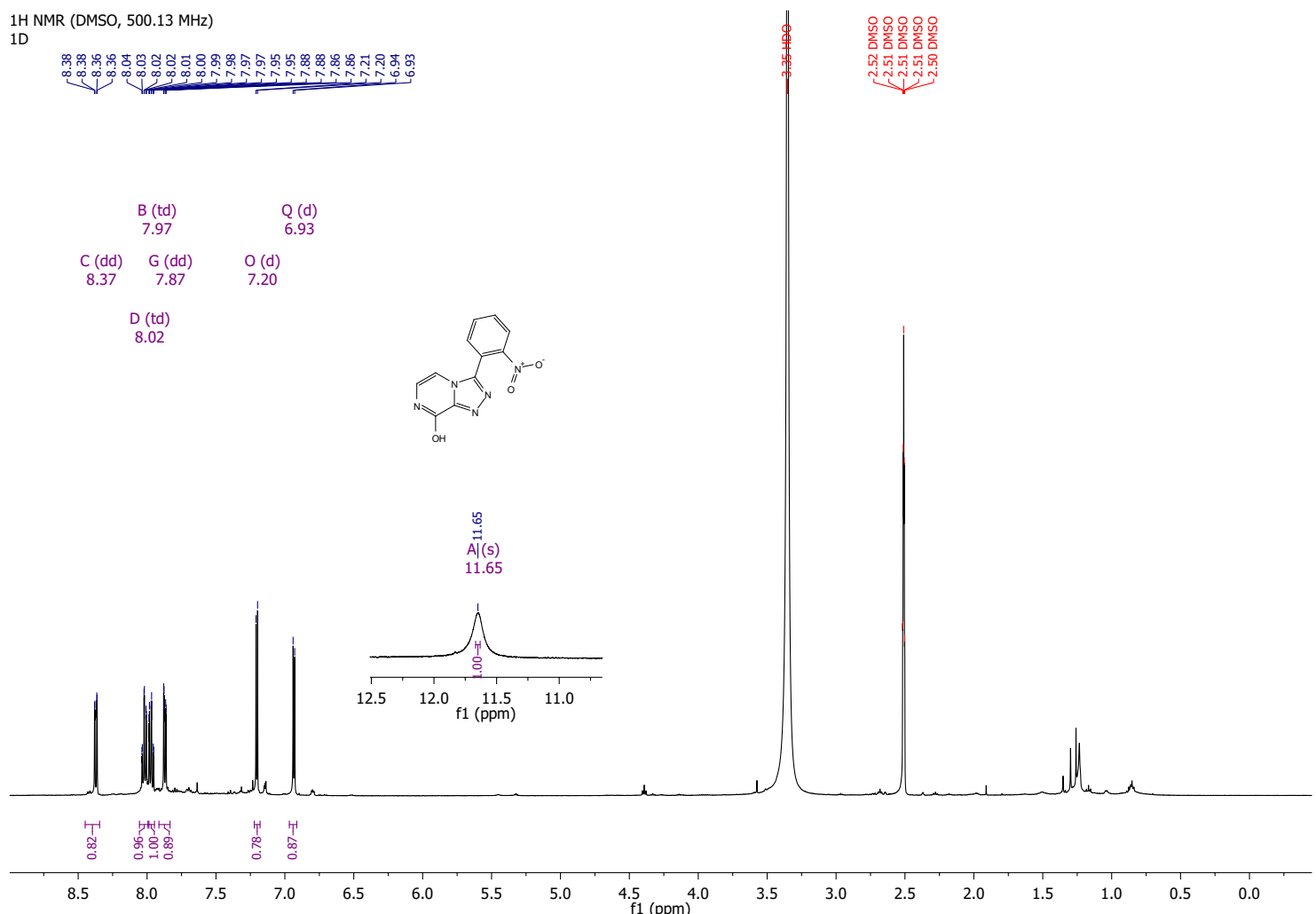

13C NMR (DMSO, 125.77 MHz)
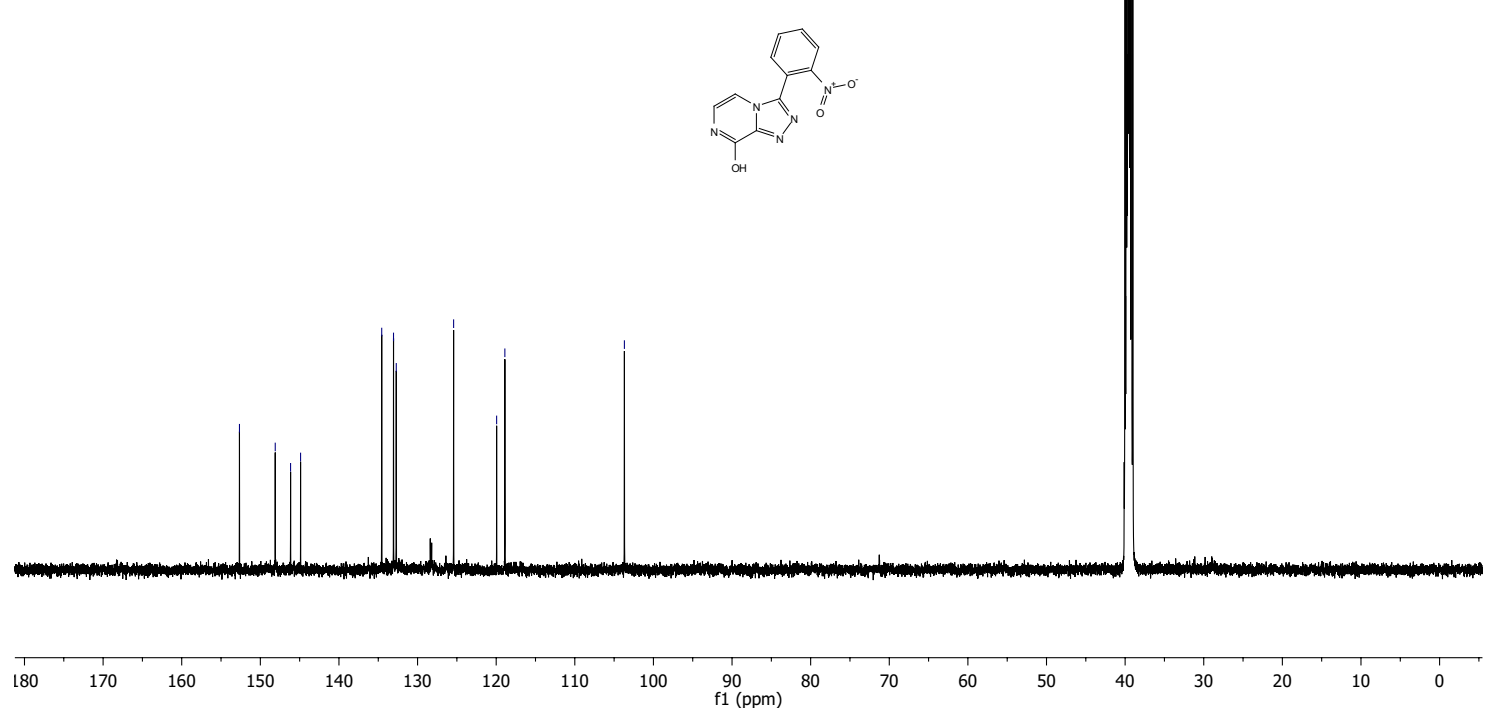
3-(4-Methoxyphenyl)-[1,2,4] triazolo[4,3-a]pyrazine 49

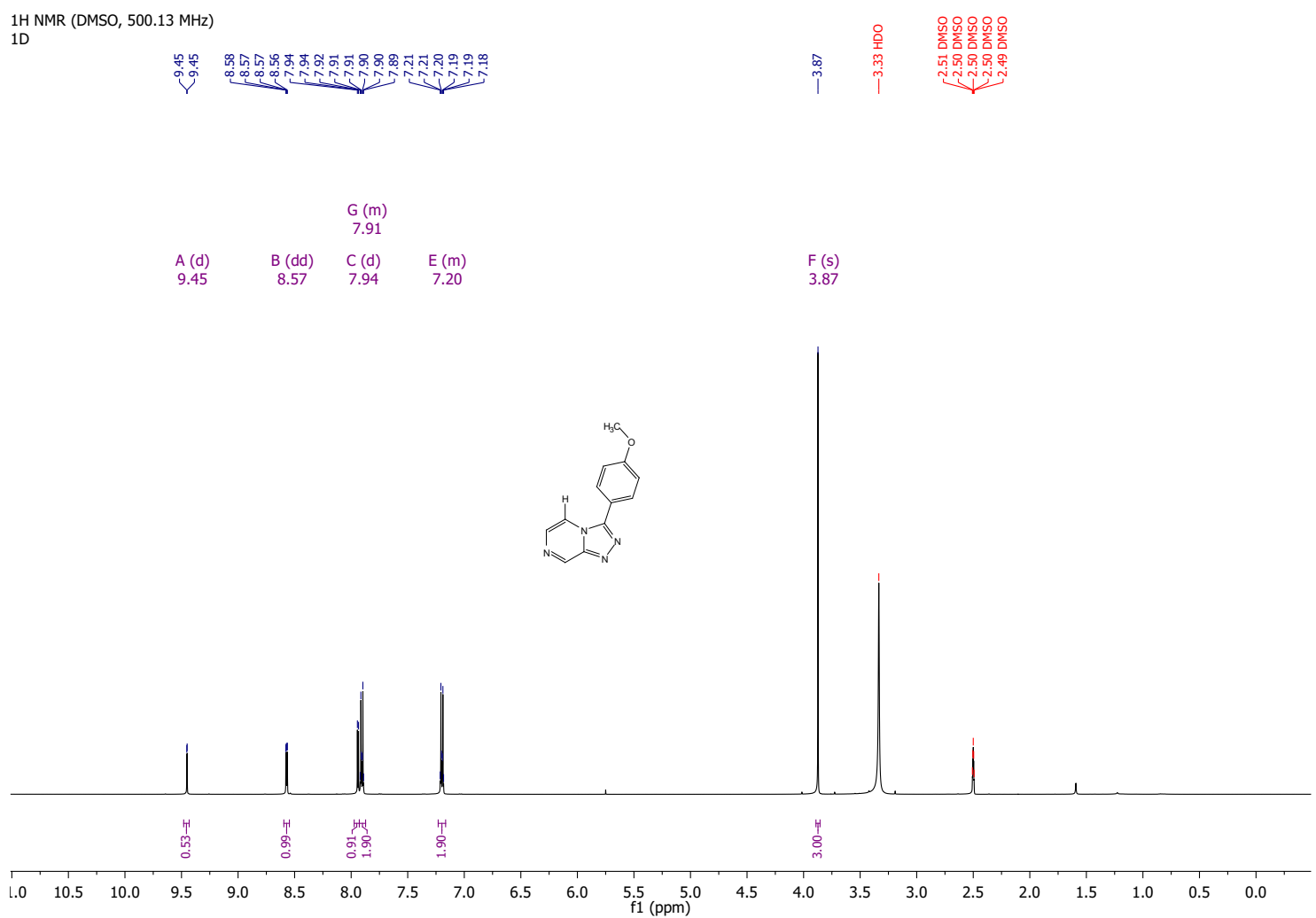

13C NMR (DMSO, $125.77 \mathrm{MHz})$
DD

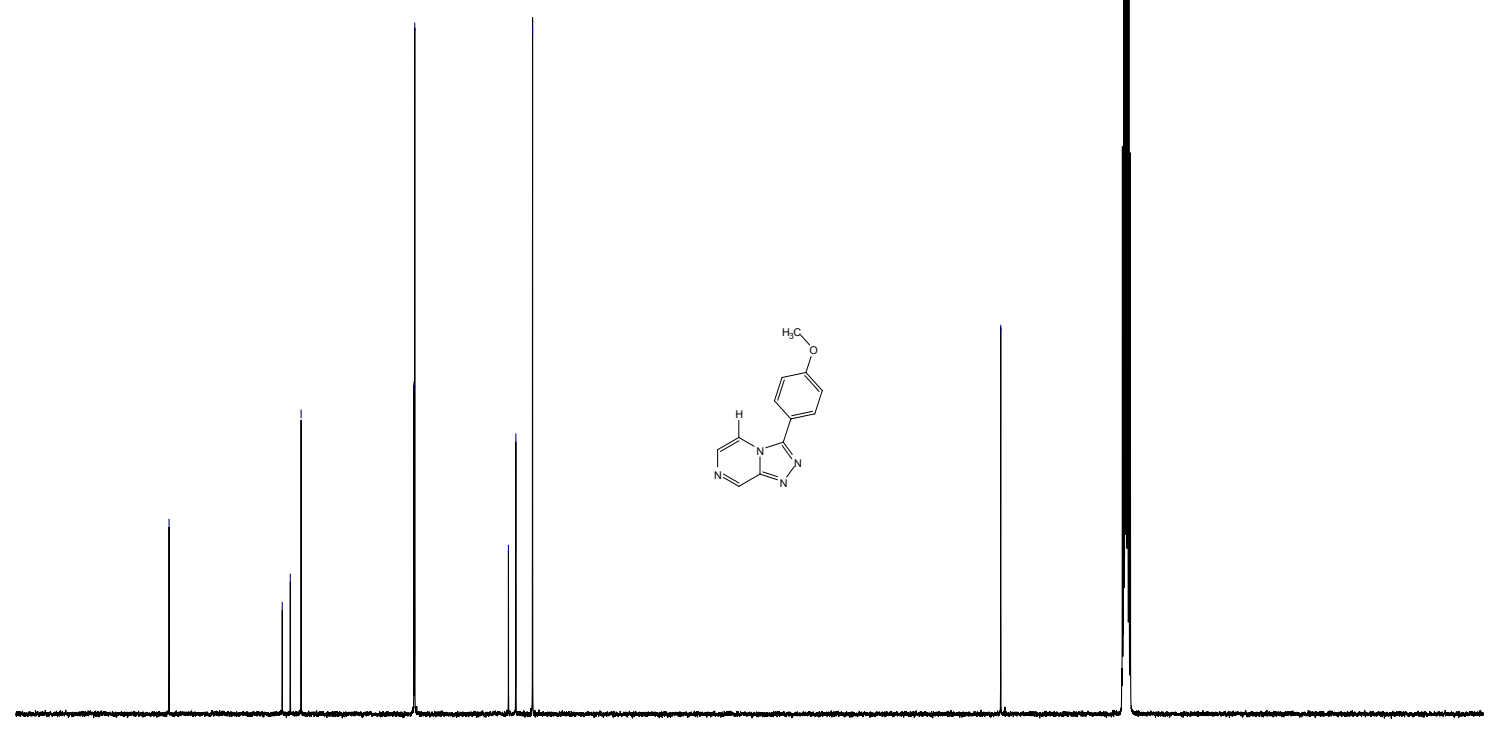

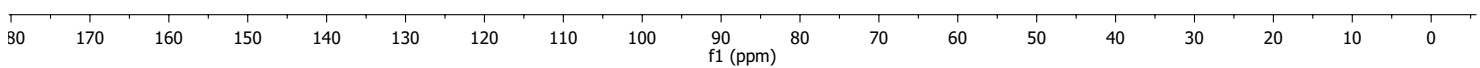


3-(4-Methoxyphenyl)-5-(1-phenethyl-1H-imidazol-2-yl)-4H-1,2,4-triazole 50
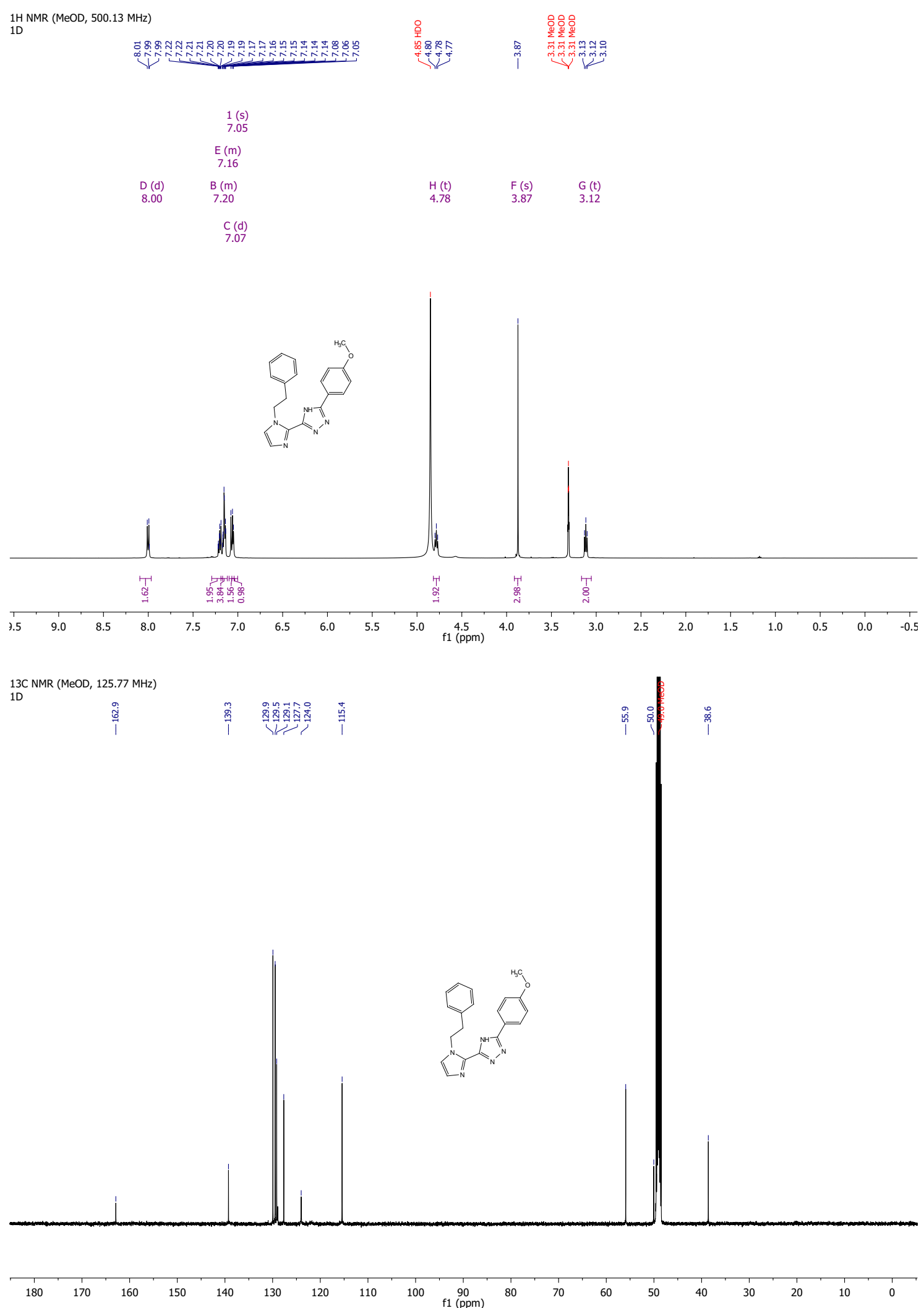
5-(Piperidin-1-yl)-[1,2,4]triazolo[4,3-a]pyrazine 55

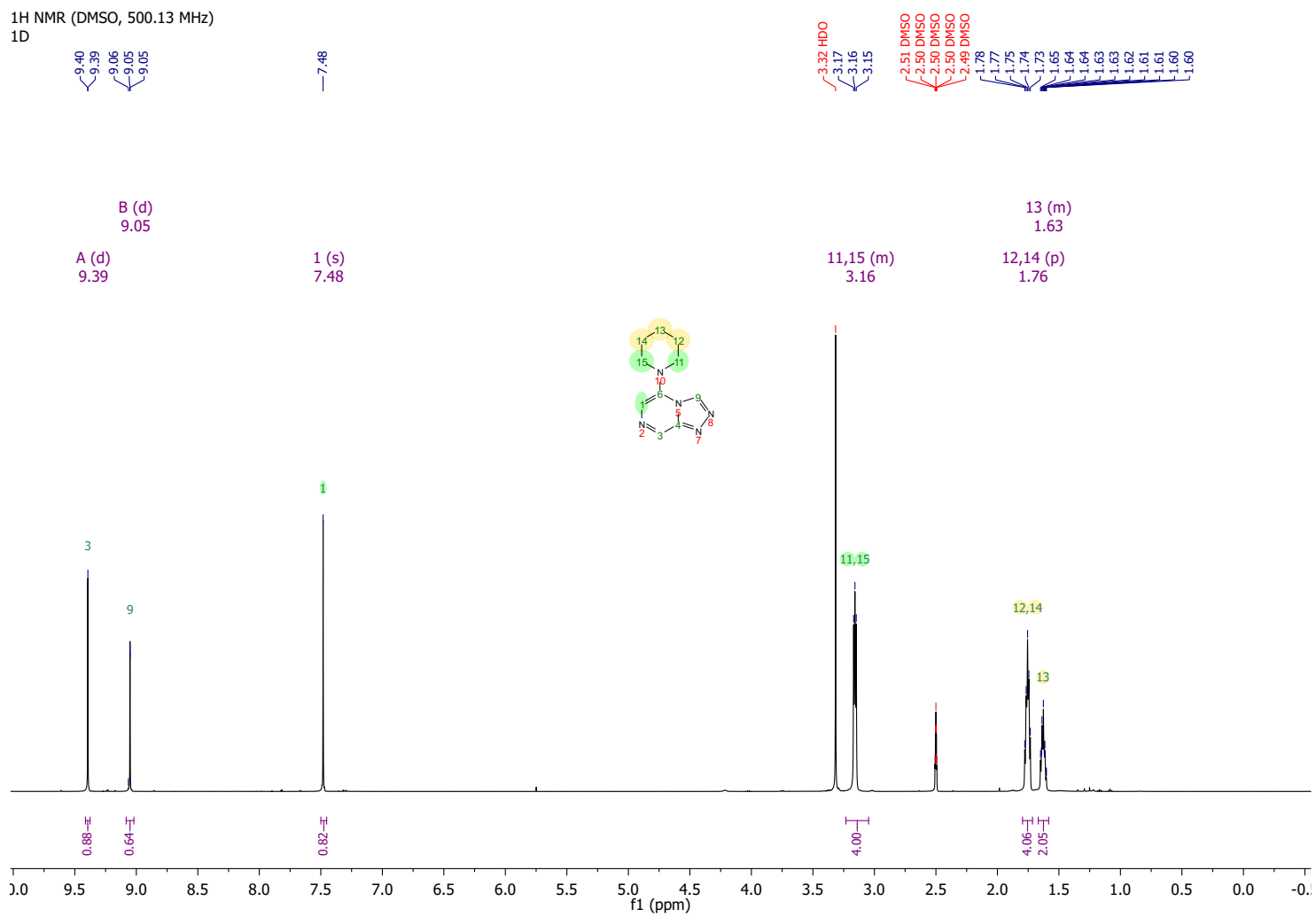

$13 \mathrm{C}$ NMR (DMSO, $125.77 \mathrm{MHz})$
$1 \mathrm{D}$

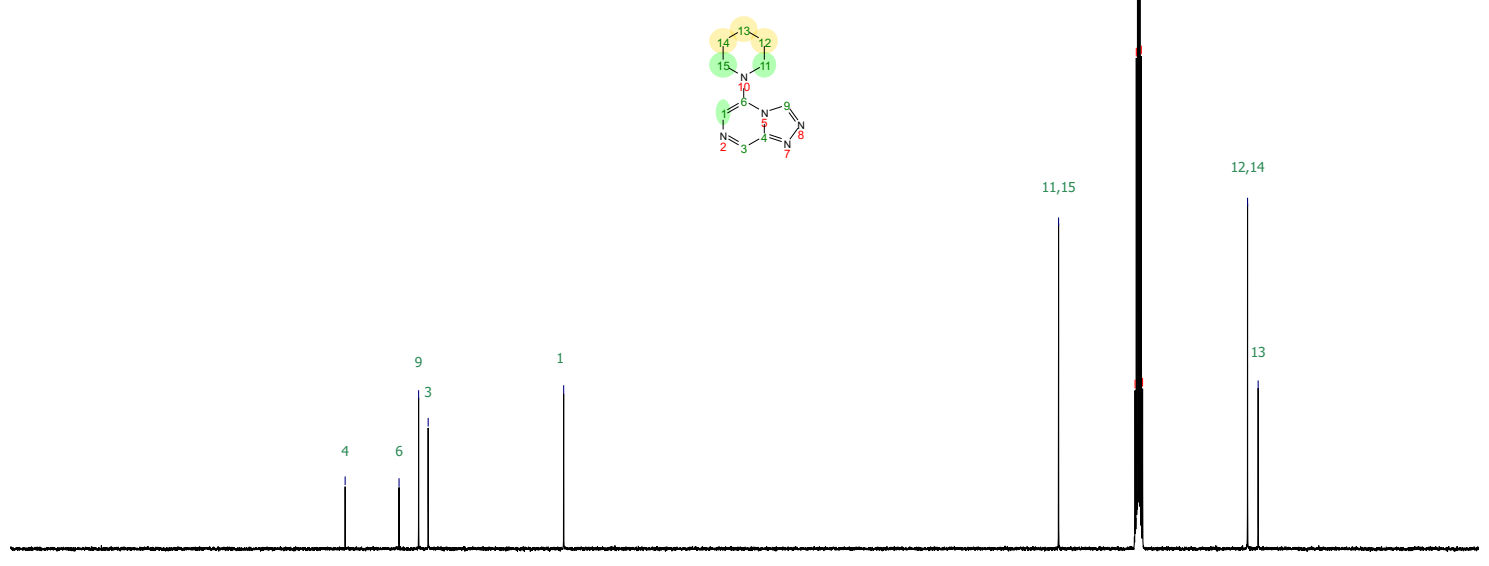

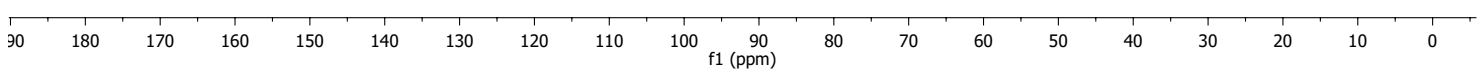


8-(Piperidin-1-yl)-[1,2,4] triazolo[4,3- $a]$ pyrazine 56
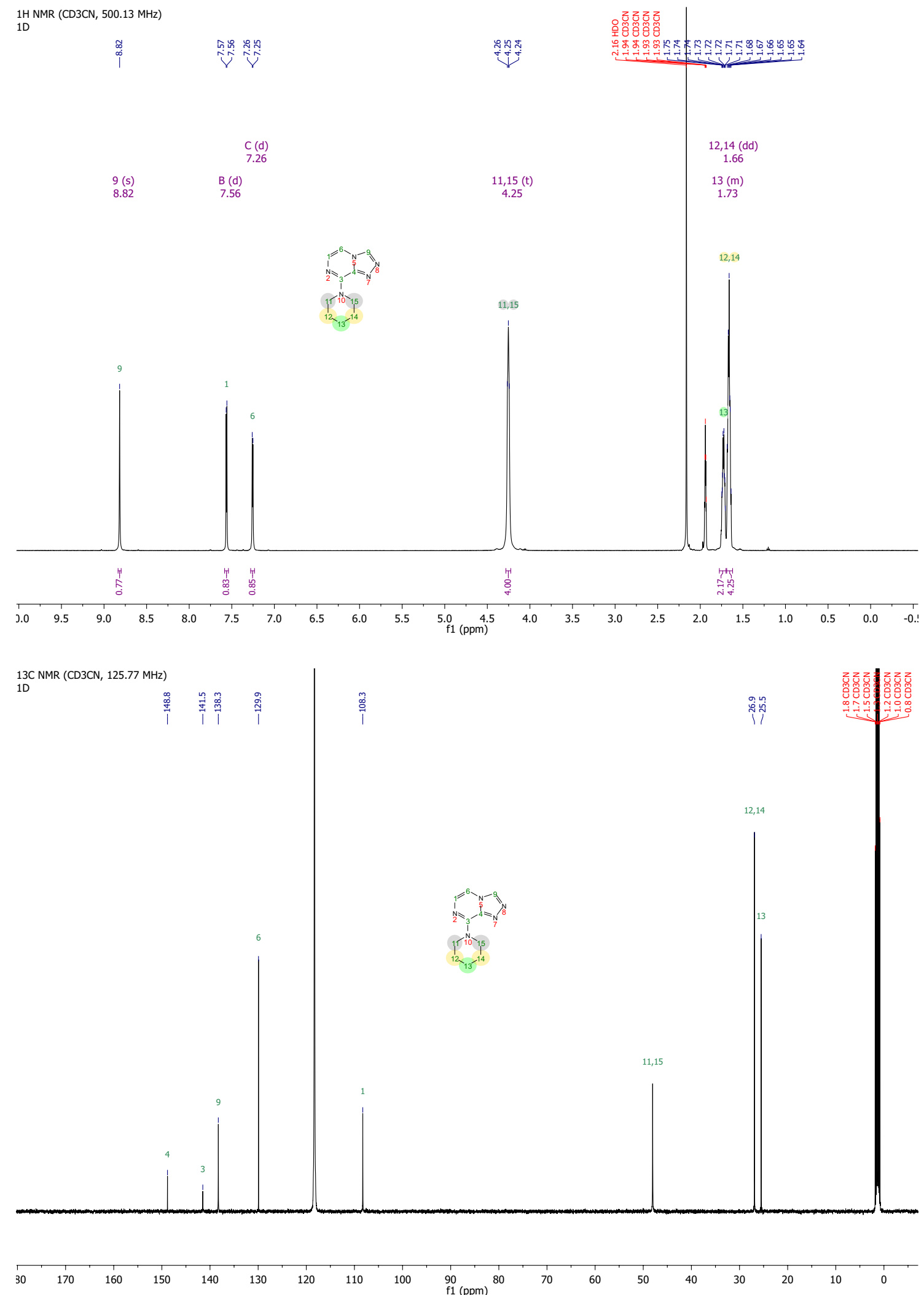
5-(Piperidin-1-yl)-[1,2,4]triazolo[4,3-a]pyrazine-3- $d 57$

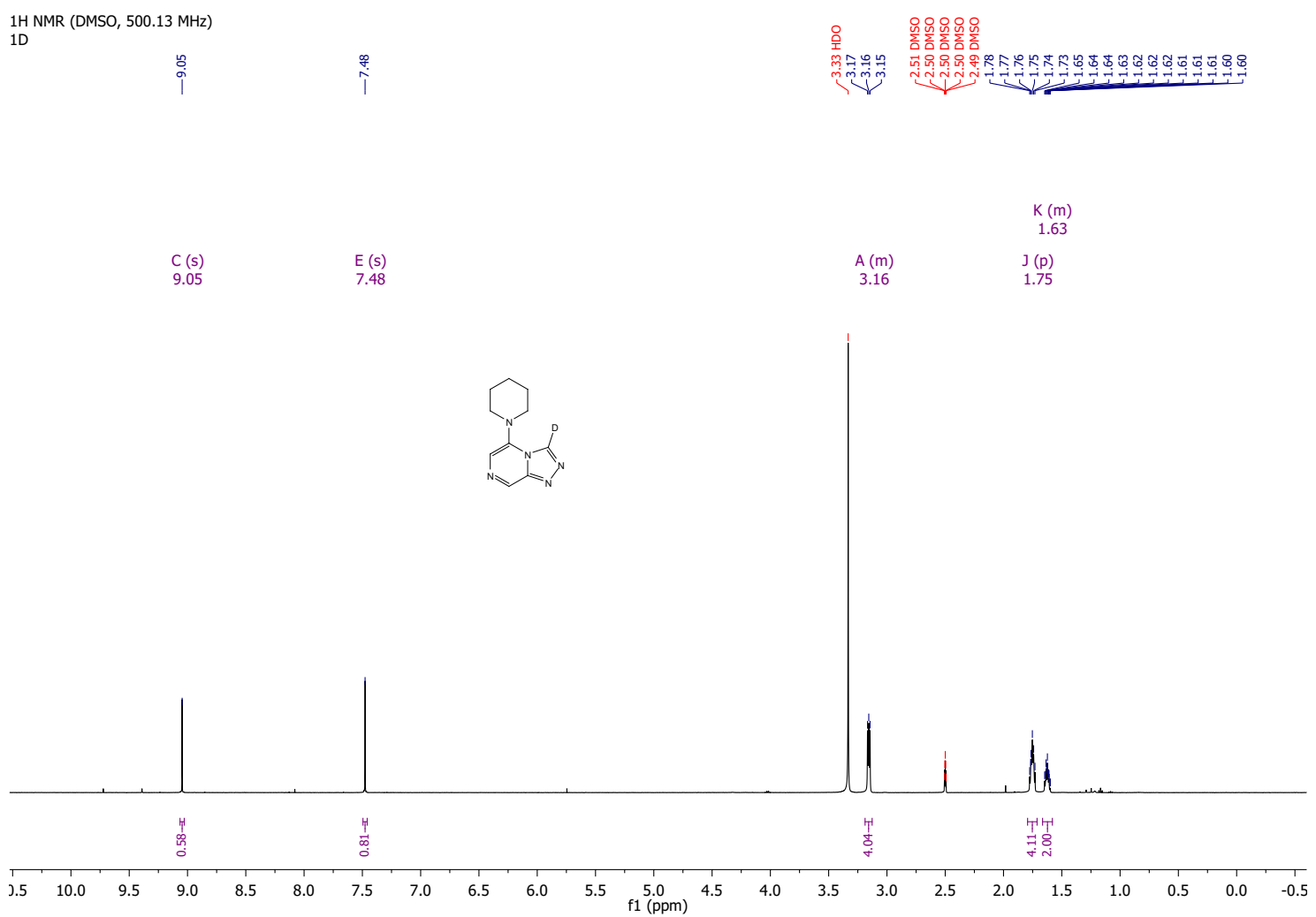

13C NMR (DMSO, $125.77 \mathrm{MHz})$
DD

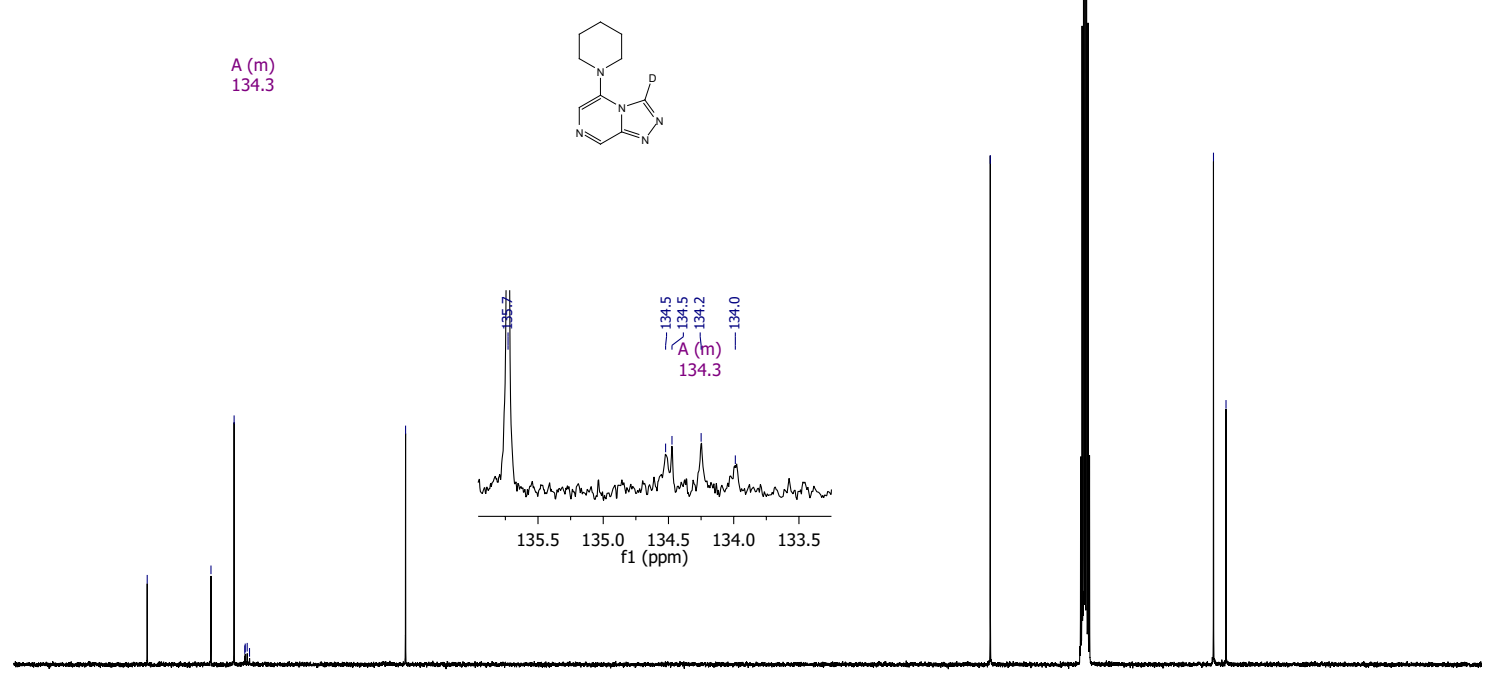

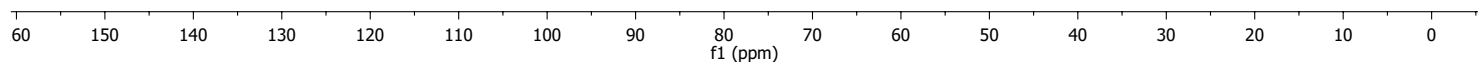




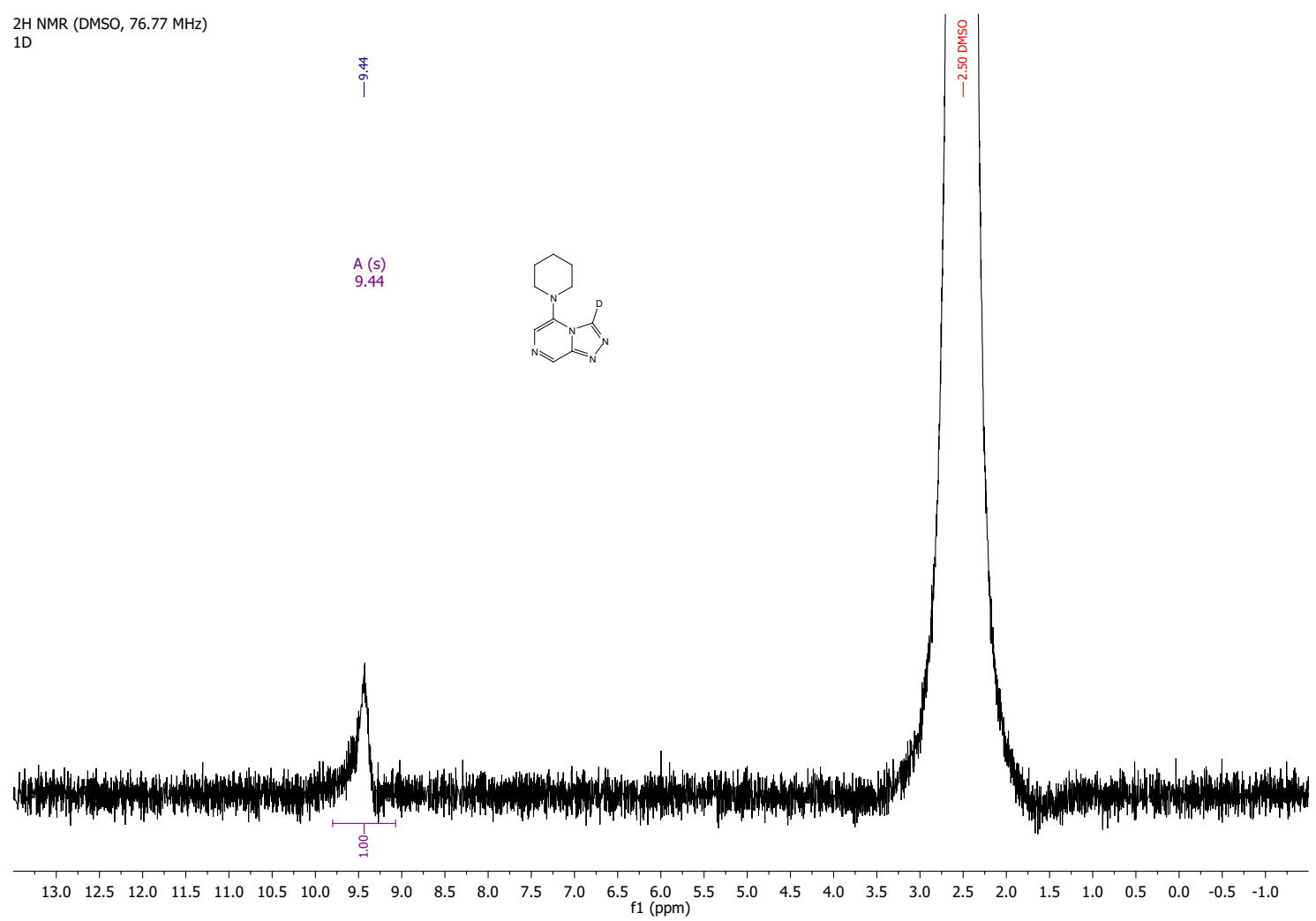


8-(Piperidin-1-yl)-[1,2,4]triazolo[4,3-a]pyrazine-3,5- $d_{2} 58$

1H NMR (DMSO, $500.13 \mathrm{MHz}$ )

1D

$\stackrel{\vec{m}}{i}$

A (s)
7.31

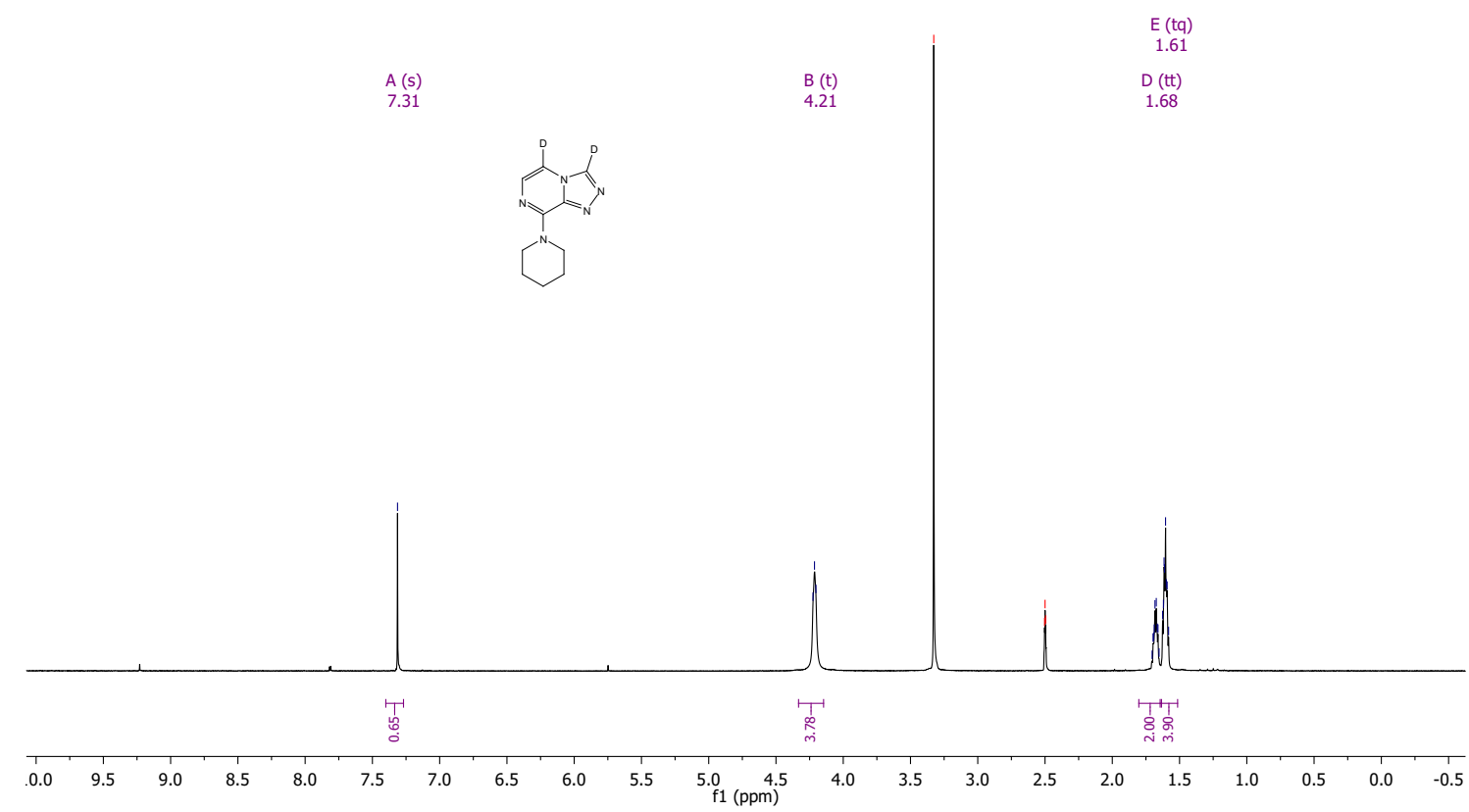

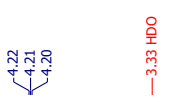

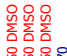

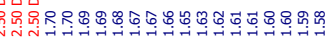

13C NMR (DMSO, $125.77 \mathrm{MHz}$ )
1D

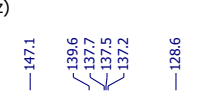

$\sqrt{1}$

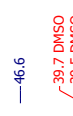

$A(m)$
137.5

$B(t)$
107.4
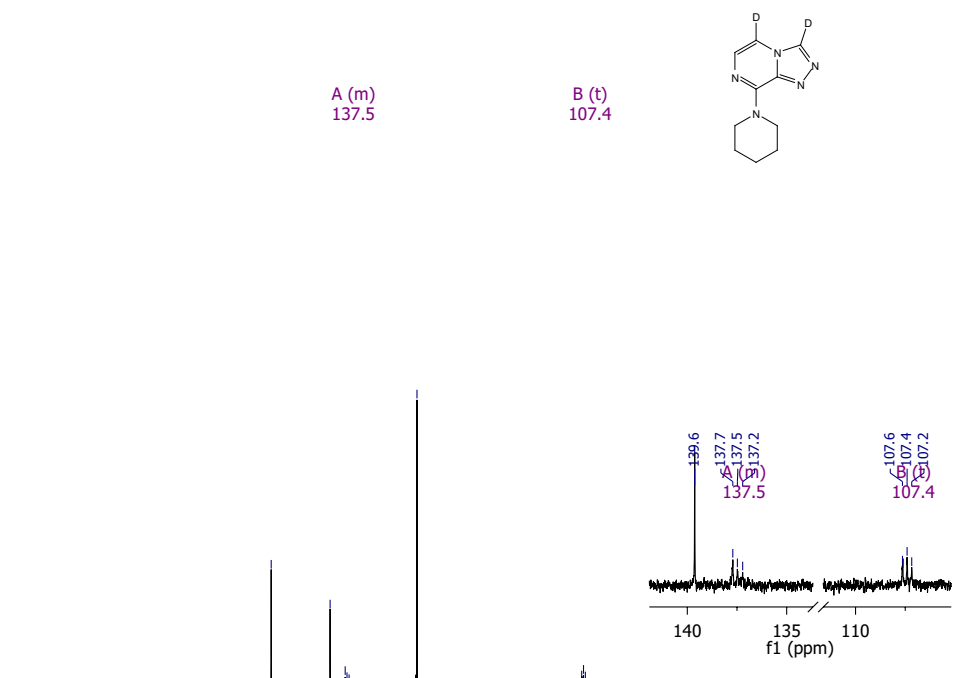

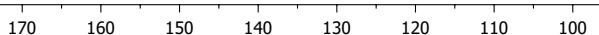

${ }_{\mathrm{f} 1}^{90}(\mathrm{ppm}){ }^{80}$ 


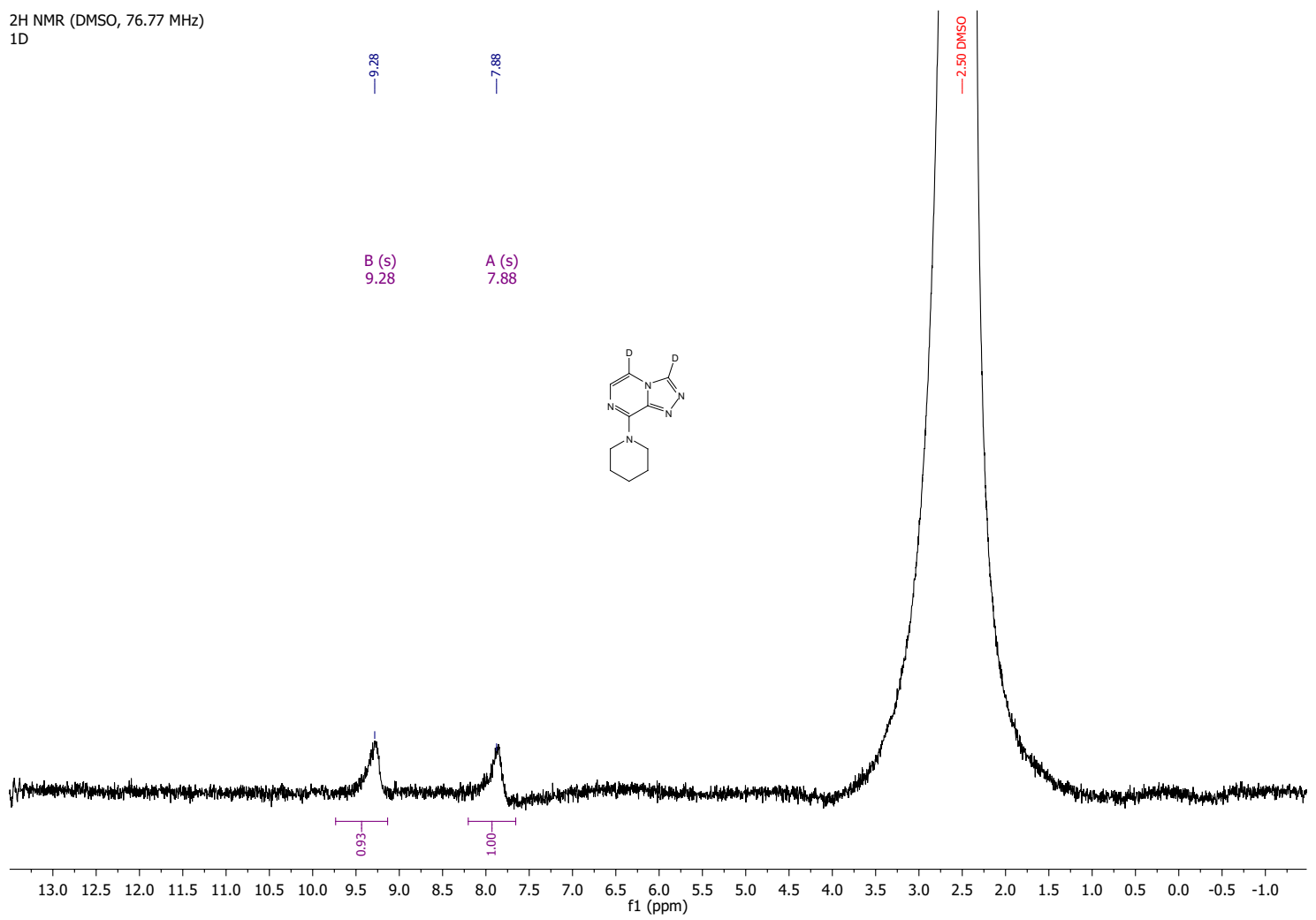


5-Chloro-[1,2,4] triazolo[4,3-a]pyrazine-3- $d 59$

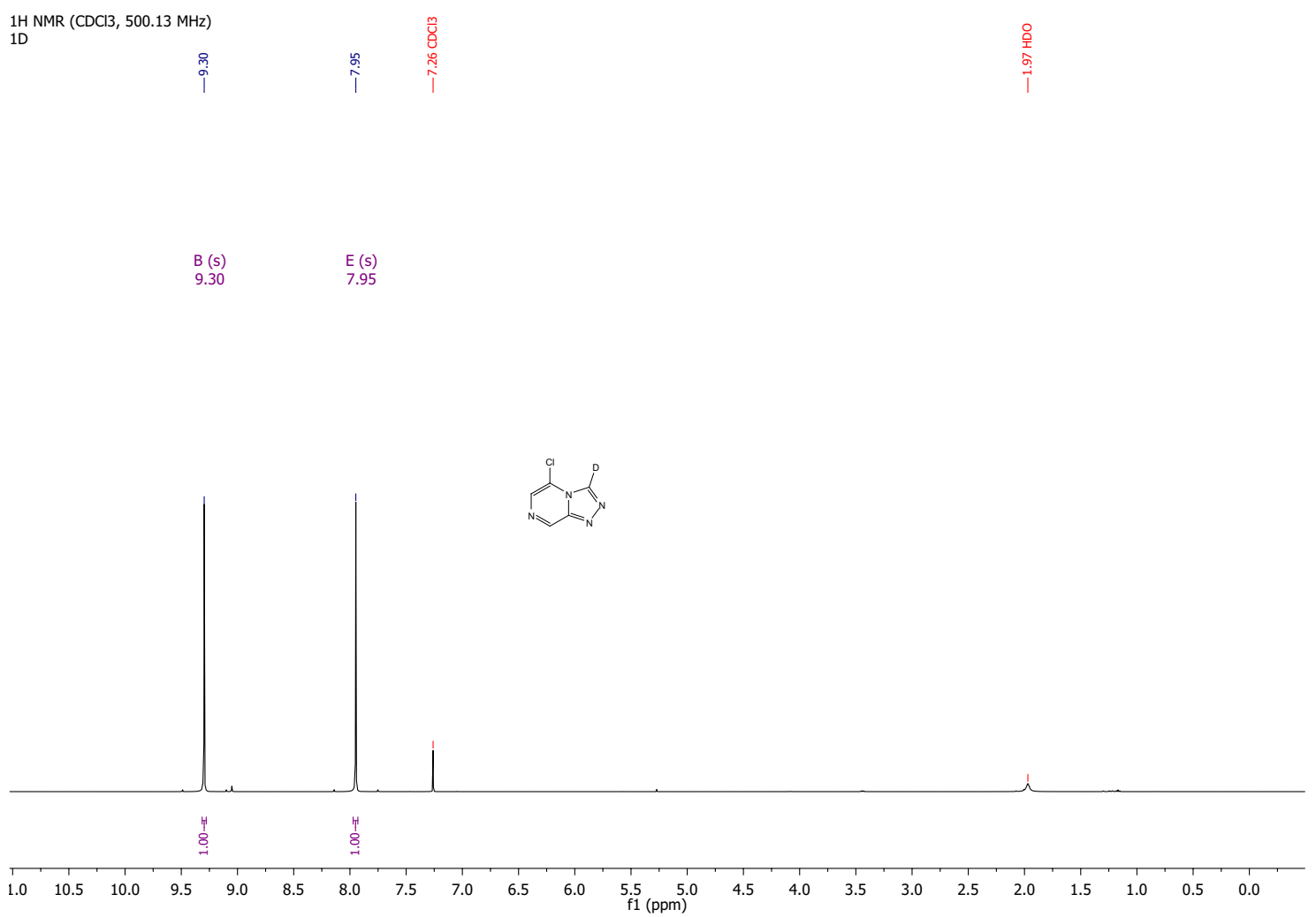

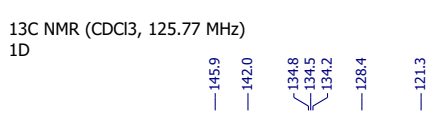
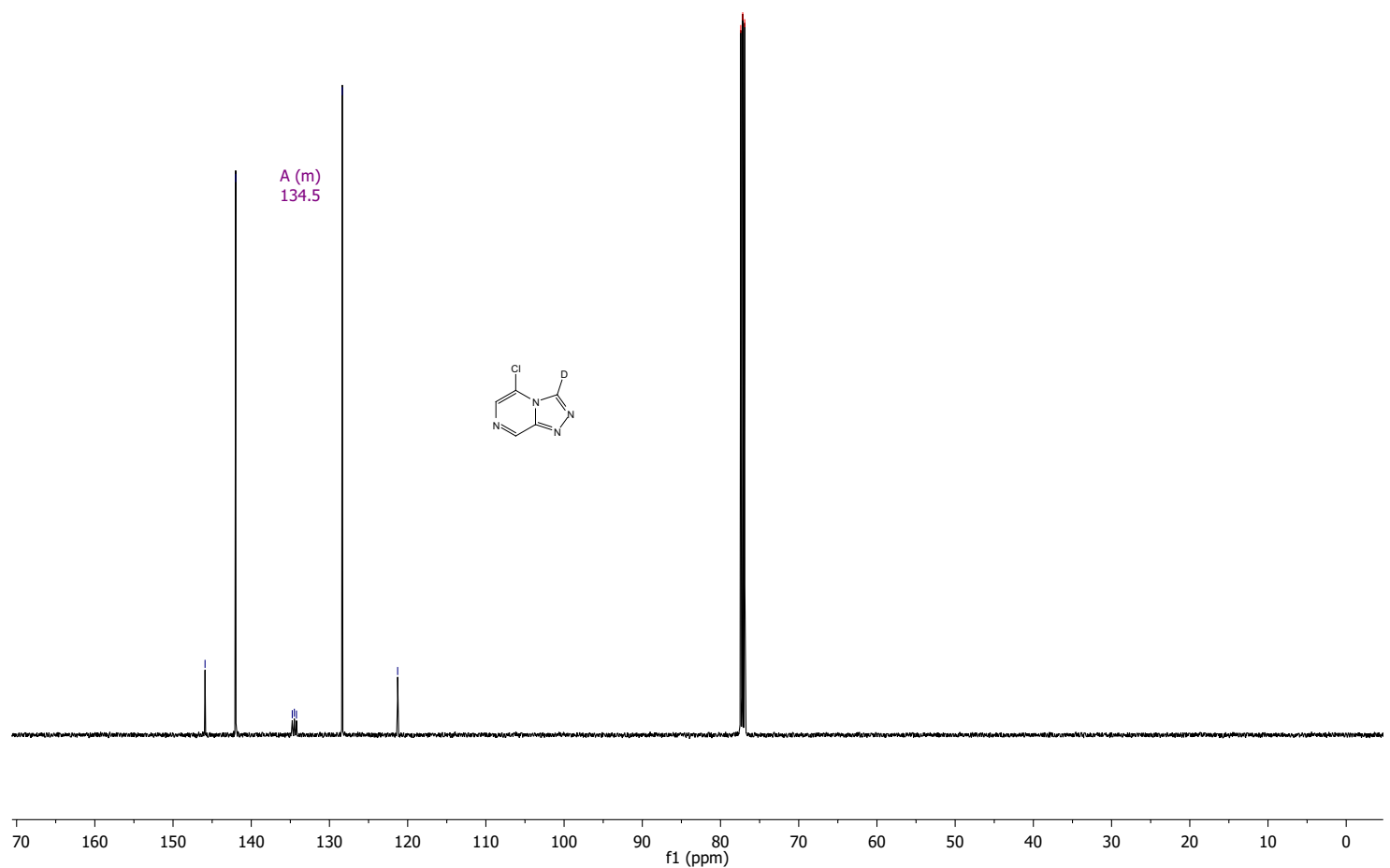


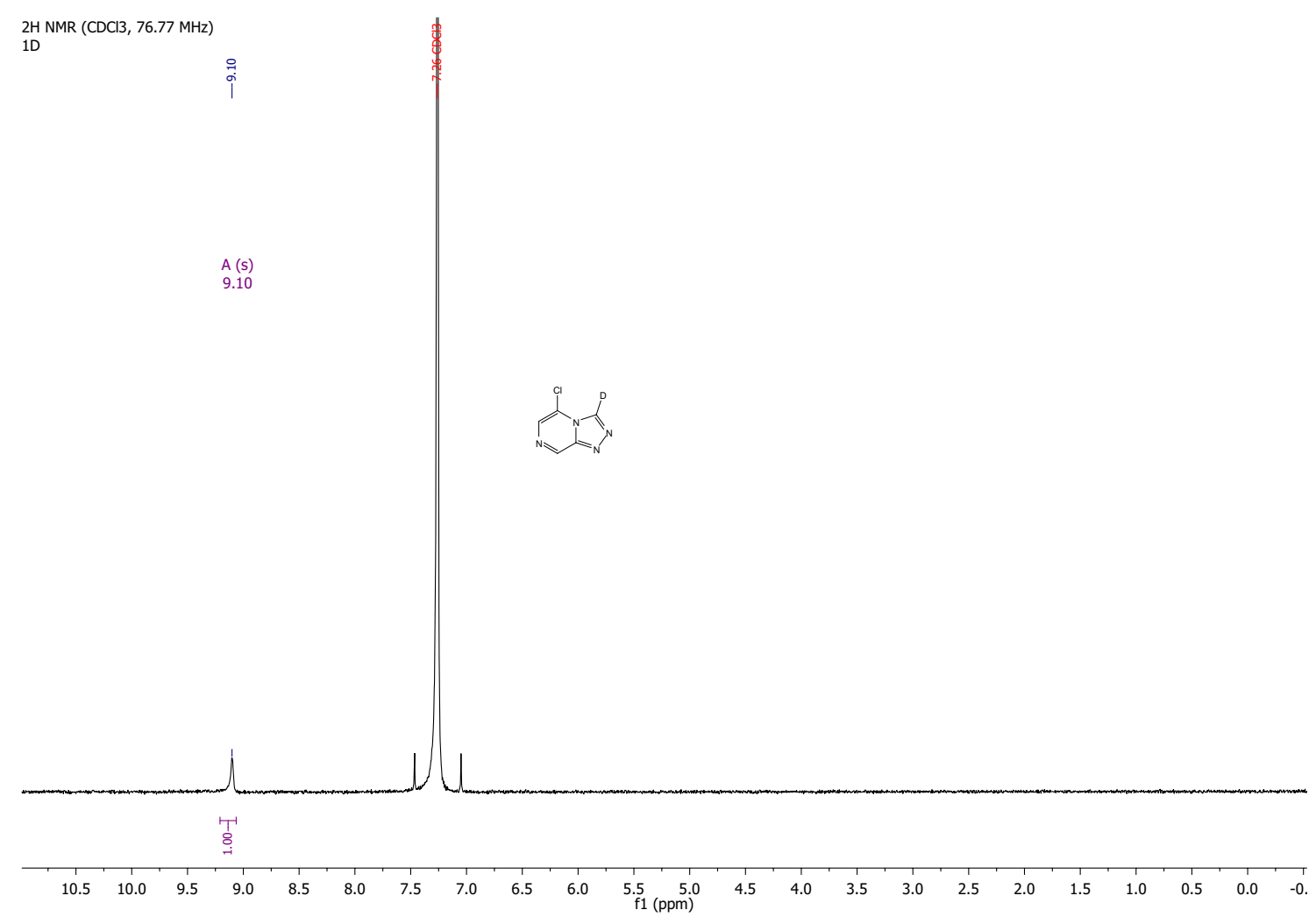


8-(Piperidin-1-yl)-[1,2,4]triazolo[4,3- $a]$ pyrazine-3- $d 60$

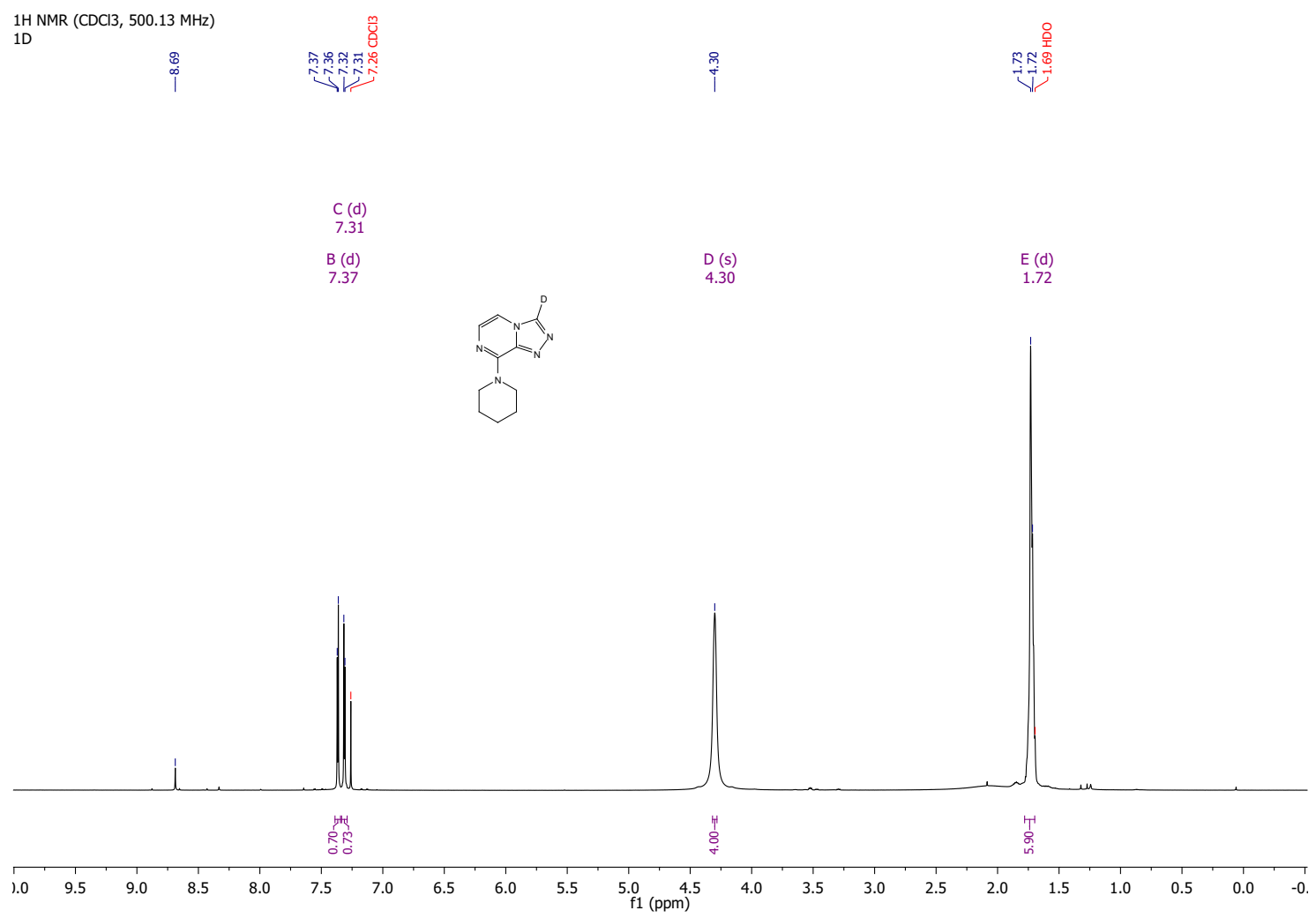

13C NMR (CDCl3, 125.77 MHz)

욤

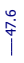

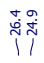
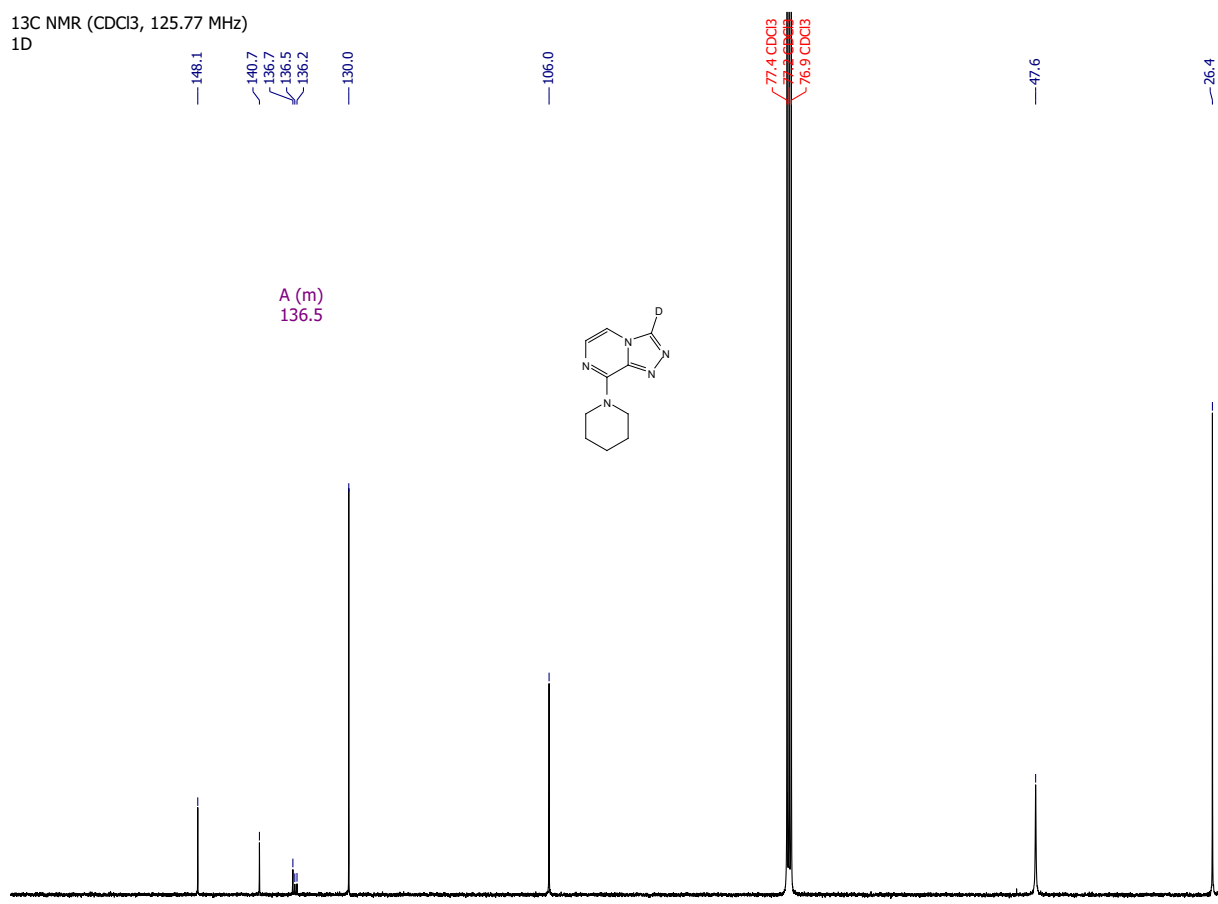

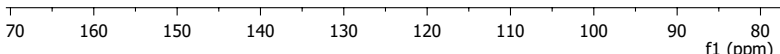




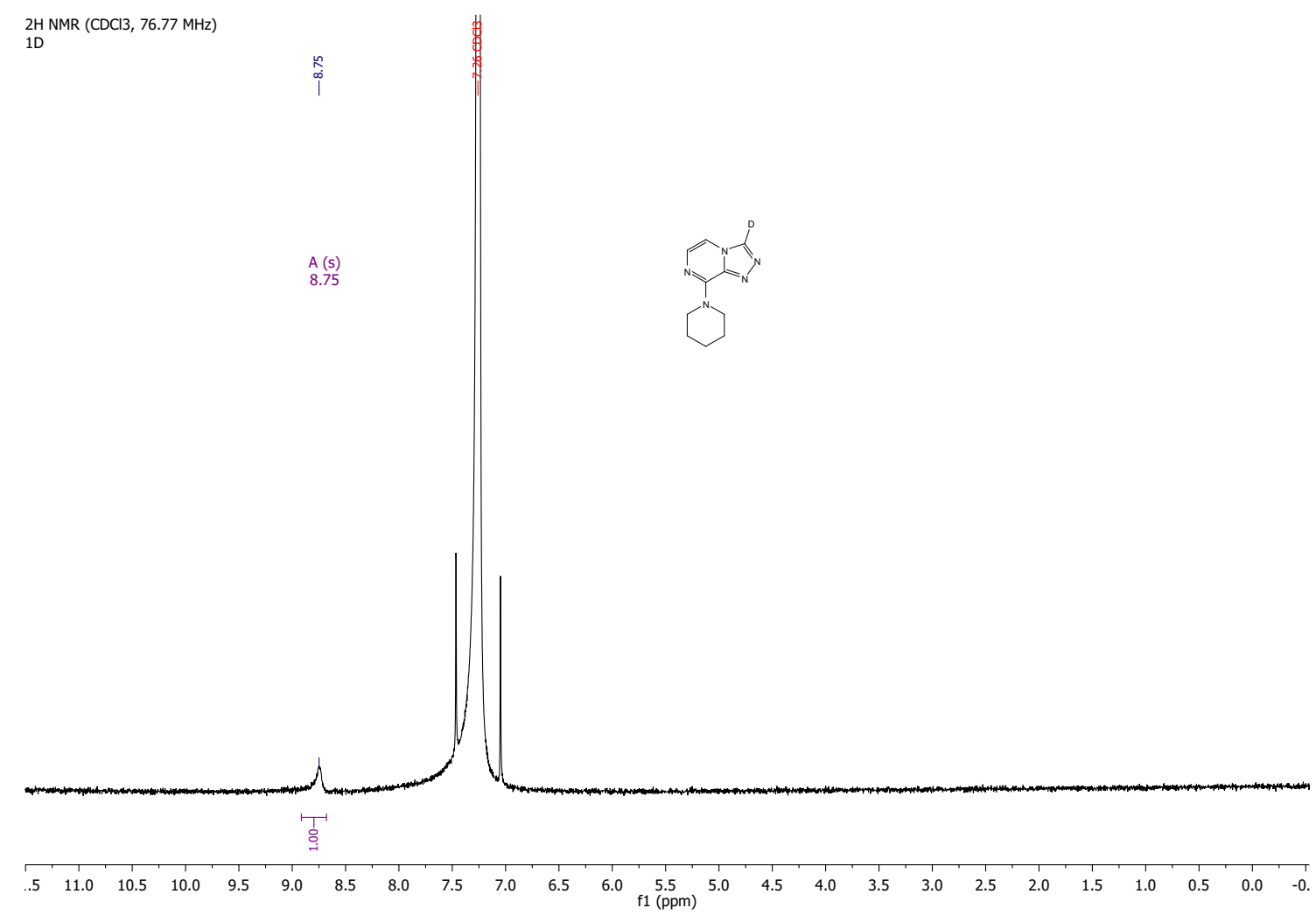




\section{S5 X-ray structures}

\section{S5.1 Crystal structure determination experimental parameters}

Single crystals of samples were obtained by slow evaporation from the correspondinging solvent, which is specified for each sample below. A suitable crystal was selected and attached with Paratone to a nylon loop. A SuperNova Dual equipped with an Atlas detector and employing mirror monochromated $\mathrm{Cu}(\mathrm{K} \alpha)$ radiation from a micro-source was used for the X-ray data collections. The crystal was kept at 100(2) K during data collection. Using Olex $2,{ }^{8}$ the structure was solved with the Olex2.solve ${ }^{9}$ structure solution program using Charge Flipping and refined with ShelXL ${ }^{10}$ and ShelXT ${ }^{11}$ refinement packages using Least Squares minimisation. Full information on crystal structure available free of charge from The Cambridge Crystallographic Data Centre via www.ccdc.cam.ac.uk/data_request/cif using correspondinging CCDC numbers provided for each compound. 


\section{S5.2 X-ray data and structure for $45 \mathrm{a}$}

Table S1: Crystal data and structure refinement for 45a

Crystallisation method

Empirical formula

Formula weight

Temperature/K

Crystal system

Space group

$\mathrm{a} / \AA$

$\mathrm{b} / \AA$

$\mathrm{c} / \AA$

$\alpha /{ }^{\circ}$

$\beta /{ }^{\circ}$

$\gamma /^{\circ}$

Volume $/ \AA^{3}$

$\mathrm{Z}$

$\rho_{\text {calc }} \mathrm{g} / \mathrm{cm}^{3}$

$\mu / \mathrm{mm}^{-1}$

$\mathrm{F}(000)$

Crystal size $/ \mathrm{mm}^{3}$

Radiation

$2 \Theta$ range for data collection/ ${ }^{\circ}$

Index ranges

Reflections collected

Independent reflections

Data/restraints/parameters

Goodness-of-fit on $\mathrm{F}^{2}$

Final $\mathrm{R}$ indexes $[\mathrm{I}>=2 \sigma(\mathrm{I})]$

Final $\mathrm{R}$ indexes [all data]

Largest diff. peak/hole/ e $\AA^{-3}$

CCDC Number
Slow evaporation from EtOAc solution $\mathrm{C}_{5} \mathrm{H}_{3} \mathrm{~N}_{4} \mathrm{Cl}$

154.56

$100(2)$

monoclinic

$\mathrm{P} 2{ }_{1} / \mathrm{m}$

$6.9527(2)$

$6.30259(16)$

$7.5416(2)$

90

$114.963(4)$

90

299.600(17)

2

1.713

4.929

156.0

$0.249 \times 0.072 \times 0.065$

$\operatorname{CuK} \alpha(\lambda=1.54184)$

12.948 to 151.992

$-8 \leq \mathrm{h} \leq 8,-7 \leq \mathrm{k} \leq 7,-8 \leq \mathrm{l} \leq 9$

5100

$686\left[\mathrm{R}_{\text {int }}=0.0178, \mathrm{R}_{\text {sigma }}=0.0086\right]$

$686 / 0 / 61$

1.123

$\mathrm{R}_{1}=0.0319, \mathrm{wR}_{2}=0.0832$

$\mathrm{R}_{1}=0.0319, \mathrm{wR}_{2}=0.0832$

$0.95 /-0.25$

1917945 
Figure S3: ORTEP depiction of $\mathbf{4 5 a}$

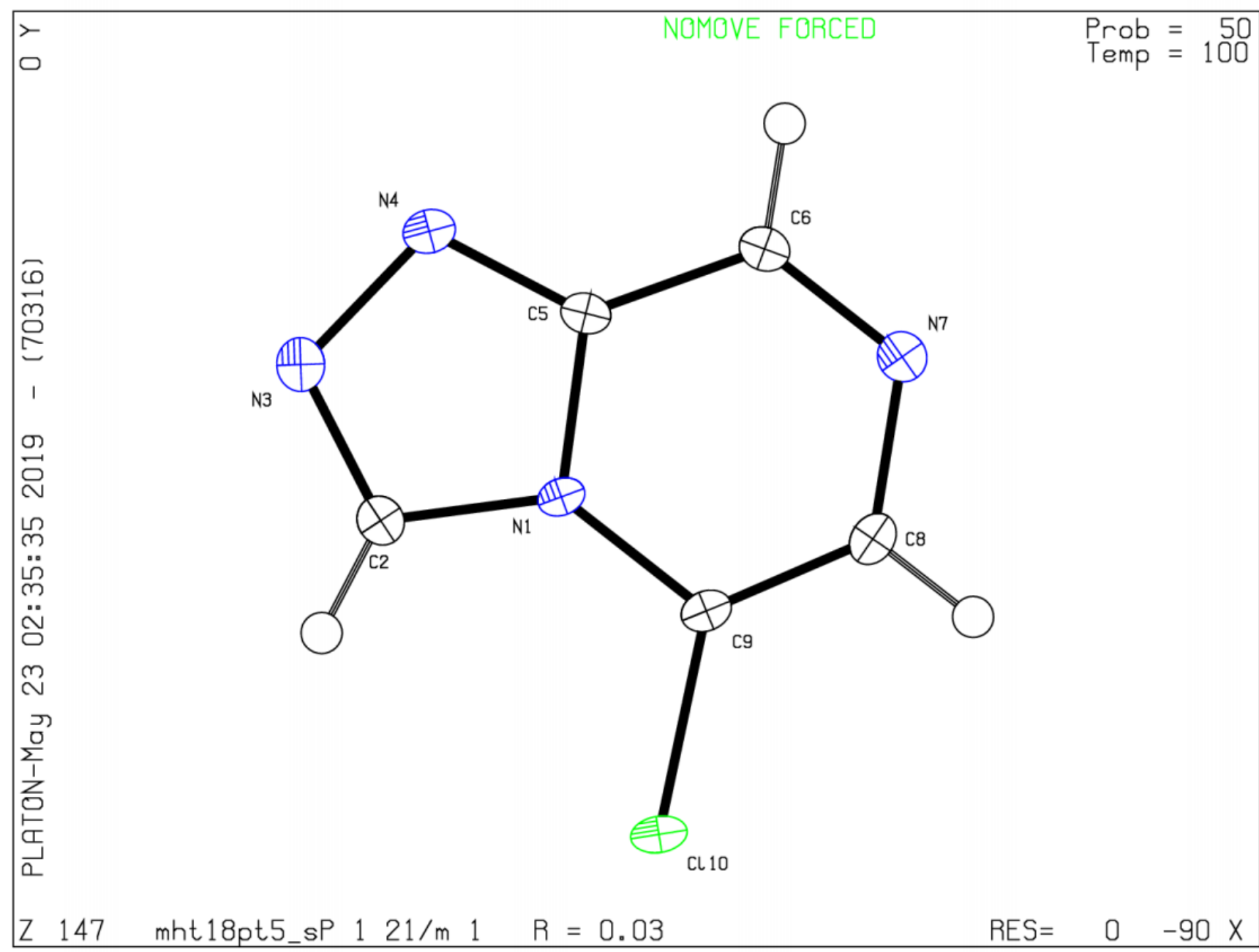




\section{S5.3 X-ray data and structure for 50}

Table S2: Crystal data and structure refinement for $\mathbf{5 0}$

Crystallisation method

Empirical formula

Formula weight

Temperature/K

Crystal system

Space group

$\mathrm{a} / \AA$

$\mathrm{b} / \AA$

$\mathrm{c} / \AA$

$\alpha /^{\circ}$

$\beta /{ }^{\circ}$

$\gamma /{ }^{\circ}$

Volume $/ \AA^{3}$

$\mathrm{Z}$

$\rho_{\text {calc }} / \mathrm{cm}^{3}$

$\mu / \mathrm{mm}^{-1}$

$\mathrm{F}(000)$

Crystal size $/ \mathrm{mm}^{3}$

Radiation

$2 \Theta$ range for data collection ${ }^{\circ}$

Index ranges

Reflections collected

Independent reflections

Data/restraints/parameters

Goodness-of-fit on $\mathrm{F}^{2}$

Final $\mathrm{R}$ indexes $[\mathrm{I}>=2 \sigma(\mathrm{I})]$

Final $\mathrm{R}$ indexes [all data]

Largest diff. peak/hole/ e $\AA^{-3}$

CCDC Number
Slow evaporation from EtOAc solution

$\mathrm{C}_{20} \mathrm{H}_{19} \mathrm{~N}_{5} \mathrm{O}$

345.40

$100(2)$

triclinic

P-1

$7.6546(6)$

$10.4869(7)$

$11.4484(8)$

$71.511(6)$

$82.118(6)$

$82.695(6)$

$859.90(11)$

2

1.334

0.693

364.0

$0.247 \times 0.061 \times 0.037$

$\mathrm{CuK} \alpha(\lambda=1.54184)$

8.186 to 145.928

$-9 \leq \mathrm{h} \leq 9,-12 \leq \mathrm{k} \leq 12,-13 \leq \mathrm{l} \leq 14$

12353

$3334\left[\mathrm{R}_{\text {int }}=0.0299, \mathrm{R}_{\text {sigma }}=0.0241\right]$

$3334 / 0 / 239$

1.031

$\mathrm{R}_{1}=0.0358, \mathrm{wR}_{2}=0.0938$

$\mathrm{R}_{1}=0.0417, \mathrm{wR}_{2}=0.0987$

$0.32 /-0.25$

$\mathbf{1 9 1 7 9 4 4}$ 
Figure S4: ORTEP depiction of $\mathbf{5 0}$

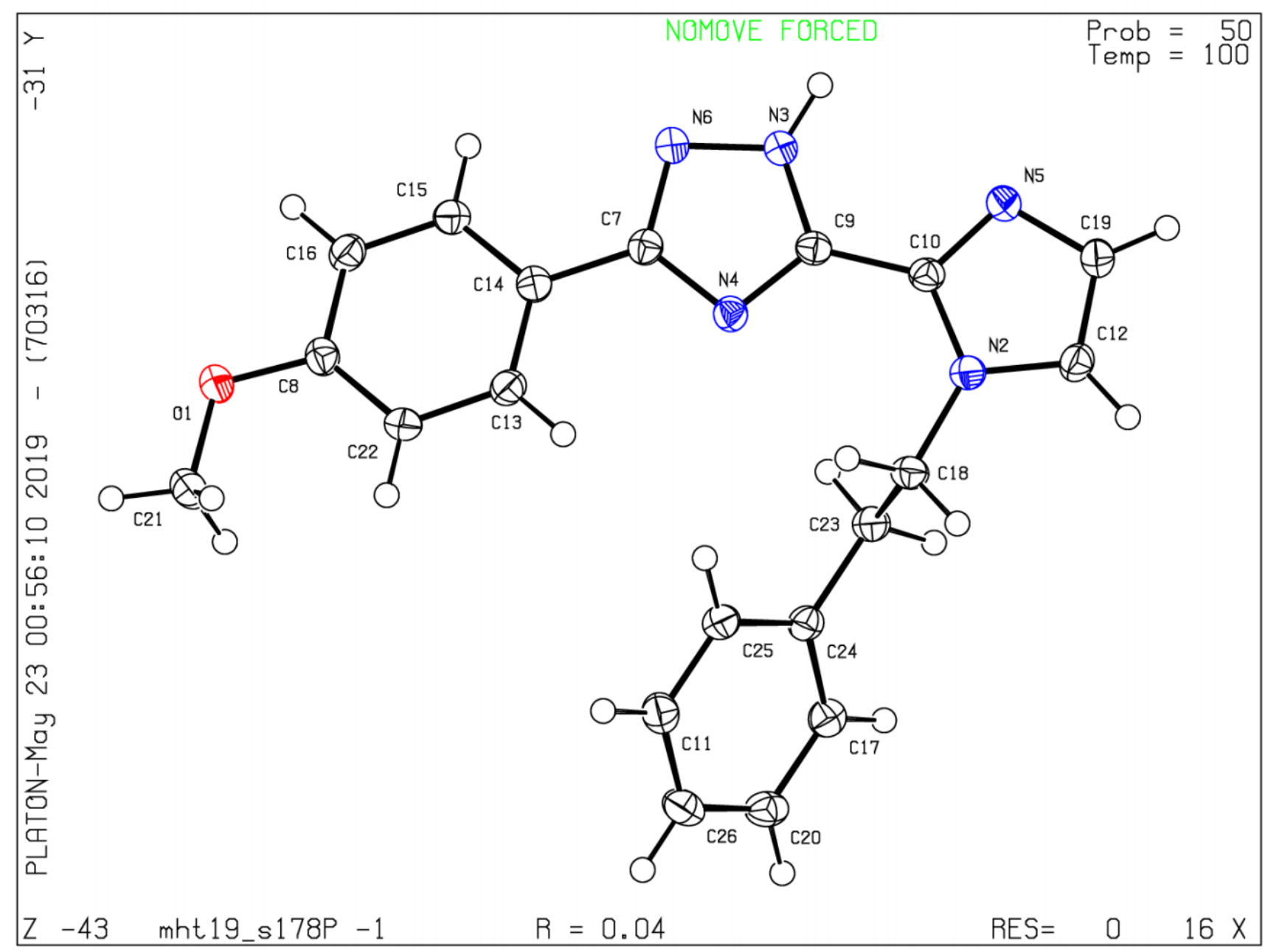




\section{S5.4 X-ray data and structure for 56}

Table S3: Crystal data and structure refinement for $\mathbf{5 6}$

Crystallisation method

Empirical formula

Formula weight

Temperature/K

Crystal system

Space group

$\mathrm{a} / \AA$

$\mathrm{b} / \AA$

$\mathrm{c} / \AA$

$\alpha /^{\circ}$

$\beta /{ }^{\circ}$

$\gamma /{ }^{\circ}$

Volume $/ \AA^{3}$

$\mathrm{Z}$

$\rho_{\text {calc }} / \mathrm{cm}^{3}$

$\mu / \mathrm{mm}^{-1}$

$\mathrm{F}(000)$

Crystal size $/ \mathrm{mm}^{3}$

Radiation

$2 \Theta$ range for data collection $/^{\circ}$

Index ranges

Reflections collected

Independent reflections

Data/restraints/parameters

Goodness-of-fit on $\mathrm{F}^{2}$

Final $\mathrm{R}$ indexes $[\mathrm{I}>=2 \sigma(\mathrm{I})]$

Final $\mathrm{R}$ indexes [all data]

Largest diff. peak/hole/ e $\AA^{-3}$

CCDC Number
Slow evaporation from EtOAc solution

$\mathrm{C}_{10} \mathrm{H}_{13} \mathrm{~N}_{5}$

203.25

$100(2)$

monoclinic

$\mathrm{P} 21 / \mathrm{c}$

$9.27494(16)$

$8.81723(13)$

$12.0155(2)$

90

$92.8415(17)$

90

$981.41(3)$

4

1.376

0.722

432.0

$0.246 \times 0.166 \times 0.1$

$\mathrm{CuK} \alpha(\lambda=1.54184)$

9.548 to 152.624

$-11 \leq \mathrm{h} \leq 11,-11 \leq \mathrm{k} \leq 11,-15 \leq \mathrm{l} \leq 14$

19400

$2040\left[\mathrm{R}_{\mathrm{int}}=0.0264, \mathrm{R}_{\mathrm{sigma}}=0.0118\right]$

$2040 / 0 / 136$

1.068

$\mathrm{R}_{1}=0.0323, \mathrm{wR}_{2}=0.0863$

$\mathrm{R}_{1}=0.0347, \mathrm{wR}_{2}=0.0889$

$0.30 /-0.24$

1917948 
Figure S5: ORTEP depiction of $\mathbf{5 6}$

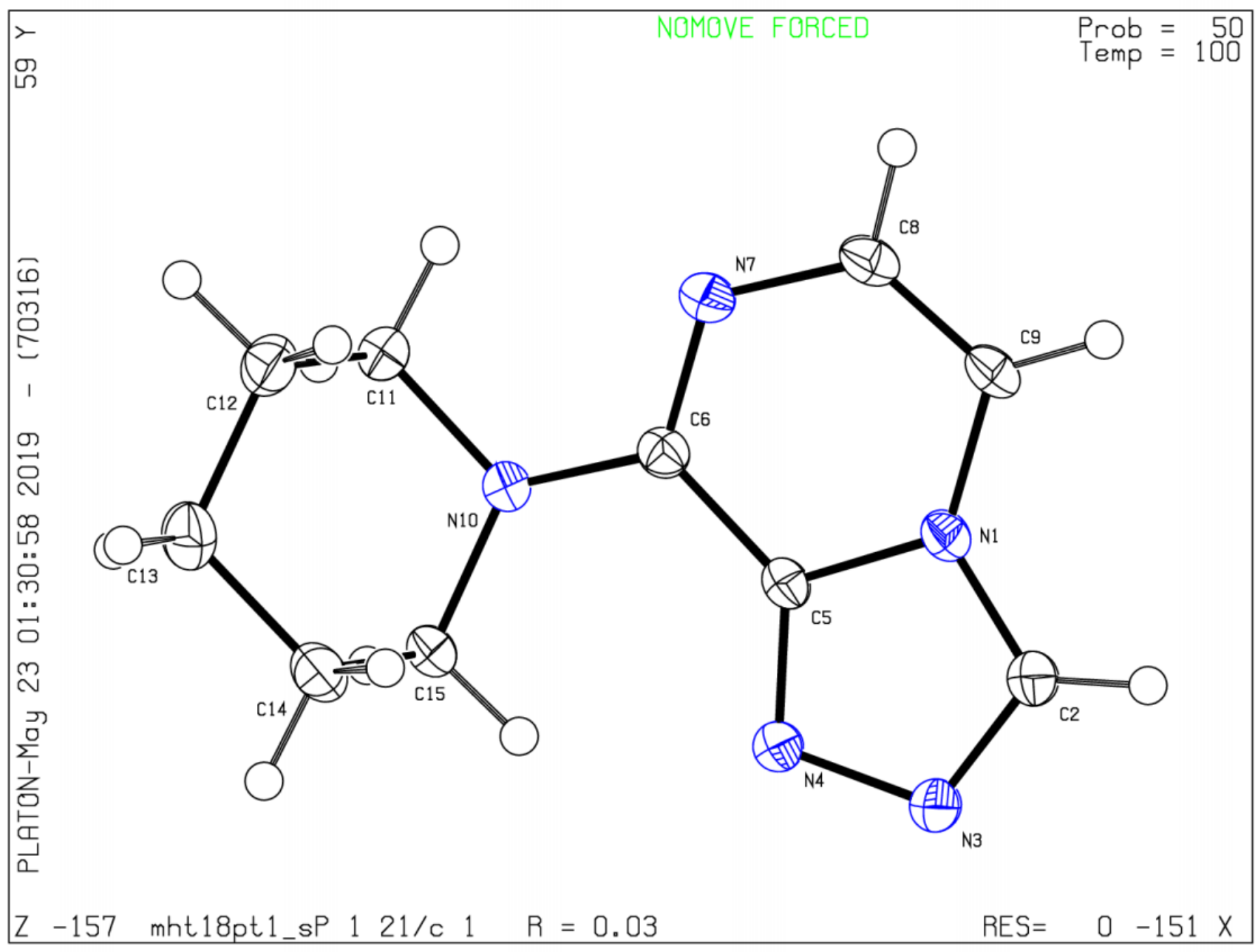




\section{References}

(1) Baragaña, B. et al. Lysyl-tRNA synthetase as a drug target in malaria and cryptosporidiosis. Proceedings of the National Academy of Sciences 2019, 116, 7015-7020.

(2) Miduturu, C. V.; Deng, X.; Kwiatkowski, N.; Yang, W.; Brault, L.; Filippakopoulos, P.; Chung, E.; Yang, Q.; Schwaller, J.; Knapp, S.; King, R. W.; Lee, J.-D.; Herrgard, S.; Zarrinkar, P.; Gray, N. S. High-Throughput Kinase Profiling: A More Efficient Approach toward the Discovery of New Kinase Inhibitors. Chemistry \& Biology 2011, 18, 868-879.

(3) Fabian, M. A. et al. A small molecule-kinase interaction map for clinical kinase inhibitors. Nature Biotechnology 2005, 23, 329-336.

(4) Wodicka, L. M.; Ciceri, P.; Davis, M. I.; Hunt, J. P.; Floyd, M.; Salerno, S.; Hua, X. H.; Ford, J. M.; Armstrong, R. C.; Zarrinkar, P. P.; Treiber, D. K. Activation State-Dependent Binding of Small Molecule Kinase Inhibitors: Structural Insights from Biochemistry. Chemistry \& Biology 2010, 17, 1241-1249.

(5) Baldwin, J. E. Rules for ring closure. Journal of the Chemical Society, Chemical Communications 1976, 734 .

(6) Baldwin, J. E.; Cutting, J.; Dupont, W.; Kruse, L.; Silberman, L.; Thomas, R. C. 5-Endotrigonal reactions: a disfavoured ring closure. Journal of the Chemical Society, Chemical Communications 1976, 736.

(7) Gilmore, K.; Manoharan, M.; Wu, J. I.-C.; v. R. Schleyer, P.; Alabugin, I. V. Aromatic Transition States in Nonpericyclic Reactions: Anionic 5-Endo Cyclizations Are Aborted Sigmatropic Shifts. Journal of the American Chemical Society 2012, 134, 10584-10594.

(8) Dolomanov, O. V.; Bourhis, L. J.; Gildea, R. J.; Howard, J. A. K.; Puschmann, H. OLEX2: a complete structure solution, refinement and analysis program. Journal of Applied Crystallography 2009, 42, 339-341.

(9) Bourhis, L. J.; Dolomanov, O. V.; Gildea, R. J.; Howard, J. A. K.; Puschmann, H. The anatomy of a comprehensive constrained, restrained refinement program for the modern computing environment - Olex2dissected. Acta Crystallographica Section A Foundations and Advances 2015, 71, 59-75.

(10) Sheldrick, G. M. Crystal structure refinement with SHELXL. Acta Crystallographica Section C Structural Chemistry 2015, 71, 3-8.

(11) Sheldrick, G. M. SHELXT - Integrated space-group and crystal-structure determination. Acta Crystallographica Section A Foundations and Advances 2015, 71, 3-8. 


\begin{tabular}{|c|c|c|}
\hline Paper code & ELN code & SMILES \\
\hline s1 & MK001 & $\mathrm{ClC} 1=\mathrm{CN}=\mathrm{CC}(\mathrm{NN})=\mathrm{N} 1$ \\
\hline S2 & MK102 & $\mathrm{NNC} 1=\mathrm{NC}(\mathrm{Br})=\mathrm{CN}=\mathrm{C} 1$ \\
\hline S3 & MK124 & $\mathrm{IC} 1=\mathrm{CN}=\mathrm{CC}(\mathrm{NN})=\mathrm{N} 1$ \\
\hline S4 & MK092 & $\mathrm{NNC} 1=\mathrm{NC}=\mathrm{CN}=\mathrm{C} 1 \mathrm{Cl}$ \\
\hline S5 & MK097 & $\mathrm{NNC} 1=\mathrm{NC}=\mathrm{C}(\mathrm{Cl}) \mathrm{N}=\mathrm{C} 1$ \\
\hline 16 & MK004 & $\mathrm{ClC} 1=\mathrm{CN}=\mathrm{CC} 2=\mathrm{NN}=\mathrm{C}(\mathrm{C} 3=\mathrm{CC}=\mathrm{C}(\mathrm{OC}(\mathrm{F}) \mathrm{F}) \mathrm{C}=\mathrm{C} 3) \mathrm{N} 21$ \\
\hline 17 & MK087a & $\begin{array}{l}\mathrm{FC}(\mathrm{F}) \mathrm{OC}(\mathrm{C}=\mathrm{C} 1)=\mathrm{CC}=\mathrm{C} 1 \mathrm{C} 2=\mathrm{NN}=\mathrm{C} 3 \mathrm{C}=\mathrm{NC}=\mathrm{C}(\mathrm{SCCC} 4= \\
\mathrm{CC}=\mathrm{CC}=\mathrm{C} 4) \mathrm{N} 32\end{array}$ \\
\hline 18 & MK087b & $\begin{array}{l}\mathrm{FC}(\mathrm{F}) \mathrm{OC}(\mathrm{C}=\mathrm{C} 1)=\mathrm{CC}=\mathrm{C} 1 \mathrm{C} 2=\mathrm{NN}=\mathrm{C} 3 \mathrm{C}(\mathrm{SCCC} 4=\mathrm{CC}=\mathrm{CC} \\
=\mathrm{C} 4)=\mathrm{NC}=\mathrm{CN} 32\end{array}$ \\
\hline 25 & MK093 & $\mathrm{ClC} 1=\mathrm{NC}=\mathrm{CN} 2 \mathrm{C} 1=\mathrm{NN}=\mathrm{C} 2$ \\
\hline 26 & MK134 & $\mathrm{ClC} 1=\mathrm{NC}=\mathrm{CN} 2 \mathrm{C} 1=\mathrm{NN}=\mathrm{C} 2 \mathrm{C} 3=\mathrm{CC}=\mathrm{CC}=\mathrm{C} 3$ \\
\hline 27 & MK133 & $\begin{array}{l}{[\mathrm{O}-} \\
][\mathrm{N}+](\mathrm{C} 1=\mathrm{CC}=\mathrm{C}(\mathrm{C} 2=\mathrm{NN}=\mathrm{C} 3 \mathrm{C}(\mathrm{Cl})=\mathrm{NC}=\mathrm{CN} 32) \mathrm{C}=\mathrm{C} 1)=\mathrm{O}\end{array}$ \\
\hline 28 & MK096 & $\mathrm{C} 1(\mathrm{OCCC} 2=\mathrm{CC}=\mathrm{CC}=\mathrm{C} 2)=\mathrm{NC}=\mathrm{CN} 3 \mathrm{C} 1=\mathrm{NN}=\mathrm{C} 3$ \\
\hline 29 & MK094 & $\mathrm{C} 1(\mathrm{SCCC} 2=\mathrm{CC}=\mathrm{CC}=\mathrm{C} 2)=\mathrm{NC}=\mathrm{CN} 3 \mathrm{C} 1=\mathrm{NN}=\mathrm{C} 3$ \\
\hline 30 & MK085 & $\mathrm{C} 1(\mathrm{NCCC} 2=\mathrm{CC}=\mathrm{CC}=\mathrm{C} 2)=\mathrm{NC}=\mathrm{CN} 3 \mathrm{C} 1=\mathrm{NN}=\mathrm{C} 3$ \\
\hline 31 & MK113b & $\begin{array}{l}\mathrm{C} 1(\mathrm{OCCC} 2=\mathrm{CC}=\mathrm{CC}=\mathrm{C} 2)=\mathrm{NC}=\mathrm{CN} 3 \mathrm{C} 1=\mathrm{NN}=\mathrm{C} 3 \mathrm{C} 4=\mathrm{CC} \\
=\mathrm{CC}=\mathrm{C} 4\end{array}$ \\
\hline 32 & MK110b & $\begin{array}{l}\mathrm{C} 1(\mathrm{SCCC} 2=\mathrm{CC}=\mathrm{CC}=\mathrm{C} 2)=\mathrm{NC}=\mathrm{CN} 3 \mathrm{C} 1=\mathrm{NN}=\mathrm{C} 3 \mathrm{C} 4=\mathrm{CC}= \\
\mathrm{CC}=\mathrm{C} 4\end{array}$ \\
\hline 33 & MK116 & $\begin{array}{l}\mathrm{C} 1(\mathrm{NCCC} 2=\mathrm{CC}=\mathrm{CC}=\mathrm{C} 2)=\mathrm{NC}=\mathrm{CN} 3 \mathrm{C} 1=\mathrm{NN}=\mathrm{C} 3 \mathrm{C} 4=\mathrm{CC} \\
=\mathrm{CC}=\mathrm{C} 4\end{array}$ \\
\hline 34 & MK115b & $\begin{array}{l}\mathrm{O}=[\mathrm{N}+](\mathrm{C}(\mathrm{C}=\mathrm{C} 1)=\mathrm{CC}=\mathrm{C} 1 \mathrm{C} 2=\mathrm{NN}=\mathrm{C} 3 \mathrm{C}(\mathrm{OCCC} 4=\mathrm{CC}=\mathrm{C} \\
\mathrm{C}=\mathrm{C} 4)=\mathrm{NC}=\mathrm{CN} 32)[\mathrm{O}-]\end{array}$ \\
\hline 35 & MK112b & $\begin{array}{l}\mathrm{O}=[\mathrm{N}+](\mathrm{C}(\mathrm{C}=\mathrm{C} 1)=\mathrm{CC}=\mathrm{C} 1 \mathrm{C} 2=\mathrm{NN}=\mathrm{C} 3 \mathrm{C}(\mathrm{SCCC} 4=\mathrm{CC}=\mathrm{C} \\
\mathrm{C}=\mathrm{C} 4)=\mathrm{NC}=\mathrm{CN} 32)[\mathrm{O}-]\end{array}$ \\
\hline 36 & MK118 & $\begin{array}{l}\mathrm{O}=[\mathrm{N}+](\mathrm{C}(\mathrm{C}=\mathrm{C} 1)=\mathrm{CC}=\mathrm{C} 1 \mathrm{C} 2=\mathrm{NN}=\mathrm{C} 3 \mathrm{C}(\mathrm{NCCC} 4=\mathrm{CC}=\mathrm{C} \\
\mathrm{C}=\mathrm{C} 4)=\mathrm{NC}=\mathrm{CN} 32)[\mathrm{O}-]\end{array}$ \\
\hline 37 & MK098 & $\mathrm{ClC}(\mathrm{N}=\mathrm{C} 1)=\mathrm{CN} 2 \mathrm{C} 1=\mathrm{NN}=\mathrm{C} 2$ \\
\hline 38 & MK145 & $\mathrm{ClC}(\mathrm{N}=\mathrm{C} 1)=\mathrm{CN} 2 \mathrm{C} 1=\mathrm{NN}=\mathrm{C} 2 \mathrm{C} 3=\mathrm{CC}=\mathrm{C}(\mathrm{OC}(\mathrm{F}) \mathrm{F}) \mathrm{C}=\mathrm{C} 3$ \\
\hline 41 & MK123 & $\mathrm{IC} 1=\mathrm{NC}(\mathrm{I})=\mathrm{CN}=\mathrm{C} 1$ \\
\hline 42 & MK141a & $\mathrm{ClC} 1=\mathrm{NC}(\mathrm{OCCC} 2=\mathrm{CC}=\mathrm{CC}=\mathrm{C} 2)=\mathrm{CN}=\mathrm{C} 1$ \\
\hline 43 & MK143 & $\mathrm{BrC} 1=\mathrm{NC}(\mathrm{OCCC} 2=\mathrm{CC}=\mathrm{CC}=\mathrm{C} 2)=\mathrm{CN}=\mathrm{C} 1$ \\
\hline 44 & MK144 & IC $1=\mathrm{NC}(\mathrm{OCCC} 2=\mathrm{CC}=\mathrm{CC}=\mathrm{C} 2)=\mathrm{CN}=\mathrm{C} 1$ \\
\hline
\end{tabular}




\begin{tabular}{|c|c|c|}
\hline $45 a$ & MK002 & $\mathrm{ClC} 1=\mathrm{CN}=\mathrm{CC} 2=\mathrm{NN}=\mathrm{CN} 21$ \\
\hline 45b & MK108 & $\mathrm{ClC} 1=\mathrm{CN}=\mathrm{CC}(\mathrm{N} 12)=\mathrm{NN}=\mathrm{C} 2 \mathrm{C} 3=\mathrm{CC}=\mathrm{C}(\mathrm{OC}) \mathrm{C}=\mathrm{C} 3$ \\
\hline $45 c$ & MK109 & $\begin{array}{l}\mathrm{ClC} 1=\mathrm{CN}=\mathrm{CC}(\mathrm{N} 12)=\mathrm{NN}=\mathrm{C} 2 \mathrm{C} 3=\mathrm{CC}=\mathrm{C}([\mathrm{N}+]([\mathrm{O}- \\
])=\mathrm{O}) \mathrm{C}=\mathrm{C} 3\end{array}$ \\
\hline 45d & SSP 2019D1 & $\mathrm{ClC} 1=\mathrm{CN}=\mathrm{CC}(\mathrm{N} 12)=\mathrm{NN}=\mathrm{C} 2 \mathrm{C} 3=\mathrm{C}(\mathrm{OC}) \mathrm{C}=\mathrm{CC}=\mathrm{C} 3$ \\
\hline $45 e$ & MK168 & $\begin{array}{l}\mathrm{ClC} 1=\mathrm{CN}=\mathrm{CC}(\mathrm{N} 12)=\mathrm{NN}=\mathrm{C} 2 \mathrm{C} 3=\mathrm{C}([\mathrm{N}+]([\mathrm{O}- \\
])=\mathrm{O}) \mathrm{C}=\mathrm{CC}=\mathrm{C} 3\end{array}$ \\
\hline $45 f$ & MK175 & $\begin{array}{l}\mathrm{ClC} 1=\mathrm{CN}=\mathrm{CC} 2=\mathrm{NN}=\mathrm{C}(\mathrm{C} 3=\mathrm{CC}(\mathrm{C}(\mathrm{C})(\mathrm{C}) \mathrm{C})=\mathrm{CC}(\mathrm{C}(\mathrm{C})(\mathrm{C}) \\
\mathrm{C})=\mathrm{C} 3) \mathrm{N} 21\end{array}$ \\
\hline $45 \mathrm{~g}$ & MK170 & $\begin{array}{l}\mathrm{ClC} 1=\mathrm{CN}=\mathrm{CC}(\mathrm{N} 12)=\mathrm{NN}=\mathrm{C} 2 \mathrm{C} 3=\mathrm{C}(\mathrm{C}=\mathrm{CC}=\mathrm{C} 4) \mathrm{C} 4=\mathrm{CC} 5 \\
=\mathrm{C} 3 \mathrm{C}=\mathrm{CC}=\mathrm{C} 5\end{array}$ \\
\hline $45 \mathrm{~h}$ & MK103 & $\mathrm{BrC} 1=\mathrm{CN}=\mathrm{CC} 2=\mathrm{NN}=\mathrm{CN} 21$ \\
\hline $45 i$ & MK119 & $\mathrm{BrC} 1=\mathrm{CN}=\mathrm{CC}(\mathrm{N} 12)=\mathrm{NN}=\mathrm{C} 2 \mathrm{C} 3=\mathrm{CC}=\mathrm{C}(\mathrm{OC}) \mathrm{C}=\mathrm{C} 3$ \\
\hline $45 j$ & MK183 & $\begin{array}{l}\mathrm{BrC} 1=\mathrm{CN}=\mathrm{CC}(\mathrm{N} 12)=\mathrm{NN}=\mathrm{C} 2 \mathrm{C} 3=\mathrm{CC}=\mathrm{C}([\mathrm{N}+]([\mathrm{O}- \\
])=\mathrm{O}) \mathrm{C}=\mathrm{C} 3\end{array}$ \\
\hline 45k & MK181 & $\mathrm{BrC} 1=\mathrm{CN}=\mathrm{CC}(\mathrm{N} 12)=\mathrm{NN}=\mathrm{C} 2 \mathrm{C} 3=\mathrm{C}(\mathrm{OC}) \mathrm{C}=\mathrm{CC}=\mathrm{C} 3$ \\
\hline 45I & MK185 & $\begin{array}{l}\mathrm{BrC} 1=\mathrm{CN}=\mathrm{CC}(\mathrm{N} 12)=\mathrm{NN}=\mathrm{C} 2 \mathrm{C} 3=\mathrm{C}([\mathrm{N}+]([\mathrm{O}- \\
])=\mathrm{O}) \mathrm{C}=\mathrm{CC}=\mathrm{C} 3\end{array}$ \\
\hline $45 m$ & MK129 & $\mathrm{IC} 1=\mathrm{CN}=\mathrm{CC} 2=\mathrm{NN}=\mathrm{CN} 21$ \\
\hline $45 n$ & MK125 & $\mathrm{IC} 1=\mathrm{CN}=\mathrm{CC}(\mathrm{N} 12)=\mathrm{NN}=\mathrm{C} 2 \mathrm{C} 3=\mathrm{CC}=\mathrm{C}(\mathrm{OC}) \mathrm{C}=\mathrm{C} 3$ \\
\hline $46 a$ & MK006 & $\mathrm{C} 12=\mathrm{NN}=\mathrm{CN} 1 \mathrm{C}(\mathrm{OCCC} 3=\mathrm{CC}=\mathrm{CC}=\mathrm{C} 3)=\mathrm{CN}=\mathrm{C} 2$ \\
\hline $46 b$ & MK086 & $\mathrm{C} 12=\mathrm{NN}=\mathrm{CN} 1 \mathrm{C}(\mathrm{SCCC} 3=\mathrm{CC}=\mathrm{CC}=\mathrm{C} 3)=\mathrm{CN}=\mathrm{C} 2$ \\
\hline $46 c$ & MK114a & $\begin{array}{l}\mathrm{COC}(\mathrm{C}=\mathrm{C} 1)=\mathrm{CC}=\mathrm{C} 1 \mathrm{C} 2=\mathrm{NN}=\mathrm{C} 3 \mathrm{C}=\mathrm{NC}=\mathrm{C}(\mathrm{OCCC} 4=\mathrm{CC} \\
=\mathrm{CC}=\mathrm{C} 4) \mathrm{N} 32\end{array}$ \\
\hline 46d & MK111a & $\begin{array}{l}\mathrm{COC}(\mathrm{C}=\mathrm{C} 1)=\mathrm{CC}=\mathrm{C} 1 \mathrm{C} 2=\mathrm{NN}=\mathrm{C} 3 \mathrm{C}=\mathrm{NC}=\mathrm{C}(\mathrm{SCCC} 4=\mathrm{CC}= \\
\mathrm{CC}=\mathrm{C} 4) \mathrm{N} 32\end{array}$ \\
\hline $46 e$ & MK115a & $\begin{array}{l}\mathrm{O}=[\mathrm{N}+](\mathrm{C}(\mathrm{C}=\mathrm{C} 1)=\mathrm{CC}=\mathrm{C} 1 \mathrm{C} 2=\mathrm{NN}=\mathrm{C} 3 \mathrm{C}=\mathrm{NC}=\mathrm{C}(\mathrm{OCCC} 4 \\
=\mathrm{CC}=\mathrm{CC}=\mathrm{C} 4) \mathrm{N} 32)[\mathrm{O}-]\end{array}$ \\
\hline $46 f$ & MK182a & $\begin{array}{l}\mathrm{COC} 1=\mathrm{C}(\mathrm{C} 2=\mathrm{NN}=\mathrm{C} 3 \mathrm{C}=\mathrm{NC}=\mathrm{C}(\mathrm{OCCC} 4=\mathrm{CC}=\mathrm{CC}=\mathrm{C} 4) \mathrm{N} \\
32) \mathrm{C}=\mathrm{CC}=\mathrm{C} 1\end{array}$ \\
\hline $46 \mathrm{~g}$ & MK169 & $\begin{array}{l}\mathrm{O}=[\mathrm{N}+](\mathrm{C}(\mathrm{C}=\mathrm{CC}=\mathrm{C} 1)=\mathrm{C} 1 \mathrm{C} 2=\mathrm{NN}=\mathrm{C} 3 \mathrm{C}=\mathrm{NC}=\mathrm{C}(\mathrm{OCCC} 4 \\
=\mathrm{CC}=\mathrm{CC}=\mathrm{C} 4) \mathrm{N} 32)[\mathrm{O}-]\end{array}$ \\
\hline $46 \mathrm{~h}$ & MK176a & $\begin{array}{l}C C(C)(C) C 1=C C(C 2=N N=C 3 C=N C=C(O C C C 4=C C=C \\
C=C 4) N 32)=C C(C(C)(C) C)=C 1\end{array}$ \\
\hline $46 i$ & MK171a & $\begin{array}{l}\mathrm{C} 12=\mathrm{NN}=\mathrm{C}(\mathrm{C} 3=\mathrm{C} 4 \mathrm{C}=\mathrm{CC}=\mathrm{CC} 4=\mathrm{CC} 5=\mathrm{C} 3 \mathrm{C}=\mathrm{CC}=\mathrm{C} 5) \mathrm{N} 1 \\
\mathrm{C}(\mathrm{OCCC} 6=\mathrm{CC}=\mathrm{CC}=\mathrm{C} 6)=\mathrm{CN}=\mathrm{C} 2\end{array}$ \\
\hline $47 a$ & MK114b & $\begin{array}{l}\mathrm{COC}(\mathrm{C}=\mathrm{C} 1)=\mathrm{CC}=\mathrm{C} 1 \mathrm{C} 2=\mathrm{NN}=\mathrm{C} 3 \mathrm{C}(\mathrm{OCCC} 4=\mathrm{CC}=\mathrm{CC}=\mathrm{C} \\
4)=\mathrm{NC}=\mathrm{CN} 32\end{array}$ \\
\hline
\end{tabular}




\begin{tabular}{|c|c|c|}
\hline $47 \mathrm{~b}$ & MK111b & $\begin{array}{l}\mathrm{COC}(\mathrm{C}=\mathrm{C} 1)=\mathrm{CC}=\mathrm{C} 1 \mathrm{C} 2=\mathrm{NN}=\mathrm{C} 3 \mathrm{C}(\mathrm{SCCC} 4=\mathrm{CC}=\mathrm{CC}=\mathrm{C} 4 \\
)=\mathrm{NC}=\mathrm{CN} 32\end{array}$ \\
\hline 47c & MK117 & $\begin{array}{l}\mathrm{COC}(\mathrm{C}=\mathrm{C} 1)=\mathrm{CC}=\mathrm{C} 1 \mathrm{C} 2=\mathrm{NN}=\mathrm{C} 3 \mathrm{C}(\mathrm{NCCC} 4=\mathrm{CC}=\mathrm{CC}=\mathrm{C} \\
4)=\mathrm{NC}=\mathrm{CN} 32\end{array}$ \\
\hline 47d & MK176b & $\begin{array}{l}\mathrm{CC}(\mathrm{C})(\mathrm{C}) \mathrm{C} 1=\mathrm{CC}(\mathrm{C} 2=\mathrm{NN}=\mathrm{C} 3 \mathrm{C}(\mathrm{OCCC} 4=\mathrm{CC}=\mathrm{CC}=\mathrm{C} 4)= \\
\mathrm{NC}=\mathrm{CN} 32)=\mathrm{CC}(\mathrm{C}(\mathrm{C})(\mathrm{C}) \mathrm{C})=\mathrm{C} 1\end{array}$ \\
\hline $47 e$ & MK171b & $\begin{array}{l}\mathrm{C} 1(\mathrm{OCCC} 2=\mathrm{CC}=\mathrm{CC}=\mathrm{C} 2)=\mathrm{NC}=\mathrm{CN} 3 \mathrm{C} 1=\mathrm{NN}=\mathrm{C} 3 \mathrm{C} 4=\mathrm{C}(\mathrm{C} \\
=\mathrm{CC}=\mathrm{C} 5) \mathrm{C} 5=\mathrm{CC} 6=\mathrm{C} 4 \mathrm{C}=\mathrm{CC}=\mathrm{C} 6\end{array}$ \\
\hline $47 f$ & MK182b & $\begin{array}{l}\mathrm{COC}(\mathrm{C}=\mathrm{CC}=\mathrm{C} 1)=\mathrm{C} 1 \mathrm{C} 2=\mathrm{NN}=\mathrm{C} 3 \mathrm{C}(\mathrm{OCCC} 4=\mathrm{CC}=\mathrm{CC}=\mathrm{C} \\
4)=\mathrm{NC}=\mathrm{CN} 32\end{array}$ \\
\hline $48 a$ & MK120-5 & $\operatorname{COC}(\mathrm{C}=\mathrm{C} 1)=\mathrm{CC}=\mathrm{C} 1 \mathrm{C} 2=\mathrm{NN}=\mathrm{C} 3 \mathrm{C}(\mathrm{O})=\mathrm{NC}=\mathrm{CN} 32$ \\
\hline 48b & MK184c & $\begin{array}{l}\mathrm{OC} 1=\mathrm{NC}=\mathrm{CN} 2 \mathrm{C} 1=\mathrm{NN}=\mathrm{C} 2 \mathrm{C} 3=\mathrm{CC}=\mathrm{C}([\mathrm{N}+]([\mathrm{O}- \\
])=\mathrm{O}) \mathrm{C}=\mathrm{C} 3\end{array}$ \\
\hline 48c & MK182c & $\mathrm{OC} 1=\mathrm{NC}=\mathrm{CN} 2 \mathrm{C} 1=\mathrm{NN}=\mathrm{C} 2 \mathrm{C} 3=\mathrm{C}(\mathrm{OC}) \mathrm{C}=\mathrm{CC}=\mathrm{C} 3$ \\
\hline 48d & MK186c & $\begin{array}{l}\mathrm{OC} 1=\mathrm{NC}=\mathrm{CN} 2 \mathrm{C} 1=\mathrm{NN}=\mathrm{C} 2 \mathrm{C} 3=\mathrm{C}([\mathrm{N}+]([\mathrm{O}- \\
])=\mathrm{O}) \mathrm{C}=\mathrm{CC}=\mathrm{C} 3\end{array}$ \\
\hline 49 & MK128c & $\mathrm{COC}(\mathrm{C}=\mathrm{C} 1)=\mathrm{CC}=\mathrm{C} 1 \mathrm{C} 2=\mathrm{NN}=\mathrm{C} 3 \mathrm{C}=\mathrm{NC}=\mathrm{CN} 32$ \\
\hline 50 & MK128d & $\begin{array}{l}\mathrm{COC}(\mathrm{C}=\mathrm{C} 1)=\mathrm{CC}=\mathrm{C} 1 \mathrm{C} 2=\mathrm{NN}=\mathrm{C}(\mathrm{N} 2) \mathrm{C} 3=\mathrm{NC}=\mathrm{CN} 3 \mathrm{CCC} 4 \\
=\mathrm{CC}=\mathrm{CC}=\mathrm{C} 4\end{array}$ \\
\hline 55 & MK026a & $\mathrm{C} 12=\mathrm{NN}=\mathrm{CN} 1 \mathrm{C}(\mathrm{N} 3 \mathrm{CCCCC} 3)=\mathrm{CN}=\mathrm{C} 2$ \\
\hline 56 & MK026b & $\mathrm{C} 1(\mathrm{~N} 2 \mathrm{CCCCC} 2)=\mathrm{NC}=\mathrm{CN} 3 \mathrm{C} 1=\mathrm{NN}=\mathrm{C} 3$ \\
\hline 57 & MK026-7a & {$[2 \mathrm{H}] \mathrm{C} 1=\mathrm{NN}=\mathrm{C} 2 \mathrm{C}=\mathrm{NC}=\mathrm{C}(\mathrm{N} 3 \mathrm{CCCCC} 3) \mathrm{N} 21$} \\
\hline 58 & MK026-7b & {$[2 \mathrm{H}] \mathrm{C} 1=\mathrm{NN}=\mathrm{C} 2 \mathrm{C}(\mathrm{N} 3 \mathrm{CCCCC} 3)=\mathrm{NC}=\mathrm{C}([2 \mathrm{H}]) \mathrm{N} 21$} \\
\hline 59 & MK002-10 & $\mathrm{ClC} 1=\mathrm{CN}=\mathrm{CC} 2=\mathrm{NN}=\mathrm{C}([2 \mathrm{H}]) \mathrm{N} 21$ \\
\hline 60 & MK026-9 & {$[2 \mathrm{H}] \mathrm{C} 1=\mathrm{NN}=\mathrm{C} 2 \mathrm{C}(\mathrm{N} 3 \mathrm{CCCCC} 3)=\mathrm{NC}=\mathrm{CN} 21$} \\
\hline
\end{tabular}




\section{Compound} structure and number<smiles>COc1ccc(-c2nnc3cncc(SCCc4ccccc4)n23)cc1</smiles>

$46 d$

\begin{tabular}{|l|l|r|r|}
\hline & & & \\
DiscoveRx Gene Symbol & $\begin{array}{l}\text { Entrez Gene } \\
\text { Symbol }\end{array}$ & $\begin{array}{r}\text { Percent } \\
\text { Control }\end{array}$ & \multicolumn{1}{l|}{ C (nM) } \\
\hline AURKC & AURKC & 0 & 1000 \\
\hline BIKE & BMP2K & 0 & 1000 \\
\hline BMX & BMX & 0 & 1000 \\
\hline FLT1 & FLT1 & 0 & 1000 \\
\hline FLT3(ITD) & FLT3 & 0 & 1000 \\
\hline PIK3CD & PIK3CD & 0 & 1000 \\
\hline RIOK3 & RIOK3 & 0 & 1000 \\
\hline RIOK1 & RIOK1 & 11 & 1000 \\
\hline FLT3(ITD,F691L) & FLT3 & 39 & 1000 \\
\hline TSSK1B & TSSK1B & 46 & 1000 \\
\hline TNK1 & TNK1 & 47 & 1000 \\
\hline EGFR(L861Q) & EGFR & 50 & 1000 \\
\hline IKK-alpha & CHUK & 53 & 1000 \\
\hline BRAF & BRAF & 54 & 1000 \\
\hline EGFR(L747-T751del,Sins) & EGFR & 56 & 1000 \\
\hline EPHA2 & EPHA2 & 61 & 1000 \\
\hline MKNK1 & MKNK1 & 64 & 1000 \\
\hline ERBB2 & ERBB2 & 65 & 1000 \\
\hline ULK3 & ULK3 & 65 & 1000 \\
\hline PRKD3 & PRKD3 & 67 & 1000 \\
\hline EGFR(L858R,T790M) & EGFR & 70 & 1000 \\
\hline KIT(D816H) & KIT & 70 & 1000 \\
\hline DCAMKL1 & DCLK1 & 71 & 1000 \\
\hline & & & \\
\hline
\end{tabular}<smiles>COc1ccc(-c2nnc3c(SCCc4ccccc4)nccn23)cc1</smiles>

$47 b$

\begin{tabular}{|l|l|r|r|}
\hline & & & \\
& $\begin{array}{l}\text { Entrez } \\
\text { Gene } \\
\text { DiscoveRx Gene Symbol }\end{array}$ & $\begin{array}{r}\text { Percent } \\
\text { Control }\end{array}$ & C (nM) \\
\hline RIOK1 & RIOK1 & 16 & 1000 \\
\hline MST2 & STK3 & 42 & 1000 \\
\hline TSSK1B & TSSK1B & 42 & 1000 \\
\hline EGFR(L861Q) & EGFR & 48 & 1000 \\
\hline BRAF & BRAF & 51 & 1000 \\
\hline FLT3(ITD,F691L) & FLT3 & 54 & 1000 \\
\hline IKK-alpha & CHUK & 57 & 1000 \\
\hline ERBB2 & ERBB2 & 60 & 1000 \\
\hline RAF1 & RAF1 & 61 & 1000 \\
\hline SBK1 & SBK1 & 65 & 1000 \\
\hline KIT(D816H) & KIT & 67 & 1000 \\
\hline PAK2 & PAK2 & 67 & 1000 \\
\hline JAK2(JH1domain-catalytic) & JAK2 & 68 & 1000 \\
\hline EGFR(L747-T751del,Sins) & EGFR & 69 & 1000 \\
\hline PIK3CA(H1047Y) & PIK3CA & 69 & 1000 \\
\hline PIP5K2C & PIP4K2C & 69 & 1000 \\
\hline PRKCE & PRKCE & 69 & 1000 \\
\hline TYRO3 & TYRO3 & 69 & 1000 \\
\hline MYYK4 & MYLK4 & 71 & 1000 \\
\hline PKMYT1 & PKMYT1 & 71 & 1000 \\
\hline TYK2(JH2domain-pseudokinas & TYK2 & 71 & 1000 \\
\hline MEK5 & MAP2K5 & 72 & 1000 \\
\hline TNK1 & TNK1 & 72 & 1000 \\
\hline & & & \\
\hline
\end{tabular}




\begin{tabular}{|c|c|c|c|}
\hline DLK & MAР3К12 & 71 & 1000 \\
\hline EGFR(S752-1759del) & EGFR & 72 & 1000 \\
\hline AURKB & AURKB & 73 & 1000 \\
\hline DYRK1B & DYRK1B & 73 & 1000 \\
\hline FLT3(ITD,D835V) & FLT3 & 74 & 1000 \\
\hline SRMS & SRMS & 74 & 1000 \\
\hline GCN2(Kin.Dom.2,S808G) & EIF2AK4 & 76 & 1000 \\
\hline HPK1 & MAP4K1 & 76 & 1000 \\
\hline JNK1 & MAPK8 & 76 & 1000 \\
\hline MINK & MINK1 & 76 & 1000 \\
\hline NDR1 & STK38 & 76 & 1000 \\
\hline PIK3CA(E545K) & PIK3CA & 76 & 1000 \\
\hline PIK4CB & PI4KB & 76 & 1000 \\
\hline CDK8 & CDK8 & 77 & 1000 \\
\hline DDR2 & DDR2 & 77 & 1000 \\
\hline MAP3K4 & MAP3K4 & 77 & 1000 \\
\hline PLK2 & PLK2 & 77 & 1000 \\
\hline CDK4-cyclinD1 & CDK4 & 78 & 1000 \\
\hline CDKL3 & CDKL3 & 78 & 1000 \\
\hline SRPK2 & SRPK2 & 78 & 1000 \\
\hline BRSK1 & BRSK1 & 79 & 1000 \\
\hline ERN1 & ERN1 & 79 & 1000 \\
\hline FYN & FYN & 79 & 1000 \\
\hline BMPR2 & BMPR2 & 80 & 1000 \\
\hline FGFR2 & FGFR2 & 80 & 1000 \\
\hline FLT3(R834Q) & FLT3 & 80 & 1000 \\
\hline ICK & ICK & 80 & 1000 \\
\hline PIK3CA(H1047Y) & PIK3CA & 80 & 1000 \\
\hline ROCK2 & ROCK2 & 80 & 1000 \\
\hline RPS6KA4(Kin.Dom.2-C-term & RPS6KA4 & 80 & 1000 \\
\hline VPS34 & РIK3С3 & 80 & 1000 \\
\hline ABL1-nonphosphorylated & ABL1 & 81 & 1000 \\
\hline ALK & ALK & 81 & 1000 \\
\hline JNK2 & MAPK9 & 81 & 1000 \\
\hline JNK3 & MAPK10 & 81 & 1000 \\
\hline MAР3К15 & MАР3К15 & 81 & 1000 \\
\hline MEK5 & MAP2K5 & 81 & 1000 \\
\hline MST2 & STK3 & 81 & 1000 \\
\hline NIK & МАРЗК14 & 81 & 1000 \\
\hline RIPK5 & DSTYK & 81 & 1000 \\
\hline SYK & SYK & 81 & 1000 \\
\hline TAOK2 & TAOK2 & 81 & 1000 \\
\hline TRPM6 & TRPM6 & 81 & 1000 \\
\hline
\end{tabular}

\begin{tabular}{|c|c|c|c|}
\hline ULK3 & ULK3 & 72 & 1000 \\
\hline ERBB3 & ERBB3 & 73 & 1000 \\
\hline PIK3CA(E545K) & PIK3CA & 73 & 1000 \\
\hline STK33 & STK33 & 73 & 1000 \\
\hline TGFBR1 & TGFBR1 & 73 & 1000 \\
\hline AURKB & AURKB & 75 & 1000 \\
\hline BRSK1 & BRSK1 & 75 & 1000 \\
\hline TNNI3K & TNNI3K & 75 & 1000 \\
\hline YSK4 & MAP3К19 & 76 & 1000 \\
\hline CAMK2D & CAMK2D & 77 & 1000 \\
\hline PIP5K1C & PIP5K1C & 77 & 1000 \\
\hline SRMS & SRMS & 77 & 1000 \\
\hline ABL1-nonphosphorylated & ABL1 & 78 & 1000 \\
\hline PIKFYVE & PIKFYVE & 79 & 1000 \\
\hline ASK2 & MAP3K6 & 80 & 1000 \\
\hline MAK & MAK & 80 & 1000 \\
\hline MKNK1 & MKNK1 & 80 & 1000 \\
\hline DCAMKL1 & DCLK1 & 81 & 1000 \\
\hline MARK1 & MARK1 & 81 & 1000 \\
\hline NDR1 & STK38 & 81 & 1000 \\
\hline KIT(L576P) & KIT & 82 & 1000 \\
\hline MAP3K2 & MAР3К2 & 82 & 1000 \\
\hline RSK2(Kin.Dom.2-C-terminal) & RPS6KA3 & 82 & 1000 \\
\hline ANKK1 & ANKK1 & 83 & 1000 \\
\hline DDR2 & DDR2 & 83 & 1000 \\
\hline PRKD3 & PRKD3 & 83 & 1000 \\
\hline RIPK4 & RIPK4 & 83 & 1000 \\
\hline CASK & CASK & 84 & 1000 \\
\hline FLT3(ITD,D835V) & FLT3 & 84 & 1000 \\
\hline MINK & MINK1 & 84 & 1000 \\
\hline PIK3CA(E542K) & PIK3CA & 84 & 1000 \\
\hline PIK4CB & PI4KB & 84 & 1000 \\
\hline PLK3 & PLK3 & 84 & 1000 \\
\hline RSK1(Kin.Dom.1-N-terminal) & RPS6KA1 & 84 & 1000 \\
\hline TAOK2 & TAOK2 & 84 & 1000 \\
\hline WNK2 & WNK2 & 84 & 1000 \\
\hline ABL1(M351T)-phosphorylatec & ABL1 & 85 & 1000 \\
\hline BMX & BMX & 85 & 1000 \\
\hline CSF1R & CSF1R & 85 & 1000 \\
\hline PIK3CA(1800L) & PIK3CA & 85 & 1000 \\
\hline SNRK & SNRK & 85 & 1000 \\
\hline ABL1(T315I)-phosphorylated & ABL1 & 86 & 1000 \\
\hline FLT3(R834Q) & FLT3 & 86 & 1000 \\
\hline
\end{tabular}




\begin{tabular}{|c|c|c|c|}
\hline CDC2L5 & CDK13 & 82 & 1000 \\
\hline HIPK4 & HIPK4 & 82 & 1000 \\
\hline MAP3К1 & MAP3K1 & 82 & 1000 \\
\hline MEK1 & MAP2K1 & 82 & 1000 \\
\hline OSR1 & OXSR1 & 82 & 1000 \\
\hline PLK3 & PLK3 & 82 & 1000 \\
\hline FLT3(K663Q) & FLT3 & 83 & 1000 \\
\hline MAP4K2 & MAP4K2 & 83 & 1000 \\
\hline PFCDPK1(P.falciparum) & CDPK1 & 83 & 1000 \\
\hline ULK1 & ULK1 & 83 & 1000 \\
\hline ANKK1 & ANKK1 & 84 & 1000 \\
\hline MAP3К2 & MAP3K2 & 84 & 1000 \\
\hline MEK3 & MAP2K3 & 84 & 1000 \\
\hline MEK4 & MAP2K4 & 84 & 1000 \\
\hline PIK3CA(E542K) & PIKЗСА & 84 & 1000 \\
\hline PIK3CA(1800L) & PIK3CA & 84 & 1000 \\
\hline ROCK1 & ROCK1 & 84 & 1000 \\
\hline TYRO3 & TYRO3 & 84 & 1000 \\
\hline WNK2 & WNK2 & 84 & 1000 \\
\hline YSK4 & MAРЗК19 & 84 & 1000 \\
\hline ZAP70 & ZAP70 & 84 & 1000 \\
\hline BLK & BLK & 85 & 1000 \\
\hline FAK & PTK2 & 85 & 1000 \\
\hline FLT3(D835V) & FLT3 & 85 & 1000 \\
\hline MAK & MAK & 85 & 1000 \\
\hline MAP4K3 & MAP4K3 & 85 & 1000 \\
\hline PIM2 & PIM2 & 85 & 1000 \\
\hline SGK3 & SGK3 & 85 & 1000 \\
\hline EGFR(T790M) & EGFR & 86 & 1000 \\
\hline MEK2 & MAP2K2 & 86 & 1000 \\
\hline PIK3CA(H1047L) & PIK3CA & 86 & 1000 \\
\hline PRKG2 & PRKG2 & 86 & 1000 \\
\hline RSK2(Kin.Dom.2-C-terminal & RPS6KA3 & 86 & 1000 \\
\hline ADCK3 & CABC1 & 87 & 1000 \\
\hline BUB1 & BUB1 & 87 & 1000 \\
\hline CDK4 & CDK4 & 87 & 1000 \\
\hline CDKL1 & CDKL1 & 87 & 1000 \\
\hline CDKL5 & CDKL5 & 87 & 1000 \\
\hline JAK3(JH1domain-catalytic) & JAK3 & 87 & 1000 \\
\hline MARK2 & MARK2 & 87 & 1000 \\
\hline TAOK1 & TAOK1 & 87 & 1000 \\
\hline TAOK3 & TAOK3 & 87 & 1000 \\
\hline ULK2 & ULK2 & 87 & 1000 \\
\hline
\end{tabular}

\begin{tabular}{|c|c|c|c|}
\hline PIP5K1A & PIP5K1A & 86 & 1000 \\
\hline QSK & KIAA0999 & 86 & 1000 \\
\hline RIPK5 & DSTYK & 86 & 1000 \\
\hline ZAP70 & ZAP70 & 86 & 1000 \\
\hline FLT3(D835V) & FLT3 & 87 & 1000 \\
\hline HASPIN & GSG2 & 87 & 1000 \\
\hline IKK-beta & IKBKB & 87 & 1000 \\
\hline JNK2 & MAPK9 & 87 & 1000 \\
\hline PHKG2 & PHKG2 & 87 & 1000 \\
\hline PLK2 & PLK2 & 87 & 1000 \\
\hline RSK4(Kin.Dom.2-C-terminal) & RPS6KA6 & 87 & 1000 \\
\hline BRSK2 & BRSK2 & 88 & 1000 \\
\hline GRK1 & GRK1 & 88 & 1000 \\
\hline JNK3 & MAPK10 & 88 & 1000 \\
\hline MAP3K15 & MAP3K15 & 88 & 1000 \\
\hline MEK3 & MAP2K3 & 88 & 1000 \\
\hline SGK3 & SGK3 & 88 & 1000 \\
\hline SYK & SYK & 88 & 1000 \\
\hline ULK1 & ULK1 & 88 & 1000 \\
\hline MARK2 & MARK2 & 89 & 1000 \\
\hline NIM1 & MGC4210 & 89 & 1000 \\
\hline PAK7 & PAK7 & 89 & 1000 \\
\hline DLK & MAP3K12 & 90 & 1000 \\
\hline IRAK4 & IRAK4 & 90 & 1000 \\
\hline PIM2 & PIM2 & 90 & 1000 \\
\hline SGK & SGK1 & 90 & 1000 \\
\hline TAOK1 & TAOK1 & 90 & 1000 \\
\hline CDK8 & CDK8 & 91 & 1000 \\
\hline EGFR(L858R,T790M) & EGFR & 91 & 1000 \\
\hline FYN & FYN & 91 & 1000 \\
\hline MAP3K4 & MАРЗК4 & 91 & 1000 \\
\hline MEK1 & MAP2K1 & 91 & 1000 \\
\hline NEK10 & NEK10 & 91 & 1000 \\
\hline PIK3CD & PIK3CD & 91 & 1000 \\
\hline PRKD2 & PRKD2 & 91 & 1000 \\
\hline CAMK1G & CAMK1G & 92 & 1000 \\
\hline DYRK2 & DYRK2 & 92 & 1000 \\
\hline ICK & ICK & 92 & 1000 \\
\hline KIT(V559D,T670I) & KIT & 92 & 1000 \\
\hline MAPKAPK5 & MAPKAPK & 92 & 1000 \\
\hline RPS6KA4(Kin.Dom.2-C-termin & RPS6KA4 & 92 & 1000 \\
\hline TLK1 & TLK1 & 92 & 1000 \\
\hline KIT(V559D) & KIT & 93 & 1000 \\
\hline
\end{tabular}




\begin{tabular}{|c|c|c|c|}
\hline DMPK & DMPK & 88 & 1000 \\
\hline DYRK1A & DYRK1A & 88 & 1000 \\
\hline EGFR(G719C) & EGFR & 88 & 1000 \\
\hline ERBB3 & ERBB3 & 88 & 1000 \\
\hline IRAK4 & IRAK4 & 88 & 1000 \\
\hline PIK3C2B & PIK3C2B & 88 & 1000 \\
\hline PKN1 & PKN1 & 88 & 1000 \\
\hline RPS6KA5(Kin.Dom.2-C-term & RPS6KA5 & 88 & 1000 \\
\hline SBK1 & SBK1 & 88 & 1000 \\
\hline SIK2 & SIK2 & 88 & 1000 \\
\hline TYK2(JH2domain-pseudokir & TTYK2 & 88 & 1000 \\
\hline ABL1(H396P)-nonphosphor & ABL1 & 89 & 1000 \\
\hline ABL1(M351T)-phosphorylat & ABBL1 & 89 & 1000 \\
\hline CASK & CASK & 89 & 1000 \\
\hline FLT3-autoinhibited & FLT3 & 89 & 1000 \\
\hline KIT(V559D) & KIT & 89 & 1000 \\
\hline NEK11 & NEK11 & 89 & 1000 \\
\hline S6K1 & RPS6KB1 & 89 & 1000 \\
\hline SRPK1 & SRPK1 & 89 & 1000 \\
\hline TBK1 & TBK1 & 89 & 1000 \\
\hline TNNI3K & TNNI3K & 89 & 1000 \\
\hline BTK & BTK & 90 & 1000 \\
\hline EGFR(L747-E749del, A750P & EGFR & 90 & 1000 \\
\hline FGFR3 & FGFR3 & 90 & 1000 \\
\hline MAPKAPK5 & MAPKAPK5 & 90 & 1000 \\
\hline MKK7 & MAP2K7 & 90 & 1000 \\
\hline SRC & SRC & 90 & 1000 \\
\hline AKT1 & AKT1 & 91 & 1000 \\
\hline CHEK2 & CHEK2 & 91 & 1000 \\
\hline CSF1R-autoinhibited & CSF1R & 91 & 1000 \\
\hline NIM1 & MGC42105 & 91 & 1000 \\
\hline PIP5K1C & PIP5K1C & 91 & 1000 \\
\hline SNARK & NUAK2 & 91 & 1000 \\
\hline TRKC & NTRK3 & 91 & 1000 \\
\hline ABL1-phosphorylated & ABL1 & 92 & 1000 \\
\hline ASK1 & MAP3K5 & 92 & 1000 \\
\hline BRSK2 & BRSK2 & 92 & 1000 \\
\hline GRK1 & GRK1 & 92 & 1000 \\
\hline HIPK3 & HIPK3 & 92 & 1000 \\
\hline IRAK1 & IRAK1 & 92 & 1000 \\
\hline MST4 & MST4 & 92 & 1000 \\
\hline NDR2 & STK38L & 92 & 1000 \\
\hline PAK2 & PAK2 & 92 & 1000 \\
\hline
\end{tabular}

\begin{tabular}{|c|c|c|c|}
\hline MKK7 & MAP2K7 & 93 & 1000 \\
\hline NDR2 & STK38L & 93 & 1000 \\
\hline PIK3CA(E545A) & PIK3CA & 93 & 1000 \\
\hline RIPK2 & RIPK2 & 93 & 1000 \\
\hline TBK1 & TBK1 & 93 & 1000 \\
\hline ABL1(Q252H)-nonphosphoryl & ABL1 & 94 & 1000 \\
\hline AKT1 & AKT1 & 94 & 1000 \\
\hline BMPR2 & BMPR2 & 94 & 1000 \\
\hline BRAF(V600E) & BRAF & 94 & 1000 \\
\hline CDK4-cyclinD1 & CDK4 & 94 & 1000 \\
\hline DMPK & DMPK & 94 & 1000 \\
\hline DYRK1A & DYRK1A & 94 & 1000 \\
\hline MST3 & STK24 & 94 & 1000 \\
\hline MST4 & MST4 & 94 & 1000 \\
\hline SNARK & NUAK2 & 94 & 1000 \\
\hline ABL1-phosphorylated & ABL1 & 95 & 1000 \\
\hline AKT2 & AKT2 & 95 & 1000 \\
\hline CLK1 & CLK1 & 95 & 1000 \\
\hline CLK2 & CLK2 & 95 & 1000 \\
\hline DAPK3 & DAPK3 & 95 & 1000 \\
\hline LRRK2 & LRRK2 & 95 & 1000 \\
\hline PFTAIRE2 & CDK15 & 95 & 1000 \\
\hline TAOK3 & TAOK3 & 95 & 1000 \\
\hline TRKB & NTRK2 & 95 & 1000 \\
\hline VRK2 & VRK2 & 95 & 1000 \\
\hline BTK & BTK & 96 & 1000 \\
\hline САМКК2 & CAMKK2 & 96 & 1000 \\
\hline EGFR(E746-A750del) & EGFR & 96 & 1000 \\
\hline $\operatorname{EGFR(G719C)}$ & EGFR & 96 & 1000 \\
\hline PRKR & EIF2AK2 & 96 & 1000 \\
\hline ROCK1 & ROCK1 & 96 & 1000 \\
\hline TRKA & NTRK1 & 96 & 1000 \\
\hline WEE1 & WEE1 & 96 & 1000 \\
\hline WEE2 & WEE2 & 96 & 1000 \\
\hline CDKL1 & CDKL1 & 97 & 1000 \\
\hline CLK4 & CLK4 & 97 & 1000 \\
\hline EGFR(S752-1759del) & EGFR & 97 & 1000 \\
\hline EPHA4 & EPHA4 & 97 & 1000 \\
\hline FLT3-autoinhibited & FLT3 & 97 & 1000 \\
\hline JNK1 & MAPK8 & 97 & 1000 \\
\hline MAP4K3 & MAP4K3 & 97 & 1000 \\
\hline MARK4 & MARK4 & 97 & 1000 \\
\hline MEK2 & MAP2K2 & 97 & 1000 \\
\hline
\end{tabular}




\begin{tabular}{|c|c|c|c|}
\hline PRKCE & PRKCE & 92 & 1000 \\
\hline TNIK & TNIK & 92 & 1000 \\
\hline TYK2(JH1domain-catalytic) & TYK2 & 92 & 1000 \\
\hline IKK-beta & IKBKB & 93 & 1000 \\
\hline MYLK & MYLK & 93 & 1000 \\
\hline PCTK1 & CDK16 & 93 & 1000 \\
\hline VRK2 & VRK2 & 93 & 1000 \\
\hline YANK1 & STK32A & 93 & 1000 \\
\hline FLT3(N841I) & FLT3 & 94 & 1000 \\
\hline KIT(L576P) & KIT & 94 & 1000 \\
\hline LCK & LCK & 94 & 1000 \\
\hline PDGFRB & PDGFRB & 94 & 1000 \\
\hline PIK3CA(E545A) & PIK3CA & 94 & 1000 \\
\hline PIM1 & PIM1 & 94 & 1000 \\
\hline PKMYT1 & PKMYT1 & 94 & 1000 \\
\hline PRKCl & PRKCl & 94 & 1000 \\
\hline STK33 & STK33 & 94 & 1000 \\
\hline TXK & TXK & 94 & 1000 \\
\hline EGFR(G719S) & EGFR & 95 & 1000 \\
\hline EIF2AK1 & EIF2AK1 & 95 & 1000 \\
\hline FLT3(D835Y) & FLT3 & 95 & 1000 \\
\hline HASPIN & GSG2 & 95 & 1000 \\
\hline LIMK1 & LIMK1 & 95 & 1000 \\
\hline MLK2 & MAP3К10 & 95 & 1000 \\
\hline RIPK4 & RIPK4 & 95 & 1000 \\
\hline SLK & SLK & 95 & 1000 \\
\hline SNRK & SNRK & 95 & 1000 \\
\hline ABL1(F317I)-phosphorylate & $A B L 1$ & 96 & 1000 \\
\hline ABL1(T315I)-phosphorylate & $A B L 1$ & 96 & 1000 \\
\hline BMPR1B & BMPR1B & 96 & 1000 \\
\hline BRAF(V600E) & BRAF & 96 & 1000 \\
\hline CSNK1A1 & CSNK1A1 & 96 & 1000 \\
\hline KIT(A829P) & KIT & 96 & 1000 \\
\hline LRRK2 & LRRK2 & 96 & 1000 \\
\hline PIK3CA(C42OR) & PIK3CA & 96 & 1000 \\
\hline PIM3 & PIM3 & 96 & 1000 \\
\hline PRKD2 & PRKD2 & 96 & 1000 \\
\hline EPHB2 & EPHB2 & 97 & 1000 \\
\hline GRK3 & ADRBK2 & 97 & 1000 \\
\hline GSK3B & GSK3B & 97 & 1000 \\
\hline HIPK1 & HIPK1 & 97 & 1000 \\
\hline LYN & LYN & 97 & 1000 \\
\hline MEK6 & MAP2K6 & 97 & 1000 \\
\hline
\end{tabular}

\begin{tabular}{|c|c|c|c|}
\hline MEK6 & MAP2K6 & 97 & 1000 \\
\hline PIK3CA(Q546K) & PIK3СA & 97 & 1000 \\
\hline RSK4(Kin.Dom.1-N-terminal) & RPS6KA6 & 97 & 1000 \\
\hline TNIK & TNIK & 97 & 1000 \\
\hline YANK2 & STK32B & 97 & 1000 \\
\hline YANK3 & STK32C & 97 & 1000 \\
\hline AMPK-alpha2 & PRKAA2 & 98 & 1000 \\
\hline GCN2(Kin.Dom.2,S808G) & EIF2AK4 & 98 & 1000 \\
\hline GSK3B & GSK3B & 98 & 1000 \\
\hline MAP3K1 & MAP3K1 & 98 & 1000 \\
\hline MAP4K2 & MAP4K2 & 98 & 1000 \\
\hline MTOR & MTOR & 98 & 1000 \\
\hline OSR1 & OXSR1 & 98 & 1000 \\
\hline PAK1 & PAK1 & 98 & 1000 \\
\hline PFCDPK1(P.falciparum) & CDPK1 & 98 & 1000 \\
\hline RIPK1 & RIPK1 & 98 & 1000 \\
\hline RSK2(Kin.Dom.1-N-terminal) & RPS6KA3 & 98 & 1000 \\
\hline RSK3(Kin.Dom.2-C-terminal) & RPS6KA2 & 98 & 1000 \\
\hline TRPM6 & TRPM6 & 98 & 1000 \\
\hline CDKL2 & CDKL2 & 99 & 1000 \\
\hline DAPK2 & DAPK2 & 99 & 1000 \\
\hline PDGFRB & PDGFRB & 99 & 1000 \\
\hline RPS6KA5(Kin.Dom.2-C-termin & RPS6KA5 & 99 & 1000 \\
\hline RSK1(Kin.Dom.2-C-terminal) & RPS6KA1 & 99 & 1000 \\
\hline S6K1 & RPS6KB1 & 99 & 1000 \\
\hline TAK1 & MAP3K7 & 99 & 1000 \\
\hline AAK1 & AAK1 & 100 & 1000 \\
\hline ABL1(E255K)-phosphorylated & ABL1 & 100 & 1000 \\
\hline ABL1(F317I)-nonphosphorylat & ABL1 & 100 & 1000 \\
\hline ABL1(F317I)-phosphorylated & ABL1 & 100 & 1000 \\
\hline ABL1(F317L)-nonphosphorylat & ABL1 & 100 & 1000 \\
\hline ABL1(F317L)-phosphorylated & ABL1 & 100 & 1000 \\
\hline ABL1(H396P)-nonphosphoryla & ABL1 & 100 & 1000 \\
\hline ABL1(H396P)-phosphorylated & ABL1 & 100 & 1000 \\
\hline ABL1(Q252H)-phosphorylated & ABL1 & 100 & 1000 \\
\hline ABL1(T315I)-nonphosphorylat & ABL1 & 100 & 1000 \\
\hline ABL1(Y253F)-phosphorylated & ABL1 & 100 & 1000 \\
\hline ABL2 & ABL2 & 100 & 1000 \\
\hline ACVR1 & ACVR1 & 100 & 1000 \\
\hline ACVR1B & ACVR1B & 100 & 1000 \\
\hline ACVR2A & ACVR2A & 100 & 1000 \\
\hline ACVR2B & ACVR2B & 100 & 1000 \\
\hline ACVRL1 & ACVRL1 & 100 & 1000 \\
\hline
\end{tabular}




\begin{tabular}{|c|c|c|c|}
\hline MYLK2 & MYLK2 & 97 & 1000 \\
\hline NEK5 & NEK5 & 97 & 1000 \\
\hline TTK & TTK & 97 & 1000 \\
\hline VEGFR2 & KDR & 97 & 1000 \\
\hline CAMKK2 & САМКК2 & 98 & 1000 \\
\hline CDK7 & CDK7 & 98 & 1000 \\
\hline GRK7 & GRK7 & 98 & 1000 \\
\hline KIT & KIT & 98 & 1000 \\
\hline KIT(D816V) & KIT & 98 & 1000 \\
\hline RSK1(Kin.Dom.1-N-termina & RPS6KA1 & 98 & 1000 \\
\hline SGK & SGK1 & 98 & 1000 \\
\hline SIK & SIK1 & 98 & 1000 \\
\hline WNK4 & WNK4 & 98 & 1000 \\
\hline CAMK2D & CAMK2D & 99 & 1000 \\
\hline DMPK2 & CDC42BPG & 99 & 1000 \\
\hline EGFR(E746-A750del) & EGFR & 99 & 1000 \\
\hline HIPK2 & HIPK2 & 99 & 1000 \\
\hline KIT(V559D,V654A) & KIT & 99 & 1000 \\
\hline LZK & MAР3К13 & 99 & 1000 \\
\hline PKAC-alpha & PRKACA & 99 & 1000 \\
\hline QSK & KIAA0999 & 99 & 1000 \\
\hline RIOK2 & RIOK2 & 99 & 1000 \\
\hline ROS1 & ROS1 & 99 & 1000 \\
\hline SGK2 & SGK2 & 99 & 1000 \\
\hline TAK1 & MAP3K7 & 99 & 1000 \\
\hline WNK1 & WNK1 & 99 & 1000 \\
\hline AAK1 & AAK1 & 100 & 1000 \\
\hline ABL1(E255K)-phosphorylate & ABL1 & 100 & 1000 \\
\hline ABL1(F317l)-nonphosphory & ABL1 & 100 & 1000 \\
\hline ABL1(F317L)-nonphosphory & ABL1 & 100 & 1000 \\
\hline ABL1(F317L)-phosphorylate & ABL1 & 100 & 1000 \\
\hline ABL1(H396P)-phosphorylat & ABL1 & 100 & 1000 \\
\hline ABL1(Q252H)-nonphosphor & ABL1 & 100 & 1000 \\
\hline ABL1(Q252H)-phosphorylat & ABL1 & 100 & 1000 \\
\hline ABL1(T315I)-nonphosphory & ABL1 & 100 & 1000 \\
\hline ABL1(Y253F)-phosphorylate & ABL1 & 100 & 1000 \\
\hline ABL2 & ABL2 & 100 & 1000 \\
\hline ACVR1 & ACVR1 & 100 & 1000 \\
\hline ACVR1B & ACVR1B & 100 & 1000 \\
\hline ACVR2A & ACVR2A & 100 & 1000 \\
\hline ACVR2B & ACVR2B & 100 & 1000 \\
\hline ACVRL1 & ACVRL1 & 100 & 1000 \\
\hline ADCK4 & ADCK4 & 100 & 1000 \\
\hline
\end{tabular}

\begin{tabular}{|c|c|c|c|}
\hline ADCK3 & CABC1 & 100 & 1000 \\
\hline ADCK4 & ADCK4 & 100 & 1000 \\
\hline AKT3 & АКT3 & 100 & 1000 \\
\hline ALK & ALK & 100 & 1000 \\
\hline ALK(C1156Y) & ALK & 100 & 1000 \\
\hline ALK(L1196M) & ALK & 100 & 1000 \\
\hline AMPK-alpha1 & PRKAA1 & 100 & 1000 \\
\hline ARK5 & NUAK1 & 100 & 1000 \\
\hline ASK1 & MAP3K5 & 100 & 1000 \\
\hline AURKA & AURKA & 100 & 1000 \\
\hline AURKC & AURKC & 100 & 1000 \\
\hline AXL & AXL & 100 & 1000 \\
\hline BIKE & BMP2K & 100 & 1000 \\
\hline BLK & BLK & 100 & 1000 \\
\hline BMPR1A & BMPR1A & 100 & 1000 \\
\hline BMPR1B & BMPR1B & 100 & 1000 \\
\hline BRK & PTK6 & 100 & 1000 \\
\hline BUB1 & BUB1 & 100 & 1000 \\
\hline CAMK1 & CAMK1 & 100 & 1000 \\
\hline CAMK1B & PNCK & 100 & 1000 \\
\hline CAMK1D & CAMK1D & 100 & 1000 \\
\hline CAMK2A & САМК2А & 100 & 1000 \\
\hline CAMK2B & CAMK2B & 100 & 1000 \\
\hline CAMK2G & CAMK2G & 100 & 1000 \\
\hline CAMK4 & CAMK4 & 100 & 1000 \\
\hline CAMKK1 & CAMKK1 & 100 & 1000 \\
\hline CDC2L1 & CDK11B & 100 & 1000 \\
\hline CDC2L2 & CDC2L2 & 100 & 1000 \\
\hline CDC2L5 & CDK13 & 100 & 1000 \\
\hline CDK11 & CDK19 & 100 & 1000 \\
\hline CDK2 & CDK2 & 100 & 1000 \\
\hline CDK3 & CDK3 & 100 & 1000 \\
\hline CDK4 & CDK4 & 100 & 1000 \\
\hline CDK4-cyclinD3 & CDK4 & 100 & 1000 \\
\hline CDK5 & CDK5 & 100 & 1000 \\
\hline CDK7 & CDK7 & 100 & 1000 \\
\hline CDK9 & CDK9 & 100 & 1000 \\
\hline CDKL3 & CDKL3 & 100 & 1000 \\
\hline CDKL5 & CDKL5 & 100 & 1000 \\
\hline CHEK1 & CHEK1 & 100 & 1000 \\
\hline CHEK2 & CHEK2 & 100 & 1000 \\
\hline CIT & CIT & 100 & 1000 \\
\hline CLK3 & CLK3 & 100 & 1000 \\
\hline
\end{tabular}




\begin{tabular}{|c|c|c|c|}
\hline AKT2 & AKT2 & 100 & 1000 \\
\hline AKT3 & AKT3 & 100 & 1000 \\
\hline ALK(C1156Y) & ALK & 100 & 1000 \\
\hline ALK(L1196M) & ALK & 100 & 1000 \\
\hline AMPK-alpha1 & PRKAA1 & 100 & 1000 \\
\hline AMPK-alpha2 & PRKAA2 & 100 & 1000 \\
\hline ARK5 & NUAK1 & 100 & 1000 \\
\hline ASK2 & МАРЗК6 & 100 & 1000 \\
\hline AURKA & AURKA & 100 & 1000 \\
\hline AXL & AXL & 100 & 1000 \\
\hline BMPR1A & BMPR1A & 100 & 1000 \\
\hline BRK & PTK6 & 100 & 1000 \\
\hline CAMK1 & CAMK1 & 100 & 1000 \\
\hline CAMK1B & PNCK & 100 & 1000 \\
\hline CAMK1D & CAMK1D & 100 & 1000 \\
\hline CAMK1G & CAMK1G & 100 & 1000 \\
\hline САMK2A & САMK2А & 100 & 1000 \\
\hline CAMK2B & САMK2B & 100 & 1000 \\
\hline CAMK2G & CAMK2G & 100 & 1000 \\
\hline CAMK4 & CAMK4 & 100 & 1000 \\
\hline CAMKK1 & CAMKK1 & 100 & 1000 \\
\hline CDC2L1 & CDK11B & 100 & 1000 \\
\hline CDC2L2 & CDC2L2 & 100 & 1000 \\
\hline CDK11 & CDK19 & 100 & 1000 \\
\hline CDK2 & CDK2 & 100 & 1000 \\
\hline CDK3 & CDK3 & 100 & 1000 \\
\hline CDK4-cyclinD3 & CDK4 & 100 & 1000 \\
\hline CDK5 & CDK5 & 100 & 1000 \\
\hline CDK9 & CDK9 & 100 & 1000 \\
\hline CDKL2 & CDKL2 & 100 & 1000 \\
\hline CHEK1 & CHEK1 & 100 & 1000 \\
\hline CIT & CIT & 100 & 1000 \\
\hline CLK1 & CLK1 & 100 & 1000 \\
\hline CLK2 & CLK2 & 100 & 1000 \\
\hline CLK3 & CLK3 & 100 & 1000 \\
\hline CLK4 & CLK4 & 100 & 1000 \\
\hline CSF1R & CSF1R & 100 & 1000 \\
\hline CSK & CSK & 100 & 1000 \\
\hline CSNK1A1L & CSNK1A1L & 100 & 1000 \\
\hline CSNK1D & CSNK1D & 100 & 1000 \\
\hline CSNK1E & CSNK1E & 100 & 1000 \\
\hline CSNK1G1 & CSNK1G1 & 100 & 1000 \\
\hline CSNK1G2 & CSNK1G2 & 100 & 1000 \\
\hline
\end{tabular}

\begin{tabular}{|c|c|c|c|}
\hline CSF1R-autoinhibited & CSF1R & 100 & 1000 \\
\hline CSK & CSK & 100 & 1000 \\
\hline CSNK1A1 & CSNK1A1 & 100 & 1000 \\
\hline CSNK1A1L & CSNK1A1L & 100 & 1000 \\
\hline CSNK1D & CSNK1D & 100 & 1000 \\
\hline CSNK1E & CSNK1E & 100 & 1000 \\
\hline CSNK1G1 & CSNK1G1 & 100 & 1000 \\
\hline CSNK1G2 & CSNK1G2 & 100 & 1000 \\
\hline CSNK1G3 & CSNK1G3 & 100 & 1000 \\
\hline CSNK2A1 & CSNK2A1 & 100 & 1000 \\
\hline CSNK2A2 & CSNK2A2 & 100 & 1000 \\
\hline CTK & MATK & 100 & 1000 \\
\hline DAPK1 & DAPK1 & 100 & 1000 \\
\hline DCAMKL2 & DCLK2 & 100 & 1000 \\
\hline DCAMKL3 & DCLK3 & 100 & 1000 \\
\hline DDR1 & DDR1 & 100 & 1000 \\
\hline DMPK2 & CDC42BPA & 100 & 1000 \\
\hline DRAK1 & STK17A & 100 & 1000 \\
\hline DRAK2 & STK17B & 100 & 1000 \\
\hline DYRK1B & DYRK1B & 100 & 1000 \\
\hline EGFR & EGFR & 100 & 1000 \\
\hline EGFR(G719S) & EGFR & 100 & 1000 \\
\hline EGFR(L747-E749del, A750P) & EGFR & 100 & 1000 \\
\hline EGFR(L747-S752del, P753S) & EGFR & 100 & 1000 \\
\hline EGFR(L858R) & EGFR & 100 & 1000 \\
\hline EGFR(T790M) & EGFR & 100 & 1000 \\
\hline EIF2AK1 & EIF2AK1 & 100 & 1000 \\
\hline EPHA1 & EPHA1 & 100 & 1000 \\
\hline EPHA2 & EPHA2 & 100 & 1000 \\
\hline EPHA3 & EPHA3 & 100 & 1000 \\
\hline EPHA5 & EPHA5 & 100 & 1000 \\
\hline EPHA6 & EPHAG & 100 & 1000 \\
\hline EPHA7 & EPHA7 & 100 & 1000 \\
\hline EPHA8 & EPHA8 & 100 & 1000 \\
\hline EPHB1 & EPHB1 & 100 & 1000 \\
\hline EPHB2 & EPHB2 & 100 & 1000 \\
\hline EPHB3 & EPHB3 & 100 & 1000 \\
\hline EPHB4 & EPHB4 & 100 & 1000 \\
\hline EPHB6 & EPHB6 & 100 & 1000 \\
\hline ERBB4 & ERBB4 & 100 & 1000 \\
\hline ERK1 & MAPK3 & 100 & 1000 \\
\hline ERK2 & MAPK1 & 100 & 1000 \\
\hline ERK3 & MAPK6 & 100 & 1000 \\
\hline
\end{tabular}




\begin{tabular}{|c|c|c|c|}
\hline CSNK1G3 & CSNK1G3 & 100 & 1000 \\
\hline CSNK2A1 & CSNK2A1 & 100 & 1000 \\
\hline CSNK2A2 & CSNK2A2 & 100 & 1000 \\
\hline CTK & MATK & 100 & 1000 \\
\hline DAPK1 & DAPK1 & 100 & 1000 \\
\hline DAPK2 & DAPK2 & 100 & 1000 \\
\hline DAPK3 & DAPK3 & 100 & 1000 \\
\hline DCAMKL2 & DCLK2 & 100 & 1000 \\
\hline DCAMKL3 & DCLK3 & 100 & 1000 \\
\hline DDR1 & DDR1 & 100 & 1000 \\
\hline DRAK1 & STK17A & 100 & 1000 \\
\hline DRAK2 & STK17B & 100 & 1000 \\
\hline DYRK2 & DYRK2 & 100 & 1000 \\
\hline EGFR & EGFR & 100 & 1000 \\
\hline EGFR(L747-S752del, P753S) & EGFR & 100 & 1000 \\
\hline EGFR(L858R) & EGFR & 100 & 1000 \\
\hline EPHA1 & EPHA1 & 100 & 1000 \\
\hline EPHA3 & EPHA3 & 100 & 1000 \\
\hline EPHA4 & EPHA4 & 100 & 1000 \\
\hline EPHA5 & EPHA5 & 100 & 1000 \\
\hline EPHA6 & EPHA6 & 100 & 1000 \\
\hline EPHA7 & EPHA7 & 100 & 1000 \\
\hline EPHA8 & EPHA8 & 100 & 1000 \\
\hline EPHB1 & EPHB1 & 100 & 1000 \\
\hline EPHB3 & EPHB3 & 100 & 1000 \\
\hline EPHB4 & EPHB4 & 100 & 1000 \\
\hline EPHB6 & EPHB6 & 100 & 1000 \\
\hline ERBB4 & ERBB4 & 100 & 1000 \\
\hline ERK1 & MAPK3 & 100 & 1000 \\
\hline ERK2 & MAPK1 & 100 & 1000 \\
\hline ERK3 & MAPK6 & 100 & 1000 \\
\hline ERK4 & MAPK4 & 100 & 1000 \\
\hline ERK5 & MAPK7 & 100 & 1000 \\
\hline ERK8 & MAPK15 & 100 & 1000 \\
\hline FER & FER & 100 & 1000 \\
\hline FES & FES & 100 & 1000 \\
\hline FGFR1 & FGFR1 & 100 & 1000 \\
\hline FGFR3(G697C) & FGFR3 & 100 & 1000 \\
\hline FGFR4 & FGFR4 & 100 & 1000 \\
\hline FGR & FGR & 100 & 1000 \\
\hline FLT3 & FLT3 & 100 & 1000 \\
\hline FLT3(D835H) & FLT3 & 100 & 1000 \\
\hline FLT4 & FLT4 & 100 & 1000 \\
\hline
\end{tabular}

\begin{tabular}{|c|c|c|c|}
\hline ERK4 & MAPK4 & 100 & 1000 \\
\hline ERK5 & MAPK7 & 100 & 1000 \\
\hline ERK8 & MAPK15 & 100 & 1000 \\
\hline ERN1 & ERN1 & 100 & 1000 \\
\hline FAK & PTK2 & 100 & 1000 \\
\hline FER & FER & 100 & 1000 \\
\hline FES & FES & 100 & 1000 \\
\hline FGFR1 & FGFR1 & 100 & 1000 \\
\hline FGFR2 & FGFR2 & 100 & 1000 \\
\hline FGFR3 & FGFR3 & 100 & 1000 \\
\hline FGFR3(G697C) & FGFR3 & 100 & 1000 \\
\hline FGFR4 & FGFR4 & 100 & 1000 \\
\hline FGR & FGR & 100 & 1000 \\
\hline FLT1 & FLT1 & 100 & 1000 \\
\hline FLT3 & FLT3 & 100 & 1000 \\
\hline FLT3(D835H) & FLT3 & 100 & 1000 \\
\hline FLT3(D835Y) & FLT3 & 100 & 1000 \\
\hline FLT3(ITD) & FLT3 & 100 & 1000 \\
\hline FLT3(K663Q) & FLT3 & 100 & 1000 \\
\hline FLT3(N841I) & FLT3 & 100 & 1000 \\
\hline FLT4 & FLT4 & 100 & 1000 \\
\hline FRK & FRK & 100 & 1000 \\
\hline GAK & GAK & 100 & 1000 \\
\hline GRK2 & ADRBK1 & 100 & 1000 \\
\hline GRK3 & ADRBK2 & 100 & 1000 \\
\hline GRK4 & GRK4 & 100 & 1000 \\
\hline GRK7 & GRK7 & 100 & 1000 \\
\hline GSK3A & GSK3A & 100 & 1000 \\
\hline $\mathrm{HCK}$ & HCK & 100 & 1000 \\
\hline HIPK1 & HIPK1 & 100 & 1000 \\
\hline HIPK2 & HIPK2 & 100 & 1000 \\
\hline HIPK3 & HIPK3 & 100 & 1000 \\
\hline HIPK4 & HIPK4 & 100 & 1000 \\
\hline HPK1 & MAP4K1 & 100 & 1000 \\
\hline HUNK & HUNK & 100 & 1000 \\
\hline IGF1R & IGF1R & 100 & 1000 \\
\hline IKK-epsilon & IKBKE & 100 & 1000 \\
\hline INSR & INSR & 100 & 1000 \\
\hline INSRR & INSRR & 100 & 1000 \\
\hline IRAK1 & IRAK1 & 100 & 1000 \\
\hline IRAK3 & IRAK3 & 100 & 1000 \\
\hline ITK & ITK & 100 & 1000 \\
\hline JAK1(JH1domain-catalytic) & JAK1 & 100 & 1000 \\
\hline
\end{tabular}




\begin{tabular}{|c|c|c|c|}
\hline FRK & FRK & 100 & 1000 \\
\hline GAK & GAK & 100 & 1000 \\
\hline GRK2 & ADRBK1 & 100 & 1000 \\
\hline GRK4 & GRK4 & 100 & 1000 \\
\hline GSK3A & GSK3A & 100 & 1000 \\
\hline HCK & $\mathrm{HCK}$ & 100 & 1000 \\
\hline HUNK & HUNK & 100 & 1000 \\
\hline IGF1R & IGF1R & 100 & 1000 \\
\hline IKK-epsilon & IKBKE & 100 & 1000 \\
\hline INSR & INSR & 100 & 1000 \\
\hline INSRR & INSRR & 100 & 1000 \\
\hline IRAK3 & IRAK3 & 100 & 1000 \\
\hline ITK & ITK & 100 & 1000 \\
\hline JAK1(JH1domain-catalytic) & JAK1 & 100 & 1000 \\
\hline JAK1(JH2domain-pseudokir & JJAK1 & 100 & 1000 \\
\hline JAK2(JH1domain-catalytic) & JAK2 & 100 & 1000 \\
\hline KIT(V559D,T670I) & KIT & 100 & 1000 \\
\hline KIT-autoinhibited & KIT & 100 & 1000 \\
\hline LATS1 & LATS1 & 100 & 1000 \\
\hline LATS2 & LATS2 & 100 & 1000 \\
\hline LIMK2 & LIMK2 & 100 & 1000 \\
\hline LKB1 & STK11 & 100 & 1000 \\
\hline LOK & STK10 & 100 & 1000 \\
\hline LRRK2(G2019S) & LRRK2 & 100 & 1000 \\
\hline LTK & LTK & 100 & 1000 \\
\hline MAР3КЗ & MAР3КЗ & 100 & 1000 \\
\hline MAP4K4 & MAP4K4 & 100 & 1000 \\
\hline MAP4K5 & MAP4K5 & 100 & 1000 \\
\hline MAPKAPK2 & MAPKAPK2 & 100 & 1000 \\
\hline MARK1 & MARK1 & 100 & 1000 \\
\hline MARK3 & MARK3 & 100 & 1000 \\
\hline MARK4 & MARK4 & 100 & 1000 \\
\hline MAST1 & MAST1 & 100 & 1000 \\
\hline MELK & MELK & 100 & 1000 \\
\hline MERTK & MERTK & 100 & 1000 \\
\hline MET & MET & 100 & 1000 \\
\hline MET(M1250T) & MET & 100 & 1000 \\
\hline $\operatorname{MET}(Y 1235 \mathrm{D})$ & MET & 100 & 1000 \\
\hline MKNK2 & MKNK2 & 100 & 1000 \\
\hline MLCK & MYLK3 & 100 & 1000 \\
\hline MLK1 & MAР3К9 & 100 & 1000 \\
\hline MLK3 & МАРЗК11 & 100 & 1000 \\
\hline MRCKA & CDC42BPA & 100 & 1000 \\
\hline
\end{tabular}

\begin{tabular}{|c|c|c|c|}
\hline JAK1(JH2domain-pseudokinas & JAK1 & 100 & 1000 \\
\hline JAK3(JH1domain-catalytic) & JAK3 & 100 & 1000 \\
\hline KIT & KIT & 100 & 1000 \\
\hline KIT(A829P) & KIT & 100 & 1000 \\
\hline KIT(D816V) & KIT & 100 & 1000 \\
\hline KIT(V559D,V654A) & KIT & 100 & 1000 \\
\hline KIT-autoinhibited & KIT & 100 & 1000 \\
\hline LATS1 & LATS1 & 100 & 1000 \\
\hline LATS2 & LATS2 & 100 & 1000 \\
\hline LCK & LCK & 100 & 1000 \\
\hline LIMK1 & LIMK1 & 100 & 1000 \\
\hline LIMK2 & LIMK2 & 100 & 1000 \\
\hline LKB1 & STK11 & 100 & 1000 \\
\hline LOK & STK10 & 100 & 1000 \\
\hline LRRK2(G2019S) & LRRK2 & 100 & 1000 \\
\hline LTK & LTK & 100 & 1000 \\
\hline LYN & LYN & 100 & 1000 \\
\hline LZK & MAP3K13 & 100 & 1000 \\
\hline MAР3К3 & МАРЗКЗ & 100 & 1000 \\
\hline MAP4K4 & MAP4K4 & 100 & 1000 \\
\hline MAP4K5 & MAP4K5 & 100 & 1000 \\
\hline MAPKAPK2 & MAPKAPK & 100 & 1000 \\
\hline MARK3 & MARK3 & 100 & 1000 \\
\hline MAST1 & MAST1 & 100 & 1000 \\
\hline MEK4 & MAP2K4 & 100 & 1000 \\
\hline MELK & MELK & 100 & 1000 \\
\hline MERTK & MERTK & 100 & 1000 \\
\hline MET & MET & 100 & 1000 \\
\hline MET(M1250T) & MET & 100 & 1000 \\
\hline MET(Y1235D) & MET & 100 & 1000 \\
\hline MKNK2 & MKNK2 & 100 & 1000 \\
\hline MLCK & MYLK3 & 100 & 1000 \\
\hline MLK1 & МАРЗК9 & 100 & 1000 \\
\hline MLK2 & MAР3К10 & 100 & 1000 \\
\hline MLK3 & MAP3K11 & 100 & 1000 \\
\hline MRCKA & CDC42BPA & 100 & 1000 \\
\hline MRCKB & CDC42BPB & 100 & 1000 \\
\hline MST1 & STK4 & 100 & 1000 \\
\hline MST1R & MST1R & 100 & 1000 \\
\hline MUSK & MUSK & 100 & 1000 \\
\hline MYLK & MYLK & 100 & 1000 \\
\hline MYLK2 & MYLK2 & 100 & 1000 \\
\hline MYO3A & MYO3A & 100 & 1000 \\
\hline
\end{tabular}




\begin{tabular}{|c|c|c|c|}
\hline MRCKB & CDC42BPB & 100 & 1000 \\
\hline MST1 & STK4 & 100 & 1000 \\
\hline MST1R & MST1R & 100 & 1000 \\
\hline MST3 & STK24 & 100 & 1000 \\
\hline MTOR & MTOR & 100 & 1000 \\
\hline MUSK & MUSK & 100 & 1000 \\
\hline MYLK4 & MYLK4 & 100 & 1000 \\
\hline MYOЗА & MYO3A & 100 & 1000 \\
\hline МYОзв & MYO3B & 100 & 1000 \\
\hline NEK1 & NEK1 & 100 & 1000 \\
\hline NEK10 & NEK10 & 100 & 1000 \\
\hline NEK2 & NEK2 & 100 & 1000 \\
\hline NEK3 & NEK3 & 100 & 1000 \\
\hline NEK4 & NEK4 & 100 & 1000 \\
\hline NEK6 & NEK6 & 100 & 1000 \\
\hline NEK7 & NEK7 & 100 & 1000 \\
\hline NEK9 & NEK9 & 100 & 1000 \\
\hline NLK & NLK & 100 & 1000 \\
\hline p38-alpha & MAPK14 & 100 & 1000 \\
\hline p38-beta & MAPK11 & 100 & 1000 \\
\hline p38-delta & MAPK13 & 100 & 1000 \\
\hline p38-gamma & MAPK12 & 100 & 1000 \\
\hline PAK1 & PAK1 & 100 & 1000 \\
\hline PAK3 & PAK3 & 100 & 1000 \\
\hline PAK4 & PAK4 & 100 & 1000 \\
\hline PAK6 & PAK6 & 100 & 1000 \\
\hline PAK7 & PAK7 & 100 & 1000 \\
\hline РСTK2 & CDK17 & 100 & 1000 \\
\hline РСTK3 & CDK18 & 100 & 1000 \\
\hline PDGFRA & PDGFRA & 100 & 1000 \\
\hline PDPK1 & PDPK1 & 100 & 1000 \\
\hline PFPK5(P.falciparum) & MAL13P1.279 & 100 & 1000 \\
\hline PFTAIRE2 & CDK15 & 100 & 1000 \\
\hline PFTK1 & CDK14 & 100 & 1000 \\
\hline PHKG1 & PHKG1 & 100 & 1000 \\
\hline PHKG2 & PHKG2 & 100 & 1000 \\
\hline PIK3C2G & PIK3C2G & 100 & 1000 \\
\hline PIK3CA & PIK3CA & 100 & 1000 \\
\hline PIK3СA(M1043I) & PIK3CA & 100 & 1000 \\
\hline PIK3CA(Q546K) & РIКЗСА & 100 & 1000 \\
\hline PIK3СB & РІКЗСВ & 100 & 1000 \\
\hline PIK3CG & PIK3CG & 100 & 1000 \\
\hline PIKFYVE & PIKFYVE & 100 & 1000 \\
\hline
\end{tabular}

\begin{tabular}{|c|c|c|c|}
\hline MYO3B & MYOзB & 100 & 1000 \\
\hline NEK1 & NEK1 & 100 & 1000 \\
\hline NEK11 & NEK11 & 100 & 1000 \\
\hline NEK2 & NEK2 & 100 & 1000 \\
\hline NEK3 & NEK3 & 100 & 1000 \\
\hline NEK4 & NEK4 & 100 & 1000 \\
\hline NEK5 & NEK5 & 100 & 1000 \\
\hline NEK6 & NEK6 & 100 & 1000 \\
\hline NEK7 & NEK7 & 100 & 1000 \\
\hline NEK9 & NEK9 & 100 & 1000 \\
\hline NIK & MAP3K14 & 100 & 1000 \\
\hline NLK & NLK & 100 & 1000 \\
\hline p38-alpha & MAPK14 & 100 & 1000 \\
\hline p38-beta & MAPK11 & 100 & 1000 \\
\hline p38-delta & MAPK13 & 100 & 1000 \\
\hline p38-gamma & MAPK12 & 100 & 1000 \\
\hline PAK3 & PAK3 & 100 & 1000 \\
\hline PAK4 & PAK4 & 100 & 1000 \\
\hline PAK6 & PAK6 & 100 & 1000 \\
\hline PCTK1 & CDK16 & 100 & 1000 \\
\hline PCTK2 & CDK17 & 100 & 1000 \\
\hline PCTK3 & CDK18 & 100 & 1000 \\
\hline PDGFRA & PDGFRA & 100 & 1000 \\
\hline PDPK1 & PDPK1 & 100 & 1000 \\
\hline PFPK5(P.falciparum) & MAL13P1. & 100 & 1000 \\
\hline PFTK1 & CDK14 & 100 & 1000 \\
\hline PHKG1 & PHKG1 & 100 & 1000 \\
\hline PIK3С2B & PIK3C2B & 100 & 1000 \\
\hline PIK3C2G & PIK3C2G & 100 & 1000 \\
\hline PIK3CA & PIK3CA & 100 & 1000 \\
\hline PIK3CA(C420R) & PIK3CA & 100 & 1000 \\
\hline PIK3CA(H1047L) & PIK3CA & 100 & 1000 \\
\hline PIK3CA(M1043I) & PIKЗСА & 100 & 1000 \\
\hline РІКЗСВ & PIKЗСВ & 100 & 1000 \\
\hline PIK3CG & PIK3CG & 100 & 1000 \\
\hline PIM1 & PIM1 & 100 & 1000 \\
\hline PIM3 & PIM3 & 100 & 1000 \\
\hline PIP5K2B & PIP4K2B & 100 & 1000 \\
\hline PKAC-alpha & PRKACA & 100 & 1000 \\
\hline PKAC-beta & PRKACB & 100 & 1000 \\
\hline PKN1 & PKN1 & 100 & 1000 \\
\hline PKN2 & PKN2 & 100 & 1000 \\
\hline PKNB(M.tuberculosis) & pknB & 100 & 1000 \\
\hline
\end{tabular}




\begin{tabular}{|c|c|c|c|}
\hline PIP5K1A & PIP5K1A & 100 & 1000 \\
\hline PIP5K2B & PIP4K2B & 100 & 1000 \\
\hline PIP5K2C & PIP4K2C & 100 & 1000 \\
\hline PKAC-beta & PRKACB & 100 & 1000 \\
\hline PKN2 & PKN2 & 100 & 1000 \\
\hline PKNB(M.tuberculosis) & pknB & 100 & 1000 \\
\hline PLK1 & PLK1 & 100 & 1000 \\
\hline PLK4 & PLK4 & 100 & 1000 \\
\hline PRKCD & PRKCD & 100 & 1000 \\
\hline PRKCH & PRKCH & 100 & 1000 \\
\hline PRKCQ & PRKCQ & 100 & 1000 \\
\hline PRKD1 & PRKD1 & 100 & 1000 \\
\hline PRKG1 & PRKG1 & 100 & 1000 \\
\hline PRKR & EIF2AK2 & 100 & 1000 \\
\hline PRKX & PRKX & 100 & 1000 \\
\hline PRP4 & PRPF4B & 100 & 1000 \\
\hline PYK2 & PTK2B & 100 & 1000 \\
\hline RAF1 & RAF1 & 100 & 1000 \\
\hline RET & RET & 100 & 1000 \\
\hline RET(M918T) & RET & 100 & 1000 \\
\hline RET(V804L) & RET & 100 & 1000 \\
\hline $\operatorname{RET}(\mathrm{V} 804 \mathrm{M})$ & RET & 100 & 1000 \\
\hline RIPK1 & RIPK1 & 100 & 1000 \\
\hline RIPK2 & RIPK2 & 100 & 1000 \\
\hline RPS6KA4(Kin.Dom.1-N-term & RPS6KA4 & 100 & 1000 \\
\hline RPS6KA5(Kin.Dom.1-N-term & RPS6KA5 & 100 & 1000 \\
\hline RSK1(Kin.Dom.2-C-terminal & RPS6KA1 & 100 & 1000 \\
\hline RSK2(Kin.Dom.1-N-termina & RPS6KA3 & 100 & 1000 \\
\hline RSK3(Kin.Dom.1-N-termina & RPS6KA2 & 100 & 1000 \\
\hline RSK3(Kin.Dom.2-C-terminal & RPS6KA2 & 100 & 1000 \\
\hline RSK4(Kin.Dom.1-N-termina & RPS6KA6 & 100 & 1000 \\
\hline RSK4(Kin.Dom.2-C-terminal & RPS6KA6 & 100 & 1000 \\
\hline SgK110 & SgK110 & 100 & 1000 \\
\hline SRPK3 & SRPK3 & 100 & 1000 \\
\hline STK16 & STK16 & 100 & 1000 \\
\hline STK35 & STK35 & 100 & 1000 \\
\hline STK36 & STK36 & 100 & 1000 \\
\hline STK39 & STK39 & 100 & 1000 \\
\hline TEC & TEC & 100 & 1000 \\
\hline TESK1 & TESK1 & 100 & 1000 \\
\hline TGFBR1 & TGFBR1 & 100 & 1000 \\
\hline TGFBR2 & TGFBR2 & 100 & 1000 \\
\hline TIE1 & TIE1 & 100 & 1000 \\
\hline
\end{tabular}

\begin{tabular}{|c|c|c|c|}
\hline PLK1 & PLK1 & 100 & 1000 \\
\hline PLK4 & PLK4 & 100 & 1000 \\
\hline PRKCD & PRKCD & 100 & 1000 \\
\hline PRKCH & PRKCH & 100 & 1000 \\
\hline PRKCl & PRKCI & 100 & 1000 \\
\hline PRKCQ & PRKCQ & 100 & 1000 \\
\hline PRKD1 & PRKD1 & 100 & 1000 \\
\hline PRKG1 & PRKG1 & 100 & 1000 \\
\hline PRKG2 & PRKG2 & 100 & 1000 \\
\hline PRKX & PRKX & 100 & 1000 \\
\hline PRP4 & PRPF4B & 100 & 1000 \\
\hline PYK2 & PTK2B & 100 & 1000 \\
\hline RET & RET & 100 & 1000 \\
\hline RET(M918T) & RET & 100 & 1000 \\
\hline RET(V804L) & RET & 100 & 1000 \\
\hline RET(V804M) & RET & 100 & 1000 \\
\hline RIOK2 & RIOK2 & 100 & 1000 \\
\hline RIOK3 & RIOK3 & 100 & 1000 \\
\hline ROCK2 & ROCK2 & 100 & 1000 \\
\hline ROS1 & ROS1 & 100 & 1000 \\
\hline RPS6KA4(Kin.Dom.1-N-termin & RPS6KA4 & 100 & 1000 \\
\hline RPS6KA5(Kin.Dom.1-N-termin & RPS6KA5 & 100 & 1000 \\
\hline RSK3(Kin.Dom.1-N-terminal) & RPS6KA2 & 100 & 1000 \\
\hline SgK110 & SgK110 & 100 & 1000 \\
\hline SGK2 & SGK2 & 100 & 1000 \\
\hline SIK & SIK1 & 100 & 1000 \\
\hline SIK2 & SIK2 & 100 & 1000 \\
\hline SLK & SLK & 100 & 1000 \\
\hline SRC & SRC & 100 & 1000 \\
\hline SRPK1 & SRPK1 & 100 & 1000 \\
\hline SRPK2 & SRPK2 & 100 & 1000 \\
\hline SRPK3 & SRPK3 & 100 & 1000 \\
\hline STK16 & STK16 & 100 & 1000 \\
\hline STK35 & STK35 & 100 & 1000 \\
\hline STK36 & STK36 & 100 & 1000 \\
\hline STK39 & STK39 & 100 & 1000 \\
\hline TEC & TEC & 100 & 1000 \\
\hline TESK1 & TESK1 & 100 & 1000 \\
\hline TGFBR2 & TGFBR2 & 100 & 1000 \\
\hline TIE1 & TIE1 & 100 & 1000 \\
\hline TIE2 & TEK & 100 & 1000 \\
\hline TLK2 & TLK2 & 100 & 1000 \\
\hline TNK2 & TNK2 & 100 & 1000 \\
\hline
\end{tabular}




\begin{tabular}{|l|l|r|r|}
\hline TIE2 & TEK & 100 & 1000 \\
\hline TLK1 & TLK1 & 100 & 1000 \\
\hline TLK2 & TLK2 & 100 & 1000 \\
\hline TNK2 & TNK2 & 100 & 1000 \\
\hline TRKA & NTRK1 & 100 & 1000 \\
\hline TRKB & NTRK2 & 100 & 1000 \\
\hline TSSK3 & TSSK3 & 100 & 1000 \\
\hline WEE1 & WEE1 & 100 & 1000 \\
\hline WEE2 & WEE2 & 100 & 1000 \\
\hline WNK3 & WNK3 & 100 & 1000 \\
\hline YANK2 & STK32B & 100 & 1000 \\
\hline YANK3 & STK32C & 100 & 1000 \\
\hline YES & YES1 & 100 & 1000 \\
\hline YSK1 & STK25 & 100 & 1000 \\
\hline ZAK & ZAK & 100 & 1000 \\
\hline
\end{tabular}

\begin{tabular}{|l|l|r|r|}
\hline TRKC & NTRK3 & 100 & 1000 \\
\hline TSSK3 & TSSK3 & 100 & 1000 \\
\hline TTK & TTK & 100 & 1000 \\
\hline TXK & TXK & 100 & 1000 \\
\hline TYK2(JH1domain-catalytic) & TYK2 & 100 & 1000 \\
\hline ULK2 & ULK2 & 100 & 1000 \\
\hline VEGFR2 & KDR & 100 & 1000 \\
\hline VPS34 & PIK3C3 & 100 & 1000 \\
\hline WNK1 & WNK1 & 100 & 1000 \\
\hline WNK3 & WNK3 & 100 & 1000 \\
\hline WNK4 & WNK4 & 100 & 1000 \\
\hline YANK1 & STK32A & 100 & 1000 \\
\hline YES & YES1 & 100 & 1000 \\
\hline YSK1 & STK25 & 100 & 1000 \\
\hline ZAK & ZAK & 100 & 1000 \\
\hline
\end{tabular}


Kinases inhibited $<50 \%$ annotated with $\mathbf{4 6 d}$ shown in black circles and $47 \mathrm{~b}$ shown in blue triangles

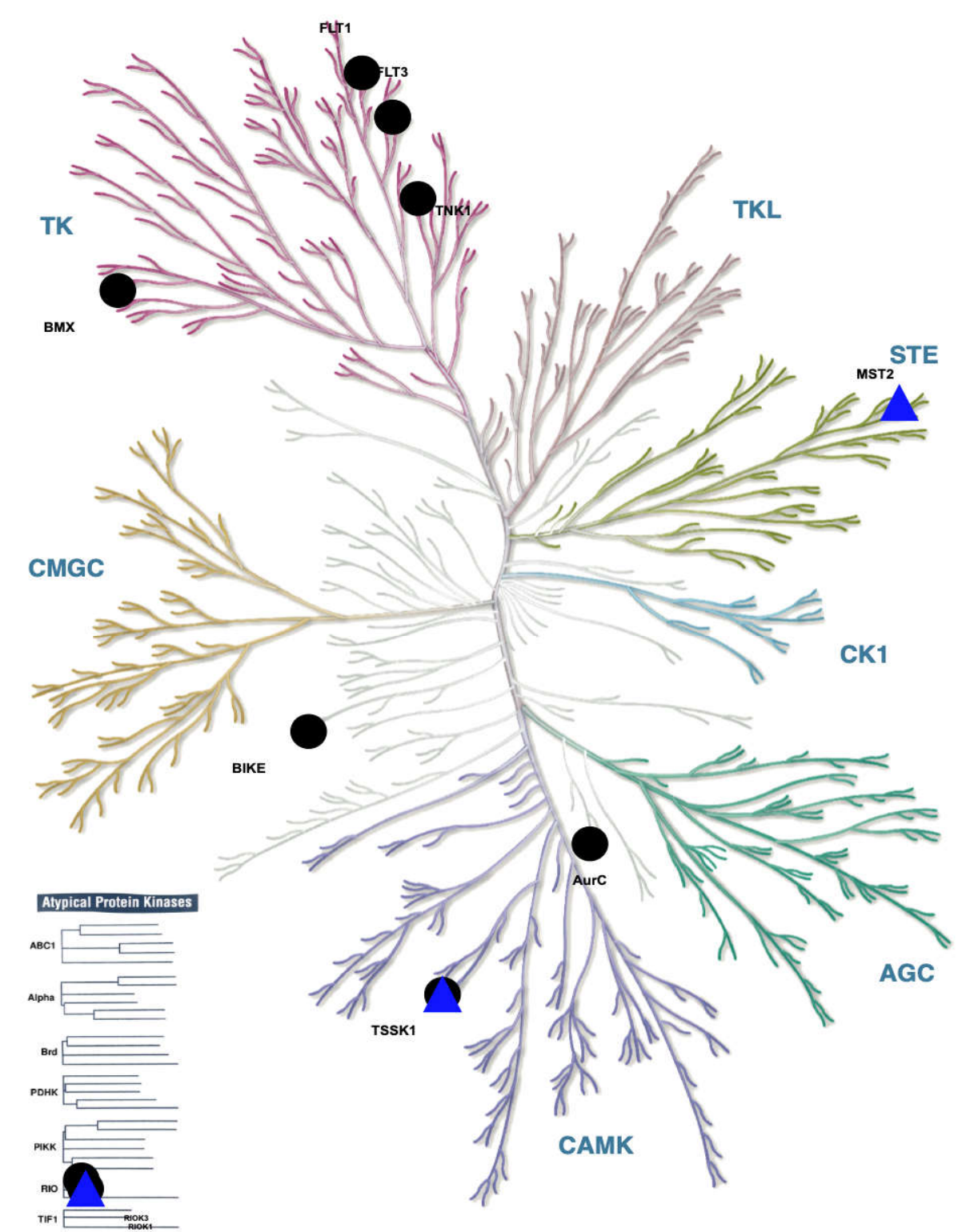




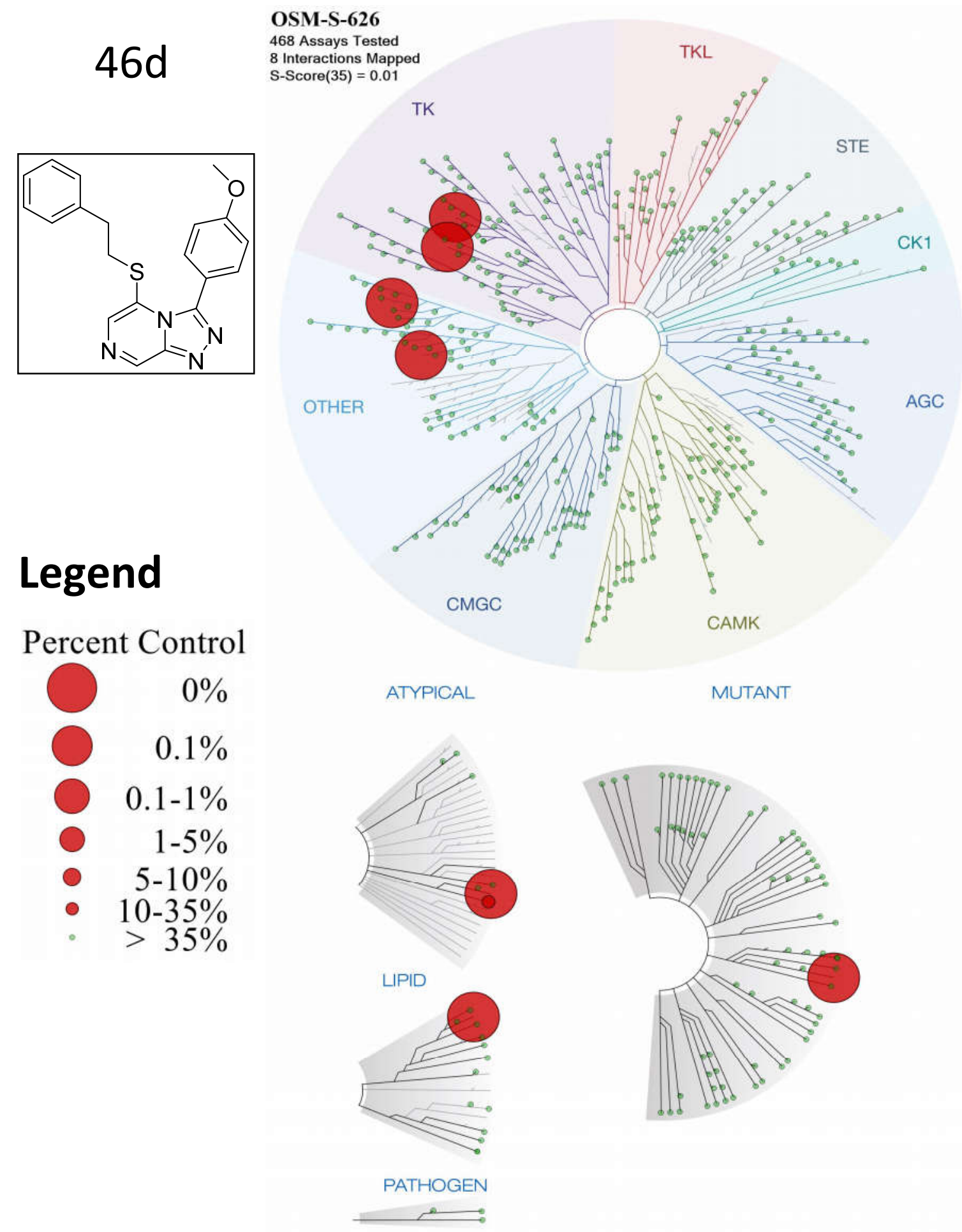




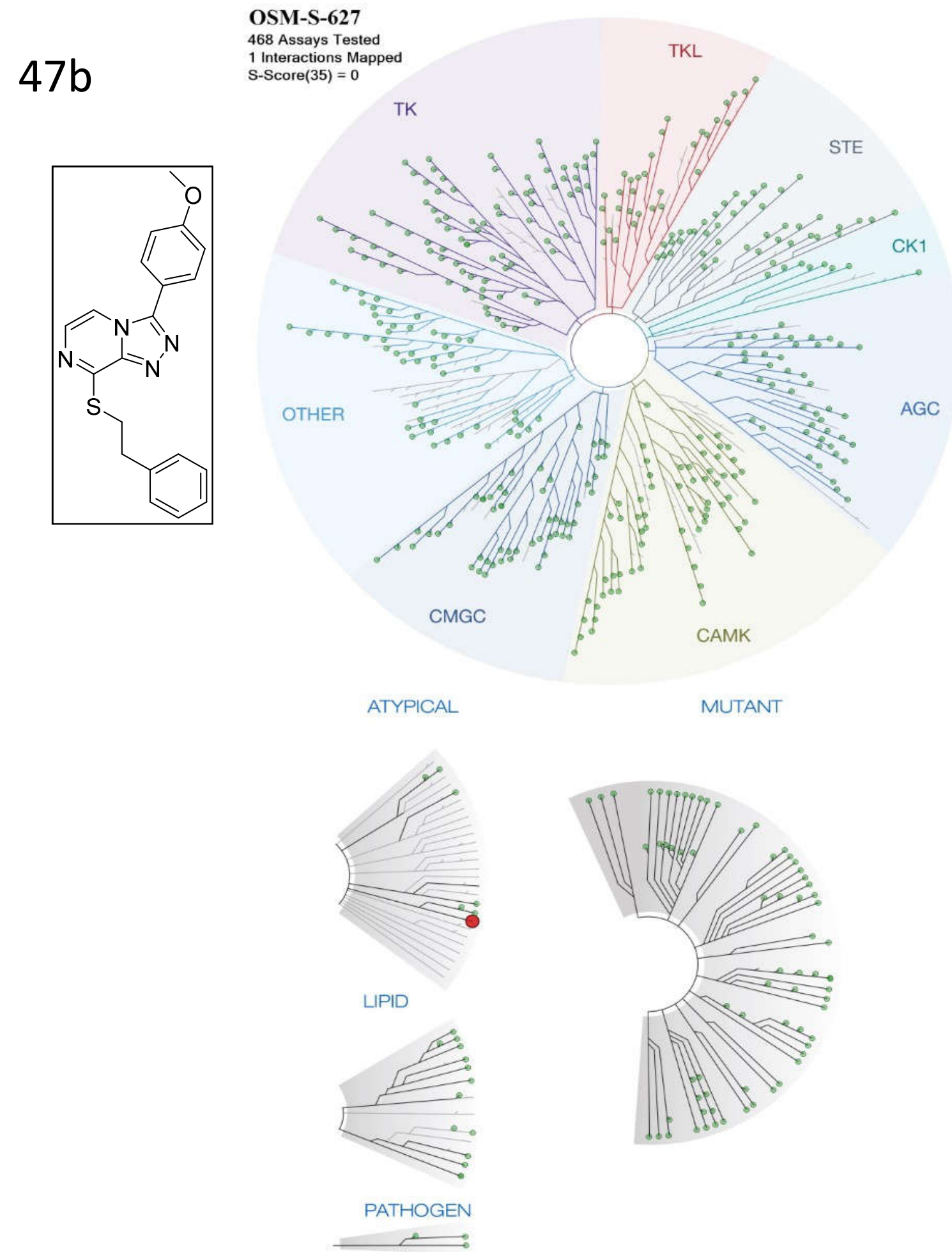


Other files

X-ray data.zip (2.53 MiB)

view on ChemRxiv • download file 